Алгебра и анализ

Том. 17 (2005), вып. 6
St. Petersburg Math. J.

Vol. 17 (2006), No. 6, Pages 897-973

S 1061-0022(06)00935-6

Article electronically published on September 21, 2006

\title{
HOMOGENIZATION WITH CORRECTOR TERM FOR PERIODIC ELLIPTIC DIFFERENTIAL OPERATORS
}

\author{
M. SH. BIRMAN AND T. A. SUSLINA
}

\begin{abstract}
We continue to study the class of matrix periodic elliptic differential operators $\mathcal{A}_{\varepsilon}$ in $\mathbb{R}^{d}$ with coefficients oscillating rapidly (i.e., depending on $\mathbf{x} / \varepsilon$ ). This class was introduced in the authors' earlier work of 2001 and 2003. The problem of homogenization in the small period limit is considered. Approximation for the resolvent $\left(\mathcal{A}_{\varepsilon}+I\right)^{-1}$ is obtained in the operator norm in $L_{2}\left(\mathbb{R}^{d}\right)$ with error term of order $\varepsilon^{2}$. The so-called corrector is taken into account. We develop the approach of our paper of 2003, where approximation with no corrector term but with remainder term of order $\varepsilon$ was found. The paper is based on the operator-theoretic material obtained in our paper in the previous issue of this journal. Though the present paper is a continuation of the earlier work, it can be read independently.
\end{abstract}

\section{Contents}

Introduction

Chapter 1. Preliminaries

$\S 1$. Operator-theory method

$\S 2$. Periodic differential operators in $L_{2}\left(\mathbb{R}^{d} ; \mathbb{C}^{n}\right)$. Direct integral expansion

$\S 3$. The effective matrix and the effective operator

Chapter 2. Threshold approximations for resolvents of differential operators

$\S 4$. Approximation of the resolvent $\left(\widehat{\mathcal{A}}(\mathbf{k})+\varepsilon^{2} I\right)^{-1}$

$\S 5$. Approximation of the generalized resolvent $\left(\widehat{\mathcal{A}}(\mathbf{k})+\varepsilon^{2} Q\right)^{-1}$

$\S 6$. Approximation of the resolvent $\left(\widehat{\mathcal{A}}+\varepsilon^{2} I\right)^{-1}$

$\S 7$. Approximation of the generalized resolvent $\left(\widehat{\mathcal{A}}+\varepsilon^{2} Q\right)^{-1}$

Chapter 3. Homogenization with corrector term for periodic differential operators

$\S 8$. Approximation of the resolvent $\left(\widehat{\mathcal{A}}_{\varepsilon}+I\right)^{-1}$

9. Approximation for $\left(\widehat{\mathcal{A}}_{\varepsilon}+Q^{\varepsilon}\right)^{-1}$ and $\left(\mathcal{A}_{\varepsilon}+I\right)^{-1}$

Chapter 4. Applications of the general theory

$\S 10$. The operator $\widehat{\mathcal{A}}=\mathbf{D}^{*} g \mathbf{D}$

$\S 11$. The periodic Schrödinger operator

$\S 12$. The two-dimensional periodic Pauli operator

$\S 13$. The operator of elasticity theory

$\S 14$. The model operator of electrodynamics

$\S 15$. Comments and references

References

2000 Mathematics Subject Classification. Primary 35J99.

Key words and phrases. Periodic operators, threshold approximations, homogenization, corrector. Supported by RFBR (grant nos. 05-01-01076-a and 05-01-02944-YaF-a). 


\section{INTRODUCTION}

0.1. In the present paper, the authors' approach to homogenization theory (see BSu1, $\mathrm{BSu} 2$ ) is developed. In [BSu1, BSu2, sharp-order estimates for the difference of the resolvents of a periodic differential operator (DO) with rapidly oscillating coefficients and of the homogenized DO with constant coefficients were obtained for the first time. Under our approach, the mathematical nature of the homogenization procedure was elucidated neatly as a threshold effect near the bottom of the spectrum of a periodic DO. As a result, new machinery of investigation for a wide class of linear homogenization problems was created. We dealt (and continue to deal here) with second-order periodic matrix DOs of the form

$$
\mathcal{A}=\mathcal{A}(g, f)=f(\mathbf{x})^{*} b(\mathbf{D})^{*} g(\mathbf{x}) b(\mathbf{D}) f(\mathbf{x})
$$

acting in $L_{2}\left(\mathbb{R}^{d} ; \mathbb{C}^{n}\right)$. To avoid repetition, we refer the reader to Subsection 2.1. Now we only mention the following: a DO of the form (0.1) is an elliptic second-order operator admitting factorization. Its coefficients $f$ and $g$ are matrix-valued functions periodic with respect to some lattice $\Gamma$ in $\mathbb{R}^{d} ; f$ and $g$ are not assumed to be smooth. Significant attention is paid to the class of operators of the form

$$
\widehat{\mathcal{A}}=\widehat{\mathcal{A}}(g)=b(\mathbf{D})^{*} g(\mathbf{x}) b(\mathbf{D}) .
$$

This class is narrower than (0.1). The operators (0.1) and (0.2) are viewed as selfadjoint operators in $L_{2}\left(\mathbb{R}^{d} ; \mathbb{C}^{n}\right)$. The bottom of the spectrum of the operator $\mathcal{A}(g, f)$ is the point $\lambda=0$ :

$$
\min \operatorname{spec} \mathcal{A}(g, f)=0 .
$$

Along with the operators (0.1) and (0.2), we consider operators with rapidly oscillating coefficients:

$$
\begin{aligned}
\mathcal{A}_{\varepsilon}(g, f) & =f\left(\varepsilon^{-1} \mathbf{x}\right)^{*} b(\mathbf{D})^{*} g\left(\varepsilon^{-1} \mathbf{x}\right) b(\mathbf{D}) f\left(\varepsilon^{-1} \mathbf{x}\right), & & \varepsilon>0, \\
\widehat{\mathcal{A}}_{\varepsilon}(g) & =b(\mathbf{D})^{*} g\left(\varepsilon^{-1} \mathbf{x}\right) b(\mathbf{D}), & & \varepsilon>0 .
\end{aligned}
$$

The homogenization problem consists in the study of the asymptotic behavior of these operators as $\varepsilon \rightarrow 0$. Restricting ourselves to the case of the operator (0.5) for simplicity, we consider the equation

$$
\widehat{\mathcal{A}}_{\varepsilon}(g) \mathbf{u}_{\varepsilon}+\mathbf{u}_{\varepsilon}=\mathbf{F}, \quad \mathbf{F} \in L_{2}\left(\mathbb{R}^{d} ; \mathbb{C}^{n}\right) .
$$

There exists a DO (the homogenized or effective operator)

$$
\widehat{\mathcal{A}}^{0}:=\widehat{\mathcal{A}}\left(g^{0}\right)=b(\mathbf{D})^{*} g^{0} b(\mathbf{D})
$$

with constant matrix $g^{0}$ such that, in a sense,

$$
\begin{gathered}
\mathbf{u}_{\varepsilon} \longrightarrow \mathbf{u}_{0}, \\
\varepsilon \longrightarrow 0 .
\end{gathered}
$$

Here $\mathbf{u}_{0}$ is the solution of the homogenized equation

$$
\widehat{\mathcal{A}}^{0} \mathbf{u}_{0}+\mathbf{u}_{0}=\mathbf{F} \text {. }
$$

The matrix $g^{0}$ is determined by a well-known procedure that requires solving an auxiliary boundary value problem on the cell of the lattice $\Gamma$; this periodic boundary value problem does not involve the parameter $\varepsilon$. Next, the way of understanding the limit in (0.8) (together with due conditions on $g$ and $\mathbf{F}$ ) must be clarified, and also, if possible, the error term should be estimated. For the operators $(0.4)$ the problem is formulated in a similar but more complicated way. 
In BSu1, BSu2, it was established for the first time that (0.8) can be understood in the following sense:

$$
\left\|\left(\widehat{\mathcal{A}}_{\varepsilon}+I\right)^{-1}-\left(\widehat{\mathcal{A}}^{0}+I\right)^{-1}\right\|_{L_{2}\left(\mathbb{R}^{d} ; \mathbb{C}^{n}\right) \rightarrow L_{2}\left(\mathbb{R}^{d} ; \mathbb{C}^{n}\right)} \leq c \varepsilon,
$$

where the constant $c$ depends only on the lattice $\Gamma$ and on the upper and lower bounds for the matrix $g$ and for the symbol $b(\boldsymbol{\theta})^{*} b(\boldsymbol{\theta})$ (see Subsection 2.1 below).

0.2. Estimate (0.10) was proved (see [BSu1, BSu2]) in three steps. A large portion of the work was done within an abstract operator theory approach. With the help of perturbation theory, an appropriate finite rank approximation was found for the resolvent $\left(A(t)+\varepsilon^{2} I\right)^{-1}$. Here $A(t)$ is a family of selfadjoint operators admitting factorization and quadratic with respect to the parameter $t \in \mathbb{R}$ (for the details about the family $A(t)$, see Subsection 1.1). The second step was based on partial diagonalization of the operator $(0.2)$ via the unitary Gelfand transformation. As a result, the study of the resolvent $\left(\widehat{\mathcal{A}}(g)+\varepsilon^{2} I\right)^{-1}$ was reduced to the study of the resolvent $\left(\widehat{A}(t)+\varepsilon^{2} I\right)^{-1}$ for some operator family $\widehat{A}(t)$ fitting into a certain abstract pattern. The threshold nature of the problem lies in the fact that the investigation boils down to the study of the resolvent at the point $-\varepsilon^{2}$, i.e., near the bottom of the spectrum $\lambda=0$ (see (0.3)). As a result, after the inverse Gelfand transformation, we arrive at the estimate

$$
\varepsilon\left\|\left(\widehat{\mathcal{A}}(g)+\varepsilon^{2} I\right)^{-1}-\left(\widehat{\mathcal{A}}^{0}+\varepsilon^{2} I\right)^{-1}\right\|_{L_{2}\left(\mathbb{R}^{d} ; \mathbb{C}^{n}\right) \rightarrow L_{2}\left(\mathbb{R}^{d} ; \mathbb{C}^{n}\right)} \leq c, \quad \varepsilon>0 .
$$

Finally, the third step is a simple passage from (0.11) to (0.10) by a (unitary) scaling transformation.

We emphasize that the plan described above turns out to be realistic because the scaling transformation can easily be taken into account in the operator norm estimates. (However, this transformation presents a serious obstruction in the study of convergence of various types.)

A similar plan was applied to the study of homogenization problems for the more general operators (0.4). However, in this case the answer is not so simple. In order to obtain the corresponding result, preliminarily we need to study the "generalized resolvent" for the operators (0.5), i.e., to investigate the behavior of the operator-valued function

$$
\left(\widehat{\mathcal{A}}_{\varepsilon}(g)+Q\left(\varepsilon^{-1} \mathbf{x}\right)\right)^{-1}
$$

as $\varepsilon \rightarrow 0$. Here $Q(\mathbf{x})$ is a uniformly positive definite and bounded Hermitian matrixvalued function.

0.3. The corrector. The further goal of homogenization theory is to find a more accurate approximation for the solution $\mathbf{u}_{\varepsilon}$ of equation (0.6) by adding appropriate terms to the solution $\mathbf{u}_{0}$ of equation (0.9). First of all, a term of order $\varepsilon$, the so-called corrector, is introduced. The expression for the "traditional" corrector (at least for some particular cases of the operators (0.5)) is well known. Usually, it is assumed that the corresponding matrix-valued function $g$ and the vector-valued function $\mathbf{F}$ are sufficiently smooth.

In the present paper, we also introduce a corrector, but without any additional smoothness assumptions. At this point, we discuss only operators (0.5), though the more general operators (0.4) are also studied in the paper. The problem is to find a relatively simple operator $K(\varepsilon)$ such that

$$
\left\|\left(\widehat{\mathcal{A}}_{\varepsilon}(g)+I\right)^{-1}-\left(\widehat{\mathcal{A}}^{0}+I\right)^{-1}-\varepsilon K(\varepsilon)\right\|_{L_{2}\left(\mathbb{R}^{d} ; \mathbb{C}^{n}\right) \rightarrow L_{2}\left(\mathbb{R}^{d} ; \mathbb{C}^{n}\right)} \leq c_{1} \varepsilon^{2}, \quad \varepsilon>0 .
$$

Thus, again, we want to approximate the resolvent $\left(\widehat{\mathcal{A}}_{\varepsilon}(g)+I\right)^{-1}$ in the operator norm. Herewith, the operator $K(\varepsilon)$ is bounded in the operator norm uniformly in $\varepsilon$, and the constant $c_{1}$ depends only on the same parameters as the constant $c$ in (0.10). We have succeeded in obtaining such an estimate. However, we were forced to add two new terms 
to the "traditional" corrector. One of them is the operator adjoint to the traditional corrector. Another term (the operator $K_{3}$ in our notation) is more essential, since it does not depend on $\varepsilon$. For the authors, the appearance of the operator $K_{3}$ was somewhat unexpected. We pay special attention to the analysis of this term. In particular, we distinguish the cases where $K_{3}=0$ and where, in general, $K_{3} \neq 0$. Note that the full corrector $K(\varepsilon)$ itself is defined only up to $O(\varepsilon)$. But the term $K_{3}$ is defined in a unique way, being independent of $\varepsilon$. More details about the operators $K(\varepsilon)$ and $K_{3}$ can be found in $\S 8$, especially in Subsections 8.5 and 8.6, and also in Subsection 15.1.

To further improve the error estimate, terms of higher order can be added to the approximation of the resolvent $\left(\widehat{\mathcal{A}}_{\varepsilon}(g)+I\right)^{-1}$. However, this cannot be done without additional restrictions on the matrix-valued function $g$.

0.4. The plan of exposition. To a great extent, the plan copies the structure of the papers BSu1, BSu2. Here we can distinguish three steps similar to those described in Subsection 0.2. The difference is that now at each step we must take the corrector into account. Clearly, the method becomes bulkier. This stimulated the authors to separate the first part, namely, the abstract method, in the paper [BSu5]. However, all necessary preliminaries from that paper and also from $\mathrm{BSu} 2$ are given below in Chapter 1. Thus, the present text can be read independently of those papers.

Chapter 2 is devoted to the second step, namely, to obtaining threshold approximations for the resolvents. The main technical material of the paper is collected in that chapter. Chapter 3 corresponds to the third (and final) step and contains the final results of the paper. They concern the homogenization problems themselves. Finally, the extensive Chapter 4 is devoted to examples and applications of the general results of Chapter 3 . On one hand, these applications are important in themselves. On the other hand, they "test" the general theorems. The choice of applications is motivated by the desire to illustrate various effects hidden in the general method.

For more detailed information about the structure and content of the paper, we refer the reader to the table of contents and to the abstracts at the beginning of each chapter.

The paper contains full proofs of all statements. However, it can be viewed as a survey because, together with [BSu2, BSu5], it gives a clear exposition of the new approach to homogenization theory developed by the authors and elucidates the nature of the results obtained in this way. Furthermore, some comments of a survey character are contained in the final $\S 15$.

We try to formulate all the statements so that they can be read independently. Explicit expressions for constants in the estimates are traced throughout. Often we distinguish various particular and special cases where general results become simpler. We realize that, as a result of all this, the text is somewhat heavy. At the same time, the character of the exposition must show that the "calculus" constructed in the paper allows one to go far in the analysis of specific situations.

In order to understand the essence and technique of our method, it suffices to consider the case of the operators $(0.2),(0.5)$. This case corresponds to $\S 1$ (Subsections 1.1-1.5), $\S 2, \S 3$ (Subsections 3.1-3.3), $\$ 4, \S 6, \S 8$, and also to part of Chapter 4.

0.5. For the first time, estimates of the form (0.10) appeared in the papers BSu1, BSu2]. Estimates of the form (0.13) in two particular cases were pointed out in [BSu4. The papers [Su1]- Su4 were also based on the method developed by the authors. In Su3, Su4, the "elliptic" technique was carried over to parabolic problems. The papers Su1, Su2] were devoted to the stationary periodic Maxwell operator. We dwell on this operator in more detail. 
First of all, the Maxwell operator is not semibounded and even not elliptic. However, if we restrict this operator to the divergence free subspaces (which is natural), then it becomes elliptic. Next, by some technical tricks, the study of this operator can be reduced to the analysis of some positive second-order DOs. However, in general, the latter operators do not belong to the class of operators (0.1). More detailed comments on the Maxwell operator can be found in Subsection 14.2. Here we only note that, prior to the papers [BSu2, Chapter 7] and [Su1, Su2], in the homogenization problem for the Maxwell operator only analogs of relation $(0.8)$, understood in the sense of weak $L_{2}\left(\mathbb{R}^{3} ; \mathbb{C}^{6}\right)$-convergence, had been known. However, the "elliptic" rule for the construction of effective characteristics of the Maxwell operator (see (14.5)) was well known.

In homogenization theory, the papers BSu1, BSu2 stimulated interest in the error estimates for approximations in the operator norms. We mention the very recent papers by V. V. Zhikov with coauthors and also papers by G. Griso. $\S 15$ contains more details about these papers and other publications on homogenization theory.

0.6. Notation. Let $\mathcal{G}$ and $\mathcal{G}_{*}$ be separable Hilbert spaces. The symbols $(\cdot, \cdot)_{\mathcal{G}}$ and $\|\cdot\|_{\mathcal{G}}$ denote the inner product and the norm in $\mathcal{G}$; the symbol $\|\cdot\|_{\mathcal{G} \rightarrow \mathcal{G}_{*}}$ stands for the norm of a continuous linear operator from $\mathcal{G}$ to $\mathcal{G}_{*}$. Sometimes, we omit the indices if this does not lead to confusion. By $I=I_{\mathcal{G}}$ we denote the identity operator in $\mathcal{G}$, and $\mathbb{O}=\mathbb{O}_{\mathcal{G}}$ is the null operator. If $A: \mathcal{G} \rightarrow \mathcal{G}_{*}$ is a linear operator, then $\operatorname{Dom} A$ is the domain of $A$. If $\mathfrak{N}$ is a subspace in $\mathcal{G}$, then $\mathfrak{N}^{\perp}:=\mathcal{G} \ominus \mathfrak{N}$. If $P$ is the orthogonal projection of $\mathcal{G}$ onto $\mathfrak{N}$, then $P^{\perp}$ denotes the orthogonal projection onto $\mathfrak{N}^{\perp}$. The spectrum of a closed operator $T$ in $\mathcal{G}$ is denoted by $\operatorname{spec} T$. The symbol $\langle\cdot, \cdot\rangle=\langle\cdot, \cdot\rangle_{n}$ stands for the standard inner product in $\mathbb{C}^{n},|\cdot|$ denotes the norm of a vector in $\mathbb{C}^{n}$; the unit $(n \times n)$-matrix is denoted by $\mathbf{1}=\mathbf{1}_{n}$. For $z \in \mathbb{C}$, we denote by $z^{*}$ the complex conjugate number; this nonstandard notation is used because the notation $\bar{g}$ is reserved for the mean value of a matrix-valued function $g$ (see (3.8) below). For an $(m \times n)$-matrix $a$, the symbol $|a|$ stands for the norm of this matrix viewed as a linear operator from $\mathbb{C}^{n}$ to $\mathbb{C}^{m} ; a^{t}$ is the transpose, and $a^{*}$ is the adjoint $(n \times m)$-matrix.

The $L_{p}$-classes of $\mathbb{C}^{n}$-valued functions defined on a domain $\mathcal{O} \subset \mathbb{R}^{d}$ are denoted by $L_{p}\left(\mathcal{O} ; \mathbb{C}^{n}\right), 1 \leq p \leq \infty$. The Sobolev classes of order $s$ with integrability index $p$ of $\mathbb{C}^{n}$-valued functions (on a domain $\mathcal{O} \subseteq \mathbb{R}^{d}$ ) are denoted by $W_{p}^{s}\left(\mathcal{O} ; \mathbb{C}^{n}\right)$. For $p=2$, we denote this space by $H^{s}\left(\mathcal{O} ; \mathbb{C}^{n}\right), s \in \mathbb{R}$. If $n=1$, we write simply $L_{p}(\mathcal{O}), W_{p}^{s}(\mathcal{O}), H^{s}(\mathcal{O})$, etc. Sometimes (if this does not lead to confusion), we use abbreviated notation also for spaces of vector-valued or matrix-valued functions.

Next, $\mathbf{x}=\left(x^{1}, \ldots, x^{d}\right) \in \mathbb{R}^{d}, i D_{j}=\partial_{j}=\partial / \partial x^{j}, j=1, \ldots, d, \nabla=\operatorname{grad}=$ $\left(\partial_{1}, \ldots, \partial_{d}\right), \mathbf{D}=-i \nabla=\left(D_{1}, \ldots, D_{d}\right)$

By $C, c, \mathcal{C}, \mathfrak{C}$ (possibly, with indices and marks) we denote various constants in the estimates.

0.7. Acknowledgements. We are grateful to all colleagues who showed their interest in our work on homogenization theory. Their opinion was important for us, because we came to homogenization theory from the outside and somewhat unexpectedly for ourselves. Especially, we would like to thank V. V. Zhikov, S. A. Nazarov, and A. L. Pyatnitsky for detailed and fruitful discussions.

The opportunity to work in the scientific institutions of Sweden was of great importance for us. The final versions of a number of our papers on homogenization were written there. We mention with gratitude the hospitality and support of the Royal Institute of Technology (Stockholm), the Mittag-Leffler Institute (Dujrsholm), and the Mathematics Department of the Chalmers University of Technology (Göteborg). Especially, we are grateful to professors A. Laptev, K.-O. Widman and G. Rozenblum. 


\section{Chapter 1. Preliminaries}

Our main goal in this chapter is to make it possible to read the paper independently of the earlier papers [BSu1, BSu2, BSu5]. The present paper is a continuation of those papers and is based on them. Therefore, here we give the material necessary for what follows and taken mainly from [BSu2, BSu5, where full proofs can be found, and we try to keep the notation. Some details in this chapter are considered for the first time; in such cases we present the proofs.

$\S 1$ contains material of an abstract nature. In $\S 2$, we recall some facts about the class of periodic differential operators introduced in BSu1, BSu2 and studied also in the present paper. In $\S 3$ we collect facts about the "effective characteristics" for the operator class mentioned above. All our results on homogenization are formulated in terms of these characteristics.

The material of this chapter is referred to in subsequent sections repeatedly.

\section{$\S 1$. OpERATOR-THEORY METHOD}

In this section, we briefly describe the abstract fundamentals of the operator (spectral) approach to homogenization problems for periodic differential operators. We follow the earlier publications [BSu2, BSu5], where one can find full proofs. In this sense, Subsections 1.6 and 1.7 are exceptional: their content was published before only in part.

Our main attention is paid to formulations of the results from BSu5 on threshold approximations with corrector term for the resolvent of a factorized operator family.

1.1. Factorized families. Let $\mathfrak{H}$ and $\mathfrak{H}_{*}$ be two complex separable Hilbert spaces. Suppose $X_{0}: \mathfrak{H} \rightarrow \mathfrak{H}_{*}$ is a densely defined and closed operator and $X_{1}: \mathfrak{H} \rightarrow \mathfrak{H}_{*}$ is a bounded operator. On the domain $\operatorname{Dom} X(t)=\operatorname{Dom} X_{0}$, we introduce the operator $X(t):=X_{0}+t X_{1}, t \in \mathbb{R}$. Our main object is the family of selfadjoint positive operators

$$
A(t):=X(t)^{*} X(t), \quad t \in \mathbb{R}
$$

in $\mathfrak{H}$. The operator $(1.1)$ is generated in $\mathfrak{H}$ by the closed quadratic form $\|X(t) u\|_{\mathfrak{H}_{*}}^{2}$, $u \in \operatorname{Dom} X_{0}$. We put $A(0)=X_{0}^{*} X_{0}=: A_{0}$ and

$$
\mathfrak{N}:=\operatorname{Ker} A_{0}=\operatorname{Ker} X_{0}, \quad \mathfrak{N}_{*}:=\operatorname{Ker} X_{0}^{*} .
$$

Suppose that the point $\lambda_{0}=0$ is an isolated point of the spectrum of $A_{0}$ and that

$$
0<n:=\operatorname{dim} \mathfrak{N}<\infty, \quad n \leq n_{*}:=\operatorname{dim} \mathfrak{N}_{*} \leq \infty
$$

We denote by $d^{0}$ the distance from the point $\lambda_{0}=0$ to the rest of the spectrum of $A_{0}$. Let $F(t, s)$ be the spectral projection of the operator $A(t)$ corresponding to the interval $[0, s]$. We fix a number $\delta>0$ such that $8 \delta<d^{0}$ and put

$$
t^{0}=t^{0}(\delta)=\delta^{1 / 2}\left\|X_{1}\right\|^{-1}
$$

where $\left\|X_{1}\right\|=\left\|X_{1}\right\|_{\mathfrak{H} \rightarrow \mathfrak{H}_{*}}$. It turns out (see $\left.\left.\mathrm{BSu} 2,(1.1 .3)\right]\right)$ that $F(t, \delta)=F(t, 3 \delta)$ and $\operatorname{rank} F(t, \delta)=n$ for $|t| \leq t^{0}$. Let $P$ be the orthogonal projection of $\mathfrak{H}$ onto $\mathfrak{N}$, and let $P_{*}$ be the orthogonal projection of $\mathfrak{H}_{*}$ onto $\mathfrak{N}_{*}$.

1.2. The operators $Z$ and $R$. For $\omega \in \mathfrak{N}$, let $\psi=\psi(\omega) \in \operatorname{Dom} X_{0} \cap \mathfrak{N}^{\perp}$ be a (weak) solution of the equation

$$
X_{0}^{*}\left(X_{0} \psi+X_{1} \omega\right)=0
$$

We define a bounded operator $Z: \mathfrak{H} \rightarrow \mathfrak{H}$ by the relation

$$
Z u=\psi(P u), \quad u \in \mathfrak{H}
$$


(see [BSu5, Subsection 1.2]). Clearly,

$$
\operatorname{rank} Z \leq n, \quad Z \mathfrak{N} \subset \mathfrak{N}^{\perp}, \quad Z \mathfrak{N}^{\perp}=0 .
$$

The operator $Z$ satisfies the estimate

$$
\|Z\|_{\mathfrak{H} \rightarrow \mathfrak{H}} \leq(8 \delta)^{-1 / 2}\left\|X_{1}\right\|
$$

(see [BSu5, (1.8)]).

Now we define a linear operator $R: \mathfrak{N} \rightarrow \mathfrak{N}_{*}$ (see [BSu2, Subsection 1.1.2]) by the relation $R \omega=X_{0} \psi(\omega)+X_{1} \omega$. Another description of $R$ is given by

$$
R=\left.P_{*} X_{1}\right|_{\mathfrak{N}} .
$$

1.3. The spectral germ. The selfadjoint operator

$$
S=R^{*} R: \mathfrak{N} \rightarrow \mathfrak{N}
$$

is called the spectral germ of the operator family (1.1) at $t=0$ (see BSu2, Subsection 1.1.3]). The spectral germ can also be written as

$$
S=\left.P X_{1}^{*} P_{*} X_{1}\right|_{\mathfrak{N}} .
$$

We say that the germ $S$ is nondegenerate if $\operatorname{Ker} S=\{0\}$.

Let $\widetilde{A}(t)=\widetilde{X}(t)^{*} \widetilde{X}(t)$ be yet another operator family in $\mathfrak{H}$ having the form (1.1) and subject to the same conditions as $A(t)$ (here $\widetilde{\mathfrak{H}}_{*}$ and $\mathfrak{H}_{*}$ may be different). All the objects related to $\widetilde{A}(t)$ are marked by " $\sim$ ". The families $A(t)$ and $\widetilde{A}(t)$ are said to be threshold equivalent if $\mathfrak{N}=\widetilde{\mathfrak{N}}$ and $S=\widetilde{S}$.

In accordance with the general analytic perturbation theory for selfadjoint operators (see $\left[\mathrm{Ka}\right.$ ), for $|t| \leq t^{0}$ there exist real-analytic functions $\lambda_{l}(t)$ and real-analytic $\mathfrak{H}$-valued functions $\varphi_{l}(t)$ such that

$$
A(t) \varphi_{l}(t)=\lambda_{l}(t) \varphi_{l}(t), \quad l=1, \ldots, n,|t| \leq t^{0}=t^{0}(\delta),
$$

and these functions $\varphi_{l}(t), l=1, \ldots, n$, form an orthonormal basis in the subspace $F(t, \delta) \mathfrak{H}$. For some sufficiently small $t_{*} \leq t^{0}$, we have convergent power series expansions

$$
\begin{aligned}
& \lambda_{l}(t)=\gamma_{l} t^{2}+\mu_{l} t^{3}+\cdots, \quad \gamma_{l} \geq 0, \quad \mu_{l} \in \mathbb{R}, \quad l=1, \ldots, n, \quad|t| \leq t_{*}, \\
& \varphi_{l}(t)=\omega_{l}+t \varphi_{l}^{(1)}+t^{2} \varphi_{l}^{(2)}+\cdots, \quad l=1, \ldots, n, \quad|t| \leq t_{*} .
\end{aligned}
$$

The elements $\omega_{l}=\varphi_{l}(0), l=1, \ldots, n$, form an orthonormal basis in $\mathfrak{N}$. Substituting (1.7) and (1.8) in (1.6) and comparing the coefficients of $t$ and $t^{2}$, we arrive at the formulas (see [BSu5, (1.15), (1.16)])

$$
\begin{aligned}
\widetilde{\omega}_{l}:=\varphi_{l}^{(1)}-\psi\left(\omega_{l}\right)=\varphi_{l}^{(1)}-Z \omega_{l} \in \mathfrak{N}, & l & =1, \ldots, n, \\
S \omega_{l}=\gamma_{l} \omega_{l}, & l & =1, \ldots, n
\end{aligned}
$$

(see BSu5, (1.15), (1.16)]).

Relations (1.10) clarify the spectral meaning of the germ $S$ and imply that

$$
S P=\sum_{l=1}^{n} \gamma_{l}\left(\cdot, \omega_{l}\right)_{\mathfrak{H}} \omega_{l} .
$$

From (1.11) it is clear that the germ $S$ does not depend on the choice of a factorization of the form (1.1) for $A(t)$.

We note that

$$
\left(\widetilde{\omega}_{k}, \omega_{l}\right)_{\mathfrak{H}}+\left(\omega_{k}, \widetilde{\omega}_{l}\right)_{\mathfrak{H}}=0, \quad k, l=1, \ldots, n
$$

(see [BSu5, (1.18)]). 
1.4. The operator $N$. We shall need the following bounded selfadjoint operator in $\mathfrak{H}$ (see [BSu5, (4.13)]):

$$
N=Z^{*} X_{1}^{*} R P+(R P)^{*} X_{1} Z
$$

Observe that

$$
N \mathfrak{N} \subset \mathfrak{N}, \quad N \mathfrak{N}^{\perp}=\mathbb{O} .
$$

We have

$$
\|N\|_{\mathfrak{H} \rightarrow \mathfrak{H}} \leq(2 \delta)^{-1 / 2}\left\|X_{1}\right\|^{3}
$$

(see BSu5, (4.16)]). The operator $N$ can also be represented as a sum (see BSu5, Subsection 4.2]):

$$
N=N_{0}+N_{*},
$$

where

$$
\begin{aligned}
& N_{0}=\sum_{l=1}^{n} \mu_{l}\left(\cdot, \omega_{l}\right)_{\mathfrak{H}} \omega_{l}, \\
& N_{*}=\sum_{l=1}^{n} \gamma_{l}\left(\left(\cdot, \widetilde{\omega}_{l}\right)_{\mathfrak{H}} \omega_{l}+\left(\cdot, \omega_{l}\right)_{\mathfrak{H}} \widetilde{\omega}_{l}\right) .
\end{aligned}
$$

Here $\gamma_{l}, \mu_{l}, \omega_{l}$, and $\widetilde{\omega}_{l}$ are defined by (1.7)-(1.9).

We give expressions for the matrix entries of the operators $N_{0}$ and $N_{*}$ in the basis $\omega_{1}, \ldots, \omega_{n}$. Obviously,

$$
\left(N_{0} \omega_{j}, \omega_{k}\right)_{\mathfrak{H}}=\delta_{j k} \mu_{k}, \quad j, k=1, \ldots, n,
$$

i.e., the matrix of $N_{0}$ is diagonal. Next,

$$
\left(N_{*} \omega_{j}, \omega_{k}\right)_{\mathfrak{H}}=\gamma_{k}\left(\omega_{j}, \widetilde{\omega}_{k}\right)_{\mathfrak{H}}+\gamma_{j}\left(\widetilde{\omega}_{j}, \omega_{k}\right)_{\mathfrak{H}}, \quad j, k=1, \ldots, n,
$$

and, by (1.12),

$$
\left(N_{*} \omega_{k}, \omega_{k}\right)_{\mathfrak{H}}=0, \quad k=1, \ldots, n,
$$

i.e., the matrix of $N_{*}$ has zero diagonal. In particular, if $n=1$, then $N_{*}=0$. From (1.15), (1.18), and (1.19) it is seen that

$$
\left(N \omega_{l}, \omega_{l}\right)_{\mathfrak{H}}=\mu_{l}, \quad l=1, \ldots, n .
$$

1.5. Approximation of the resolvent $\left(A(t)+\varepsilon^{2} I\right)^{-1}$ for small $\varepsilon$. Assume that, for some $c_{*}>0$, we have

$$
A(t) \geq c_{*} t^{2} I, \quad|t| \leq t^{0} .
$$

The following result was proved in [BSu5, Theorem 5.5].

Theorem 1.1. Suppose that the families $A(t)$ and $\widetilde{A}(t)$ are threshold equivalent, condition (1.21) is fulfilled, and $\widetilde{A}(t)$ satisfies a similar condition with constant $\widetilde{c}_{*}$. Moreover, let $\widetilde{Z}=0$ (where $\widetilde{Z}$ is the analog of the operator $Z$ for the family $\widetilde{A}(t)$ ). Then, for

$$
|t| \leq t_{\min }^{0}=\min \left\{t^{0}, \widetilde{t}^{0}\right\}
$$

and $\varepsilon>0$, we have

$$
\begin{aligned}
\left(A(t)+\varepsilon^{2} I\right)^{-1}= & \left(\widetilde{A}(t)+\varepsilon^{2} I\right)^{-1}+t\left(Z\left(\widetilde{A}(t)+\varepsilon^{2} I\right)^{-1}+\left(\widetilde{A}(t)+\varepsilon^{2} I\right)^{-1} Z^{*}\right) \\
& -t^{3}\left(\widetilde{A}(t)+\varepsilon^{2} I\right)^{-1} N\left(\widetilde{A}(t)+\varepsilon^{2} I\right)^{-1}+\mathcal{J}_{*} .
\end{aligned}
$$


Here the operator $Z$ is defined by (1.3) and (1.4), and the operator $N$ is defined by (1.13). The remainder term $\mathcal{J}_{*}$ in (1.23) satisfies the following estimate uniform in $\varepsilon$ and $t$ :

$$
\left\|\mathcal{J}_{*}\right\|_{\mathfrak{H} \rightarrow \mathfrak{H}} \leq C_{*}=C+\widetilde{C}\left(1+2^{-1 / 2}+2^{-1 / 2}\left(c_{*}^{-1}+\widetilde{c}_{*}^{-1}\right)\left\|X_{1}\right\|^{2}\right),
$$

where

$$
\begin{aligned}
& C=\delta^{-1}\left(\beta_{1} c_{*}^{-1}\left\|X_{1}\right\|^{2}+\beta_{2} c_{*}^{-2}\left\|X_{1}\right\|^{4}+\beta_{3} c_{*}^{-3}\left\|X_{1}\right\|^{6}+3^{-1}\right), \\
& \widetilde{C}=\widetilde{\delta}^{-1}\left(\beta_{1} \widetilde{c}_{*}^{-1}\left\|\widetilde{X}_{1}\right\|^{2}+\beta_{2} \widetilde{c}_{*}^{-2}\left\|\widetilde{X}_{1}\right\|^{4}+\beta_{3} \widetilde{c}_{*}^{-3}\left\|\widetilde{X}_{1}\right\|^{6}+3^{-1}\right),
\end{aligned}
$$

and the $\beta_{j}, j=1,2,3$, are absolute constants.

It was the presence of terms with $t$ and $t^{3}$ in (1.23) that allowed us to obtain the uniform estimate (1.24) for the "remainder term". These terms are of the same order $s^{-1}$ with respect to the parameter $s=\left(t^{2}+\varepsilon^{2}\right)^{1 / 2}$, while the principal term $\left(\widetilde{A}(t)+\varepsilon^{2} I\right)^{-1}$ is of order $s^{-2}$. Without taking these terms into account, the norm of the difference $\left(A(t)+\varepsilon^{2} I\right)^{-1}-\left(\widetilde{A}(t)+\varepsilon^{2} I\right)^{-1}$ can be estimated only by $C \varepsilon^{-1}$ (see Theorem 1.5.6 in BSu2]). Taking these terms into consideration corresponds to including the corrector in homogenization problems.

If $Z=0$ (and then also $N=0$ ), we obtain the following result (see BSu5, Theorem $5.4])$.

Theorem 1.2. Suppose that the families $A(t)$ and $\widetilde{A}(t)$ are threshold equivalent and satisfy conditions of the form (1.21). Suppose also that $Z=0$ and $\widetilde{Z}=0$. Then, under condition (1.22), we have

$$
\left\|\left(A(t)+\varepsilon^{2} I\right)^{-1}-\left(\widetilde{A}(t)+\varepsilon^{2} I\right)^{-1}\right\|_{\mathfrak{H} \rightarrow \mathfrak{H}} \leq C+\widetilde{C}, \quad \varepsilon>0,
$$

where the constants $C$ and $\widetilde{C}$ are defined by (1.25) and (1.26).

1.6. The family of the form $A(t)=M^{*} \widehat{A}(t) M$. Along with the space $\mathfrak{H}$, we consider yet another Hilbert space $\widehat{\mathfrak{H}}$ and the family $\widehat{X}(t)=\widehat{X}_{0}+t \widehat{X}_{1}: \widehat{\mathfrak{H}} \rightarrow \mathfrak{H}_{*}$ of the same form as $X(t)$. Here, the space $\mathfrak{H}_{*}$ remains unchanged. Let $M: \mathfrak{H} \rightarrow \widehat{\mathfrak{H}}$ be an isomorphism; suppose that $M \operatorname{Dom} X_{0}=\operatorname{Dom} \widehat{X}_{0}, X(t)=\widehat{X}(t) M, X_{0}=\widehat{X}_{0} M, X_{1}=\widehat{X}_{1} M$. In $\widehat{\mathfrak{H}}$, we consider the family of selfadjoint operators

$$
\widehat{A}(t)=\widehat{X}(t)^{*} \widehat{X}(t)
$$

Then

$$
A(t)=M^{*} \widehat{A}(t) M .
$$

Below, we mark all the objects corresponding to the family $\widehat{A}(t)$ by "^". Observe (see [BSu2, Subsection 1.1.5]) that $\widehat{\mathfrak{N}}=M \mathfrak{N}, \widehat{\mathfrak{N}}_{*}=\mathfrak{N}_{*}, R=\left.\widehat{R} M\right|_{\mathfrak{N}}$ and

$$
S=\left.P M^{*} \widehat{S} M\right|_{\mathfrak{N}} \text {. }
$$

We introduce the notation

$$
Q:=\left(M M^{*}\right)^{-1}=\left(M^{*}\right)^{-1} M^{-1}: \widehat{\mathfrak{H}} \rightarrow \widehat{\mathfrak{H}} .
$$

The operator $Q$ is positive, and both $Q$ and $Q^{-1}$ are continuous. If the family $\widehat{A}(t)$ and the positive definite operator $Q$ in $\widehat{\mathfrak{H}}$ are given initially, then the operator $Q$ admits a (nonunique) representation of the form (1.30). Then the family $A(t)$ can be recovered by formula (1.28). 
Let $Q_{\widehat{\mathfrak{N}}}$ be the block of the operator $Q$ in the subspace $\widehat{\mathfrak{N}}$, i.e., $Q_{\widehat{\mathfrak{N}}}=\left.\widehat{P} Q\right|_{\widehat{\mathfrak{N}}}: \widehat{\mathfrak{N}} \rightarrow \widehat{\mathfrak{N}}$. The orthogonal projection $P$ of $\mathfrak{H}$ onto $\mathfrak{N}$ and the orthogonal projection $\widehat{P}$ of $\widehat{\mathfrak{H}}$ onto $\widehat{\mathfrak{N}}$ satisfy the relation

$$
P=M^{-1}\left(Q_{\widehat{\mathfrak{N}}}\right)^{-1} \widehat{P}\left(M^{*}\right)^{-1}
$$

(see [Su3, Proposition 1.2]). By (1.29) and (1.31), we have

$$
S=\left.M^{-1}\left(Q_{\widehat{\mathfrak{N}}}\right)^{-1} \widehat{S} M\right|_{\mathfrak{N}}
$$

Now, we describe the numbers $\gamma_{l}$ and the elements $\omega_{l}$ occurring in (1.7) and (1.8) in terms of the operators $\widehat{S}$ and $Q_{\widehat{\mathfrak{N}}}$. We put $\widehat{\phi}_{l}:=M \omega_{l} \in \widehat{\mathfrak{N}}, l=1, \ldots, n$. Then (1.10) and (1.32) imply that

$$
\widehat{S} \widehat{\phi}_{l}=\gamma_{l} Q_{\widehat{\mathfrak{N}}} \widehat{\phi}_{l}, \quad \widehat{\phi}_{l}=M \omega_{l}, \quad l=1, \ldots, n .
$$

Since $\left(\omega_{j}, \omega_{k}\right)_{\mathfrak{H}}=\left(Q_{\widehat{\mathfrak{N}}} \widehat{\phi}_{j}, \widehat{\phi_{k}}\right)_{\widehat{\mathfrak{H}}}$, the elements $\widehat{\phi}_{1}, \ldots, \widehat{\phi}_{n}$ form a basis in $\widehat{\mathfrak{N}}$, which is orthonormal with weight $Q_{\widehat{\mathfrak{N}}}$ :

$$
\left(Q_{\widehat{\mathfrak{N}}} \widehat{\phi}_{j}, \widehat{\phi_{k}}\right)_{\widehat{\mathfrak{H}}}=\delta_{j k}, \quad j, k=1, \ldots, n .
$$

1.7. The operators $\widehat{Z}_{Q}$ and $\widehat{N}_{Q}$. We put $\widehat{Z}_{Q}=M Z M^{-1} \widehat{P}$. By BSu5, Lemma 6.1], $\widehat{Z}_{Q}$ is the operator in $\widehat{\mathfrak{H}}$ that takes an element $\widehat{u} \in \widehat{\mathfrak{H}}$ to a (unique) solution $\widehat{\psi}_{Q} \in \widehat{\mathfrak{H}}$ of the equation

$$
\widehat{X}_{0}^{*}\left(\widehat{X}_{0} \widehat{\psi}_{Q}+\widehat{X}_{1} \widehat{\omega}\right)=0, \quad Q \widehat{\psi}_{Q} \perp \widehat{\mathfrak{N}}
$$

where $\widehat{\omega}=\widehat{P} \widehat{u} \in \widehat{\mathfrak{N}}$. We put

$$
\widehat{N}_{Q}:=\widehat{P}\left(M^{*}\right)^{-1} N M^{-1} \widehat{P} .
$$

The operator $\widehat{N}_{Q}$ also admits the following representation (see [BSu5, Lemma 6.2]):

$$
\widehat{N}_{Q}=\widehat{Z}_{Q}^{*} \widehat{X}_{1}^{*} \widehat{R} \widehat{P}+(\widehat{R} \widehat{P})^{*} \widehat{X}_{1} \widehat{Z}_{Q} .
$$

The operators $\widehat{Z}_{Q}$ and $\widehat{N}_{Q}$ satisfy the estimates

$$
\begin{aligned}
&\left\|\widehat{Z}_{Q}\right\|_{\widehat{\mathfrak{H}} \rightarrow \widehat{\mathfrak{H}}} \leq(8 \delta)^{-1 / 2}\left\|X_{1}\right\|\|M\|\left\|M^{-1}\right\|, \\
&\left\|\widehat{N}_{Q}\right\|_{\widehat{\mathfrak{H}} \rightarrow \widehat{\mathfrak{H}}} \leq(2 \delta)^{-1 / 2}\left\|X_{1}\right\|\|M\|\left\|M^{-1}\right\|\left\|\widehat{X}_{1}\right\|^{2}
\end{aligned}
$$

(see [BSu5, (6.17), (6.20)]).

Formulas (1.15) and (1.36) show that the operator $\widehat{N}_{Q}$ can be represented as

$$
\widehat{N}_{Q}=\widehat{N}_{0, Q}+\widehat{N}_{*, Q} \text {, }
$$

where $\widehat{N}_{0, Q}:=\widehat{P}\left(M^{*}\right)^{-1} N_{0} M^{-1} \widehat{P}, \widehat{N}_{*, Q}:=\widehat{P}\left(M^{*}\right)^{-1} N_{*} M^{-1} \widehat{P}$. Putting $\widehat{\phi}_{l}=M \omega_{l} \in \widehat{\mathfrak{N}}$ and $\widetilde{\psi}_{l}=M \widetilde{\omega}_{l} \in \widehat{\mathfrak{N}}$ and using (1.16) and (1.17), we obtain

$$
\begin{aligned}
& \widehat{N}_{0, Q}=\sum_{l=1}^{n} \mu_{l}\left(\cdot, Q_{\widehat{\mathfrak{N}}} \widehat{\phi}_{l}\right)_{\widehat{\mathfrak{H}}} Q_{\widehat{\mathfrak{N}}} \widehat{\phi}_{l}, \\
& \widehat{N}_{*, Q}=\sum_{l=1}^{n} \gamma_{l}\left(\left(\cdot, Q_{\widehat{\mathfrak{N}}} \widetilde{\psi}_{l}\right)_{\widehat{\mathfrak{H}}} Q_{\widehat{\mathfrak{N}}} \widehat{\phi}_{l}+\left(\cdot, Q_{\widehat{\mathfrak{N}}} \widehat{\phi}_{l}\right)_{\widehat{\mathfrak{H}}} Q_{\widehat{\mathfrak{N}}} \widetilde{\psi}_{l}\right) .
\end{aligned}
$$

From (1.34), (1.41), and (1.42) it follows that

$$
\begin{array}{ll}
\left(\widehat{N}_{0, Q} \widehat{\phi}_{j}, \widehat{\phi}_{k}\right)_{\widehat{\mathfrak{H}}}=\mu_{j} \delta_{j k}, & j, k=1, \ldots, n, \\
\left(\widehat{N}_{*, Q} \widehat{\phi}_{j}, \widehat{\phi}_{k}\right)_{\widehat{\mathfrak{H}}}=\gamma_{k}\left(\widehat{\phi}_{j}, Q_{\widehat{\mathfrak{N}}} \widetilde{\psi}_{k}\right)_{\widehat{\mathfrak{H}}}+\gamma_{j}\left(Q_{\widehat{\mathfrak{N}}} \widetilde{\psi}_{j}, \widehat{\phi}_{k}\right)_{\widehat{\mathfrak{H}}}, & j, k=1, \ldots, n .
\end{array}
$$


Note that relations (1.12) imply the following identities:

$$
\left(Q_{\widehat{\mathfrak{N}}} \widetilde{\psi}_{k}, \widehat{\phi}_{l}\right)_{\widehat{\mathfrak{H}}}+\left(\widehat{\phi}_{k}, Q_{\widehat{\mathfrak{N}}} \widetilde{\psi}_{l}\right)_{\widehat{\mathfrak{H}}}=0, \quad k, l=1, \ldots, n .
$$

By (1.44) and (1.45), we have

$$
\left(\widehat{N}_{*, Q} \widehat{\phi}_{j}, \widehat{\phi}_{j}\right)_{\widehat{H}}=0, \quad j=1, \ldots, n .
$$

Relations (1.40), (1.43), and (1.46) lead to the following identities:

$$
\left(\widehat{N}_{Q} \widehat{\phi}_{l}, \widehat{\phi}_{l}\right)_{\widehat{\mathfrak{H}}}=\mu_{l}, \quad l=1, \ldots, n .
$$

1.8. Approximation of the generalized resolvent. The selfadjoint operator

$$
\left(\widehat{A}(t)+\varepsilon^{2} Q\right)^{-1}, \quad \varepsilon>0,
$$

in $\widehat{\mathfrak{H}}$ is called the generalized resolvent (or $Q$-resolvent) for the family $\widehat{A}(t)$. Relations (1.28) and (1.30) imply that

$$
\left(\widehat{A}(t)+\varepsilon^{2} Q\right)^{-1}=M\left(A(t)+\varepsilon^{2} I\right)^{-1} M^{*} .
$$

Now, let $\widehat{A}_{+}(t)$ be yet another family of the form (1.27) acting in $\widehat{\mathfrak{H}}$, and let $Q_{+}$be a positive definite operator in $\widehat{\mathfrak{H}}$. We put $M_{+}=\left(Q_{+}\right)^{-1 / 2}$, and introduce the operator family $A_{+}(t)=M_{+} \widehat{A}_{+}(t) M_{+}$. Suppose that $A_{+}(t)$ satisfies the analog of condition (1.21) with constant $c_{*}^{(+)}$for $|t| \leq t_{+}^{0}$. Then $\widehat{A}_{+}(t)$ automatically satisfies a similar condition with some constant $\widehat{c}_{*}^{(+)}$for $|t| \leq t_{+}^{0}$ (for instance, we can put $\widehat{c}_{*}^{(+)}=c_{*}^{(+)}\left\|M_{+}\right\|^{-2}$ ). As before, we assume that $A(t)$ satisfies (1.21). Let $\widehat{Z}_{+, Q_{+}}$be the analog of the operator $\widehat{Z}_{Q}$ for the family $\widehat{A}_{+}(t)$ and the operator $Q_{+}$. We assume that the following condition is satisfied.

Condition 1.3. 1) The families $\widehat{A}(t)$ and $\widehat{A}_{+}(t)$ are threshold equivalent. 2) The blocks of the operators $Q$ and $Q_{+}$in $\widehat{\mathfrak{N}}=\widehat{\mathfrak{N}}_{+}$coincide. 3) The operator $\widehat{Z}_{+, Q_{+}}$is equal to zero.

Now we formulate Theorem 6.7 of [BSu5].

Theorem 1.4. Let $\widehat{A}(t), A(t), \widehat{A}_{+}(t)$, and $A_{+}(t)$ be the operator families defined above. Suppose that $A(t)$ and $A_{+}(t)$ satisfy conditions of the form (1.21) and that Condition 1.3 is fulfilled. Let $\widehat{Z}_{Q}$ and $\widehat{N}_{Q}$ be the operators defined above (see (1.35) and (1.37)). Then, for $|t| \leq \min \left\{t^{0}, t_{+}^{0}\right\}$, we have

$$
\left(\widehat{A}(t)+\varepsilon^{2} Q\right)^{-1}=\widehat{R}_{+}(t, \varepsilon)+t \widehat{Z}_{Q} \widehat{R}_{+}(t, \varepsilon)+t \widehat{R}_{+}(t, \varepsilon) \widehat{Z}_{Q}^{*}-t^{3} \widehat{R}_{+}(t, \varepsilon) \widehat{N}_{Q} \widehat{R}_{+}(t, \varepsilon)+\check{\mathcal{J}},
$$

where $\widehat{R}_{+}(t, \varepsilon)=\left(\widehat{A}_{+}(t)+\varepsilon^{2} Q_{+}\right)^{-1}$, and

$$
\begin{aligned}
\|\check{\mathcal{J}}\|_{\mathfrak{H} \rightarrow \mathfrak{H}} \leq \check{C}= & C\left\|Q^{-1}\right\| \\
& +C_{+}\left\|Q_{+}^{-1}\right\|\left(1+2^{-1 / 2}\|Q\|^{1 / 2}\left\|Q^{-1}\right\|^{1 / 2}\left(1+2\left(\widehat{c}_{*}^{(+)}\right)^{-1}\left\|\widehat{X}_{1}\right\|^{2}\right)\right) .
\end{aligned}
$$

Here the constant $C$ is defined by (1.25), and $C_{+}$is the analog of $C$ for the family $A_{+}(t)$.

If $\widehat{Z}_{Q}=0$ (and then also $\widehat{N}_{Q}=0$ ), we obtain the following result (it is implied by [BSu5, (6.22), (6.26)-(6.28)]).

Theorem 1.5. Under the conditions of Theorem 1.4, suppose that $\widehat{Z}_{Q}=0$. Then

$$
\begin{array}{r}
\left\|\left(\widehat{A}(t)+\varepsilon^{2} Q\right)^{-1}-\left(\widehat{A}_{+}(t)+\varepsilon^{2} Q_{+}\right)^{-1}\right\|_{\mathfrak{H} \rightarrow \mathfrak{H}} \leq C\left\|Q^{-1}\right\|+C_{+}\left\|Q_{+}^{-1}\right\|, \\
|t| \leq \min \left\{t^{0}, t_{+}^{0}\right\}, \quad \varepsilon>0 .
\end{array}
$$


Remark 1.6. Let $\widehat{Z}$ be the analog of the operator $Z$ for $\widehat{A}(t)$, i.e., $\widehat{Z}$ is the operator in $\widehat{\mathfrak{H}}$ that takes an element $\widehat{u} \in \widehat{\mathfrak{H}}$ to the solution $\widehat{\psi} \in \widehat{\mathfrak{H}}$ of the problem

$$
\widehat{X}_{0}^{*}\left(\widehat{X}_{0} \widehat{\psi}+\widehat{X}_{1} \widehat{\omega}\right)=0, \quad \widehat{\psi} \perp \widehat{\mathfrak{N}},
$$

where $\widehat{\omega}=\widehat{P} \widehat{u}$. Clearly, the conditions $\widehat{Z}=0$ and $\widehat{Z}_{Q}=0$ are equivalent.

1.9. Representation for the resolvent. A representation for $\left(A(t)+\varepsilon^{2} I\right)^{-1}$ directly follows from Theorem 1.4 and relation (1.48) (see [BSu5, Theorem 6.8]).

Theorem 1.7. Under the conditions of Theorem 1.4, we have

$$
\begin{aligned}
\left(A(t)+\varepsilon^{2} I\right)^{-1}=M^{-1}\left(\widehat{R}_{+}(t, \varepsilon)+\right. & t\left(\widehat{Z}_{Q} \widehat{R}_{+}(t, \varepsilon)+\widehat{R}_{+}(t, \varepsilon) \widehat{Z}_{Q}^{*}\right) \\
& \left.-t^{3} \widehat{R}_{+}(t, \varepsilon) \widehat{N}_{Q} \widehat{R}_{+}(t, \varepsilon)+\check{\mathcal{J}}\right)\left(M^{*}\right)^{-1},
\end{aligned}
$$

where $\check{\mathcal{J}}$ admits estimate (1.49).

The following theorem is a consequence of Theorem 1.5 and identity (1.48).

Theorem 1.8. Under the conditions of Theorem 1.5, we have

$$
\begin{aligned}
& \left\|\left(A(t)+\varepsilon^{2} I\right)^{-1}-M^{-1}\left(\widehat{A}_{+}(t)+\varepsilon^{2} Q_{+}\right)^{-1}\left(M^{*}\right)^{-1}\right\|_{\mathfrak{H} \rightarrow \mathfrak{H}} \\
& \quad \leq\left(C\left\|Q^{-1}\right\|+C_{+}\left\|Q_{+}^{-1}\right\|\right)\|Q\|, \quad|t| \leq \min \left\{t^{0}, t_{+}^{0}\right\}, \quad \varepsilon>0 .
\end{aligned}
$$

In fact, the material of Subsection 1.9 will not be used in the sequel. However, here we have demonstrated a simple trick to be used systematically in $\S 9$ in specific situations.

\section{§2. Periodic Differential operators in $L_{2}\left(\mathbb{R}^{d} ; \mathbb{C}^{n}\right)$. DIRECT INTEGRAL EXPANSION}

Here we describe the class of matrix second-order DOs admitting a factorization of the form $\mathcal{A}=\mathcal{X}^{*} \mathcal{X}$, where $\mathcal{X}$ is a homogeneous first-order DO. This class was distinguished and studied in [BSu1, BSu2].

2.1. Second-order operators admitting factorization. Let $b(\mathbf{D}): L_{2}\left(\mathbb{R}^{d} ; \mathbb{C}^{n}\right) \rightarrow$ $L_{2}\left(\mathbb{R}^{d} ; \mathbb{C}^{m}\right)$ be a homogeneous first-order DO with constant coefficients. We always assume that $m \geq n$. Let $\widetilde{\mathbf{e}}_{1}, \ldots, \widetilde{\mathbf{e}}_{n}$ be the standard (orthonormal) basis in $\mathbb{C}^{n}$. Similarly, $\mathbf{e}_{1}, \ldots, \mathbf{e}_{m}$ is the standard basis in $\mathbb{C}^{m}$, and $\mathbf{e}_{1}^{\prime}, \ldots, \mathbf{e}_{d}^{\prime}$ is the standard basis in $\mathbb{R}^{d}$. Then the symbol $b(\boldsymbol{\xi})$ is associated with the operator $b(\mathbf{D})$. This symbol is an $(m \times n)$-matrixvalued linear homogeneous function of $\xi \in \mathbb{R}^{d}$. Suppose that the rank of the symbol is maximal, i.e.,

$$
\operatorname{rank} b(\xi)=n, \quad 0 \neq \xi \in \mathbb{R}^{d} .
$$

Then

$$
\alpha_{0} \mathbf{1}_{n} \leq b(\boldsymbol{\theta})^{*} b(\boldsymbol{\theta}) \leq \alpha_{1} \mathbf{1}_{n}, \quad|\boldsymbol{\theta}|=1, \quad 0<\alpha_{0} \leq \alpha_{1}<\infty,
$$

with some constants $\alpha_{0}$ and $\alpha_{1}$.

Suppose that an $(n \times n)$-matrix-valued function $f(\mathbf{x})$ and an $(m \times m)$-matrix-valued function $h(\mathbf{x}), \mathbf{x} \in \mathbb{R}^{d}$, are bounded together with their inverses:

$$
f, f^{-1} \in L_{\infty}\left(\mathbb{R}^{d}\right) ; \quad h, h^{-1} \in L_{\infty}\left(\mathbb{R}^{d}\right) .
$$

We consider the DO

$$
\begin{aligned}
\mathcal{X} & :=h b(\mathbf{D}) f: L_{2}\left(\mathbb{R}^{d} ; \mathbb{C}^{n}\right) \rightarrow L_{2}\left(\mathbb{R}^{d} ; \mathbb{C}^{m}\right), \\
\operatorname{Dom} \mathcal{X} & :=\left\{\mathbf{u} \in L_{2}\left(\mathbb{R}^{d} ; \mathbb{C}^{n}\right): f \mathbf{u} \in H^{1}\left(\mathbb{R}^{d} ; \mathbb{C}^{n}\right)\right\}
\end{aligned}
$$


The operator (2.4) is closed on the domain (2.5). The selfadjoint operator

$$
\mathcal{A}:=\mathcal{X}^{*} \mathcal{X}
$$

in $L_{2}\left(\mathbb{R}^{d} ; \mathbb{C}^{n}\right)$ is generated by the closed quadratic form

$$
a[\mathbf{u}, \mathbf{u}]:=\|\mathcal{X} \mathbf{u}\|_{L_{2}\left(\mathbb{R}^{d} ; \mathbb{C}^{m}\right)}^{2}, \quad \mathbf{u} \in \operatorname{Dom} \mathcal{X} .
$$

Formally,

$$
\mathcal{A}=f(\mathbf{x})^{*} b(\mathbf{D})^{*} g(\mathbf{x}) b(\mathbf{D}) f(\mathbf{x}), \quad g(\mathbf{x}):=h(\mathbf{x})^{*} h(\mathbf{x}) .
$$

By using the Fourier transformation and conditions (2.2) and (2.3), it is easy to show that

$$
c_{0} \int_{\mathbb{R}^{d}}|\mathbf{D}(f \mathbf{u})|^{2} d \mathbf{x} \leq a[\mathbf{u}, \mathbf{u}] \leq c_{1} \int_{\mathbb{R}^{d}}|\mathbf{D}(f \mathbf{u})|^{2} d \mathbf{x}, \quad \mathbf{u} \in \operatorname{Dom} \mathcal{X}
$$

where

$$
c_{0}=\alpha_{0}\left\|h^{-1}\right\|_{L_{\infty}}^{-2}, \quad c_{1}=\alpha_{1}\|h\|_{L_{\infty}}^{2} .
$$

2.2. The lattices $\Gamma$ and $\widetilde{\Gamma}$. In what follows, the functions $f$ and $h$ are assumed to be periodic with respect to some lattice $\Gamma \subset \mathbb{R}^{d}$. Let $\mathbf{a}_{1}, \ldots, \mathbf{a}_{d} \in \mathbb{R}^{d}$ be the basis in $\mathbb{R}^{d}$ that generates the lattice $\Gamma$ :

$$
\Gamma=\left\{\mathbf{a} \in \mathbb{R}^{d}: \mathbf{a}=\sum_{j=1}^{d} \nu^{j} \mathbf{a}_{j}, \nu^{j} \in \mathbb{Z}\right\},
$$

and let $\Omega$ be the (elementary) cell of $\Gamma$ :

$$
\Omega:=\left\{\mathbf{x} \in \mathbb{R}^{d}: \mathbf{x}=\sum_{j=1}^{d} \tau^{j} \mathbf{a}_{j}, 0<\tau^{j}<1\right\} .
$$

The basis $\mathbf{b}^{1}, \ldots, \mathbf{b}^{d}$ in $\mathbb{R}^{d}$ dual to $\mathbf{a}_{1}, \ldots, \mathbf{a}_{d}$ is defined by the relations $\left\langle\mathbf{b}^{i}, \mathbf{a}_{j}\right\rangle=2 \pi \delta_{j}^{i}$. This basis gives rise to the lattice $\widetilde{\Gamma}$ dual to $\Gamma$ :

$$
\widetilde{\Gamma}=\left\{\mathbf{b} \in \mathbb{R}^{d}: \mathbf{b}=\sum_{i=1}^{d} \rho_{i} \mathbf{b}^{i}, \rho_{i} \in \mathbb{Z}\right\} .
$$

The cell of the lattice $\widetilde{\Gamma}$ can be defined by analogy with (2.8). However, it is more convenient to consider the Brillouin zone

$$
\widetilde{\Omega}=\left\{\mathbf{k} \in \mathbb{R}^{d}:|\mathbf{k}|<|\mathbf{k}-\mathbf{b}|, 0 \neq \mathbf{b} \in \widetilde{\Gamma}\right\} .
$$

The domain $\widetilde{\Omega}$ is also a fundamental domain of $\widetilde{\Gamma}$. We use the notation $|\Omega|=$ meas $\Omega$, $|\widetilde{\Omega}|=$ meas $\widetilde{\Omega}$. Note that $|\Omega||\widetilde{\Omega}|=(2 \pi)^{d}$.

Let $r_{0}$ be the radius of the ball inscribed in $\operatorname{clos} \widetilde{\Omega}$. We use the notation $\mathcal{B}(r)=$ $\left\{\mathbf{k} \in \mathbb{R}^{d}:|\mathbf{k}| \leq r\right\}, 0<r \leq r_{0}$.

Below, $\widetilde{W}_{p}^{s}(\Omega)$ stands for the subspace of all functions $f$ in $W_{p}^{s}(\Omega)$ such that the $\Gamma$ periodic extension of $f$ to $\mathbb{R}^{d}$ belongs to $W_{p, \text { loc }}^{s}\left(\mathbb{R}^{d}\right)$. For $p=2$, this space is denoted by $\widetilde{H}^{s}(\Omega)$. 
2.3. The Gelfand transformation. Initially, the Gelfand transformation $\mathcal{V}$ is defined on the functions $\mathbf{v}$ belonging to the Schwartz class $\mathcal{S}\left(\mathbb{R}^{d} ; \mathbb{C}^{n}\right)$ by the formula

$$
\widetilde{\mathbf{v}}(\mathbf{k}, \mathbf{x})=(\mathcal{V} \mathbf{v})(\mathbf{k}, \mathbf{x})=|\widetilde{\Omega}|^{-1 / 2} \sum_{\mathbf{a} \in \Gamma} \exp (-i\langle\mathbf{k}, \mathbf{x}+\mathbf{a}\rangle) \mathbf{v}(\mathbf{x}+\mathbf{a}), \quad \mathbf{x} \in \Omega, \mathbf{k} \in \widetilde{\Omega},
$$

and then $\mathcal{V}$ extends by continuity up to a unitary mapping

$$
\mathcal{V}: L_{2}\left(\mathbb{R}^{d} ; \mathbb{C}^{n}\right) \rightarrow \int_{\widetilde{\Omega}} \oplus L_{2}\left(\Omega ; \mathbb{C}^{n}\right) d \mathbf{k}=: \mathcal{K}
$$

The relation $\mathbf{v} \in H^{1}\left(\mathbb{R}^{d} ; \mathbb{C}^{n}\right)$ is equivalent to the condition that $\widetilde{\mathbf{v}}(\mathbf{k}, \cdot) \in \widetilde{H}^{1}\left(\Omega ; \mathbb{C}^{n}\right)$ for almost every $\mathbf{k} \in \widetilde{\Omega}$ and

$$
\int_{\widetilde{\Omega}} \int_{\Omega}\left(|(\mathbf{D}+\mathbf{k}) \widetilde{\mathbf{v}}(\mathbf{k}, \mathbf{x})|^{2}+|\widetilde{\mathbf{v}}(\mathbf{k}, \mathbf{x})|^{2}\right) d \mathbf{x} d \mathbf{k}<\infty .
$$

Under the transformation $\mathcal{V}$, the operator of multiplication by a bounded periodic function in $L_{2}\left(\mathbb{R}^{d} ; \mathbb{C}^{n}\right)$ turns into multiplication by the same function on the fibers of the direct integral $\mathcal{K}$ (see $(2.10)$ ). On these fibers, the operator $b(\mathbf{D})$ applied to $\mathbf{v} \in H^{1}\left(\mathbb{R}^{d} ; \mathbb{C}^{n}\right)$ turns into the operator $b(\mathbf{D}+\mathbf{k})$ applied to $\widetilde{\mathbf{v}}(\mathbf{k}, \cdot) \in \widetilde{H}^{1}\left(\Omega ; \mathbb{C}^{n}\right)$.

2.4. The forms $a(\mathbf{k})$ and the operators $\mathcal{A}(\mathbf{k})$. Putting

$$
\mathfrak{H}=L_{2}\left(\Omega ; \mathbb{C}^{n}\right), \quad \mathfrak{H}_{*}=L_{2}\left(\Omega ; \mathbb{C}^{m}\right),
$$

we consider the closed operator $\mathcal{X}(\mathbf{k}): \mathfrak{H} \rightarrow \mathfrak{H}_{*}, \mathbf{k} \in \mathbb{R}^{d}$, defined on the domain

$$
\mathfrak{d}:=\operatorname{Dom} \mathcal{X}(\mathbf{k})=\left\{\mathbf{u} \in \mathfrak{H}: f \mathbf{u} \in \widetilde{H}^{1}\left(\Omega ; \mathbb{C}^{n}\right)\right\}
$$

by the formula

$$
\mathcal{X}(\mathbf{k})=h b(\mathbf{D}+\mathbf{k}) f .
$$

The selfadjoint operator

$$
\mathcal{A}(\mathbf{k}):=\mathcal{X}(\mathbf{k})^{*} \mathcal{X}(\mathbf{k}): \mathfrak{H} \rightarrow \mathfrak{H}
$$

is generated by the closed quadratic form

$$
a(\mathbf{k})[\mathbf{u}, \mathbf{u}]:=\|\mathcal{X}(\mathbf{k}) \mathbf{u}\|_{\mathfrak{H}_{*}}^{2}, \quad \mathbf{u} \in \mathfrak{d} .
$$

It is easily seen (cf. [BSu2, (2.2.6)]) that

$$
c_{0} \int_{\Omega}|(\mathbf{D}+\mathbf{k}) \mathbf{v}|^{2} d \mathbf{x} \leq a(\mathbf{k})[\mathbf{u}, \mathbf{u}] \leq c_{1} \int_{\Omega}|(\mathbf{D}+\mathbf{k}) \mathbf{v}|^{2} d \mathbf{x}, \quad \mathbf{v}=f \mathbf{u} \in \widetilde{H}^{1}\left(\Omega ; \mathbb{C}^{n}\right),
$$

where $c_{0}$ and $c_{1}$ are defined by (2.7). From (2.11) and the fact that the embedding of $\widetilde{H}^{1}\left(\Omega ; \mathbb{C}^{n}\right)$ in $\mathfrak{H}$ is compact, it follows that the spectrum of $\mathcal{A}(\mathbf{k})$ is discrete. Observe that the resolvent of the operator $\mathcal{A}(\mathbf{k})$ is compact and depends on $\mathbf{k} \in \mathbb{R}^{d}$ continuously (in the operator norm). Let

$$
\mathfrak{N}:=\operatorname{Ker} \mathcal{A}(0)=\operatorname{Ker} \mathcal{X}(0) .
$$

Relations (2.11) with $\mathbf{k}=0$ show that

$$
\mathfrak{N}=\left\{\mathbf{u} \in L_{2}\left(\Omega ; \mathbb{C}^{n}\right): f \mathbf{u}=\mathbf{c} \in \mathbb{C}^{n}\right\}, \quad \operatorname{dim} \mathfrak{N}=n .
$$

Note that $\mathfrak{N}$ is independent of the matrix $g$. 
2.5. The direct integral for the operator $\mathcal{A}$. The operators $\mathcal{A}(\mathbf{k})$ allow us to partially diagonalize the operator $\mathcal{A}$ in the direct integral $\mathcal{K}$ (see (2.10)). Let $\widetilde{\mathbf{u}}=\mathcal{V} \mathbf{u}$, $\mathbf{u} \in \operatorname{Dom} a$. Then

$$
\begin{gathered}
\widetilde{\mathbf{u}}(\mathbf{k}, \cdot) \in \mathfrak{d} \quad \text { for a.e. } \mathbf{k} \in \mathbb{R}^{d}, \\
a[\mathbf{u}, \mathbf{u}]=\int_{\widetilde{\Omega}} a(\mathbf{k})[\widetilde{\mathbf{u}}(\mathbf{k}, \cdot), \widetilde{\mathbf{u}}(\mathbf{k}, \cdot)] d \mathbf{k} .
\end{gathered}
$$

Conversely, if $\widetilde{\mathbf{u}} \in \mathcal{K}$ satisfies (2.14) and the integral in (2.15) is finite, then $\mathbf{u} \in \operatorname{Dom} a$ and (2.15) is valid. The above arguments show that, in the direct integral $\mathcal{K}$, the operator $\mathcal{A}$ turns into multiplication by the operator-valued function $\mathcal{A}(\mathbf{k}), \mathbf{k} \in \widetilde{\Omega}$. All this can be expressed briefly by the formula

$$
\mathcal{V} \mathcal{A} \mathcal{V}^{-1}=\int_{\widetilde{\Omega}} \oplus \mathcal{A}(\mathbf{k}) d \mathbf{k}
$$

2.6. Incorporation of the operators $\mathcal{A}(\mathbf{k})$ into the pattern of $\S 1$. For $\mathbf{k} \in \mathbb{R}^{d}$, we put $\mathbf{k}=t \boldsymbol{\theta}, t=|\mathbf{k}|,|\boldsymbol{\theta}|=1$, and view $t$ as the perturbation parameter. At the same time, all the constructions will depend on the additional parameter $\boldsymbol{\theta}$.

We shall apply the method described in $\S 1$, putting $\mathfrak{H}=L_{2}\left(\Omega ; \mathbb{C}^{n}\right), \mathfrak{H}_{*}=L_{2}\left(\Omega ; \mathbb{C}^{m}\right)$. The role of $X(t)$ is played by the operator

$$
\begin{aligned}
X(t, \boldsymbol{\theta}) & =\mathcal{X}(t \boldsymbol{\theta})=X_{0}+t X_{1}(\boldsymbol{\theta}), \\
X_{0} & =\mathcal{X}(0)=h b(\mathbf{D}) f, \quad \operatorname{Dom} X_{0}=\mathfrak{d} ; \quad X_{1}(\boldsymbol{\theta})=h b(\boldsymbol{\theta}) f .
\end{aligned}
$$

Finally, the role of $A(t)$ is played by the operator $A(t, \boldsymbol{\theta})=\mathcal{A}(t \boldsymbol{\theta})$. In accordance with (2.12) and (2.13), $\mathfrak{N}=\operatorname{Ker} X_{0}=\operatorname{Ker} \mathcal{X}(0), \operatorname{dim} \mathfrak{N}=n$. The condition $m \geq n$ ensures that $n \leq n_{*}$. The following alternative realizes: either $n_{*}=\infty$ (if $m>n$ ), or $n_{*}=n$ (if $m=n$ ); see [BSu2, §2.3]. By variational estimates, in [BSu2, $\$ 2.2,2.3$ ] it was shown that the distance $d^{0}$ from the point $\lambda=0$ to the rest of the spectrum of $\mathcal{A}(0)$ satisfies the following lower estimate:

$$
d^{0} \geq 4 c_{*} r_{0}^{2}, \quad c_{*}=\alpha_{0}\left\|f^{-1}\right\|_{L_{\infty}}^{-2}\left\|h^{-1}\right\|_{L_{\infty}}^{-2} .
$$

In Subsection 1.1, it was required to choose $\delta<d^{0} / 8$. Taking account of (2.17), we fix $\delta$ as follows:

$$
\delta=c_{*} r_{0}^{2} / 4=\left(r_{0} / 2\right)^{2} \alpha_{0}\left\|f^{-1}\right\|_{L_{\infty}}^{-2}\left\|h^{-1}\right\|_{L_{\infty}}^{-2} .
$$

Next, the estimate $\left\|X_{1}(\boldsymbol{\theta})\right\| \leq \alpha_{1}^{1 / 2}\|f\|_{L_{\infty}}\|h\|_{L_{\infty}}$ allows us to choose $t^{0}$ equal not to $\delta^{1 / 2}\left\|X_{1}(\boldsymbol{\theta})\right\|^{-1}$ (see (1.2)) but to a smaller number independent of $\boldsymbol{\theta}$. Namely, we put

$$
\begin{aligned}
t^{0} & =\delta^{1 / 2} \alpha_{1}^{-1 / 2}\|f\|_{L_{\infty}}^{-1}\|h\|_{L_{\infty}}^{-1} \\
& =\left(r_{0} / 2\right) \alpha_{0}^{1 / 2} \alpha_{1}^{-1 / 2}\|f\|_{L_{\infty}}^{-1}\left\|f^{-1}\right\|_{L_{\infty}}^{-1}\|h\|_{L_{\infty}}^{-1}\left\|h^{-1}\right\|_{L_{\infty}}^{-1} .
\end{aligned}
$$

Observe that (2.19) implies the inequality

$$
t^{0} \leq r_{0} / 2
$$

Thus, $\mathcal{B}\left(t^{0}\right) \subset \mathcal{B}\left(r_{0} / 2\right) \subset \widetilde{\Omega}$. In what follows, we always assume that $c_{*}$, $\delta$, and $t^{0}$ are defined by (2.17)-(2.19).

Below we shall use the following estimate, which can easily be proved by variational estimates for the eigenvalues (see [BSu2, (2.2.13)]):

$$
\mathcal{A}(\mathbf{k})=A(t, \boldsymbol{\theta}) \geq c_{*} r^{2} I, \quad \mathbf{k} \in \operatorname{clos} \widetilde{\Omega} \backslash \mathcal{B}(r), \quad 0<r \leq r_{0} .
$$


2.7. Nondegeneracy of the germ of the family $A(t, \boldsymbol{\theta})$. The analytic (in $t$ ) branches of the eigenvalues $\lambda_{l}(t, \theta)$ (see (1.7) and (1.8)) and the branches of the eigenvectors $\varphi_{l}(t, \theta), l=1, \ldots, n,|t| \leq t^{0}$, depend on $\boldsymbol{\theta}$. In BSu2, $\left.\S \S 2.2,2.3\right]$, by variational estimates for the eigenvalues, it was shown that

$$
\lambda_{l}(t, \boldsymbol{\theta}) \geq c_{*} t^{2}, \quad l=1, \ldots, n, \quad t \in\left[0, t^{0}\right] .
$$

It is essential that $c_{*}$ and $t^{0}$ in (2.21) do not depend on $\theta$. Inequality (2.21) implies that the germ $S(\boldsymbol{\theta})$ of the family $A(t, \boldsymbol{\theta})$ is nondegenerate uniformly in $\boldsymbol{\theta}$.

\section{§3. The EFfective Matrix AND the EFFeCtive OPERAtor}

3.1. The case where $f=\mathbf{1}_{n}$. In [BSu2, $\left.\S 3.1\right]$, the spectral germ $S(\boldsymbol{\theta})$ of the operator family $A(t, \boldsymbol{\theta})$ was constructed. It is convenient to start with the case where $f=\mathbf{1}_{n}$. In this case, we agree to mark all the corresponding objects by the upper symbol "^". Next, the dependence of the objects on the coefficients will be indicated explicitly in the notation. For instance, for the operator $\widehat{\mathcal{A}}=\widehat{\mathcal{A}}(g)=b(\mathbf{D})^{*} g(\mathbf{x}) b(\mathbf{D})$ the family $\widehat{\mathcal{A}}(\mathbf{k})=\widehat{\mathcal{A}}(\mathbf{k} ; g)$ is denoted by $\widehat{A}(t, \boldsymbol{\theta})=\widehat{A}(t, \boldsymbol{\theta} ; g)$. If $f=\mathbf{1}_{n}$, the kernel (2.13) takes the form

$$
\widehat{\mathfrak{N}}=\left\{\mathbf{u} \in \mathfrak{H}: \mathbf{u}=\mathbf{c} \in \mathbb{C}^{n}\right\} .
$$

Let $\widehat{P}$ be the orthogonal projection of $\mathfrak{H}$ onto the subspace (3.1). Then

$$
\widehat{P} \mathbf{u}=|\Omega|^{-1} \int_{\Omega} \mathbf{u}(\mathbf{x}) d \mathbf{x}, \quad \mathbf{u} \in \mathfrak{H} .
$$

In other words, $\widehat{P}$ is the operator of averaging over the cell.

By [BSu2, §3.1], the spectral germ $\widehat{S}(\boldsymbol{\theta})$ of the family $\widehat{A}(t, \boldsymbol{\theta} ; g)$ acts in $\widehat{\mathfrak{N}}$ and is represented as

$$
\widehat{S}(\boldsymbol{\theta})=b(\boldsymbol{\theta})^{*} g^{0} b(\boldsymbol{\theta}), \quad \boldsymbol{\theta} \in \mathbb{S}^{d-1} .
$$

Here $g^{0}$ is the so-called effective matrix.

To describe the constant positive $(m \times m)$-matrix $g^{0}$, we introduce the operator $\Lambda$ : $\mathbb{C}^{m} \rightarrow \mathfrak{H}$ that takes a vector $\mathbf{C} \in \mathbb{C}^{m}$ to the (weak) periodic solution $\mathbf{v}_{\mathbf{C}} \in \widetilde{H}^{1}\left(\Omega ; \mathbb{C}^{n}\right)$ of the equation

$$
b(\mathbf{D})^{*} g(\mathbf{x})\left(b(\mathbf{D}) \mathbf{v}_{\mathbf{C}}(\mathbf{x})+\mathbf{C}\right)=0, \quad \int_{\Omega} \mathbf{v}_{\mathbf{C}} d \mathbf{x}=0 .
$$

Recall that, in Subsection 2.1, we fixed the bases $\mathbf{e}_{1}, \ldots, \mathbf{e}_{m}$ in $\mathbb{C}^{m}$ and $\widetilde{\mathbf{e}}_{1}, \ldots, \widetilde{\mathbf{e}}_{n}$ in $\mathbb{C}^{n}$. The solution $\mathbf{v}_{\mathbf{e}_{j}}$ of problem (3.4) with $\mathbf{C}=\mathbf{e}_{j}$ is denoted by $\mathbf{v}_{j}, j=1, \ldots, m$. In the basis $\widetilde{\mathbf{e}}_{1}, \ldots, \widetilde{\mathbf{e}}_{n}$, the vector-valued functions $\mathbf{v}_{j}(\mathbf{x})$ are represented as columns of length $n$. Let $\Lambda(\mathbf{x})$ be the periodic $(n \times m)$-matrix-valued function with the columns $\mathbf{v}_{1}(\mathbf{x}), \ldots, \mathbf{v}_{m}(\mathbf{x})$. Then the operator $\Lambda$ acts as multiplication by the matrix $\Lambda(\mathbf{x})$. Note that the mean value of $\Lambda(\mathbf{x})$ is equal to zero. We introduce the periodic $(m \times m)$-matrixvalued functions $b(\mathbf{D}) \Lambda(\mathbf{x})$ and

$$
\widetilde{g}(\mathbf{x})=g(\mathbf{x})\left(b(\mathbf{D}) \Lambda(\mathbf{x})+\mathbf{1}_{m}\right) .
$$

The effective matrix $g^{0}$ is given by the relation

$$
g^{0}=|\Omega|^{-1} \int_{\Omega} \widetilde{g}(\mathbf{x}) d \mathbf{x} .
$$

Remark 3.1. If the matrices $b(\boldsymbol{\theta})$ and $g(\mathbf{x})$ have real entries, then the solutions $\mathbf{v}_{j}(\mathbf{x})$ and the matrix $\Lambda(\mathbf{x})$ are purely imaginary. Hence, the matrices $\widetilde{g}(\mathbf{x})$ and $g^{0}$ have real entries. 
We put

$$
\widehat{\mathcal{A}}^{0}=\widehat{\mathcal{A}}\left(g^{0}\right)=b(\mathbf{D})^{*} g^{0} b(\mathbf{D}) .
$$

The germ of the corresponding family $\widehat{A}\left(t, \boldsymbol{\theta} ; g^{0}\right)$ coincides with (3.3). Thus, the families $\widehat{A}(t, \boldsymbol{\theta} ; g)$ and $\widehat{A}\left(t, \boldsymbol{\theta} ; g^{0}\right)$ are threshold equivalent for all $\boldsymbol{\theta} \in \mathbb{S}^{d-1}$. The operator $\widehat{\mathcal{A}}^{0}=$ $\widehat{\mathcal{A}}\left(g^{0}\right)$ is called the effective operator for $\widehat{\mathcal{A}}(g)$.

3.2. Properties of the effective matrix. The following properties of the effective matrix were proved in BSu2, Theorem 3.1.5].

Proposition 3.2. The effective matrix satisfies the estimates

$$
\underline{g} \leq g^{0} \leq \bar{g},
$$

where

$$
\bar{g}:=|\Omega|^{-1} \int_{\Omega} g(\mathbf{x}) d \mathbf{x}, \quad \underline{g}^{-1}:=|\Omega|^{-1} \int_{\Omega} g(\mathbf{x})^{-1} d \mathbf{x} .
$$

If $m=n$, then the effective matrix $g^{0}$ coincides with $\underline{g}$ :

$$
g^{0}=\underline{g}, \quad m=n .
$$

For specific DOs, estimates (3.7) are known in homogenization theory as the VoigtReuss bracketing.

We distinguish the cases where one of the inequalities in (3.7) becomes equality. The following statements were obtained in [BSu2, Propositions 3.1.6 and 3.1.7].

Proposition 3.3. The identity $g^{0}=\bar{g}$ is equivalent to the relations

$$
b(\mathbf{D})^{*} \mathbf{g}_{k}(\mathbf{x})=0, \quad k=1, \ldots, m,
$$

where the $\mathbf{g}_{k}(\mathbf{x}), k=1, \ldots, m$, are the columns of the matrix $g(\mathbf{x})$.

Proposition 3.4. The identity $g^{0}=\underline{g}$ is equivalent to the representations

$$
\mathbf{l}_{k}(\mathbf{x})=\mathbf{l}_{k}^{0}+b(\mathbf{D}) \mathbf{w}_{k}, \quad \mathbf{l}_{k}^{0} \in \mathbb{C}^{m}, \quad \mathbf{w}_{k} \in \widetilde{H}^{1}\left(\Omega ; \mathbb{C}^{n}\right), \quad k=1, \ldots, m,
$$

where the $\mathbf{l}_{k}(\mathbf{x}), k=1, \ldots, m$, are the columns of the matrix $g(\mathbf{x})^{-1}$.

Remark 3.5. From (3.10) it follows automatically that $\mathbf{l}_{k}^{0}=\underline{g}^{-1} \mathbf{e}_{k}$ and $g(\mathbf{x})\left(b(\mathbf{D}) \mathbf{w}_{k}+\mathbf{l}_{k}^{0}\right)$ $=\mathbf{e}_{k}, k=1, \ldots, m$. Therefore, up to a constant summand, $\mathbf{w}_{k}$ coincides with the solution $\mathbf{v}_{\mathbf{l}_{k}^{0}}$ of equation (3.4) with $\mathbf{C}=\mathbf{l}_{k}^{0}$. Consequently, the vectors $g(\mathbf{x})\left(b(\mathbf{D}) \mathbf{v}_{\mathbf{C}}+\mathbf{C}\right)$ are constant for all $\mathbf{C} \in \mathbb{C}^{m}$. Then the matrix $\widetilde{g}(\mathbf{x})$ (see (3.5)) is independent of $\mathbf{x}$, i.e., $\widetilde{g}(\mathbf{x})=g^{0}=\underline{g}$.

3.3. The analytic (in $t$ ) branches of the eigenvalues $\widehat{\lambda}_{l}(t, \theta)$ and the branches of the eigenvectors $\widehat{\varphi}_{l}(t, \boldsymbol{\theta})$ of $\widehat{A}(t, \boldsymbol{\theta})$ depend on $\boldsymbol{\theta}$; we indicate this dependence in the notation. The same concerns the coefficients $\widehat{\gamma}_{l}(\boldsymbol{\theta}), \widehat{\mu}_{l}(\boldsymbol{\theta})$, and $\widehat{\omega}_{l}(\boldsymbol{\theta})$ in expansions of the form (1.7), (1.8). By (1.10) and (3.3), the numbers $\widehat{\gamma}_{l}(\boldsymbol{\theta})$ and the vectors $\widehat{\omega}_{l}(\boldsymbol{\theta}) \in \widehat{\mathfrak{N}}$ are, respectively, eigenvalues and eigenvectors of the matrix $b(\boldsymbol{\theta})^{*} g^{0} b(\boldsymbol{\theta})$ :

$$
b(\boldsymbol{\theta})^{*} g^{0} b(\boldsymbol{\theta}) \widehat{\omega}_{l}(\boldsymbol{\theta})=\widehat{\gamma}_{l}(\boldsymbol{\theta}) \widehat{\omega}_{l}(\boldsymbol{\theta}), \quad l=1, \ldots, n .
$$


3.4. The case where $f \neq \mathbf{1}_{n}$. Now, we consider the general operator $\mathcal{A}=\mathcal{A}(g, f)$ of the form (2.6) and the corresponding operator family $A(t, \boldsymbol{\theta})$. Then $\mathcal{A}(g, f)=f^{*} \widehat{\mathcal{A}}(g) f$.

We use the method of Subsection 1.6. Now $\widehat{\mathfrak{H}}=\mathfrak{H}=L_{2}\left(\Omega ; \mathbb{C}^{n}\right)$, the role of the family $\widehat{A}(t)$ is played by $\widehat{A}(t, \boldsymbol{\theta} ; g)$, and the role of $A(t)$ is played by $A(t, \boldsymbol{\theta} ; g, f)=f^{*} \widehat{A}(t, \boldsymbol{\theta} ; g) f$. The isomorphism $M$ is multiplication by the matrix-valued function $f$. The kernel $\mathfrak{N}=$ Ker $X_{0}$ is defined by formula (2.13); it does not depend on $b$ and $g$. The kernel $\widehat{\mathfrak{N}}$ is defined by (3.1). Next, the operator (1.30) is realized as multiplication by the matrixvalued function $Q(\mathbf{x})=\left(f(\mathbf{x}) f(\mathbf{x})^{*}\right)^{-1}$. The block $Q_{\widehat{\mathfrak{N}}}$ is multiplication by the constant matrix $\bar{Q}=\left(\underline{f f^{*}}\right)^{-1}$. By (1.32), the germ $S(\boldsymbol{\theta})$ of the family $A(t, \boldsymbol{\theta})$ and the germ $\widehat{S}(\boldsymbol{\theta})$ of the family $\widehat{A}(t, \boldsymbol{\theta})$ satisfy the relation $S(\boldsymbol{\theta})=\left.f^{-1}(\bar{Q})^{-1} \widehat{S}(\boldsymbol{\theta}) f\right|_{\mathfrak{N}}$.

With the operator $\mathcal{A}(g, f)=f^{*} \widehat{\mathcal{A}}(g) f$, we associate the operator $\mathcal{A}\left(g^{0}, f\right)=f^{*} \widehat{\mathcal{A}}\left(g^{0}\right) f$ $=f^{*} b(\mathbf{D})^{*} g^{0} b(\mathbf{D}) f$ acting in $L_{2}\left(\mathbb{R}^{d} ; \mathbb{C}^{n}\right)$. The corresponding families $A(t, \boldsymbol{\theta} ; g, f)$ and $A\left(t, \boldsymbol{\theta} ; g^{0}, f\right)$ are threshold equivalent for all $\boldsymbol{\theta} \in \mathbb{S}^{d-1}$. However, it is impossible to find an operator with constant coefficients such that the corresponding family is threshold equivalent to the family $A(t, \boldsymbol{\theta} ; g, f)$.

The analytic (in $t$ ) branches of the eigenvalues $\lambda_{l}(t, \theta)$ and the branches of the eigenvectors $\varphi_{l}(t, \boldsymbol{\theta})$ of $A(t, \boldsymbol{\theta})$ admit expansions of the form (1.7) and (1.8) with the coefficients $\gamma_{l}(\boldsymbol{\theta}), \mu_{l}(\boldsymbol{\theta})$, and $\omega_{l}(\boldsymbol{\theta})$. By (1.33) and (3.3), the numbers $\gamma_{l}(\boldsymbol{\theta})$ and the vectors $\widehat{\phi}_{l}(\boldsymbol{\theta})=f \omega_{l}(\boldsymbol{\theta}) \in \widehat{\mathfrak{N}}$ are eigenvalues and eigenvectors of the "generalized" spectral problem for the matrix $b(\boldsymbol{\theta})^{*} g^{0} b(\boldsymbol{\theta})$ :

$$
b(\boldsymbol{\theta})^{*} g^{0} b(\boldsymbol{\theta}) \widehat{\phi}_{l}(\boldsymbol{\theta})=\gamma_{l}(\boldsymbol{\theta}) \bar{Q} \widehat{\phi}_{l}(\boldsymbol{\theta}), \quad l=1, \ldots, n .
$$

\section{Chapter 2. Threshold approximations For Resolvents OF DIFFERENTIAL OPERATORS}

Here we apply the abstract method to the periodic differential operators $\widehat{\mathcal{A}}(g)=$ $b(\mathbf{D})^{*} g b(\mathbf{D})$. We consider the resolvent $\left(\widehat{\mathcal{A}}(g)+\varepsilon^{2} I\right)^{-1}$, as well as the generalized resolvent (Q-resolvent) $\left(\widehat{\mathcal{A}}(g)+\varepsilon^{2} Q\right)^{-1}$. We realize all the objects of the general pattern and obtain the main statements about approximation of the resolvents for small $\varepsilon>0$. Here, the corrector is taken into account, which is our main goal in the entire work. First of all, in $\S \S 4$ and 5 we consider the resolvent and the $Q$-resolvent of the operators $\widehat{\mathcal{A}}(\mathbf{k} ; g)$ acting on the fibers of the direct integral $\mathcal{K}$, i.e., in $L_{2}\left(\Omega ; \mathbb{C}^{n}\right)$. Next, in $\S \S 6$ and 7 , the results obtained are carried over to the resolvents of the operators acting in $L_{2}\left(\mathbb{R}^{d} ; \mathbb{C}^{n}\right)$. The "usual" resolvents are discussed in $\S \S 4$ and 6 , and the $Q$-resolvents are discussed in $\S \S 5$ and 7 . The results of the present chapter are fundamental for the final homogenization results (see Chapter 3).

\section{§4. Approximation of the Resolvent $\left(\widehat{\mathcal{A}}(\mathbf{k})+\varepsilon^{2} I\right)^{-1}$}

4.1. The operators $\widehat{Z}(\mathbf{t})$ and $\widehat{R}(\mathbf{t})$. In accordance with Subsection 1.2, we introduce the operator $\widehat{Z}(\boldsymbol{\theta})$ that plays the role of $Z$ for the family $\widehat{A}(t, \boldsymbol{\theta})$. Let $\mathbf{u} \in \mathfrak{H}$, let $\widehat{P} \mathbf{u}=\mathbf{c}$, and let $\mathbf{w} \in \widetilde{H}^{1}\left(\Omega ; \mathbb{C}^{n}\right)$ be the (weak) solution of the equation

$$
b(\mathbf{D})^{*} g(\mathbf{x})(b(\mathbf{D}) \mathbf{w}+b(\boldsymbol{\theta}) \mathbf{c})=0, \quad \int_{\Omega} \mathbf{w}(\mathbf{x}) d \mathbf{x}=0 .
$$

Here $\widehat{P}$ is the operator of averaging over $\Omega$ introduced in (3.2). Hence, by (1.3) and (1.4), $\mathbf{w}=\widehat{Z}(\boldsymbol{\theta}) \mathbf{u}$. Clearly, the operator $\widehat{Z}(\boldsymbol{\theta})$ and the operator $\Lambda$ introduced in Subsection 3.1 satisfy $\widehat{Z}(\boldsymbol{\theta})=\Lambda b(\boldsymbol{\theta}) \widehat{P}$. Since $\Lambda$ acts as multiplication by the periodic matrix-valued 
function $\Lambda(\mathbf{x})$, we have

$$
\widehat{Z}(\boldsymbol{\theta})=\Lambda(\mathbf{x}) b(\boldsymbol{\theta}) \widehat{P} .
$$

Remark 4.1. In the case where $g^{0}=\bar{g}$, relations (3.9) are satisfied. Then $\mathbf{v}_{j}(\mathbf{x})=0$, whence $\Lambda(\mathbf{x})=0$ and $\widehat{Z}(\boldsymbol{\theta})=0$. In particular, this is true if $g(\mathbf{x})$ is a constant matrix, because $g(\mathbf{x})=g^{0}=\bar{g}$ in this case.

We put $\widehat{Z}(\mathbf{k})=t \widehat{Z}(\boldsymbol{\theta})$. Then

$$
\widehat{Z}(\mathbf{k})=\Lambda(\mathbf{x}) b(\mathbf{k}) \widehat{P}, \quad \mathbf{k} \in \mathbb{R}^{d} .
$$

As in Subsection 1.2, the operator $\widehat{R}(\boldsymbol{\theta})$ that plays the role of $R$ for the family $\widehat{A}(t, \boldsymbol{\theta})$ is given by

$$
\widehat{R}(\boldsymbol{\theta}) \mathbf{c}=h(\mathbf{x})(b(\mathbf{D}) \mathbf{w}+b(\boldsymbol{\theta}) \mathbf{c}), \quad \mathbf{c} \in \widehat{\mathfrak{N}},
$$

where $\mathbf{w}$ is the solution of problem (4.1). In the matrix form, this is equivalent to

$$
\widehat{R}(\boldsymbol{\theta})=h(\mathbf{x})\left(b(\mathbf{D}) \Lambda(\mathbf{x})+\mathbf{1}_{m}\right) b(\boldsymbol{\theta}) .
$$

4.2. The operator $\widehat{N}(\boldsymbol{\theta})$. Now we construct the operator $\widehat{N}(\boldsymbol{\theta})$ that plays the role of $N$ for the family $\widehat{A}(t, \theta)$. By (1.13),

$$
\widehat{N}(\boldsymbol{\theta})=\widehat{Z}(\boldsymbol{\theta})^{*} \widehat{X}_{1}(\boldsymbol{\theta})^{*} \widehat{R}(\boldsymbol{\theta}) \widehat{P}+(\widehat{R}(\boldsymbol{\theta}) \widehat{P})^{*} \widehat{X}_{1}(\boldsymbol{\theta}) \widehat{Z}(\boldsymbol{\theta}) .
$$

Formulas (4.4) and (3.5) show that

$$
\widehat{X}_{1}(\boldsymbol{\theta})^{*} \widehat{R}(\boldsymbol{\theta}) \widehat{P}=b(\boldsymbol{\theta})^{*} h(\mathbf{x})^{*} h(\mathbf{x})\left(b(\mathbf{D}) \Lambda(\mathbf{x})+\mathbf{1}_{m}\right) b(\boldsymbol{\theta}) \widehat{P}=b(\boldsymbol{\theta})^{*} \widetilde{g}(\mathbf{x}) b(\boldsymbol{\theta}) \widehat{P} .
$$

Then, by (4.2) and (4.5),

$$
\widehat{N}(\boldsymbol{\theta})=\widehat{P} b(\boldsymbol{\theta})^{*}\left(\Lambda(\mathbf{x})^{*} b(\boldsymbol{\theta})^{*} \widetilde{g}(\mathbf{x})+\widetilde{g}(\mathbf{x})^{*} b(\boldsymbol{\theta}) \Lambda(\mathbf{x})\right) b(\boldsymbol{\theta}) \widehat{P} .
$$

We introduce the notation

$$
L(\boldsymbol{\theta}):=|\Omega|^{-1} \int_{\Omega}\left(\Lambda(\mathbf{x})^{*} b(\boldsymbol{\theta})^{*} \widetilde{g}(\mathbf{x})+\widetilde{g}(\mathbf{x})^{*} b(\boldsymbol{\theta}) \Lambda(\mathbf{x})\right) d \mathbf{x} .
$$

Observe that $t L(\boldsymbol{\theta})=L(\mathbf{k})$ is a Hermitian $(m \times m)$-matrix-valued function first-order homogeneous in $\mathbf{k} \in \mathbb{R}^{d}$. Thus, $L(\mathbf{k})$ is the symbol of the selfadjoint homogeneous firstorder $D O L(\mathbf{D})$ with constant coefficients.

By (4.7) and (4.8), we have

$$
\widehat{N}(\boldsymbol{\theta})=b(\boldsymbol{\theta})^{*} L(\boldsymbol{\theta}) b(\boldsymbol{\theta}) \widehat{P} .
$$

We put $t^{3} \widehat{N}(\boldsymbol{\theta})=: \widehat{N}(\mathbf{k})$. Then

$$
\widehat{N}(\mathbf{k})=b(\mathbf{k})^{*} L(\mathbf{k}) b(\mathbf{k}) \widehat{P}, \quad \mathbf{k} \in \mathbb{R}^{d} .
$$

The Hermitian matrix-valued function $b(\mathbf{k})^{*} L(\mathbf{k}) b(\mathbf{k})$ is homogeneous in $\mathbf{k}$ of order three. So, this function is the symbol of the homogeneous selfadjoint third-order differential operator $b(\mathbf{D})^{*} L(\mathbf{D}) b(\mathbf{D})$. 
4.3. If $b(\boldsymbol{\theta})$ and $g(\mathbf{x})$ are matrices with real entries, then (see Remark 3.1) the matrix $\widetilde{g}(\mathbf{x})$ has real entries, and $\Lambda(\mathbf{x})$ has purely imaginary entries. Hence, $L(\boldsymbol{\theta})$ and $b(\boldsymbol{\theta})^{*} L(\boldsymbol{\theta}) b(\boldsymbol{\theta})$ are Hermitian matrices with purely imaginary entries. Then, for any real vector $\mathbf{q} \in \widehat{\mathfrak{N}}$ we have $(\widehat{N}(\boldsymbol{\theta}) \mathbf{q}, \mathbf{q})_{\mathfrak{H}}=0$. If the analytic branches of the eigenvalues $\widehat{\lambda}_{l}(t, \boldsymbol{\theta})$ and the branches of the eigenvectors $\widehat{\varphi}_{l}(t, \boldsymbol{\theta})$ of $\widehat{A}(t, \boldsymbol{\theta})$ (see Subsection 3.3) can be chosen so that the vectors $\widehat{\omega}_{1}(\boldsymbol{\theta}), \ldots, \widehat{\omega}_{n}(\boldsymbol{\theta})$ (forming an orthonormal basis in $\widehat{\mathfrak{N}}$ ) are real, ${ }^{1}$ then, by (1.20),

$$
\widehat{\mu}_{l}(\boldsymbol{\theta})=\left(\widehat{N}(\boldsymbol{\theta}) \widehat{\omega}_{l}(\boldsymbol{\theta}), \widehat{\omega}_{l}(\boldsymbol{\theta})\right)_{\mathfrak{H}}=0, \quad l=1, \ldots, n,
$$

whence

$$
\widehat{\lambda}_{l}(t, \boldsymbol{\theta})=\widehat{\gamma}_{l}(\boldsymbol{\theta}) t^{2}+O\left(t^{4}\right), \quad l=1, \ldots, n .
$$

We arrive at the following statement.

Proposition 4.2. Suppose that the matrices $b(\boldsymbol{\theta})$ and $g(\mathbf{x})$ have real entries and that, in the expansions (1.8) for the analytic branches of the eigenvectors of $\widehat{A}(t, \boldsymbol{\theta})$, the elements $\widehat{\omega}_{l}(\boldsymbol{\theta}), l=1, \ldots, n$, can be chosen to be real. Then in $(1.7)$ we have $\widehat{\mu}_{l}(\boldsymbol{\theta})=0, l=$ $1, \ldots, n$, and relations (4.12) are fulfilled, whence (see (1.16)),

$$
\widehat{N}_{0}(\boldsymbol{\theta})=0 .
$$

Corollary 4.3. Suppose that the matrices $b(\boldsymbol{\theta})$ and $g(\mathbf{x})$ have real entries. If the spectrum of the germ $\widehat{S}(\boldsymbol{\theta})=b(\boldsymbol{\theta})^{*} g^{0} b(\boldsymbol{\theta})$ is simple, then relations (4.12) are fulfilled.

Proof. In the case under consideration, the germ $\widehat{S}(\boldsymbol{\theta})$ is given by the symmetric matrix $b(\boldsymbol{\theta})^{*} g^{0} b(\boldsymbol{\theta})$ with simple spectrum. The eigenvectors $\widehat{\omega}_{1}(\boldsymbol{\theta}), \ldots, \widehat{\omega}_{n}(\boldsymbol{\theta})$ of this matrix are defined uniquely up to phase factors, and in the expansions (1.8) they can be chosen to be real.

Thus, if the matrices $b(\boldsymbol{\theta})$ and $g(\mathbf{x})$ have real entries, then relations (4.12) (and also (4.13)) are valid at least "in general position", i.e., when the multiple eigenvalue splits completely already at the first step of the perturbation theory.

Corollary 4.4. For the operator $\mathbf{D}^{*} g(\mathbf{x}) \mathbf{D}$, where the matrix $g(\mathbf{x})$ has real entries, we have $\widehat{N}(\boldsymbol{\theta})=0$.

Indeed, in this case $n=1$ and, by (4.11), the only matrix element of the operator $\widehat{N}(\boldsymbol{\theta})$ is equal to zero.

Now we distinguish other cases where $\widehat{N}(\boldsymbol{\theta})=0$.

Proposition 4.5. 1) Under conditions (3.9) (i.e., in the case where $g^{0}=\bar{g}$ ), we have $\widehat{Z}(\boldsymbol{\theta})=0$, and then also $\widehat{N}(\boldsymbol{\theta})=0$. 2) Under conditions (3.10) (i.e., in the case where $\left.g^{0}=\underline{g}\right)$, we have $L(\boldsymbol{\theta})=0$, and then also $\widehat{N}(\boldsymbol{\theta})=0$. In particular, this is true if $m=n$.

Proof. Statement 1) follows from Remark 4.1. We prove statement 2). Under conditions (3.10), we have $\widetilde{g}(\mathbf{x})=g^{0}=\underline{g}$ (see Remark 3.5). Hence, since $\int_{\Omega} \Lambda(\mathbf{x}) d \mathbf{x}=0$, (4.8) implies that $L(\boldsymbol{\theta})=0$. Therefore, $\widehat{N}(\boldsymbol{\theta})=0$ by $(4.9)$.

There are examples (see Subsections 10.4, 13.2, and 14.6 below) showing that, in general, the operator $\widehat{N}(\boldsymbol{\theta})$ is not equal to zero for the scalar elliptic operator $\mathbf{D}^{*} g(\mathbf{x}) \mathbf{D}$, where $g(\mathbf{x})$ is a Hermitian matrix with complex entries, as well as for matrix operators of the form $\widehat{\mathcal{A}}(g)=b(\mathbf{D})^{*} g(\mathbf{x}) b(\mathbf{D})$, even if the matrices $b(\boldsymbol{\theta})$ and $g(\mathbf{x})$ have real entries. Here, in the first case the operator $\widehat{N}_{0}(\boldsymbol{\theta})$ is nonzero, and in the second case $\widehat{N}_{*}(\boldsymbol{\theta})$ is nonzero.

\footnotetext{
${ }^{1}$ It remains unclear for the authors whether such a choice is always possible.
} 
4.4. Approximation of the resolvent $\left(\widehat{\mathcal{A}}(\mathbf{k})+\varepsilon^{2} I\right)^{-1}$ for $|\mathbf{k}| \leq \widehat{t}^{0}$. Let $g^{0}$ be the effective matrix, and let $\widehat{\mathcal{A}}^{0}=\widehat{\mathcal{A}}\left(g^{0}\right)=b(\mathbf{D})^{*} g^{0} b(\mathbf{D})$ be the effective operator for $\widehat{\mathcal{A}}(g)$. Then the families $\widehat{A}(t, \boldsymbol{\theta})=\widehat{A}(t, \boldsymbol{\theta} ; g)$ and $\widehat{A}^{0}(t, \boldsymbol{\theta})=\widehat{A}\left(t, \boldsymbol{\theta} ; g^{0}\right)$ are threshold equivalent. Our goal is to apply Theorem 1.1 to the threshold equivalent families $\widehat{A}(t, \boldsymbol{\theta})$ and $\widehat{A}^{0}(t, \boldsymbol{\theta})$. For $\widehat{A}^{0}(t, \boldsymbol{\theta})$ the corresponding operator $\widehat{Z}^{0}(\boldsymbol{\theta})$ is equal to zero (see Remark 4.1).

We trace the constants in the estimates. By $(2.2)$, the operator $\widehat{X}_{1}(\boldsymbol{\theta})=h(\mathbf{x}) b(\boldsymbol{\theta})$ admits the estimate

$$
\left\|\widehat{X}_{1}(\boldsymbol{\theta})\right\| \leq \alpha_{1}^{1 / 2}\|h\|_{L_{\infty}}=\alpha_{1}^{1 / 2}\|g\|_{L_{\infty}}^{1 / 2} .
$$

For the operator $\widehat{A}(t, \boldsymbol{\theta})$, all constants will be marked by the upper symbol "^". In accordance with (2.17)-(2.19) with $f=\mathbf{1}_{n}$, we have

$$
\begin{aligned}
\widehat{c}_{*} & =\alpha_{0}\left\|g^{-1}\right\|_{L_{\infty}}^{-1}, \\
\widehat{\delta} & =\left(r_{0} / 2\right)^{2} \alpha_{0}\left\|g^{-1}\right\|_{L_{\infty}}^{-1}, \\
\widehat{t}^{0} & =\widehat{\delta}^{1 / 2} \alpha_{1}^{-1 / 2}\|g\|_{L_{\infty}}^{-1 / 2} .
\end{aligned}
$$

The inequality $\widehat{t}^{0} \leq r_{0} / 2$ also remains true. Estimates (3.7) for the effective matrix imply that

$$
\left\|g^{0}\right\|_{L_{\infty}} \leq\|g\|_{L_{\infty}}, \quad\left\|\left(g^{0}\right)^{-1}\right\|_{L_{\infty}} \leq\left\|g^{-1}\right\|_{L_{\infty}} .
$$

Combining this with (4.15)-(4.17), we see that if $g$ is replaced by $g^{0}$, the constants $\widehat{c}_{*}, \widehat{\delta}$ and $\widehat{t}^{0}$ can only become larger.

Next, for the operator $\widehat{A}(t, \boldsymbol{\theta})$ the role of the constant (1.25) is played by

$$
\widehat{C}(\boldsymbol{\theta})=\widehat{\delta}^{-1}\left(\beta_{1} \widehat{c}_{*}^{-1}\left\|\widehat{X}_{1}(\boldsymbol{\theta})\right\|^{2}+\beta_{2} \widehat{c}_{*}^{-2}\left\|\widehat{X}_{1}(\boldsymbol{\theta})\right\|^{4}+\beta_{3} \widehat{c}_{*}^{-3}\left\|\widehat{X}_{1}(\boldsymbol{\theta})\right\|^{6}+3^{-1}\right) .
$$

Now it is convenient to use the constant

$$
\widehat{C}=\widehat{\delta}^{-1}\left(\beta_{1} \widehat{c}_{*}^{-1} \alpha_{1}\|g\|_{L_{\infty}}+\beta_{2} \widehat{c}_{*}^{-2} \alpha_{1}^{2}\|g\|_{L_{\infty}}^{2}+\beta_{3} \widehat{c}_{*}^{-3} \alpha_{1}^{3}\|g\|_{L_{\infty}}^{3}+3^{-1}\right)
$$

instead of (4.19). The constant (4.20) fits for all $\boldsymbol{\theta}$. We have used estimate (4.14). Similarly, we may roughen the constant $\widehat{C}^{0}(\boldsymbol{\theta})$, which plays the role of the constant (1.26) for the operator $\widehat{A}^{0}(t, \theta)$. Herewith, the new value of this constant does not exceed $\widehat{C}$. As a result, the constant $\widehat{C}_{*}(\boldsymbol{\theta})$ that realizes $C_{*}$ in $(1.24)$ can be replaced by the constant

$$
\widehat{C}_{*}=\widehat{C}\left(2+2^{-1 / 2}+2^{1 / 2} \widehat{c}_{*}^{-1} \alpha_{1}\|g\|_{L_{\infty}}\right) .
$$

Applying Theorem 1.1, we arrive at the following result.

Theorem 4.6. Let $\widehat{\mathcal{A}}(\mathbf{k})=\widehat{\mathcal{A}}(\mathbf{k} ; g)$ and $\widehat{\mathcal{A}}^{0}(\mathbf{k})=\widehat{\mathcal{A}}\left(\mathbf{k} ; g^{0}\right)$, where $g^{0}$ is the effective matrix. Let $\widehat{Z}(\mathbf{k})$ and $\widehat{N}(\mathbf{k})$ be the operators defined by (4.3) and (4.10), respectively. If $\widehat{t}^{0}$ is defined by (4.17) and $\widehat{C}_{*}$ is the constant (4.21), then, for $|\mathbf{k}| \leq \widehat{t}^{0}$ and $\varepsilon>0$, we have

$$
\begin{aligned}
\left(\widehat{\mathcal{A}}(\mathbf{k})+\varepsilon^{2} I\right)^{-1}= & \widehat{R}^{0}(\mathbf{k}, \varepsilon)+\widehat{Z}(\mathbf{k}) \widehat{R}^{0}(\mathbf{k}, \varepsilon) \\
& +\widehat{R}^{0}(\mathbf{k}, \varepsilon) \widehat{Z}(\mathbf{k})^{*}-\widehat{R}^{0}(\mathbf{k}, \varepsilon) \widehat{N}(\mathbf{k}) \widehat{R}^{0}(\mathbf{k}, \varepsilon)+\widehat{J}(\mathbf{k})
\end{aligned}
$$

where $\widehat{R}^{0}(\mathbf{k}, \varepsilon)=\left(\widehat{\mathcal{A}}^{0}(\mathbf{k})+\varepsilon^{2} I\right)^{-1}$, and the remainder term $\widehat{J}(\mathbf{k})$ admits the estimate

$$
\|\widehat{J}(\mathbf{k})\|_{\mathfrak{H} \rightarrow \mathfrak{H}} \leq \widehat{C}_{*} .
$$


On the right-hand side of (4.22), the first term is called the leading term and the sum of the next three terms is called the "corrector", while the last term is called the remainder term. In what follows, we shall use this terminology systematically.

Applying Theorem 1.2 and recalling Proposition 4.5, we arrive at the following statement.

Theorem 4.7. Under the conditions of Theorem 4.6, let $\widehat{Z}(\boldsymbol{\theta})=0$, i.e., $\Lambda(\mathbf{x}) b(\boldsymbol{\theta})=0$, $\mathbf{x} \in \Omega$. Then, for $|\mathbf{k}| \leq \widehat{t}^{0}$ and $\varepsilon>0$, we have

$$
\left\|\left(\widehat{\mathcal{A}}(\mathbf{k})+\varepsilon^{2} I\right)^{-1}-\left(\widehat{\mathcal{A}}^{0}(\mathbf{k})+\varepsilon^{2} I\right)^{-1}\right\|_{\mathfrak{H} \rightarrow \mathfrak{H}} \leq 2 \widehat{C},
$$

where $\widehat{C}$ is the constant defined by (4.20). In particular, (4.23) is true under conditions (3.9), i.e., in the case where $g^{0}=\bar{g}$.

Remark 4.8. From (4.15)-(4.17), (4.20), and (4.21) we see that the constants $\widehat{t}^{0}, \widehat{C}$, and $\widehat{C}_{*}$ are determined in terms of the norms $\|g\|_{L_{\infty}},\left\|g^{-1}\right\|_{L_{\infty}}$, the constants $\alpha_{0}$ and $\alpha_{1}$ occurring in (2.2), and the parameter $r_{0}$ that depends on the lattice $\Gamma$.

4.5. Estimates for $\mathbf{k} \in \widetilde{\Omega} \backslash \mathcal{B}\left(\widehat{t}^{0}\right)$. In order to carry the statements of Theorems 4.6 and 4.7 over to all $\mathbf{k} \in \widetilde{\Omega}$, we need estimates for $\mathbf{k} \in \widetilde{\Omega} \backslash \mathcal{B}\left(\widehat{t}^{0}\right)$. Such estimates are trivial, because if $\mathbf{k} \in \widetilde{\Omega} \backslash \mathcal{B}\left(\widehat{t}^{0}\right)$, then all operators in (4.22) are uniformly bounded. However, we need to trace the constants in the estimates.

As was shown in [BSu2, (3.2.7)] (see also (2.20)), we have

$$
\left\|\left(\widehat{\mathcal{A}}(\mathbf{k})+\varepsilon^{2} I\right)^{-1}\right\|_{\mathfrak{H} \rightarrow \mathfrak{H}} \leq \widehat{c}_{*}^{-1}\left(\widehat{t}^{0}\right)^{-2}, \quad \mathbf{k} \in \widetilde{\Omega} \backslash \mathcal{B}\left(\widehat{t}^{0}\right),
$$

and the same estimate is true for $\left(\widehat{\mathcal{A}}^{0}(\mathbf{k})+\varepsilon^{2} I\right)^{-1}$. It follows that (4.22) remains true also for $\mathbf{k} \in \widetilde{\Omega} \backslash \mathcal{B}\left(\widehat{t}^{0}\right)$, with the estimate

$$
\|\widehat{J}(\mathbf{k})\| \leq \widehat{c}_{*}^{-1}\left(\widehat{t}^{0}\right)^{-2}\left(2+2\|\widehat{Z}(\mathbf{k})\|+\widehat{c}_{*}^{-1}\left(\widehat{t}^{0}\right)^{-2}\|\widehat{N}(\mathbf{k})\|\right), \quad \mathbf{k} \in \widetilde{\Omega} \backslash \mathcal{B}\left(\widehat{t}^{0}\right) .
$$

Since $\widehat{Z}(\mathbf{k})=t \widehat{Z}(\boldsymbol{\theta}), \widehat{N}(\mathbf{k})=t^{3} \widehat{N}(\boldsymbol{\theta})$ and $t=|\mathbf{k}| \leq r_{1}$, where $2 r_{1}$ is the diameter of $\widetilde{\Omega}$, we obtain

$$
\|\widehat{J}(\mathbf{k})\| \leq \widehat{c}_{*}^{-1}\left(\widehat{t}^{0}\right)^{-2}\left(2+2 r_{1}\|\widehat{Z}(\boldsymbol{\theta})\|+r_{1}^{3} \widehat{c}_{*}^{-1}\left(\widehat{t}^{0}\right)^{-2}\|\widehat{N}(\boldsymbol{\theta})\|\right), \quad \mathbf{k} \in \widetilde{\Omega} \backslash \mathcal{B}\left(\widehat{t}^{0}\right) .
$$

By (1.5), (1.14), and (4.14), we have

$$
\begin{aligned}
\|\widehat{Z}(\boldsymbol{\theta})\| & \leq(8 \widehat{\delta})^{-1 / 2}\left\|\widehat{X}_{1}(\boldsymbol{\theta})\right\| \leq(8 \widehat{\delta})^{-1 / 2} \alpha_{1}^{1 / 2}\|g\|_{L_{\infty}}^{1 / 2}, \\
\|\widehat{N}(\boldsymbol{\theta})\| & \leq(2 \widehat{\delta})^{-1 / 2}\left\|\widehat{X}_{1}(\boldsymbol{\theta})\right\|^{3} \leq(2 \widehat{\delta})^{-1 / 2} \alpha_{1}^{3 / 2}\|g\|_{L_{\infty}^{3 / 2}}^{3 / 2} .
\end{aligned}
$$

As a result, (4.25)-(4.27) imply that

$$
\begin{aligned}
\|\widehat{J}(\mathbf{k})\| & \leq \widehat{c}_{*}^{-1}\left(\widehat{t}^{0}\right)^{-2}\left(2+r_{1}(2 \widehat{\delta})^{-1 / 2} \alpha_{1}^{1 / 2}\|g\|_{L_{\infty}}^{1 / 2}+r_{1}^{3} \widehat{c}_{*}^{-1}\left(\widehat{t}^{0}\right)^{-2}(2 \widehat{\delta})^{-1 / 2} \alpha_{1}^{3 / 2}\|g\|_{L_{\infty}}^{3 / 2}\right) \\
& =: C_{\Delta}, \quad \mathbf{k} \in \widetilde{\Omega} \backslash \mathcal{B}\left(\widehat{t}^{0}\right) .
\end{aligned}
$$

Combining this with Theorem 4.6, we arrive at the following result.

Theorem 4.9. Let $\widehat{\mathcal{A}}(\mathbf{k})=\widehat{\mathcal{A}}(\mathbf{k} ; g)$, let $\widehat{\mathcal{A}}^{0}(\mathbf{k})=\widehat{\mathcal{A}}\left(\mathbf{k} ; g^{0}\right)$, and let $\widehat{Z}(\mathbf{k})$ and $\widehat{N}(\mathbf{k})$ be the operators defined by (4.3) and (4.10), respectively. Then (4.22) is valid for all $\mathbf{k} \in \widetilde{\Omega}$ and $\varepsilon>0$, and

$$
\|\widehat{J}(\mathbf{k})\|_{\mathfrak{H} \rightarrow \mathfrak{H}} \leq \max \left\{\widehat{C}_{*}, C_{\Delta}\right\}=: \widehat{\mathcal{C}}_{1} .
$$

Here $\widehat{C}_{*}$ and $C_{\Delta}$ are defined by (4.21) and (4.28).

Moreover, combining Theorem 4.7 with estimate (4.24) and the same estimate for $\left(\widehat{\mathcal{A}}^{0}(\mathbf{k})+\varepsilon^{2} I\right)^{-1}$, we directly obtain the following theorem. 
Theorem 4.10. Let $\widehat{\mathcal{A}}(\mathbf{k})=\widehat{\mathcal{A}}(\mathbf{k} ; g)$, and let $\widehat{\mathcal{A}}^{0}(\mathbf{k})=\widehat{\mathcal{A}}\left(\mathbf{k} ; g^{0}\right)$. Suppose that $\widehat{Z}(\boldsymbol{\theta})=0$, i.e., $\Lambda(\mathbf{x}) b(\boldsymbol{\theta})=0, \mathbf{x} \in \Omega$. Then, for all $\mathbf{k} \in \widetilde{\Omega}$ and $\varepsilon>0$, we have

$$
\left\|\left(\widehat{\mathcal{A}}(\mathbf{k})+\varepsilon^{2} I\right)^{-1}-\left(\widehat{\mathcal{A}}^{0}(\mathbf{k})+\varepsilon^{2} I\right)^{-1}\right\|_{\mathfrak{H} \rightarrow \mathfrak{H}} \leq 2 \max \left\{\widehat{C}, \widehat{c}_{*}^{-1}\left(\widehat{t}^{0}\right)^{-2}\right\}=: \widehat{\mathcal{C}}_{2} .
$$

Here $\widehat{c}_{*}$ and $\widehat{t}^{0}$ are defined by (4.15) and (4.17), and $\widehat{C}$ is defined by (4.20). In particular, (4.30) is true under conditions (3.9) (i.e., in the case where $g^{0}=\bar{g}$ ).

Remark 4.11. From Remark 4.8, and also from (4.15)-(4.17) and (4.28)-(4.30), we see that the constants $\widehat{\mathcal{C}_{1}}$ and $\widehat{\mathcal{C}_{2}}$ depend only on $\|g\|_{L_{\infty}},\left\|g^{-1}\right\|_{L_{\infty}}, \alpha_{0}, \alpha_{1}$, and on the parameters of the lattice.

\section{$\S 5$. Approximation of the generalized Resolvent $\left(\widehat{\mathcal{A}}(\mathbf{k})+\varepsilon^{2} Q\right)^{-1}$}

5.1. Now we consider the operator $\mathcal{A}$ of the general form:

$$
\mathcal{A}=\mathcal{A}(g, f)=f(\mathbf{x})^{*} b(\mathbf{D})^{*} g(\mathbf{x}) b(\mathbf{D}) f(\mathbf{x})=f^{*} \widehat{\mathcal{A}}(g) f .
$$

Let $\mathcal{A}(\mathbf{k})$ and $\widehat{\mathcal{A}}(\mathbf{k})$ be the operator families occurring in the direct integral decompositions for $\mathcal{A}$ and $\widehat{\mathcal{A}}$. Then $\mathcal{A}(\mathbf{k})=f^{*} \widehat{\mathcal{A}}(\mathbf{k}) f$.

We are under the assumptions of Subsection 1.6. Now $\mathfrak{H}=\widehat{\mathfrak{H}}=L_{2}\left(\Omega ; \mathbb{C}^{n}\right)$, the role of the $A(t)$ is played by $A(t, \boldsymbol{\theta})=\mathcal{A}(\mathbf{k})$, and the role of $\widehat{A}(t)$ is played by $\widehat{A}(t, \boldsymbol{\theta})=\widehat{\mathcal{A}}(\mathbf{k})$. The isomorphism $M$ is multiplication by the matrix-valued function $f(\mathbf{x})$. Next,

$$
\begin{aligned}
X_{0} & =h(\mathbf{x}) b(\mathbf{D}) f(\mathbf{x}), & \widehat{X}_{0} & =h(\mathbf{x}) b(\mathbf{D}), \\
X_{1}(\boldsymbol{\theta}) & =h(\mathbf{x}) b(\boldsymbol{\theta}) f(\mathbf{x}), & \widehat{X}_{1}(\boldsymbol{\theta}) & =h(\mathbf{x}) b(\boldsymbol{\theta}) .
\end{aligned}
$$

The kernel $\widehat{\mathfrak{N}}$ consists of constant vectors (see (3.1)), and the kernel $\mathfrak{N}$ is defined by (2.13). By (1.30), $Q$ is multiplication by the positive matrix-valued function

$$
Q(\mathbf{x}):=\left(f(\mathbf{x}) f(\mathbf{x})^{*}\right)^{-1} .
$$

The block $Q_{\widehat{\mathfrak{N}}}$ of the operator $Q$ in the kernel $\widehat{\mathfrak{N}}$ is multiplication by the constant matrix $\bar{Q}$ (the mean value of $Q$ ):

$$
Q_{\widehat{\mathfrak{N}}}=\bar{Q}=\left(\underline{f f^{*}}\right)^{-1}
$$

(cf. Subsection 3.4).

5.2. The operator $\widehat{Z}_{Q}(\boldsymbol{\theta})$. As in Subsection 1.7, we introduce the operator $\widehat{Z}_{Q}(\boldsymbol{\theta})$, acting in $\mathfrak{H}$, that takes an element $\widehat{\mathbf{u}} \in \mathfrak{H}$ to the solution $\mathbf{w}^{(Q)} \in \widetilde{H}^{1}\left(\Omega ; \mathbb{C}^{n}\right)$ of the problem

$$
b(\mathbf{D})^{*} g(\mathbf{x})\left(b(\mathbf{D}) \mathbf{w}^{(Q)}(\mathbf{x})+b(\boldsymbol{\theta}) \mathbf{c}\right)=0, \quad \int_{\Omega} Q(\mathbf{x}) \mathbf{w}^{(Q)}(\mathbf{x}) d \mathbf{x}=0, \quad \mathbf{c}=\widehat{P} \widehat{\mathbf{u}} .
$$

Recall that $\widehat{P}$ is the operator of averaging over the cell (see (3.2)).

By analogy with the operator $\Lambda$ (see Subsection 3.1), we introduce the operator $\Lambda_{Q}$ : $\mathbb{C}^{m} \rightarrow \mathfrak{H}$ that takes a vector $\mathbf{C} \in \mathbb{C}^{m}$ to the periodic solution $\mathbf{v}_{\mathbf{C}}^{(Q)} \in \widetilde{H}^{1}\left(\Omega ; \mathbb{C}^{n}\right)$ of the problem

$$
b(\mathbf{D})^{*} g(\mathbf{x})\left(b(\mathbf{D}) \mathbf{v}_{\mathbf{C}}^{(Q)}(\mathbf{x})+\mathbf{C}\right)=0, \quad \int_{\Omega} Q(\mathbf{x}) \mathbf{v}_{\mathbf{C}}^{(Q)}(\mathbf{x}) d \mathbf{x}=0 .
$$

The solution $\mathbf{v}_{\mathbf{e}_{j}}^{(Q)}$ of problem (5.3) with $\mathbf{C}=\mathbf{e}_{j}$ is denoted by $\mathbf{v}_{j}^{(Q)}, j=1, \ldots, m$. Let $\Lambda_{Q}(\mathbf{x})$ be the periodic $(n \times m)$-matrix-valued function with the columns $\mathbf{v}_{j}^{(Q)}(\mathbf{x})$, 
$j=1, \ldots, m$. Clearly, the matrix $\Lambda_{Q}(\mathbf{x})$ differs from $\Lambda(\mathbf{x})$ (introduced in Subsection 3.1) by a constant summand:

$$
\Lambda_{Q}(\mathbf{x})=\Lambda(\mathbf{x})+\Lambda_{Q}^{0}, \quad \Lambda_{Q}^{0}=-(\bar{Q})^{-1}(\overline{Q \Lambda}) .
$$

The operator $\Lambda_{Q}$ acts as multiplication by the matrix $\Lambda_{Q}(\mathbf{x})$. We have (cf. (4.2))

$$
\widehat{Z}_{Q}(\boldsymbol{\theta})=\Lambda_{Q}(\mathbf{x}) b(\boldsymbol{\theta}) \widehat{P} .
$$

Clearly, the relations $\Lambda=0$ and $\Lambda_{Q}=0$ are equivalent. Therefore, Remark 4.1 implies the following.

Remark 5.1. If $g^{0}=\bar{g}$ (i.e., under conditions (3.9)), we have $\Lambda_{Q}=0$ and $\widehat{Z}_{Q}(\boldsymbol{\theta})=0$. In particular, this is true if $g$ is a constant matrix.

We put $\widehat{Z}_{Q}(\mathbf{k})=t \widehat{Z}_{Q}(\boldsymbol{\theta})$. Then

$$
\widehat{Z}_{Q}(\mathbf{k})=\Lambda_{Q}(\mathbf{x}) b(\mathbf{k}) \widehat{P}, \quad \mathbf{k} \in \mathbb{R}^{d} .
$$

By (1.38), the operator $\widehat{Z}_{Q}(\boldsymbol{\theta})$ admits the estimate

$$
\left\|\widehat{Z}_{Q}(\boldsymbol{\theta})\right\| \leq(8 \delta)^{-1 / 2}\left\|X_{1}(\boldsymbol{\theta})\right\|\|f\|_{L_{\infty}}\left\|f^{-1}\right\|_{L_{\infty}} .
$$

Combining the obvious estimate

$$
\left\|X_{1}(\boldsymbol{\theta})\right\| \leq \alpha_{1}^{1 / 2}\|h\|_{L_{\infty}}\|f\|_{L_{\infty}}
$$

with (2.18) and (5.7), we obtain

$$
\begin{aligned}
\left\|\widehat{Z}_{Q}(\boldsymbol{\theta})\right\| & \leq(8 \delta)^{-1 / 2} \alpha_{1}^{1 / 2}\|h\|_{L_{\infty}}\|f\|_{L_{\infty}}^{2}\left\|f^{-1}\right\|_{L_{\infty}} \\
& =2^{-1 / 2} r_{0}^{-1} \alpha_{1}^{1 / 2} \alpha_{0}^{-1 / 2}\|g\|_{L_{\infty}}^{1 / 2}\left\|g^{-1}\right\|_{L_{\infty}}^{1 / 2}\|f\|_{L_{\infty}}^{2}\left\|f^{-1}\right\|_{L_{\infty}}^{2}=: C_{1}^{(Q)} .
\end{aligned}
$$

5.3. The operator $\widehat{N}_{Q}(\boldsymbol{\theta})$. By (1.37), we have

$$
\widehat{N}_{Q}(\boldsymbol{\theta})=\left(\widehat{Z}_{Q}(\boldsymbol{\theta})\right)^{*} \widehat{X}_{1}(\boldsymbol{\theta})^{*} \widehat{R}(\boldsymbol{\theta}) \widehat{P}+(\widehat{R}(\boldsymbol{\theta}) \widehat{P})^{*} \widehat{X}_{1}(\boldsymbol{\theta}) \widehat{Z}_{Q}(\boldsymbol{\theta}) .
$$

Combining this with (4.6) and (5.5), we see that

$$
\widehat{N}_{Q}(\boldsymbol{\theta})=\widehat{P} b(\boldsymbol{\theta})^{*}\left(\left(\Lambda_{Q}(\mathbf{x})\right)^{*} b(\boldsymbol{\theta})^{*} \widetilde{g}(\mathbf{x})+\widetilde{g}(\mathbf{x})^{*} b(\boldsymbol{\theta}) \Lambda_{Q}(\mathbf{x})\right) b(\boldsymbol{\theta}) \widehat{P} .
$$

We introduce the notation

$$
L_{Q}(\boldsymbol{\theta}):=|\Omega|^{-1} \int_{\Omega}\left(\left(\Lambda_{Q}(\mathbf{x})\right)^{*} b(\boldsymbol{\theta})^{*} \widetilde{g}(\mathbf{x})+\widetilde{g}(\mathbf{x})^{*} b(\boldsymbol{\theta}) \Lambda_{Q}(\mathbf{x})\right) d \mathbf{x} .
$$

Substituting (5.4) into (5.11) and using (3.6) and (4.8), we obtain

$$
L_{Q}(\boldsymbol{\theta})=L(\boldsymbol{\theta})+L_{Q}^{0}(\boldsymbol{\theta}), \quad L_{Q}^{0}(\boldsymbol{\theta})=\left(\Lambda_{Q}^{0}\right)^{*} b(\boldsymbol{\theta})^{*} g^{0}+g^{0} b(\boldsymbol{\theta}) \Lambda_{Q}^{0} .
$$

From (5.10) and (5.11) it follows that

$$
\widehat{N}_{Q}(\boldsymbol{\theta})=b(\boldsymbol{\theta})^{*} L_{Q}(\boldsymbol{\theta}) b(\boldsymbol{\theta}) \widehat{P} .
$$

We put $t^{3} \widehat{N}_{Q}(\boldsymbol{\theta})=: \widehat{N}_{Q}(\mathbf{k})$. Then

$$
\widehat{N}_{Q}(\mathbf{k})=b(\mathbf{k})^{*} L_{Q}(\mathbf{k}) b(\mathbf{k}) \widehat{P}, \quad \mathbf{k} \in \mathbb{R}^{d} .
$$

By (1.39), the operator $\widehat{N}_{Q}(\boldsymbol{\theta})$ admits the estimate

$$
\left\|\widehat{N}_{Q}(\boldsymbol{\theta})\right\| \leq(2 \delta)^{-1 / 2}\left\|X_{1}(\boldsymbol{\theta})\right\|\|f\|_{L_{\infty}}\left\|f^{-1}\right\|_{L_{\infty}}\left\|\widehat{X}_{1}(\boldsymbol{\theta})\right\|^{2} .
$$

Combining this with (2.18), (4.14), and (5.8), we arrive at the inequality

$$
\begin{aligned}
\left\|\widehat{N}_{Q}(\boldsymbol{\theta})\right\| & \leq(2 \delta)^{-1 / 2} \alpha_{1}^{3 / 2}\|g\|_{L_{\infty}}^{3 / 2}\|f\|_{L_{\infty}}^{2}\left\|f^{-1}\right\|_{L_{\infty}} \\
& =2^{1 / 2} r_{0}^{-1} \alpha_{1}^{3 / 2} \alpha_{0}^{-1 / 2}\|g\|_{L_{\infty}}^{3 / 2}\left\|g^{-1}\right\|_{L_{\infty}}^{1 / 2}\|f\|_{L_{\infty}}^{2}\left\|f^{-1}\right\|_{L_{\infty}}^{2}=: C_{2}^{(Q)} .
\end{aligned}
$$


5.4. If the matrices $b(\boldsymbol{\theta}), g(\mathbf{x})$, and $Q(\mathbf{x})$ have real entries, the matrix $\widetilde{g}(\mathbf{x})$ also has real entries, while $\Lambda_{Q}(\mathbf{x})$ has purely imaginary entries. Then $L_{Q}(\boldsymbol{\theta})$ and $b(\boldsymbol{\theta})^{*} L_{Q}(\boldsymbol{\theta}) b(\boldsymbol{\theta})$ are Hermitian matrices with purely imaginary entries. Therefore, $\left(\widehat{N}_{Q}(\boldsymbol{\theta}) \mathbf{q}, \mathbf{q}\right)_{\mathfrak{H}}=0$ for any real vector $\mathbf{q} \in \widehat{\mathfrak{N}}$. If the analytic branches of the eigenvalues $\lambda_{l}(t, \boldsymbol{\theta})$ and the branches of the eigenvectors $\varphi_{l}(t, \boldsymbol{\theta})$ of $A(t, \boldsymbol{\theta})$ (see Subsection 3.4) can be chosen so that the vectors $\widehat{\phi}_{l}(\boldsymbol{\theta})=f \omega_{l}(\boldsymbol{\theta}) \in \widehat{\mathfrak{N}}$ are real, then, by $(1.47)$,

$$
\mu_{l}(\boldsymbol{\theta})=\left(\widehat{N}_{Q}(\boldsymbol{\theta}) \widehat{\phi}_{l}(\boldsymbol{\theta}), \widehat{\phi}_{l}(\boldsymbol{\theta})\right)_{\mathfrak{H}}=0, \quad l=1, \ldots, n,
$$

whence

$$
\lambda_{l}(t, \boldsymbol{\theta})=\gamma_{l}(\boldsymbol{\theta}) t^{2}+O\left(t^{4}\right), \quad l=1, \ldots, n .
$$

We have proved the following statement.

Proposition 5.2. Let $b(\boldsymbol{\theta}), g(\mathbf{x})$, and $Q(\mathbf{x})$ be matrices with real entries. Suppose that, in the expansion (1.8) for the analytic branches of the eigenvectors of $A(t, \theta)$, the elements $\omega_{l}(\boldsymbol{\theta}), l=1, \ldots, n$, can be chosen so that the vectors $\widehat{\phi}_{l}(\boldsymbol{\theta})=f \omega_{l}(\boldsymbol{\theta})$ are real. Then in (1.7) we have $\mu_{l}(\boldsymbol{\theta})=0, l=1, \ldots, n$, and relations (5.17) are valid. Consequently, $\widehat{N}_{0, Q}(\boldsymbol{\theta})=0($ see $(1.41))$.

Corollary 5.3. Suppose that the matrices $b(\boldsymbol{\theta}), g(\mathbf{x})$, and $Q(\mathbf{x})$ have real entries. If the spectrum of the generalized spectral problem (3.11) is simple, then (5.17) is true.

Proof. Since the vectors $\widehat{\phi}_{l}(\boldsymbol{\theta})=f \omega_{l}(\boldsymbol{\theta})$ are eigenvectors of problem (3.11), they are defined uniquely up to phase factors and can be chosen to be real.

Corollary 5.4. Let $g(\mathbf{x})$ be a periodic positive definite $(d \times d)$-matrix-valued function with real entries. Suppose that $g, g^{-1} \in L_{\infty}$. If $f(\mathbf{x})$ is a periodic function such that $f, f^{-1} \in L_{\infty}$, then for the operator $\mathcal{A}(g, f)=f(\mathbf{x})^{*} \mathbf{D}^{*} g(\mathbf{x}) \mathbf{D} f(\mathbf{x})$ we have $\mu(\boldsymbol{\theta})=0$ and $\widehat{N}_{Q}(\theta)=0$.

Indeed, in this case $n=1$ and, by (5.16), the only matrix element of the operator $\widehat{N}_{Q}(\theta)$ is equal to zero.

We note that (see $\S 11$ below), under the condition $\inf \operatorname{spec} \mathcal{H}=0$, the periodic Schrödinger operator $\mathcal{H}=\mathbf{D}^{*} \mathfrak{g}(\mathbf{x}) \mathbf{D}+p(\mathbf{x})$ can be written in the form $f(\mathbf{x})^{*} \mathbf{D}^{*} g(\mathbf{x}) \mathbf{D} f(\mathbf{x})$ with appropriate $g$ and $f$.

Recall that the conditions $\widehat{Z}(\boldsymbol{\theta})=0$ and $\widehat{Z}_{Q}(\boldsymbol{\theta})=0$ are equivalent (see Remark 1.6). Hence, Proposition 4.5(1) implies that under conditions (3.9) (i.e., for $g^{0}=\bar{g}$ ) we have $\widehat{Z}_{Q}(\boldsymbol{\theta})=0$, whence also $\widehat{N}_{Q}(\boldsymbol{\theta})=0$. There is no direct analog of Proposition 4.5(2) for $\widehat{N}_{Q}(\boldsymbol{\theta})$. This is explained by the fact that, in general, the mean value of $\Lambda_{Q}(\mathbf{x})$ over the cell is nonzero (unlike the mean value of $\Lambda$ ).

5.5. Approximation of the generalized resolvent $\left(\widehat{\mathcal{A}}(\mathbf{k})+\varepsilon^{2} Q\right)^{-1}$ for $|\mathbf{k}| \leq t^{0}$. Let $g^{0}$ be the effective matrix, and let $\widehat{\mathcal{A}}^{0}=\widehat{\mathcal{A}}\left(g^{0}\right)$ be the effective operator for $\widehat{\mathcal{A}}(g)$. Then the families $\widehat{A}(t, \boldsymbol{\theta})=\widehat{A}(t, \boldsymbol{\theta} ; g)$ and $\widehat{A}^{0}(t, \boldsymbol{\theta})=\widehat{A}\left(t, \boldsymbol{\theta} ; g^{0}\right)$ are threshold equivalent. Our goal is to apply Theorem 1.4. The role of $\widehat{A}(t)$ is played by $\widehat{A}(t, \boldsymbol{\theta})$, and the role of $\widehat{A}_{+}(t)$ is played by $\widehat{A}^{0}(t, \boldsymbol{\theta})$. The isomorphism $M$ is multiplication by $f(\mathbf{x})$, and the family $A(t)$ is realized as $A(t, \boldsymbol{\theta})=f^{*} \widehat{A}(t, \boldsymbol{\theta}) f$. The operator $Q$ is multiplication by the matrix (5.1). The block $Q_{\widehat{\mathfrak{N}}}($ see $(5.2))$ is multiplication by the constant matrix $\bar{Q}=\left(\underline{f f^{*}}\right)^{-1}$. We put

$$
Q_{+}:=\bar{Q}, \quad M_{+}:=(\bar{Q})^{-1 / 2}=\left(\underline{f f^{*}}\right)^{1 / 2}=: f_{0} .
$$


Clearly, the blocks of $Q$ and $Q_{+}$in $\widehat{\mathfrak{N}}$ coincide. The operator $A_{+}(t)$ is realized as $A_{+}(t, \boldsymbol{\theta})=f_{0} \widehat{A}^{0}(t, \boldsymbol{\theta}) f_{0}$. Next, by Remark 5.1, $\widehat{Z}_{Q_{+}}^{0}(\boldsymbol{\theta})=0$ (here $\widehat{Z}_{Q_{+}}^{0}(\boldsymbol{\theta})$ is the counterpart of the operator $\widehat{Z}_{Q}$ for the family $\widehat{A}^{0}(t, \theta)$ and the operator $\left.Q_{+}\right)$. We have checked all the conditions of Theorem 1.4.

Let us trace the constants. For the family $A(t, \boldsymbol{\theta})$, the constants $c_{*}, \delta$ and $t^{0}$ are defined by (2.17)-(2.19). Since, obviously,

$$
\left\|f_{0}\right\|_{L_{\infty}} \leq\|f\|_{L_{\infty}}, \quad\left\|f_{0}^{-1}\right\|_{L_{\infty}} \leq\left\|f^{-1}\right\|_{L_{\infty}},
$$

and (4.18) is satisfied, the similar constants $c_{*}^{(+)}, \delta_{+}$and $t_{+}^{0}$ for the family $A_{+}(t, \theta)$ can only be greater than the constants for $A(t, \boldsymbol{\theta})$.

Next, for the operator $A(t, \theta)$ the role of the constant (1.25) is played by

$$
C(\boldsymbol{\theta})=\delta^{-1}\left(\beta_{1} c_{*}^{-1}\left\|X_{1}(\boldsymbol{\theta})\right\|^{2}+\beta_{2} c_{*}^{-2}\left\|X_{1}(\boldsymbol{\theta})\right\|^{4}+\beta_{3} c_{*}^{-3}\left\|X_{1}(\boldsymbol{\theta})\right\|^{6}+3^{-1}\right) .
$$

It is convenient (see (5.8)) to use the constant

$$
\begin{aligned}
\mathcal{C}=\delta^{-1}\left(\beta_{1} c_{*}^{-1} \alpha_{1}\|g\|_{L_{\infty}}\|f\|_{L_{\infty}}^{2}\right. & \\
& \left.+\beta_{2} c_{*}^{-2} \alpha_{1}^{2}\|g\|_{L_{\infty}}^{2}\|f\|_{L_{\infty}}^{4}+\beta_{3} c_{*}^{-3} \alpha_{1}^{3}\|g\|_{L_{\infty}}^{3}\|f\|_{L_{\infty}}^{6}+3^{-1}\right)
\end{aligned}
$$

instead of $C(\boldsymbol{\theta})$; the constant (5.18) does not depend on $\boldsymbol{\theta}$.

The constant $C_{+}(\boldsymbol{\theta})$, which plays the role of the constant (1.26) for the operator $A_{+}(t, \boldsymbol{\theta})$, can also be roughened. The new constant may only become smaller than $\mathcal{C}$. Finally, we can use the constant

$$
\check{\mathcal{C}}=\mathcal{C}\|f\|_{L_{\infty}}^{2}\left(2+2^{-1 / 2}\|f\|_{L_{\infty}}\left\|f^{-1}\right\|_{L_{\infty}}\left(1+2 \alpha_{0}^{-1} \alpha_{1}\|g\|_{L_{\infty}}\left\|g^{-1}\right\|_{L_{\infty}}\right)\right)
$$

instead of $\breve{C}(\boldsymbol{\theta})$, which now realizes (1.49). We have used (4.14) and (4.15).

Applying Theorem 1.4, we arrive at the following result.

Theorem 5.5. Let $\widehat{\mathcal{A}}(\mathbf{k})=\widehat{\mathcal{A}}(\mathbf{k} ; g)$, and let $\widehat{\mathcal{A}}^{0}(\mathbf{k})=\widehat{\mathcal{A}}\left(\mathbf{k} ; g^{0}\right)$, where $g^{0}$ is the effective matrix. Let $Q(\mathbf{x})$ be a periodic positive definite $(n \times n)$-matrix-valued function such that $Q, Q^{-1} \in L_{\infty}$. Suppose that $Q(\mathbf{x})$ is factorized as $Q(\mathbf{x})^{-1}=f(\mathbf{x}) f(\mathbf{x})^{*}$. Let $\bar{Q}=$ $|\Omega|^{-1} \int_{\Omega} Q(\mathbf{x}) d \mathbf{x}$. Suppose that the operators $\widehat{Z}_{Q}(\mathbf{k})$ and $\widehat{N}_{Q}(\mathbf{k})$ are defined by (5.6) and (5.14), respectively. Let $t^{0}$ be the number defined by (2.19), and let $\breve{\mathcal{C}}$ be defined by (5.18), (5.19). Then, for $|\mathbf{k}| \leq t^{0}$ and $\varepsilon>0$, we have

$$
\begin{aligned}
\left(\widehat{\mathcal{A}}(\mathbf{k})+\varepsilon^{2} Q\right)^{-1}= & \widehat{R}_{Q}^{0}(\mathbf{k}, \varepsilon)+\widehat{Z}_{Q}(\mathbf{k}) \widehat{R}_{Q}^{0}(\mathbf{k}, \varepsilon) \\
& +\widehat{R}_{Q}^{0}(\mathbf{k}, \varepsilon) \widehat{Z}_{Q}(\mathbf{k})^{*}-\widehat{R}_{Q}^{0}(\mathbf{k}, \varepsilon) \widehat{N}_{Q}(\mathbf{k}) \widehat{R}_{Q}^{0}(\mathbf{k}, \varepsilon)+\check{J}(\mathbf{k}),
\end{aligned}
$$

where $\widehat{R}_{Q}^{0}(\mathbf{k}, \varepsilon)=\left(\widehat{\mathcal{A}}^{0}(\mathbf{k})+\varepsilon^{2} \bar{Q}\right)^{-1}$, and $\check{J}(\mathbf{k})$ admits the estimate

$$
\|\check{J}(\mathbf{k})\|_{\mathfrak{H} \rightarrow \mathfrak{H}} \leq \check{\mathcal{C}} .
$$

Similarly, applying Theorem 1.5 and using Remark 1.6 and Proposition 4.5(1), we obtain the following statement.

Theorem 5.6. Under the assumptions of Theorem 5.5, suppose that $\Lambda(\mathbf{x}) b(\boldsymbol{\theta})=0$, $\mathbf{x} \in \Omega$. Then, for $|\mathbf{k}| \leq t^{0}$ and $\varepsilon>0$, we have

$$
\left\|\left(\widehat{\mathcal{A}}(\mathbf{k})+\varepsilon^{2} Q\right)^{-1}-\left(\widehat{\mathcal{A}}^{0}(\mathbf{k})+\varepsilon^{2} \bar{Q}\right)^{-1}\right\|_{\mathfrak{H} \rightarrow \mathfrak{H}} \leq 2 \mathcal{C}\left\|Q^{-1}\right\|_{L_{\infty}},
$$

where $\mathcal{C}$ is the constant defined by (5.18). In particular, (5.21) is true under conditions (3.9), i.e., in the case where $g^{0}=\bar{g}$.

Remark 5.7. From (2.17)-(2.19), (5.18), and (5.19) we see that the constants $t^{0}, \mathcal{C}$, and $\check{\mathcal{C}}$ depend only on the norms $\|g\|_{L_{\infty}},\left\|g^{-1}\right\|_{L_{\infty}},\|f\|_{L_{\infty}},\left\|f^{-1}\right\|_{L_{\infty}}$, the parameters $\alpha_{0}$ and $\alpha_{1}$ in (2.2), and the parameter $r_{0}$ determined by the lattice $\Gamma$. 
5.6. Estimates for $\mathbf{k} \in \widetilde{\Omega} \backslash \mathcal{B}\left(t^{0}\right)$. Estimates for $\mathbf{k} \in \widetilde{\Omega} \backslash \mathcal{B}\left(t^{0}\right)$ are trivial (cf. Subsection 4.5). Indeed, for $\mathbf{k} \in \widetilde{\Omega} \backslash \mathcal{B}\left(t^{0}\right)$ all operators in (5.20) are uniformly bounded. We must only control the constants. As was shown in BSu2, Subsection 3.3.1], we have

$$
\left\|\left(\widehat{\mathcal{A}}(\mathbf{k})+\varepsilon^{2} Q\right)^{-1}\right\|_{\mathfrak{H} \rightarrow \mathfrak{H}} \leq\left\|Q^{-1}\right\|_{L_{\infty}} c_{*}^{-1}\left(t^{0}\right)^{-2}, \quad \mathbf{k} \in \widetilde{\Omega} \backslash \mathcal{B}\left(t^{0}\right),
$$

and the same estimate is true for $\left(\widehat{\mathcal{A}}^{0}(\mathbf{k})+\varepsilon^{2} \bar{Q}\right)^{-1}$. It follows that for $\mathbf{k} \in \widetilde{\Omega} \backslash \mathcal{B}\left(t^{0}\right)$, (5.20) remains valid with the estimate

$$
\begin{array}{r}
\|\check{J}(\mathbf{k})\| \leq\left\|Q^{-1}\right\|_{L_{\infty}} c_{*}^{-1}\left(t^{0}\right)^{-2}\left(2+2\left\|\widehat{Z}_{Q}(\mathbf{k})\right\|+\left\|Q^{-1}\right\|_{L_{\infty}} c_{*}^{-1}\left(t^{0}\right)^{-2}\left\|\widehat{N}_{Q}(\mathbf{k})\right\|\right), \\
\mathbf{k} \in \widetilde{\Omega} \backslash \mathcal{B}\left(t^{0}\right) .
\end{array}
$$

Hence (cf. (4.25)),

$$
\begin{aligned}
\|\breve{J}(\mathbf{k})\| \leq & \left\|Q^{-1}\right\|_{L_{\infty}} c_{*}^{-1}\left(t^{0}\right)^{-2} \\
& \times\left(2+2 r_{1}\left\|\widehat{Z}_{Q}(\boldsymbol{\theta})\right\|+r_{1}^{3}\left\|Q^{-1}\right\|_{L_{\infty}} c_{*}^{-1}\left(t^{0}\right)^{-2}\left\|\widehat{N}_{Q}(\boldsymbol{\theta})\right\|\right), \quad \mathbf{k} \in \widetilde{\Omega} \backslash \mathcal{B}\left(t^{0}\right) .
\end{aligned}
$$

Finally, by (5.9) and (5.15), we have

$$
\begin{aligned}
\|\check{J}(\mathbf{k})\| \leq & \left\|Q^{-1}\right\|_{L_{\infty}} c_{*}^{-1}\left(t^{0}\right)^{-2} \\
& \times\left(2+2 r_{1} C_{1}^{(Q)}+r_{1}^{3}\left\|Q^{-1}\right\|_{L_{\infty}} c_{*}^{-1}\left(t^{0}\right)^{-2} C_{2}^{(Q)}\right) \\
= & : \check{C}_{\Delta}, \quad \mathbf{k} \in \widetilde{\Omega} \backslash \mathcal{B}\left(t^{0}\right) .
\end{aligned}
$$

Combining this with Theorem 5.5 leads to the following statement.

Theorem 5.8. Under the assumptions of Theorem 5.5, the representation (5.20) is valid for all $\mathbf{k} \in \widetilde{\Omega}$ and $\varepsilon>0$, and

$$
\|\check{J}(\mathbf{k})\|_{\mathfrak{H} \rightarrow \mathfrak{H}} \leq \max \left\{\check{\mathcal{C}}, \check{C}_{\Delta}\right\}=: \mathcal{C}_{1} .
$$

Here $\check{\mathcal{C}}$ is defined by (5.18) and (5.19), and $\check{C}_{\Delta}$ is defined in accordance with (5.9), (5.15), and (5.23).

The next statement is deduced from Theorem 5.6, estimate (5.22), and a similar estimate for $\left(\widehat{\mathcal{A}}^{0}(\mathbf{k})+\varepsilon^{2} \bar{Q}\right)^{-1}$.

Theorem 5.9. Under the assumptions of Theorem 5.6 , for all $\mathbf{k} \in \widetilde{\Omega}$ and $\varepsilon>0$ we have

$$
\begin{array}{r}
\left\|\left(\widehat{\mathcal{A}}(\mathbf{k})+\varepsilon^{2} Q\right)^{-1}-\left(\widehat{\mathcal{A}}^{0}(\mathbf{k})+\varepsilon^{2} \bar{Q}\right)^{-1}\right\|_{\mathfrak{H} \rightarrow \mathfrak{H}} \\
\leq 2\left\|Q^{-1}\right\|_{L_{\infty}} \max \left\{\mathcal{C}, c_{*}^{-1}\left(t^{0}\right)^{-2}\right\}=: \mathcal{C}_{2} .
\end{array}
$$

Here, $c_{*}$ and $t^{0}$ are defined by (2.17) and (2.19), and $\mathcal{C}$ is defined by (5.18). In particular, (5.25) is true under conditions (3.9) (i.e., in the case where $g^{0}=\bar{g}$ ).

Remark 5.10. Remark 5.7 and relations (2.17), (2.19), (5.9), (5.15), and (5.23)-(5.25) show that the constants $\mathcal{C}_{1}$ and $\mathcal{C}_{2}$ depend only on $\|g\|_{L_{\infty}},\left\|g^{-1}\right\|_{L_{\infty}},\|f\|_{L_{\infty}},\left\|f^{-1}\right\|_{L_{\infty}}$, $\alpha_{0}, \alpha_{1}$, and the parameters of the lattice.

By using the relation

$$
\left(\mathcal{A}(\mathbf{k})+\varepsilon^{2} I\right)^{-1}=f^{-1}\left(\widehat{\mathcal{A}}(\mathbf{k})+\varepsilon^{2} Q\right)^{-1}\left(f^{*}\right)^{-1}
$$

(cf. (1.48)), analogs of Theorems 5.5, 5.6, 5.8, and 5.9 can be proved for the resolvent $\left(\mathcal{A}(\mathbf{k})+\varepsilon^{2} I\right)^{-1}$, simply by multiplying the operators in $(5.20),(5.21)$, and $(5.25)$ by $f^{-1}$ from the left and by $\left(f^{*}\right)^{-1}$ from the right. In this case the constants in the estimates for the remainder terms must be multiplied by $\left\|f^{-1}\right\|_{L_{\infty}}^{2}$. (Cf. Theorems 1.7 and 1.8.) We do not dwell on the detailed formulations. 


\section{§6. Approximation of the Resolvent $\left(\widehat{\mathcal{A}}+\varepsilon^{2} I\right)^{-1}$}

6.1. The resolvent in $L_{2}\left(\mathbb{R}^{d}\right)$. The operators $\widehat{\mathcal{Z}}$ and $\widehat{\mathcal{N}}$. Now we consider the operator $\widehat{\mathcal{A}}=\widehat{\mathcal{A}}(g)=b(\mathbf{D})^{*} g(\mathbf{x}) b(\mathbf{D})$, acting in the space $\mathfrak{G}:=L_{2}\left(\mathbb{R}^{d} ; \mathbb{C}^{n}\right)$. Let $g^{0}$ be the effective matrix, let $\widehat{\mathcal{A}}^{0}=\widehat{\mathcal{A}}\left(g^{0}\right)$ be the effective operator, and let $\mathcal{V}: \mathfrak{G} \rightarrow \mathcal{K}$ be the (unitary) Gelfand transformation defined in Subsection 2.3. Decomposing $\widehat{\mathcal{A}}$ as in (2.16), we obtain the following representation for the resolvent $\left(\widehat{\mathcal{A}}+\varepsilon^{2} I\right)^{-1}$ :

$$
\left(\widehat{\mathcal{A}}+\varepsilon^{2} I\right)^{-1}=\mathcal{V}^{-1}\left(\int_{\widetilde{\Omega}} \oplus\left(\widehat{\mathcal{A}}(\mathbf{k})+\varepsilon^{2} I\right)^{-1} d \mathbf{k}\right) \mathcal{V}
$$

A similar representation is valid also for $\left(\widehat{\mathcal{A}}^{0}+\varepsilon^{2} I\right)^{-1}$.

Let $\widehat{Z}(\mathbf{k})$ and $\widehat{N}(\mathbf{k})$ be the bounded operators in $\mathfrak{H}=L_{2}\left(\Omega ; \mathbb{C}^{n}\right)$ defined by (4.3) and (4.10). Consider the bounded operators $\widehat{\mathcal{Z}}$ and $\widehat{\mathcal{N}}$ in $\mathfrak{G}$ as follows: in the direct integral $\mathcal{K}$ these operators turn into the operators $[\widehat{Z}(\cdot)]$ and $[\widehat{N}(\cdot)]$ of multiplication by $\widehat{Z}(\mathbf{k})$ and $\widehat{N}(\mathbf{k})$, respectively:

$$
\begin{aligned}
\widehat{\mathcal{Z}} & =\mathcal{V}^{-1}[\widehat{Z}(\cdot)] \mathcal{V}, \\
\widehat{\mathcal{N}} & =\mathcal{V}^{-1}[\widehat{N}(\cdot)] \mathcal{V} .
\end{aligned}
$$

Theorem 4.9 and relations (6.1)-(6.3) imply the following representation:

$$
\left(\widehat{\mathcal{A}}+\varepsilon^{2} I\right)^{-1}=\widehat{\mathcal{R}}^{0}(\varepsilon)+\widehat{\mathcal{Z}} \widehat{\mathcal{R}}^{0}(\varepsilon)+\widehat{\mathcal{R}}^{0}(\varepsilon) \widehat{\mathcal{Z}}^{*}-\widehat{\mathcal{R}}^{0}(\varepsilon) \widehat{\mathcal{N}} \widehat{\mathcal{R}}^{0}(\varepsilon)+\widehat{\mathcal{J}}, \quad \varepsilon>0,
$$

where $\widehat{\mathcal{R}}^{0}(\varepsilon)=\left(\widehat{\mathcal{A}}^{0}+\varepsilon^{2} I\right)^{-1}$ and

$$
\widehat{\mathcal{J}}=\mathcal{V}^{-1}\left(\int_{\widetilde{\Omega}} \oplus \widehat{J}(\mathbf{k}) d \mathbf{k}\right) \mathcal{V}
$$

By (4.29), the operator (6.5) admits the estimate

$$
\|\widehat{\mathcal{J}}\|_{\mathfrak{G} \rightarrow \mathfrak{G}} \leq \widehat{\mathcal{C}_{1}}, \quad \varepsilon>0 .
$$

We want to give a direct description of the operators $(6.2),(6.3)$. For this, we need an expression for the operator

$$
\Pi:=\mathcal{V}^{-1}[\widehat{P}] \mathcal{V}
$$

acting in $\mathfrak{G}$. Here $[\widehat{P}]$ is the projection in $\mathcal{K}$ that acts on fibers as the operator $\widehat{P}$ (the operator of averaging over $\Omega$ ). First of all, we calculate the element $[\widehat{P}] \mathcal{V} \mathbf{u} \in \mathcal{K}$ for $\mathbf{u} \in \mathfrak{G}$. Let $\mathcal{F}$ be the Fourier operator in $\mathfrak{G}$. By $(2.9)$, for $\mathbf{k} \in \widetilde{\Omega}$ we have

$$
\begin{aligned}
([\widehat{P}] \mathcal{V} \mathbf{u})(\mathbf{k}) & =|\Omega|^{-1} \int_{\Omega}(\mathcal{V} \mathbf{u})(\mathbf{k}, \mathbf{x}) d \mathbf{x} \\
& =(2 \pi)^{-d / 2}|\Omega|^{-1 / 2} \sum_{\mathbf{a} \in \Gamma} \int_{\Omega} e^{-i\langle\mathbf{k}, \mathbf{x}+\mathbf{a}\rangle} \mathbf{u}(\mathbf{x}+\mathbf{a}) d \mathbf{x}
\end{aligned}
$$

which is equivalent to $([\widehat{P}] \mathcal{V} \mathbf{u})(\mathbf{k})=|\Omega|^{-1 / 2}(\mathcal{F} \mathbf{u})(\mathbf{k}), \mathbf{k} \in \widetilde{\Omega}$.

Now we calculate the quadratic form of the operator $\Pi$, using the fact that $\Pi$ is an orthogonal projection. Let $\chi_{\widetilde{\Omega}}$ be the indicator of the set $\widetilde{\Omega}$, and let $\left[\chi_{\widetilde{\Omega}}\right]$ be the operator of multiplication by $\chi_{\widetilde{\Omega}}$. We have

$$
\begin{aligned}
(\Pi \mathbf{u}, \mathbf{u})_{\mathfrak{G}} & =\|\Pi \mathbf{u}\|_{\mathfrak{G}}^{2}=\|[\widehat{P}] \mathcal{V} \mathbf{u}\|_{\mathcal{K}}^{2}=|\Omega|^{-1} \int_{\Omega} d \mathbf{x} \int_{\widetilde{\Omega}}|(\mathcal{F} \mathbf{u})(\mathbf{k})|^{2} d \mathbf{k} \\
& =\int_{\mathbb{R}^{d}} \chi_{\widetilde{\Omega}}(\mathbf{k})|(\mathcal{F} \mathbf{u})(\mathbf{k})|^{2} d \mathbf{k}=\left(\mathcal{F}^{*}\left[\chi_{\widetilde{\Omega}}\right] \mathcal{F} \mathbf{u}, \mathbf{u}\right)_{\mathfrak{G}}
\end{aligned}
$$


Thus,

$$
\Pi=\mathcal{F}^{*}\left[\chi_{\widetilde{\Omega}}\right] \mathcal{F},
$$

i.e., $\Pi$ is the pseudodifferential operator in $\mathbb{R}^{d}$ with the symbol $\chi_{\widetilde{\Omega}}$.

The operator $\widehat{\mathcal{Z}}$ can easily be calculated by using (6.2) and (6.7). Since $\widehat{\mathcal{Z}}(\mathbf{k})=\Lambda b(\mathbf{k}) \widehat{P}$ by (4.3), and $b(\mathbf{D}+\mathbf{k}) \widehat{P}=b(\mathbf{k}) \widehat{P}$, we see that

$$
\widehat{\mathcal{Z}}=\Lambda b(\mathbf{D}) \Pi \text {. }
$$

Similarly, by (4.10), (6.3), and (6.7),

$$
\widehat{\mathcal{N}}=b(\mathbf{D})^{*} L(\mathbf{D}) b(\mathbf{D}) \Pi .
$$

Note that $b(\mathbf{D})^{*} L(\mathbf{D}) b(\mathbf{D})$ is an $(n \times n)$-matrix third-order DO with constant coefficients. The operators (6.9) and (6.10) are bounded in $\mathfrak{G}$, due to the smoothing operator $\Pi$.

6.2. Approximation of the resolvent $\left(\widehat{\mathcal{A}}+\varepsilon^{2} I\right)^{-1}$. Relations $(6.4),(6.6),(6.9)$, and (6.10) imply the following result.

Theorem 6.1. Let $\widehat{\mathcal{A}}=b(\mathbf{D})^{*} g(\mathbf{x}) b(\mathbf{D})$. Let $g^{0}$ be the effective matrix, and let $\widehat{\mathcal{A}}^{0}=$ $b(\mathbf{D})^{*} g^{0} b(\mathbf{D})$ be the effective operator. Suppose that $\Lambda(\mathbf{x})$ is a periodic $(n \times m)$-matrixvalued function with the columns $\mathbf{v}_{j}(\mathbf{x}), j=1, \ldots, m$, where $\mathbf{v}_{j}$ is the solution of equation (3.4) with $\mathbf{C}=\mathbf{e}_{j}$. Let $L(\mathbf{k})=t L(\boldsymbol{\theta})$ be defined in accordance with (3.5) and (4.8), and let $L(\mathbf{D})$ be the first-order $D O$ with the symbol $L(\mathbf{k})$. Finally, let $\Pi$ be the pseudodifferential operator (6.8). Then for $\varepsilon>0$ we have

$$
\begin{aligned}
\left(\widehat{\mathcal{A}}+\varepsilon^{2} I\right)^{-1}= & \widehat{\mathcal{R}}^{0}(\varepsilon)+\Lambda b(\mathbf{D}) \Pi \widehat{\mathcal{R}}^{0}(\varepsilon)+\widehat{\mathcal{R}}^{0}(\varepsilon)(\Lambda b(\mathbf{D}) \Pi)^{*} \\
& -\widehat{\mathcal{R}}^{0}(\varepsilon) b(\mathbf{D})^{*} L(\mathbf{D}) b(\mathbf{D}) \Pi \widehat{\mathcal{R}}^{0}(\varepsilon)+\widehat{\mathcal{J}},
\end{aligned}
$$

where $\widehat{\mathcal{R}}^{0}(\varepsilon)=\left(\widehat{\mathcal{A}}^{0}+\varepsilon^{2} I\right)^{-1}$, and the operator $\widehat{\mathcal{J}}$ satisfies estimate $(6.6)$ with the constant $\widehat{\mathcal{C}}_{1}$ defined by $(4.29)$.

In a similar way, Theorem 4.10 implies the following result.

Theorem 6.2. Under the assumptions of Theorem 6.1 , suppose that $\Lambda(\mathbf{x}) b(\boldsymbol{\theta})=0$ for all $\boldsymbol{\theta} \in \mathbb{S}^{d-1}, \mathbf{x} \in \Omega$. Then

$$
\left\|\left(\widehat{\mathcal{A}}+\varepsilon^{2} I\right)^{-1}-\left(\widehat{\mathcal{A}}^{0}+\varepsilon^{2} I\right)^{-1}\right\|_{\mathfrak{G} \rightarrow \mathfrak{G}} \leq \widehat{\mathcal{C}}_{2}, \quad \varepsilon>0,
$$

where $\widehat{\mathcal{C}}_{2}$ is the constant defined by (4.30). In particular, $(6.12)$ is valid under conditions (3.9) (i.e., in the case where $g^{0}=\bar{g}$ ).

Observe that conditions (3.9) are equivalent to the relation $\Lambda(\mathbf{x})=0$.

Now we distinguish the case where in (6.11) the term containing the operator $\widehat{\mathcal{N}}=$ $b(\mathbf{D})^{*} L(\mathbf{D}) b(\mathbf{D}) \Pi$ vanishes. The next statement is a consequence of (6.3), Proposition 4.5(2), and Corollary 4.4.

Proposition 6.3. Under the assumptions of Theorem 6.1, suppose that either conditions (3.10) are satisfied (i.e., $g^{0}=g$ ), or $\widehat{\mathcal{A}}=\mathbf{D}^{*} g(\mathbf{x}) \mathbf{D}$, where the matrix $g(\mathbf{x})$ has real entries. Then for $\varepsilon>0$ we have

$$
\left(\widehat{\mathcal{A}}+\varepsilon^{2} I\right)^{-1}=\widehat{\mathcal{R}}^{0}(\varepsilon)+\Lambda b(\mathbf{D}) \Pi \widehat{\mathcal{R}}^{0}(\varepsilon)+\widehat{\mathcal{R}}^{0}(\varepsilon)(\Lambda b(\mathbf{D}) \Pi)^{*}+\widehat{\mathcal{J}},
$$

where $\widehat{\mathcal{J}}$ satisfies (6.6). In particular, this is true if $m=n$. 
6.3. "Elimination" of the operator $\Pi$. Our nearest goal is to find conditions under which $\Pi$ can be replaced by $I$ in (6.11). It turns out that, in the fourth term on the right, such a replacement is always possible (only the constant in the remainder estimate will change), while in the second and the third term $\Pi$ can be replaced by $I$ only under additional conditions on $\Lambda(\mathbf{x})$. We distinguish some cases where these conditions are satisfied a priori.

We consider the operators

$$
\begin{aligned}
& \Sigma_{1}(\varepsilon):=b(\mathbf{D})(I-\Pi) \widehat{\mathcal{R}}^{0}(\varepsilon), \\
& \Sigma_{2}(\varepsilon):=\widehat{\mathcal{R}}^{0}(\varepsilon) b(\mathbf{D})^{*} L(\mathbf{D}) b(\mathbf{D})(I-\Pi) \widehat{\mathcal{R}}^{0}(\varepsilon) .
\end{aligned}
$$

Each of them is a pseudodifferential operator of order $(-1)$ with symbol independent of $\mathbf{x}$. The symbols are given by

$$
\begin{aligned}
\sigma_{1}(\boldsymbol{\xi})= & \left(1-\chi_{\widetilde{\Omega}}(\boldsymbol{\xi})\right) b(\boldsymbol{\xi})\left(b(\boldsymbol{\xi})^{*} g^{0} b(\boldsymbol{\xi})+\varepsilon^{2} \mathbf{1}_{n}\right)^{-1}, \quad \xi \in \mathbb{R}^{d}, \\
\sigma_{2}(\boldsymbol{\xi})= & \left(1-\chi_{\widetilde{\Omega}}(\boldsymbol{\xi})\right)\left(b(\boldsymbol{\xi})^{*} g^{0} b(\boldsymbol{\xi})+\varepsilon^{2} \mathbf{1}_{n}\right)^{-1} \\
& \times b(\boldsymbol{\xi})^{*} L(\boldsymbol{\xi}) b(\boldsymbol{\xi})\left(b(\boldsymbol{\xi})^{*} g^{0} b(\boldsymbol{\xi})+\varepsilon^{2} \mathbf{1}_{n}\right)^{-1}, \quad \xi \in \mathbb{R}^{d} .
\end{aligned}
$$

We show that $\Sigma_{1}(\varepsilon)$ maps $\mathfrak{G}=L_{2}\left(\mathbb{R}^{d} ; \mathbb{C}^{n}\right)$ continuously into $\mathfrak{G}_{*}^{1}=H^{1}\left(\mathbb{R}^{d} ; \mathbb{C}^{m}\right)$, and that the norm of $\Sigma_{1}(\varepsilon)$ is uniformly bounded. Using (2.2) and (4.18), we observe that

$$
b(\xi)^{*} g^{0} b(\xi) \geq|\xi|^{2} \alpha_{0}\left\|g^{-1}\right\|_{L_{\infty}}^{-1} \mathbf{1}_{n}, \quad \xi \in \mathbb{R}^{d} .
$$

Since $|\xi| \geq r_{0}$ for $\xi \in \mathbb{R}^{d} \backslash \widetilde{\Omega}$, relations (6.15) and (6.17) imply that

$$
\left|\sigma_{1}(\xi)\right| \leq|\xi|^{-1} \alpha_{1}^{1 / 2} \alpha_{0}^{-1}\left\|g^{-1}\right\|_{L_{\infty}}, \quad|\xi| \geq r_{0},
$$

and $\sigma_{1}(\xi)=0$ for $|\xi|<r_{0}$. Therefore,

$$
\begin{aligned}
\left\|\Sigma_{1}(\varepsilon)\right\|_{\mathfrak{G} \rightarrow \mathfrak{G}_{*}^{1}} & =\sup _{\xi \in \mathbb{R}^{d}}\left(1+|\xi|^{2}\right)^{1 / 2}\left|\sigma_{1}(\xi)\right| \\
& \leq \alpha_{1}^{1 / 2} \alpha_{0}^{-1}\left\|g^{-1}\right\|_{L_{\infty}} \sup _{|\xi| \geq r_{0}}\left(1+|\xi|^{2}\right)^{1 / 2}|\xi|^{-1} \\
& =\alpha_{1}^{1 / 2} \alpha_{0}^{-1}\left\|g^{-1}\right\|_{L_{\infty}}\left(1+r_{0}^{-2}\right)^{1 / 2} .
\end{aligned}
$$

Similarly, it can be checked that the norm of the operator $\Sigma_{2}(\varepsilon)$ acting from $\mathfrak{G}$ to $\mathfrak{G}^{1}=$ $H^{1}\left(\mathbb{R}^{d} ; \mathbb{C}^{n}\right)$ is uniformly bounded, but for our goals it suffices to estimate the $(\mathfrak{G} \rightarrow \mathfrak{G})$ norm of this operator. The representation (4.9) shows that $\|\widehat{N}(\boldsymbol{\theta})\|=\left|b(\boldsymbol{\theta})^{*} L(\boldsymbol{\theta}) b(\boldsymbol{\theta})\right|$. Hence, combining (4.27) and (4.16), we see that

$$
\begin{aligned}
\left|b(\xi)^{*} L(\xi) b(\xi)\right| & =|\xi|^{3}\left|b(\boldsymbol{\theta})^{*} L(\boldsymbol{\theta}) b(\boldsymbol{\theta})\right| \\
& \leq|\xi|^{3}(2 \widehat{\delta})^{-1 / 2} \alpha_{1}^{3 / 2}\|g\|_{L_{\infty}}^{3 / 2} \\
& =|\xi|^{3} 2^{1 / 2} r_{0}^{-1} \alpha_{0}^{-1 / 2} \alpha_{1}^{3 / 2}\|g\|_{L_{\infty}}^{3 / 2}\left\|g^{-1}\right\|_{L_{\infty}}^{1 / 2}, \quad \xi \in \mathbb{R}^{d} .
\end{aligned}
$$

Then, by (6.16) and (6.17),

$$
\left|\sigma_{2}(\xi)\right| \leq 2^{1 / 2}|\xi|^{-1} r_{0}^{-1} \alpha_{1}^{3 / 2} \alpha_{0}^{-5 / 2}\|g\|_{L_{\infty}}^{3 / 2}\left\|g^{-1}\right\|_{L_{\infty}}^{5 / 2}, \quad|\xi| \geq r_{0},
$$

and $\sigma_{2}(\xi)=0$ for $|\xi|<r_{0}$. Consequently,

$$
\left\|\Sigma_{2}(\varepsilon)\right\|_{\mathfrak{G} \rightarrow \mathfrak{G}}=\sup _{\xi \in \mathbb{R}^{d}}\left|\sigma_{2}(\xi)\right| \leq 2^{1 / 2} \alpha_{1}^{3 / 2} \alpha_{0}^{-5 / 2}\|g\|_{L_{\infty}}^{3 / 2}\left\|g^{-1}\right\|_{L_{\infty}}^{5 / 2} r_{0}^{-2} .
$$

Estimate (6.19) allows us to replace $\Pi$ by $I$ in the fourth term on the right-hand side of (6.11). Theorem 6.1 and estimate (6.19) lead to the following result. 
Theorem 6.4. Under the conditions of Theorem 6.1, for $\varepsilon>0$ we have

$$
\begin{aligned}
\left(\widehat{\mathcal{A}}+\varepsilon^{2} I\right)^{-1}= & \widehat{\mathcal{R}}^{0}(\varepsilon)+\Lambda b(\mathbf{D}) \Pi \widehat{\mathcal{R}}^{0}(\varepsilon) \\
& +\widehat{\mathcal{R}}^{0}(\varepsilon)(\Lambda b(\mathbf{D}) \Pi)^{*}-\widehat{\mathcal{R}}^{0}(\varepsilon) b(\mathbf{D})^{*} L(\mathbf{D}) b(\mathbf{D}) \widehat{\mathcal{R}}^{0}(\varepsilon)+\widehat{\mathcal{J}}^{\prime},
\end{aligned}
$$

and $\widehat{\mathcal{J}}^{\prime}$ satisfies the estimate

$$
\left\|\widehat{\mathcal{J}}^{\prime}\right\|_{\mathfrak{G} \rightarrow \mathfrak{G}} \leq \widehat{\mathcal{C}}_{1}+2^{1 / 2} \alpha_{1}^{3 / 2} \alpha_{0}^{-5 / 2}\|g\|_{L_{\infty}}^{3 / 2}\left\|g^{-1}\right\|_{L_{\infty}}^{5 / 2} r_{0}^{-2}=: \widehat{\mathcal{C}}_{1}^{\prime},
$$

where $\widehat{\mathcal{C}}_{1}$ is the constant defined by (4.29).

Remark 6.5. By Remark 4.11 and (6.21), $\widehat{\mathcal{C}}_{1}^{\prime}$ depends only on $\|g\|_{L_{\infty}},\left\|g^{-1}\right\|_{L_{\infty}}, \alpha_{0}, \alpha_{1}$, and the parameters of the lattice; moreover, this constant, $\widehat{\mathcal{C}}_{1}^{\prime}$, as well as the constant $\widehat{\mathcal{C}_{2}}$ in (6.12), is controlled explicitly.

Under the condition below, it is possible a fortiori to replace $\Pi$ by $I$ in the second and third terms on the right-hand side of (6.11).

Condition 6.6. The operator $[\Lambda]$ of multiplication by the matrix-valued function $\Lambda(\mathbf{x})$ is continuous from $\mathfrak{G}_{*}^{1}$ into $\mathfrak{G}$.

Indeed, under Condition 6.6, by (6.18) we have

$$
\begin{aligned}
\left\|[\Lambda] \Sigma_{1}(\varepsilon)\right\|_{\mathfrak{G} \rightarrow \mathfrak{G}} & \leq\|[\Lambda]\|_{\mathfrak{G}_{*}^{1} \rightarrow \mathfrak{G}}\left\|\Sigma_{1}(\varepsilon)\right\|_{\mathfrak{G} \rightarrow \mathfrak{G}_{*}^{1}} \\
& \leq \alpha_{1}^{1 / 2} \alpha_{0}^{-1}\left\|g^{-1}\right\|_{L_{\infty}}\left(1+r_{0}^{-2}\right)^{1 / 2}\|[\Lambda]\|_{\mathfrak{G}_{*}^{1} \rightarrow \mathfrak{G}} .
\end{aligned}
$$

We give sufficient conditions (in terms of the $L_{p}$-scale) for the validity of Condition 6.6. They are based on the following simple lemma.

Lemma 6.7. Let $\Xi(\mathbf{x})$ be a $\Gamma$-periodic $(n \times m)$-matrix-valued function in $\mathbb{R}^{d}$ such that

$$
\Xi \in L_{p}(\Omega), \quad \begin{cases}p=2 & \text { for } d=1, \\ p>2 & \text { for } d=2, \\ p=d & \text { for } d \geq 3 .\end{cases}
$$

Then the operator $[\Xi]$ of multiplication by $\Xi(\mathbf{x})$ maps $\mathfrak{G}_{*}^{1}$ into $\mathfrak{G}$ continuously, and

$$
\|[\Xi]\|_{\mathfrak{G}_{*}^{1} \rightarrow \mathfrak{G}} \leq c\|\Xi\|_{L_{p}(\Omega)}
$$

where $c=c(m, d, \Omega)$ for $d \neq 2$ and $c=c(m, p, \Omega)$ for $d=2$.

Proof. Let $\mathbf{a} \in \Gamma$, and let $\mathbf{v} \in \mathfrak{G}_{*}^{1}$. Then for $p \geq 2$ we have

$$
\int_{\Omega+\mathbf{a}}|\Xi(\mathbf{x}) \mathbf{v}(\mathbf{x})|^{2} d \mathbf{x} \leq\|\Xi\|_{L_{p}(\Omega)}^{2}\|\mathbf{v}\|_{L_{q}(\Omega+\mathbf{a})}^{2},
$$

where $q=2 p(p-2)^{-1}$. By $(6.23), q=\infty$ for $d=1, q<\infty$ for $d=2$, and $q=2 d(d-2)^{-1}$ for $d \geq 3$. Then the embedding $H^{1}\left(\Omega ; \mathbb{C}^{m}\right) \subset L_{q}\left(\Omega ; \mathbb{C}^{m}\right)$ yields

$$
\|\mathbf{v}\|_{L_{q}\left(\Omega+\mathbf{a} ; \mathbb{C}^{m}\right)} \leq c\|\mathbf{v}\|_{H^{1}\left(\Omega+\mathbf{a} ; \mathbb{C}^{m}\right)} .
$$

From (6.25) and (6.26), summing over $\mathbf{a} \in \Gamma$, we obtain

$$
\int_{\mathbb{R}^{d}}|\Xi(\mathbf{x}) \mathbf{v}(\mathbf{x})|^{2} d \mathbf{x} \leq c^{2}\|\Xi\|_{L_{p}(\Omega)}^{2}\|\mathbf{v}\|_{\mathfrak{G}_{*}^{1}}^{2} .
$$

Now we can prove the following statement.

Proposition 6.8. Let $\Lambda(\mathbf{x})$ be the $\Gamma$-periodic $(n \times m)$-matrix-valued function introduced in Subsection 3.1. Let $d \leq 4$. Then the operator $[\Lambda]$ of multiplication by $\Lambda(\mathbf{x})$ maps $\mathfrak{G}_{*}^{1}$ into $\mathfrak{G}$ continuously, and the norm $\|[\Lambda]\|_{\mathfrak{G}_{*}^{1} \rightarrow \mathfrak{G}}$ is estimated by the constant depending only on $\|g\|_{L_{\infty}},\left\|g^{-1}\right\|_{L_{\infty}}, \alpha_{0}, m, n, d$, and on the lattice $\Gamma$. 
Proof. The columns $\mathbf{v}_{j}(\mathbf{x}), j=1, \ldots, m$, of the matrix $\Lambda(\mathbf{x})$ are the solutions of equation (3.4) with $\mathbf{C}=\mathbf{e}_{j}$. Thus, they belong to $\widetilde{H}^{1}\left(\Omega ; \mathbb{C}^{n}\right)$. Therefore, $\Lambda \in L_{\infty}$ for $d=1$, $\Lambda \in L_{s}(\Omega)$ with any $s<\infty$ for $d=2$, and $\Lambda \in L_{2 d /(d-2)}(\Omega)$ for $d \geq 3$. So, a fortiori, $\Lambda(\mathbf{x})$ satisfies the conditions of Lemma 6.7 for $d \leq 4$, and

$$
\|\Lambda\|_{L_{p}(\Omega)} \leq \tilde{c}\|\Lambda\|_{H^{1}(\Omega)},
$$

where $\tilde{c}=\tilde{c}(d, m, n, \Omega)$ for $d \neq 2$ and $\tilde{c}=\tilde{c}(p, m, n, \Omega)$ for $d=2$. Then, by (6.24),

$$
\|[\Lambda]\|_{\mathfrak{G}_{*}^{1} \rightarrow \mathfrak{G}} \leq c \tilde{c}\|\Lambda\|_{H^{1}(\Omega)} .
$$

Fixing $p>2$ arbitrarily for $d=2$, we may assume that the constant in (6.27) depends only on $d, m, n$, and $\Omega$.

Next, obviously, the solutions $\mathbf{v}_{j}$ of equation (3.4) with $\mathbf{C}=\mathbf{e}_{j}$ satisfy the "energy" estimate

$$
\left\|\mathbf{D v}_{j}\right\|_{L_{2}(\Omega)} \leq \alpha_{0}^{-1 / 2}|\Omega|^{1 / 2}\|g\|_{L_{\infty}}^{1 / 2}\left\|g^{-1}\right\|_{L_{\infty}}^{1 / 2} .
$$

Moreover, for any function $\mathbf{v} \in \widetilde{H}^{1}\left(\Omega ; \mathbb{C}^{n}\right)$ with zero mean, we have

$$
\|\mathbf{v}\|_{L_{2}(\Omega)} \leq\left(2 r_{0}\right)^{-1}\|\mathbf{D v}\|_{L_{2}(\Omega)} .
$$

Hence,

Therefore,

$$
\left\|\mathbf{v}_{j}\right\|_{H^{1}(\Omega)} \leq\left(1+1 / 4 r_{0}^{2}\right)^{1 / 2} \alpha_{0}^{-1 / 2}|\Omega|^{1 / 2}\|g\|_{L_{\infty}}^{1 / 2}\left\|g^{-1}\right\|_{L_{\infty}}^{1 / 2} .
$$

$$
\begin{aligned}
\|\Lambda\|_{H^{1}(\Omega)} & \leq\left(\sum_{j=1}^{m}\left\|\mathbf{v}_{j}\right\|_{H^{1}(\Omega)}^{2}\right)^{1 / 2} \\
& \leq m^{1 / 2}\left(1+1 / 4 r_{0}^{2}\right)^{1 / 2} \alpha_{0}^{-1 / 2}|\Omega|^{1 / 2}\|g\|_{L_{\infty}}^{1 / 2}\left\|g^{-1}\right\|_{L_{\infty}}^{1 / 2} .
\end{aligned}
$$

Combining this with (6.27), we complete the proof.

Besides the case where $d \leq 4$, the conditions of Lemma 6.7 are satisfied in any dimension for the scalar elliptic operator $\widehat{\mathcal{A}}(g)=\mathbf{D}^{*} g(\mathbf{x}) \mathbf{D}$, where the matrix $g(\mathbf{x})$ has real entries. In this case we have $\mathbf{v}_{j} \in L_{\infty}$ (and then also $\Lambda \in L_{\infty}$ ), and the norm $\left\|\mathbf{v}_{j}\right\|_{L_{\infty}}$ is estimated by a constant depending only on $\|g\|_{L_{\infty}},\left\|g^{-1}\right\|_{L_{\infty}}, d$, and $\Omega$. This fact follows from LaU, Chapter III, Theorem 13.1].

Yet another case where Condition 6.6 is satisfied a fortiori, is indicated in the following statement.

Proposition 6.9. Let $\mathbf{v}_{j} \in \widetilde{H}^{1}\left(\Omega ; \mathbb{C}^{n}\right)$ be the solution of problem (3.4) with $\mathbf{C}=\mathbf{e}_{j}$. Suppose that conditions (3.10) are satisfied (i.e., $g^{0}=\underline{g}$ ). Then $\mathbf{v}_{j} \in L_{\infty}$, and the norm $\left\|\mathbf{v}_{j}\right\|_{L_{\infty}}$ is estimated by a constant depending only on $\|g\|_{L_{\infty}},\left\|g^{-1}\right\|_{L_{\infty}}, \alpha_{0}, \alpha_{1}, m, n, d$, and the parameters of the lattice $\Gamma$.

Proof. Under conditions (3.10), the vector-valued function $g(\mathbf{x})\left(b(\mathbf{D}) \mathbf{v}_{j}+\mathbf{e}_{j}\right)=\mathbf{C}_{j}$ is constant (see Remark 3.5), and $\mathbf{C}_{j}=g \mathbf{e}_{j}$. Then $b(\mathbf{D}) \mathbf{v}_{j}=g(\mathbf{x})^{-1} \mathbf{C}_{j}-\mathbf{e}_{j}$, so that $\mathbf{v}_{j} \in \widetilde{H}^{1}\left(\Omega ; \mathbb{C}^{n}\right)$ is the periodic solution of the problem

$$
b(\mathbf{D})^{*} b(\mathbf{D}) \mathbf{v}_{j}=b(\mathbf{D})^{*}\left(g(\mathbf{x})^{-1} \mathbf{C}_{j}-\mathbf{e}_{j}\right), \quad \int_{\Omega} \mathbf{v}_{j}(\mathbf{x}) d \mathbf{x}=0 .
$$

The operator $b(\mathbf{D})^{*} b(\mathbf{D})$ is a matrix elliptic operator with constant coefficients. Therefore, equation (6.31) can be solved in terms of Fourier series expansions. Since $\mathbf{w}_{j}:=$ $g^{-1} \mathbf{C}_{j}-\mathbf{e}_{j} \in L_{\infty}$, for any $p \in(1, \infty)$ the well-known Marcinkiewicz multiplier theorem for Fourier series is applicable (see [Ma], or the Appendix in the book Mi]). This theorem implies that $\mathbf{v}_{j} \in W_{p}^{1}(\Omega)$ for $1<p<\infty$ and that $\left\|\mathbf{v}_{j}\right\|_{W_{p}^{1}(\Omega)} \leq C\left\|\mathbf{w}_{j}\right\|_{L_{p}(\Omega)}$, where $C$ depends on $\alpha_{0}, \alpha_{1}, m, n, d, p$, and the parameters of $\Gamma$. If $p>d$, it follows 
that $\mathbf{v}_{j} \in L_{\infty}$, and the norm $\left\|\mathbf{v}_{j}\right\|_{L_{\infty}}$ is estimated by a constant depending on $\|g\|_{L_{\infty}}$, $\left\|g^{-1}\right\|_{L_{\infty}}, \alpha_{0}, \alpha_{1}, m, n, d$, and the parameters of $\Gamma$.

Thus, Condition 6.6 is guaranteed by the following (more restrictive) condition.

Condition 6.10. At least one of the following three assertions is true: 1) the dimension $d$ is at most $4 ; 2$ ) the representations (3.10) are valid (or equivalently, $g^{0}=g$ ); 3 ) $\widehat{\mathcal{A}}(g)=$ $\mathbf{D}^{*} g \mathbf{D}$, where the matrix $g(\mathbf{x})$ has real entries.

Remark 6.11. Condition 6.6 can also be ensured by some smoothness assumptions on the matrix $g(\mathbf{x})$.

Now, combining Theorem 6.4 with (6.13) and (6.22), we immediately obtain the following result.

Theorem 6.12. Under the conditions of Theorem 6.1, suppose that Condition 6.6 or (the more restrictive) Condition 6.10 is satisfied. Then for $\varepsilon>0$ we have

$$
\begin{aligned}
\left(\widehat{\mathcal{A}}+\varepsilon^{2} I\right)^{-1}= & \widehat{\mathcal{R}}^{0}(\varepsilon)+\Lambda b(\mathbf{D}) \widehat{\mathcal{R}}^{0}(\varepsilon)+\widehat{\mathcal{R}}^{0}(\varepsilon) b(\mathbf{D})^{*} \Lambda^{*} \\
& -\widehat{\mathcal{R}}^{0}(\varepsilon) b(\mathbf{D})^{*} L(\mathbf{D}) b(\mathbf{D}) \widehat{\mathcal{R}}^{0}(\varepsilon)+\widehat{\mathcal{J}}^{\prime \prime}
\end{aligned}
$$

where $\widehat{\mathcal{J}}^{\prime \prime}$ satisfies the estimate

$$
\left\|\widehat{\mathcal{J}}^{\prime \prime}\right\|_{\mathfrak{G} \rightarrow \mathfrak{G}} \leq \widehat{\mathcal{C}}_{1}^{\prime}+2 \alpha_{1}^{1 / 2} \alpha_{0}^{-1}\left\|g^{-1}\right\|_{L_{\infty}}\left(1+r_{0}^{-2}\right)^{1 / 2}\|[\Lambda]\|_{\mathfrak{G}_{*}^{1} \rightarrow \mathfrak{G}}=: \widehat{\mathcal{C}}_{1}^{\prime \prime} .
$$

Here the constant $\widehat{\mathcal{C}}_{1}^{\prime}$ is defined by (6.21). Under Condition 6.10, the constant $\widehat{\mathcal{C}}_{1}^{\prime \prime}$ depends on $\|g\|_{L_{\infty}},\left\|g^{-1}\right\|_{L_{\infty}}, \alpha_{0}, \alpha_{1}, m, n, d$, and the parameters of the lattice $\Gamma$.

Finally, combining Proposition 6.3 and Condition 6.10, we arrive at the following statement.

Proposition 6.13. Under the assumptions of Theorem 6.1, suppose that one of the following two conditions is fulfilled:

$1^{\circ}$. relations $(3.10)$ are satisfied (i.e., $g^{0}=\underline{g}$ );

$2^{\circ} . \widehat{\mathcal{A}}=\mathbf{D}^{*} g(\mathbf{x}) \mathbf{D}$, where the matrix $g(\mathbf{x})$ has real entries.

Then for $\varepsilon>0$ we have

$$
\left(\widehat{\mathcal{A}}+\varepsilon^{2} I\right)^{-1}=\widehat{\mathcal{R}}^{0}(\varepsilon)+\Lambda b(\mathbf{D}) \widehat{\mathcal{R}}^{0}(\varepsilon)+\widehat{\mathcal{R}}^{0}(\varepsilon) b(\mathbf{D})^{*} \Lambda^{*}+\widehat{\mathcal{J}}^{\times}
$$

and

$$
\left\|\widehat{\mathcal{J}}^{\times}\right\|_{\mathfrak{G} \rightarrow \mathfrak{G}} \leq \widehat{\mathcal{C}}_{1}+2 \alpha_{1}^{1 / 2} \alpha_{0}^{-1}\left\|g^{-1}\right\|_{L_{\infty}}\left(1+r_{0}^{-2}\right)^{1 / 2}\|[\Lambda]\|_{\mathfrak{G}_{*}^{1} \rightarrow \mathfrak{G}}=: \widehat{\mathcal{C}}_{1}^{\times},
$$

where $\widehat{\mathcal{C}}_{1}$ is the constant defined by (4.29). The constant $\widehat{\mathcal{C}}_{1}^{\times}$depends only on $\|g\|_{L_{\infty}}$, $\left\|g^{-1}\right\|_{L_{\infty}}, \alpha_{0}, \alpha_{1}, m, n, d$, and the parameters of the lattice $\Gamma$.

\section{§7. Approximation of the generalized Resolvent $\left(\widehat{\mathcal{A}}+\varepsilon^{2} Q\right)^{-1}$}

7.1. In the study of the generalized resolvent, for references and further applications it is convenient to fix some factorization of the periodic matrix $Q(\mathbf{x})$ in the form (5.1):

$$
Q(\mathbf{x})=\left(f(\mathbf{x}) f(\mathbf{x})^{*}\right)^{-1},
$$

where the periodic matrix-valued function $f$ satisfies condition (2.3), i.e., $f, f^{-1} \in L_{\infty}$. The results do not depend on the choice of this factorization.

By analogy with the deduction of Theorem 6.1 from Theorem 4.9, the Gelfand transformation allows us to deduce the following result from Theorem 5.8. 
Theorem 7.1. Let $\widehat{\mathcal{A}}=\widehat{\mathcal{A}}(g)=b(\mathbf{D})^{*} g(\mathbf{x}) b(\mathbf{D})$. Let $g^{0}$ be the effective matrix, and let $\widehat{\mathcal{A}}^{0}=\widehat{\mathcal{A}}\left(g^{0}\right)$ be the effective operator. Suppose that $Q(\mathbf{x})$ is a periodic positive $(n \times n)$ matrix-valued function such that $Q, Q^{-1} \in L_{\infty}$. We put $\bar{Q}=|\Omega|^{-1} \int_{\Omega} Q(\mathbf{x}) d \mathbf{x}$. Suppose that $\Lambda_{Q}(\mathbf{x})$ is a periodic $(n \times m)$-matrix-valued function with columns $\mathbf{v}_{j}^{(Q)}(\mathbf{x}), j=$ $1, \ldots, m$, where $\mathbf{v}_{j}^{(Q)}$ is the solution of equation (5.3) with $\mathbf{C}=\mathbf{e}_{j}$. Let the matrix-valued function $L_{Q}(\mathbf{k})=t L_{Q}(\boldsymbol{\theta})$ be defined by (3.5) and (5.11), let $L_{Q}(\mathbf{D})$ be the first order DO with the symbol $L_{Q}(\mathbf{k})$, and let $\Pi$ be the pseudodifferential operator (6.8). Then for $\varepsilon>0$ we have

$$
\left(\widehat{\mathcal{A}}+\varepsilon^{2} Q\right)^{-1}=\widehat{\mathcal{R}}_{Q}^{0}(\varepsilon)+\mathfrak{K}_{Q}(\varepsilon)+\check{\mathcal{J}},
$$

where $\widehat{\mathcal{R}}_{Q}^{0}(\varepsilon)=\left(\widehat{\mathcal{A}}^{0}+\varepsilon^{2} \bar{Q}\right)^{-1}$, and the "corrector" $\mathfrak{K}_{Q}(\varepsilon)$ is given by the expression

$$
\begin{aligned}
\mathfrak{K}_{Q}(\varepsilon)= & \Lambda_{Q} b(\mathbf{D}) \Pi \widehat{\mathcal{R}}_{Q}^{0}(\varepsilon)+\widehat{\mathcal{R}}_{Q}^{0}(\varepsilon)\left(\Lambda_{Q} b(\mathbf{D}) \Pi\right)^{*} \\
& -\widehat{\mathcal{R}}_{Q}^{0}(\varepsilon) b(\mathbf{D})^{*} L_{Q}(\mathbf{D}) b(\mathbf{D}) \Pi \widehat{\mathcal{R}}_{Q}^{0}(\varepsilon) .
\end{aligned}
$$

The remainder term $\check{\mathcal{J}}$ satisfies the estimate

$$
\|\check{\mathcal{J}}\|_{\mathfrak{G} \rightarrow \mathfrak{G}} \leq \mathcal{C}_{1},
$$

where $\mathcal{C}_{1}$ is the constant defined by (5.24).

Similarly, Theorem 5.9 implies the following statement.

Theorem 7.2. Under the assumptions of Theorem 7.1, suppose that $\Lambda(\mathbf{x}) b(\boldsymbol{\theta})=0$ for all $\boldsymbol{\theta} \in \mathbb{S}^{d-1}, \mathrm{x} \in \Omega$. Then

$$
\left\|\left(\widehat{\mathcal{A}}+\varepsilon^{2} Q\right)^{-1}-\left(\widehat{\mathcal{A}}^{0}+\varepsilon^{2} \bar{Q}\right)^{-1}\right\|_{\mathfrak{G} \rightarrow \mathfrak{G}} \leq \mathcal{C}_{2}, \quad \varepsilon>0,
$$

where $\mathcal{C}_{2}$ is the constant defined by (5.25). In particular, this is true under conditions (3.9) (i.e., if $g^{0}=\bar{g}$ ).

Consider the situation where the third term in (7.2) is zero. This is the case for the $Q$-resolvent of the operator $\widehat{\mathcal{A}}=\mathbf{D}^{*} g(\mathbf{x}) \mathbf{D}$ if the matrix $g(\mathbf{x})$ and the function $Q(\mathbf{x})$ are real. Here we can either refer to Corollary 5.4 or use the representation (5.11) for the symbol $L_{Q}(\boldsymbol{\theta})$. Indeed, now the row-matrix $\Lambda_{Q}(\mathbf{x})$ is purely imaginary, while the matrix $\widetilde{g}(\mathbf{x})$ has real entries, and $b(\boldsymbol{\theta})=\boldsymbol{\theta}$. Therefore, $L_{Q}(\boldsymbol{\theta})$ is a Hermitian matrix with purely imaginary entries, whence the number $b(\boldsymbol{\theta})^{*} L_{Q}(\boldsymbol{\theta}) b(\boldsymbol{\theta})=\boldsymbol{\theta}^{*} L_{Q}(\boldsymbol{\theta}) \boldsymbol{\theta}$ is equal to zero. In this example, by (5.4), expression (7.2) takes the form

$$
\begin{aligned}
\mathfrak{K}_{Q}(\varepsilon) & =\left(\Lambda+\Lambda_{Q}^{0}\right) \mathbf{D} \Pi \widehat{\mathcal{R}}_{Q}^{0}(\varepsilon)+\widehat{\mathcal{R}}_{Q}^{0}(\varepsilon)\left(\left(\Lambda+\Lambda_{Q}^{0}\right) \mathbf{D} \Pi\right)^{*} \\
& =\Lambda \mathbf{D} \Pi \widehat{\mathcal{R}}_{Q}^{0}(\varepsilon)+\widehat{\mathcal{R}}_{Q}^{0}(\varepsilon)(\Lambda \mathbf{D} \Pi)^{*} .
\end{aligned}
$$

We have used the fact that $\Lambda_{Q}^{0}$ is a constant row-matrix with purely imaginary entries, whence $\Lambda_{Q}^{0} \mathbf{D} \widehat{\mathcal{R}}_{Q}^{0}(\varepsilon)+\widehat{\mathcal{R}}_{Q}^{0}(\varepsilon)\left(\Lambda_{Q}^{0} \mathbf{D}\right)^{*}=0$. As a result, we obtain the following statement.

Proposition 7.3. Suppose that the conditions of Theorem 7.1 are satisfied, that $\widehat{\mathcal{A}}=$ $\mathbf{D}^{*} g(\mathbf{x}) \mathbf{D}$, the matrix $g(\mathbf{x})$ has real entries, and $Q(\mathbf{x})$ is real-valued. Then for $\varepsilon>0$ we have

$$
\left(\widehat{\mathcal{A}}+\varepsilon^{2} Q\right)^{-1}=\widehat{\mathcal{R}}_{Q}^{0}(\varepsilon)+\Lambda \mathbf{D} \Pi \widehat{\mathcal{R}}_{Q}^{0}(\varepsilon)+\widehat{\mathcal{R}}_{Q}^{0}(\varepsilon)(\Lambda \mathbf{D} \Pi)^{*}+\check{\mathcal{J}}
$$

where $\check{\mathcal{J}}$ satisfies estimate (7.3). 
7.2. Transformation of the expression (7.2). To transform $\mathfrak{K}_{Q}(\varepsilon)$, we use relations (5.4) and (5.12). We have

$$
\mathfrak{K}_{Q}(\varepsilon)=\mathfrak{K}_{1}(\varepsilon)+\mathfrak{K}_{2}(\varepsilon),
$$

where

$$
\begin{aligned}
\mathfrak{K}_{1}(\varepsilon)= & \Lambda b(\mathbf{D}) \Pi \widehat{\mathcal{R}}_{Q}^{0}(\varepsilon)+\widehat{\mathcal{R}}_{Q}^{0}(\varepsilon)(\Lambda b(\mathbf{D}) \Pi)^{*}-\widehat{\mathcal{R}}_{Q}^{0}(\varepsilon) b(\mathbf{D})^{*} L(\mathbf{D}) b(\mathbf{D}) \Pi \widehat{\mathcal{R}}_{Q}^{0}(\varepsilon), \\
\mathfrak{K}_{2}(\varepsilon)= & \Lambda_{Q}^{0} b(\mathbf{D}) \Pi \widehat{\mathcal{R}}_{Q}^{0}(\varepsilon)+\widehat{\mathcal{R}}_{Q}^{0}(\varepsilon) \Pi b(\mathbf{D})^{*}\left(\Lambda_{Q}^{0}\right)^{*} \\
& -\widehat{\mathcal{R}}_{Q}^{0}(\varepsilon) b(\mathbf{D})^{*}\left(\left(\Lambda_{Q}^{0}\right)^{*} b(\mathbf{D})^{*} g^{0}+g^{0} b(\mathbf{D}) \Lambda_{Q}^{0}\right) b(\mathbf{D}) \Pi \widehat{\mathcal{R}}_{Q}^{0}(\varepsilon) .
\end{aligned}
$$

The operator (7.5) can be written as follows:

$$
\begin{aligned}
\mathfrak{K}_{2}(\varepsilon)= & \Lambda_{Q}^{0} b(\mathbf{D}) \Pi \widehat{\mathcal{R}}_{Q}^{0}(\varepsilon)+\widehat{\mathcal{R}}_{Q}^{0}(\varepsilon) \Pi b(\mathbf{D})^{*}\left(\Lambda_{Q}^{0}\right)^{*} \\
& -\widehat{\mathcal{R}}_{Q}^{0}(\varepsilon) b(\mathbf{D})^{*}\left(\Lambda_{Q}^{0}\right)^{*}\left(\widehat{\mathcal{A}}^{0}+\varepsilon^{2} \bar{Q}-\varepsilon^{2} \bar{Q}\right) \Pi \widehat{\mathcal{R}}_{Q}^{0}(\varepsilon) \\
& -\widehat{\mathcal{R}}_{Q}^{0}(\varepsilon)\left(\widehat{\mathcal{A}}^{0}+\varepsilon^{2} \bar{Q}-\varepsilon^{2} \bar{Q}\right) \Lambda_{Q}^{0} b(\mathbf{D}) \Pi \widehat{\mathcal{R}}_{Q}^{0}(\varepsilon) \\
= & \widehat{\mathcal{R}}_{Q}^{0}(\varepsilon)\left(b(\mathbf{D})^{*}\left(\Lambda_{Q}^{0}\right)^{*} \varepsilon^{2} \bar{Q}+\varepsilon^{2} \bar{Q} \Lambda_{Q}^{0} b(\mathbf{D})\right) \Pi \widehat{\mathcal{R}}_{Q}^{0}(\varepsilon) .
\end{aligned}
$$

Finally, since $\bar{Q} \Lambda_{Q}^{0}=-\overline{Q \Lambda}$ (see (5.4)), we obtain

$$
\begin{aligned}
\mathfrak{K}_{Q}(\varepsilon)= & \Lambda b(\mathbf{D}) \Pi \widehat{\mathcal{R}}_{Q}^{0}(\varepsilon)+\widehat{\mathcal{R}}_{Q}^{0}(\varepsilon)(\Lambda b(\mathbf{D}) \Pi)^{*} \\
& -\widehat{\mathcal{R}}_{Q}^{0}(\varepsilon)\left(b(\mathbf{D})^{*} L(\mathbf{D}) b(\mathbf{D})+\varepsilon^{2} b(\mathbf{D})^{*}(\overline{Q \Lambda})^{*}+\varepsilon^{2}(\overline{Q \Lambda}) b(\mathbf{D})\right) \Pi \widehat{\mathcal{R}}_{Q}^{0}(\varepsilon) .
\end{aligned}
$$

In comparison with (7.2), the representation (7.6) is more convenient, because it involves $\Lambda$ and $L(\mathbf{D})$ (instead of $\Lambda_{Q}$ and $L_{Q}(\mathbf{D})$ in (7.2)). For further applications (see Subsection 9.5), it is important that in the first two terms in (7.6) the factor $\Lambda$ has zero mean value. On the other hand, the representation (7.1) with the "corrector" $\mathfrak{K}_{Q}(\varepsilon)$ written in the form (7.2) has its own advantages. First, this representation is parallel to the representation (6.11) for the resolvent $\left(\widehat{\mathcal{A}}+\varepsilon^{2} I\right)^{-1}$. Second, the operator $\widehat{N}_{Q}(\boldsymbol{\theta})=$ $b(\boldsymbol{\theta})^{*} L_{Q}(\boldsymbol{\theta}) b(\boldsymbol{\theta}) \widehat{P}$, which gives rise to the third term on the right-hand side of (7.2), contains information about the coefficients in the expansions of the analytic branches of eigenvalues and eigenvectors of the operator $\mathcal{A}(\mathbf{k})=f^{*} \widehat{\mathcal{A}}(\mathbf{k}) f$. Therefore, in what follows we shall use both representations (7.2) and (7.6) for $\mathfrak{K}_{Q}(\varepsilon)$.

7.3. "Elimination" of the operator П. By analogy with (6.13) and (6.14), we introduce the operators

$$
\begin{aligned}
& \Sigma_{1}^{(Q)}(\varepsilon):=b(\mathbf{D})(I-\Pi) \widehat{\mathcal{R}}_{Q}^{0}(\varepsilon), \\
& \Sigma_{2}^{(Q)}(\varepsilon):=\widehat{\mathcal{R}}_{Q}^{0}(\varepsilon) b(\mathbf{D})^{*} L_{Q}(\mathbf{D}) b(\mathbf{D})(I-\Pi) \widehat{\mathcal{R}}_{Q}^{0}(\varepsilon) .
\end{aligned}
$$

Also, we consider the operator

$$
\begin{aligned}
\Sigma_{3}^{(Q)}(\varepsilon):=\widehat{\mathcal{R}}_{Q}^{0}(\varepsilon)( & b(\mathbf{D})^{*} L(\mathbf{D}) b(\mathbf{D}) \\
& \left.+\varepsilon^{2} b(\mathbf{D})^{*}(\overline{Q \Lambda})^{*}+\varepsilon^{2}(\overline{Q \Lambda}) b(\mathbf{D})\right)(I-\Pi) \widehat{\mathcal{R}}_{Q}^{0}(\varepsilon),
\end{aligned}
$$

which can be rewritten in the form

$$
\begin{aligned}
\Sigma_{3}^{(Q)}(\varepsilon)= & \widehat{\mathcal{R}}_{Q}^{0}(\varepsilon) b(\mathbf{D})^{*} L(\mathbf{D}) b(\mathbf{D})(I-\Pi) \widehat{\mathcal{R}}_{Q}^{0}(\varepsilon) \\
& +\varepsilon^{2}\left(\Sigma_{1}^{(Q)}(\varepsilon)\right)^{*}(\overline{Q \Lambda})^{*} \widehat{\mathcal{R}}_{Q}^{0}(\varepsilon)+\varepsilon^{2} \widehat{\mathcal{R}}_{Q}^{0}(\varepsilon)(\overline{Q \Lambda}) \Sigma_{1}^{(Q)}(\varepsilon) .
\end{aligned}
$$

The operator (7.7) admits the same estimate as $\Sigma_{1}(\varepsilon)$ (cf. (6.17), (6.18)):

$$
\left\|\Sigma_{1}^{(Q)}(\varepsilon)\right\|_{{\mathfrak{G} \rightarrow \mathfrak{G}_{*}}_{*}} \leq \alpha_{1}^{1 / 2} \alpha_{0}^{-1}\left\|g^{-1}\right\|_{L_{\infty}}\left(1+r_{0}^{-2}\right)^{1 / 2} .
$$


The operator (7.8) is estimated in the same way as $\Sigma_{2}(\varepsilon)$. Namely, (5.13) and (5.15) imply that

$$
\left|b(\boldsymbol{\xi})^{*} L_{Q}(\xi) b(\xi)\right|=|\xi|^{3}\left|b(\boldsymbol{\theta})^{*} L_{Q}(\boldsymbol{\theta}) b(\boldsymbol{\theta})\right| \leq C_{2}^{(Q)}|\xi|^{3},
$$

where $C_{2}^{(Q)}$ is the constant defined by (5.15). As in the proof of (6.19), combining this with (6.17) yields

$$
\begin{aligned}
\left\|\Sigma_{2}^{(Q)}(\varepsilon)\right\|_{\mathfrak{G} \rightarrow \mathfrak{G}} & \leq C_{2}^{(Q)} \alpha_{0}^{-2}\left\|g^{-1}\right\|_{L_{\infty}}^{2} r_{0}^{-1} \\
& =2^{1 / 2} \alpha_{1}^{3 / 2} \alpha_{0}^{-5 / 2}\|g\|_{L_{\infty}}^{3 / 2}\left\|g^{-1}\right\|_{L_{\infty}}^{5 / 2}\|f\|_{L_{\infty}}^{2}\left\|f^{-1}\right\|_{L_{\infty}}^{2} r_{0}^{-2} .
\end{aligned}
$$

Next, the first term on the right-hand side of (7.9) admits the same estimate as $\Sigma_{2}(\varepsilon)$ (cf. (6.19)):

$$
\left\|\widehat{\mathcal{R}}_{Q}^{0}(\varepsilon) b(\mathbf{D})^{*} L(\mathbf{D}) b(\mathbf{D})(I-\Pi) \widehat{\mathcal{R}}_{Q}^{0}(\varepsilon)\right\|_{\mathfrak{G} \rightarrow \mathfrak{G}} \leq 2^{1 / 2} \alpha_{1}^{3 / 2} \alpha_{0}^{-5 / 2}\|g\|_{L_{\infty} / 2}^{3 / 2}\left\|g^{-1}\right\|_{L_{\infty}}^{5 / 2} r_{0}^{-2} .
$$

For estimating the two other terms in (7.9), we use the elementary inequality

$$
\left\|\varepsilon^{2} \widehat{\mathcal{R}}_{Q}^{0}(\varepsilon)\right\|_{\mathfrak{G} \rightarrow \mathfrak{G}} \leq\left\|Q^{-1}\right\|_{L_{\infty}}
$$

and also an estimate for the norm of the constant matrix $(\overline{Q \Lambda})$. From (6.28) and (6.29), it follows (cf. (6.30)) that

$$
\|\Lambda\|_{L_{2}(\Omega)} \leq m^{1 / 2}\left(2 r_{0}\right)^{-1} \alpha_{0}^{-1 / 2}|\Omega|^{1 / 2}\|g\|_{L_{\infty}}^{1 / 2}\left\|g^{-1}\right\|_{L_{\infty}}^{1 / 2} .
$$

We have

$$
\begin{aligned}
|\overline{Q \Lambda}| & \leq|\Omega|^{-1}\|Q\|_{L_{\infty}} \int_{\Omega}|\Lambda| d \mathbf{x} \leq|\Omega|^{-1 / 2}\|Q\|_{L_{\infty}}\|\Lambda\|_{L_{2}(\Omega)} \\
& \leq m^{1 / 2}\left(2 r_{0}\right)^{-1} \alpha_{0}^{-1 / 2}\|g\|_{L_{\infty}}^{1 / 2}\left\|g^{-1}\right\|_{L_{\infty}}^{1 / 2}\|Q\|_{L_{\infty}} .
\end{aligned}
$$

Now, (7.9), (7.10), and (7.12)-(7.14) imply that

$$
\begin{aligned}
\left\|\Sigma_{3}^{(Q)}(\varepsilon)\right\|_{\mathfrak{G} \rightarrow \mathfrak{G}} & \\
\leq & 2^{1 / 2} \alpha_{1}^{3 / 2} \alpha_{0}^{-5 / 2}\|g\|_{L_{\infty}}^{3 / 2}\left\|g^{-1}\right\|_{L_{\infty}}^{5 / 2} r_{0}^{-2} \\
& \quad+m^{1 / 2} \alpha_{1}^{1 / 2} \alpha_{0}^{-3 / 2}\|g\|_{L_{\infty}}^{1 / 2}\left\|g^{-1}\right\|_{L_{\infty} / 2}^{3 / 2}\|Q\|_{L_{\infty}}\left\|Q^{-1}\right\|_{L_{\infty}}\left(1+r_{0}^{-2}\right)^{1 / 2} r_{0}^{-1}=: \mathfrak{C} .
\end{aligned}
$$

Estimates (7.11) and (7.15) allow us to replace $\Pi$ by $I$ in the third term in (7.2) and in the third term in (7.6), respectively. The following theorem is a direct consequence of Theorem 7.1, the representation (7.6), and estimates (7.11), (7.15).

Theorem 7.4. Let the operators $\widehat{\mathcal{A}}, \widehat{\mathcal{A}}^{0}$ and the matrix-valued function $Q(\mathbf{x})$ be the same as in Theorem 7.1. Suppose that $\Lambda(\mathbf{x})$ is the periodic $(n \times m)$-matrix-valued function with the columns $\mathbf{v}_{j}(\mathbf{x}), j=1, \ldots, m$, where $\mathbf{v}_{j}$ is the solution of problem (3.4) with $\mathbf{C}=\mathbf{e}_{j}$. Let $\Lambda_{Q}(\mathbf{x})$ be the matrix-valued function defined by (5.4). Let $L(\mathbf{k})=t L(\boldsymbol{\theta})$ and $L_{Q}(\mathbf{k})=t L_{Q}(\boldsymbol{\theta})$ be the matrix-valued functions defined by (3.5), (4.8), and (5.12), let $L(\mathbf{D})$ and $L_{Q}(\mathbf{D})$ be the DOs with the symbols $L(\mathbf{k})$ and $L_{Q}(\mathbf{k})$, respectively, and let $\Pi$ be the pseudodifferential operator (6.8). Then for $\varepsilon>0$ we have

$$
\begin{aligned}
\left(\widehat{\mathcal{A}}+\varepsilon^{2} Q\right)^{-1}= & \widehat{\mathcal{R}}_{Q}^{0}(\varepsilon)+\Lambda_{Q} b(\mathbf{D}) \Pi \widehat{\mathcal{R}}_{Q}^{0}(\varepsilon)+\widehat{\mathcal{R}}_{Q}^{0}(\varepsilon)\left(\Lambda_{Q} b(\mathbf{D}) \Pi\right)^{*} \\
& -\widehat{\mathcal{R}}_{Q}^{0}(\varepsilon) b(\mathbf{D})^{*} L_{Q}(\mathbf{D}) b(\mathbf{D}) \widehat{\mathcal{R}}_{Q}^{0}(\varepsilon)+\check{\mathcal{J}}^{\prime} \\
\left(\widehat{\mathcal{A}}+\varepsilon^{2} Q\right)^{-1}= & \widehat{\mathcal{R}}_{Q}^{0}(\varepsilon)+\Lambda b(\mathbf{D}) \Pi \widehat{\mathcal{R}}_{Q}^{0}(\varepsilon)+\widehat{\mathcal{R}}_{Q}^{0}(\varepsilon)(\Lambda b(\mathbf{D}) \Pi)^{*} \\
& -\widehat{\mathcal{R}}_{Q}^{0}(\varepsilon)\left(b(\mathbf{D})^{*} L(\mathbf{D}) b(\mathbf{D})+\varepsilon^{2} b(\mathbf{D})^{*}(\overline{Q \Lambda})^{*}+\varepsilon^{2}(\overline{Q \Lambda}) b(\mathbf{D})\right) \widehat{\mathcal{R}}_{Q}^{0}(\varepsilon)+\widetilde{\mathcal{J}}^{\prime}
\end{aligned}
$$


and

$$
\begin{aligned}
&\left\|\check{\mathcal{J}}^{\prime}\right\|_{\mathfrak{G} \rightarrow \mathfrak{G}} \leq \mathcal{C}_{1}+2^{1 / 2} \alpha_{1}^{3 / 2} \alpha_{0}^{-5 / 2}\|g\|_{L_{\infty}^{3 / 2}}^{3 / 2}\left\|g^{-1}\right\|_{L_{\infty}}^{5 / 2}\|f\|_{L_{\infty}}^{2}\left\|f^{-1}\right\|_{L_{\infty}}^{2} r_{0}^{-2}=: \mathcal{C}_{1}^{\prime}, \\
&\left\|\widetilde{\mathcal{J}}^{\prime}\right\|_{\mathfrak{G} \rightarrow \mathfrak{G}} \leq \mathcal{C}_{1}+\mathfrak{C}=: \widetilde{\mathcal{C}}_{1}^{\prime},
\end{aligned}
$$

where $\mathcal{C}_{1}$ is the constant defined by (5.24), and $\mathfrak{C}$ is as in (7.15).

To replace $\Pi$ by $I$ in the first two terms in (7.2) and (7.6), it suffices to assume that the operator $[\Lambda]$ of multiplication by the matrix $\Lambda(\mathbf{x})$ (and then also the operator $\left[\Lambda_{Q}\right]$ of multiplication by $\Lambda_{Q}(\mathbf{x})$ ) maps $\mathfrak{G}_{*}^{1}$ into $\mathfrak{G}$ continuously, i.e., Condition 6.6 is satisfied. Recall that Condition 6.6 is ensured by the stronger Condition 6.10 .

The following theorem is a direct consequence of Theorem 7.4 and estimate (7.10).

Theorem 7.5. Under the assumptions of Theorem 7.4, suppose that either Condition 6.6 or (the stronger) Condition 6.10 is satisfied. Then for $\varepsilon>0$ we have

$$
\begin{aligned}
\left(\widehat{\mathcal{A}}+\varepsilon^{2} Q\right)^{-1}= & \widehat{\mathcal{R}}_{Q}^{0}(\varepsilon)+\Lambda_{Q} b(\mathbf{D}) \widehat{\mathcal{R}}_{Q}^{0}(\varepsilon)+\widehat{\mathcal{R}}_{Q}^{0}(\varepsilon) b(\mathbf{D})^{*}\left(\Lambda_{Q}\right)^{*} \\
& -\widehat{\mathcal{R}}_{Q}^{0}(\varepsilon) b(\mathbf{D})^{*} L_{Q}(\mathbf{D}) b(\mathbf{D}) \widehat{\mathcal{R}}_{Q}^{0}(\varepsilon)+\check{\mathcal{J}}^{\prime \prime} \\
\left(\widehat{\mathcal{A}}+\varepsilon^{2} Q\right)^{-1}= & \widehat{\mathcal{R}}_{Q}^{0}(\varepsilon)+\Lambda b(\mathbf{D}) \widehat{\mathcal{R}}_{Q}^{0}(\varepsilon)+\widehat{\mathcal{R}}_{Q}^{0}(\varepsilon) b(\mathbf{D})^{*} \Lambda^{*} \\
& -\widehat{\mathcal{R}}_{Q}^{0}(\varepsilon)\left(b(\mathbf{D})^{*} L(\mathbf{D}) b(\mathbf{D})+\varepsilon^{2} b(\mathbf{D})^{*}(\overline{Q \Lambda})^{*}+\varepsilon^{2}(\overline{Q \Lambda}) b(\mathbf{D})\right) \widehat{\mathcal{R}}_{Q}^{0}(\varepsilon) \\
& +\widetilde{\mathcal{J}}^{\prime \prime},
\end{aligned}
$$

and

$$
\begin{aligned}
&\left\|\check{\mathcal{J}}^{\prime \prime}\right\|_{\mathfrak{G} \rightarrow \mathfrak{G}} \leq \mathcal{C}_{1}^{\prime}+2 \alpha_{1}^{1 / 2} \alpha_{0}^{-1}\left\|g^{-1}\right\|_{L_{\infty}}\left(1+r_{0}^{-2}\right)^{1 / 2}\left\|\left[\Lambda_{Q}\right]\right\|_{\mathfrak{G}_{*}^{1} \rightarrow \mathfrak{G}}=: \mathcal{C}_{1}^{\prime \prime}, \\
&\left\|\widetilde{\mathcal{J}}^{\prime \prime}\right\|_{\mathfrak{G} \rightarrow \mathfrak{G}} \leq \widetilde{\mathcal{C}}_{1}^{\prime}+2 \alpha_{1}^{1 / 2} \alpha_{0}^{-1}\left\|g^{-1}\right\|_{L_{\infty}}\left(1+r_{0}^{-2}\right)^{1 / 2}\|[\Lambda]\|_{\mathfrak{G}_{*}^{1} \rightarrow \mathfrak{G}}=: \widetilde{\mathcal{C}}_{1}^{\prime \prime} .
\end{aligned}
$$

Here $\mathcal{C}_{1}^{\prime}$ is the constant defined by (7.16), and $\widetilde{\mathcal{C}}_{1}^{\prime}$ is the constant defined by (7.17).

Remark 7.6. By (5.4) and (7.14), we have

$$
\left\|\left[\Lambda_{Q}\right]\right\|_{\mathfrak{G}_{*}^{1} \rightarrow \mathfrak{G}} \leq\|[\Lambda]\|_{\mathfrak{G}_{*}^{1} \rightarrow \mathfrak{G}}+m^{1 / 2}\left(2 r_{0}\right)^{-1} \alpha_{0}^{-1 / 2}\|g\|_{L_{\infty}}^{1 / 2}\left\|g^{-1}\right\|_{L_{\infty}}^{1 / 2}\|Q\|_{L_{\infty}}\left\|Q^{-1}\right\|_{L_{\infty}} .
$$

This shows that the constants $\mathcal{C}_{1}^{\prime \prime}$ and $\widetilde{\mathcal{C}}_{1}^{\prime \prime}$ depend on $\|g\|_{L_{\infty}},\left\|g^{-1}\right\|_{L_{\infty}},\|Q\|_{L_{\infty}},\left\|Q^{-1}\right\|_{L_{\infty}}$,

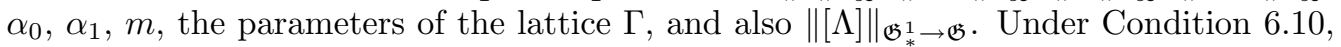
these constants depend only on $\|g\|_{L_{\infty}},\left\|g^{-1}\right\|_{L_{\infty}},\|Q\|_{L_{\infty}},\left\|Q^{-1}\right\|_{L_{\infty}}, \alpha_{0}, \alpha_{1}, m, n, d$, and the parameters of $\Gamma$.

Under the assumptions of Proposition 7.3, Condition 6.10 is valid automatically, so that in (7.4), $\Pi$ may be replaced by $I$. The following statement is true.

Proposition 7.7. Under the assumptions of Proposition 7.3, for $\varepsilon>0$ we have

$$
\left(\widehat{\mathcal{A}}+\varepsilon^{2} Q\right)^{-1}=\widehat{\mathcal{R}}_{Q}^{0}(\varepsilon)+\Lambda \mathbf{D} \widehat{\mathcal{R}}_{Q}^{0}(\varepsilon)+\widehat{\mathcal{R}}_{Q}^{0}(\varepsilon) \mathbf{D}^{*} \Lambda^{*}+\check{\mathcal{J}}^{\times},
$$

where $\check{\mathcal{J}}^{\times}$satisfies the estimate

$$
\left\|\check{\mathcal{J}}^{\times}\right\|_{\mathfrak{G} \rightarrow \mathfrak{G}} \leq \mathcal{C}_{1}+2\left\|g^{-1}\right\|_{L_{\infty}}\left(1+r_{0}^{-2}\right)^{1 / 2}\|[\Lambda]\|_{\mathfrak{G}_{*}^{1} \rightarrow \mathfrak{G}}=\mathcal{C}_{1}^{\times} .
$$

The constant $\mathcal{C}_{1}^{\times}$depends on $\|g\|_{L_{\infty}},\left\|g^{-1}\right\|_{L_{\infty}},\|Q\|_{L_{\infty}},\left\|Q^{-1}\right\|_{L_{\infty}}, d$, and the parameters of the lattice.

In the case where $g^{0}=\underline{g}$, we have $L(\mathbf{D})=0$ (see Proposition 4.5). Moreover, Condition 6.10 is satisfied. Hence, Theorem 7.5 applies, and the term in (7.18) that involves $b(\mathbf{D})^{*} L(\mathbf{D}) b(\mathbf{D})$ vanishes. As a result, we obtain the following. 
Proposition 7.8. Under the assumptions of Theorem 7.4, suppose that conditions (3.10) are satisfied (i.e., $g^{0}=\underline{g}$ ). Then for $\varepsilon>0$ we have

$$
\begin{aligned}
\left(\widehat{\mathcal{A}}+\varepsilon^{2} Q\right)^{-1}= & \widehat{\mathcal{R}}_{Q}^{0}(\varepsilon)+\Lambda b(\mathbf{D}) \widehat{\mathcal{R}}_{Q}^{0}(\varepsilon)+\widehat{\mathcal{R}}_{Q}^{0}(\varepsilon) b(\mathbf{D})^{*} \Lambda^{*} \\
& -\widehat{\mathcal{R}}_{Q}^{0}(\varepsilon)\left(\varepsilon^{2} b(\mathbf{D})^{*}(\overline{Q \Lambda})^{*}+\varepsilon^{2}(\overline{Q \Lambda}) b(\mathbf{D})\right) \widehat{\mathcal{R}}_{Q}^{0}(\varepsilon)+\widetilde{\mathcal{J}}^{\prime \prime},
\end{aligned}
$$

where $\widetilde{\mathcal{J}}^{\prime \prime}$ satisfies $(7.20)$.

By using the identity

$$
\left(\mathcal{A}+\varepsilon^{2} I\right)^{-1}=f^{-1}\left(\widehat{\mathcal{A}}+\varepsilon^{2} Q\right)^{-1}\left(f^{*}\right)^{-1}
$$

(cf. (5.26)), analogs of the theorems and propositions of this section can be obtained for the resolvent $\left(\mathcal{A}+\varepsilon^{2} I\right)^{-1}$. Herewith, the operators in the representations are multiplied by $f^{-1}$ from the left and by $\left(f^{*}\right)^{-1}$ from the right, while the constants in the remainder estimates are multiplied by $\left\|f^{-1}\right\|_{L_{\infty}}^{2}$.

\section{Chapter 3. Homogenization with Corrector term FOR PERIODIC DIFFERENTIAL OPERATORS}

Now we proceed to homogenization problems for periodic DOs in $L_{2}\left(\mathbb{R}^{d} ; \mathbb{C}^{n}\right)$ in the small period limit. If $\phi$ is a measurable $\Gamma$-periodic function on $\mathbb{R}^{d}$, we denote $\phi^{\varepsilon}(\mathbf{x})=$ $\phi\left(\varepsilon^{-1} \mathbf{x}\right)$ and put

$$
\widehat{\mathcal{A}}_{\varepsilon}=\widehat{\mathcal{A}}\left(g^{\varepsilon}\right), \quad \mathcal{A}_{\varepsilon}=\mathcal{A}\left(g^{\varepsilon}, f^{\varepsilon}\right) .
$$

In $\S 8$ we study approximation of the resolvent $\left(\widehat{\mathcal{A}}_{\varepsilon}+I\right)^{-1}$, and in $\S 9$ we study approximation of the generalized resolvent $\left(\widehat{\mathcal{A}}_{\varepsilon}+Q^{\varepsilon}\right)^{-1}$ and the resolvent $\left(\mathcal{A}_{\varepsilon}+I\right)^{-1}$. We take correctors into account and find approximations in terms of homogenized characteristics. In the $L_{2}$-operator norm, the resulting error term is of order $\varepsilon^{2}$. In similar results on the approximation without corrector, obtained in [BSu2], the error term is of order $\varepsilon$ (in the operator norm).

The theorems of this chapter ( $\S \S 8$ and 9) are the central results of the paper. At the same time, they follow from the corresponding results of $\S 6$ and $\S 7$ by a simple scaling transformation. The statements about homogenization for $\left(\mathcal{A}_{\varepsilon}+I\right)^{-1}$ follow from the results for the generalized resolvent $\left(\widehat{\mathcal{A}}_{\varepsilon}+Q^{\varepsilon}\right)^{-1}$ by an elementary recalculation.

\section{§8. Approximation of the Resolvent $\left(\widehat{\mathcal{A}}_{\varepsilon}+I\right)^{-1}$}

8.1. Setting of the problem. In the space $\mathfrak{G}=L_{2}\left(\mathbb{R}^{d} ; \mathbb{C}^{n}\right)$, we consider the family of operators

$$
\widehat{\mathcal{A}}_{\varepsilon}=\widehat{\mathcal{A}}\left(g^{\varepsilon}\right)=b(\mathbf{D})^{*} g^{\varepsilon}(\mathbf{x}) b(\mathbf{D}), \quad \varepsilon>0 .
$$

The matrix of coefficients $g^{\varepsilon}(\mathbf{x})$ of the operator (8.1) is rapidly oscillating as $\varepsilon \rightarrow 0$. One of the main problems of homogenization theory (as applied to the operator (8.1)) is to study the behavior of the solution $\mathbf{u}_{\varepsilon}$ of the equation

$$
\widehat{\mathcal{A}}_{\varepsilon} \mathbf{u}_{\varepsilon}+\mathbf{u}_{\varepsilon}=\mathbf{F}, \quad \mathbf{F} \in \mathfrak{G},
$$

as $\varepsilon \rightarrow 0$. In other words, the behavior of the resolvent $\left(\widehat{\mathcal{A}}_{\varepsilon}+I\right)^{-1}$ must be investigated. Let $g^{0}$ be the effective matrix, and let $\widehat{\mathcal{A}}^{0}=\widehat{\mathcal{A}}\left(g^{0}\right)$ be the effective operator with constant coefficients. Along with equation (8.2), we consider the "homogenized" equation

$$
\widehat{\mathcal{A}}^{0} \mathbf{u}_{0}+\mathbf{u}_{0}=\mathbf{F} \text {. }
$$

The problem studied in homogenization theory is that of convergence (in some sense) as $\varepsilon \rightarrow 0$ of the solution of equation (8.2) to the solution of equation (8.3). 
In [BSu1, BSu2, this problem was solved in terms of convergence of resolvents. It was shown that

$$
\left\|\left(\widehat{\mathcal{A}}_{\varepsilon}+I\right)^{-1}-\left(\widehat{\mathcal{A}}^{0}+I\right)^{-1}\right\|_{\mathfrak{G} \rightarrow \mathfrak{G}} \leq C \varepsilon
$$

where the constant $C$ is controlled explicitly. Now our goal is to find an approximation with corrector for $\left(\widehat{\mathcal{A}}_{\varepsilon}+I\right)^{-1}$, with the remainder estimate of order $\varepsilon^{2}$. For this, it suffices to make the scale transformation in the statements of $\S 6$.

8.2. The main result. Let $T_{\varepsilon}$ be the scaling transformation: $\left(T_{\varepsilon} \mathbf{u}\right)(\mathbf{y})=\varepsilon^{d / 2} \mathbf{u}(\varepsilon \mathbf{y})$, $\mathbf{y} \in \mathbb{R}^{d}$. The operator $T_{\varepsilon}$ is unitary in $\mathfrak{G}$. Let $\widehat{\mathcal{A}}=\widehat{\mathcal{A}}(g)$. Then $\widehat{\mathcal{A}}_{\varepsilon}=\varepsilon^{-2} T_{\varepsilon}^{*} \widehat{\mathcal{A}} T_{\varepsilon}$, whence

$$
\left(\widehat{\mathcal{A}}_{\varepsilon}+I\right)^{-1}=\varepsilon^{2} T_{\varepsilon}^{*}\left(\widehat{\mathcal{A}}+\varepsilon^{2} I\right)^{-1} T_{\varepsilon} .
$$

If the matrix $g=g^{0}$ is constant, then (8.4) turns into a simpler relation

$$
\left(\widehat{\mathcal{A}}^{0}+I\right)^{-1}=\varepsilon^{2} T_{\varepsilon}^{*}\left(\widehat{\mathcal{A}}^{0}+\varepsilon^{2} I\right)^{-1} T_{\varepsilon} .
$$

Obviously,

$$
\begin{gathered}
T_{\varepsilon}^{*}[\Lambda] T_{\varepsilon}=\left[\Lambda^{\varepsilon}\right], \quad T_{\varepsilon}^{*} b(\mathbf{D}) T_{\varepsilon}=\varepsilon b(\mathbf{D}), \\
\varepsilon^{-2} T_{\varepsilon}^{*} b(\mathbf{D})^{*} L(\mathbf{D}) b(\mathbf{D}) T_{\varepsilon}=\varepsilon b(\mathbf{D})^{*} L(\mathbf{D}) b(\mathbf{D}) .
\end{gathered}
$$

Next, we denote

$$
\Pi_{\varepsilon}:=T_{\varepsilon}^{*} \Pi T_{\varepsilon} .
$$

Let $\chi_{\widetilde{\Omega} / \varepsilon}$ be the indicator of the set $\varepsilon^{-1} \widetilde{\Omega}$. Then $\Pi_{\varepsilon}$ is the pseudodifferential operator with the symbol $\chi_{\widetilde{\Omega} / \varepsilon}(\xi)$, i.e.,

$$
\Pi_{\varepsilon}=\mathcal{F}^{*}\left[\chi_{\widetilde{\Omega} / \varepsilon}(\cdot)\right] \mathcal{F} .
$$

We employ Theorem 6.4. Multiplying (6.20) by $\varepsilon^{2} T_{\varepsilon}^{*}$ from the left and by $T_{\varepsilon}$ from the right, and using (8.4)-(8.7), we obtain

$$
\begin{aligned}
\left(\widehat{\mathcal{A}}_{\varepsilon}+I\right)^{-1}= & \widehat{\mathcal{R}}^{0}+\varepsilon \Lambda^{\varepsilon} b(\mathbf{D}) \Pi_{\varepsilon} \widehat{\mathcal{R}}^{0}+\varepsilon \widehat{\mathcal{R}}^{0}\left(\Lambda^{\varepsilon} b(\mathbf{D}) \Pi_{\varepsilon}\right)^{*} \\
& -\varepsilon \widehat{\mathcal{R}}^{0} b(\mathbf{D})^{*} L(\mathbf{D}) b(\mathbf{D}) \widehat{\mathcal{R}}^{0}+\varepsilon^{2} \widehat{\mathcal{I}}^{\prime}(\varepsilon),
\end{aligned}
$$

where $\widehat{\mathcal{R}}^{0}=\left(\widehat{\mathcal{A}}^{0}+I\right)^{-1}$ and $\widehat{\mathcal{I}}^{\prime}(\varepsilon)=T_{\varepsilon}^{*} \widehat{\mathcal{J}}^{\prime} T_{\varepsilon}$. Thus, we arrive at the following statement.

Theorem 8.1. Let $\widehat{\mathcal{A}}_{\varepsilon}=\widehat{\mathcal{A}}\left(g^{\varepsilon}\right)$, and let $\widehat{\mathcal{A}}^{0}=\widehat{\mathcal{A}}\left(g^{0}\right)$ be the effective operator. Let $\Lambda(\mathbf{x})$ be the periodic $(n \times m)$-matrix-valued function with the columns $\mathbf{v}_{j}(\mathbf{x}), j=1, \ldots, m$, where $\mathbf{v}_{j}$ is the solution of the equation (3.4) with $\mathbf{C}=\mathbf{e}_{j}$, and let $\Lambda^{\varepsilon}(\mathbf{x})=\Lambda\left(\varepsilon^{-1} \mathbf{x}\right)$. Let $L(\mathbf{k})=t L(\boldsymbol{\theta})$ be defined in accordance with (3.5) and (4.8), let $L(\mathbf{D})$ be the first-order DO with the symbol $L(\mathbf{k})$, and let $\Pi_{\varepsilon}$ be the pseudodifferential operator (8.8). Then for $\varepsilon>0$ the representation (8.9) is valid, and

$$
\left\|\widehat{\mathcal{I}}^{\prime}(\varepsilon)\right\|_{\mathfrak{G} \rightarrow \mathfrak{G}} \leq \widehat{\mathcal{C}}_{1}^{\prime} .
$$

The constant $\widehat{\mathcal{C}}_{1}^{\prime}$ is defined by (6.21) and depends only on $\|g\|_{L_{\infty}},\left\|g^{-1}\right\|_{L_{\infty}}, \alpha_{0}, \alpha_{1}$, and the parameters of the lattice.

The operator coefficient of $\varepsilon$ on the right-hand side of (8.9) is called the corrector. The corrector

$$
K(\varepsilon)=\Lambda^{\varepsilon} b(\mathbf{D}) \Pi_{\varepsilon} \widehat{\mathcal{R}}^{0}+\widehat{\mathcal{R}}^{0}\left(\Lambda^{\varepsilon} b(\mathbf{D}) \Pi_{\varepsilon}\right)^{*}-\widehat{\mathcal{R}}^{0} b(\mathbf{D})^{*} L(\mathbf{D}) b(\mathbf{D}) \widehat{\mathcal{R}}^{0}
$$

involves three summands. The first two operators in (8.11) are mutually adjoint. It is essential that they contain the rapidly oscillating factor $\Lambda^{\varepsilon}$. The third term differs from the first two terms in principle, because this term is a pseudodifferential operator (of order $(-1)$ ) with a symbol independent of $\mathbf{x}$ and $\varepsilon$. In particular, this term does not involve $\Pi_{\varepsilon}$. 
8.3. "Elimination" of $\Pi_{\varepsilon}$. Now we consider conditions under which $\Pi_{\varepsilon}$ may be replaced by $I$ in the first two terms of the corrector. The following statement is deduced from Theorem 6.12 by scaling.

Theorem 8.2. Under the assumptions of Theorem 8.1, suppose that either Condition 6.6 or (the stronger) Condition 6.10 is satisfied. Then for $\varepsilon>0$ we have

$$
\begin{aligned}
\left(\widehat{\mathcal{A}}_{\varepsilon}+I\right)^{-1}= & \widehat{\mathcal{R}}^{0}+\varepsilon \Lambda^{\varepsilon} b(\mathbf{D}) \widehat{\mathcal{R}}^{0}+\varepsilon \widehat{\mathcal{R}}^{0} b(\mathbf{D})^{*}\left(\Lambda^{\varepsilon}\right)^{*} \\
& -\varepsilon \widehat{\mathcal{R}}^{0} b(\mathbf{D})^{*} L(\mathbf{D}) b(\mathbf{D}) \widehat{\mathcal{R}}^{0}+\varepsilon^{2} \widehat{\mathcal{I}}^{\prime \prime}(\varepsilon),
\end{aligned}
$$

where

$$
\left\|\widehat{\mathcal{I}}^{\prime \prime}(\varepsilon)\right\|_{\mathfrak{G} \rightarrow \mathfrak{G}} \leq \widehat{\mathcal{C}}_{1}^{\prime \prime} .
$$

The constant $\widehat{\mathcal{C}_{1}^{\prime \prime}}$ is defined by (6.32) and depends on $\|g\|_{L_{\infty}},\left\|g^{-1}\right\|_{L_{\infty}}, \alpha_{0}, \alpha_{1}$, the pa-

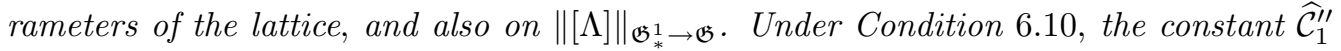
depends only on $\|g\|_{L_{\infty}},\left\|g^{-1}\right\|_{L_{\infty}}, \alpha_{0}, \alpha_{1}, m, n, d$, and the parameters of the lattice.

In (8.12), the corrector (to be denoted by $K^{\circ}(\varepsilon)$ ) is simpler than the corrector $K(\varepsilon)$ in $(8.11)$ :

$$
K^{\circ}(\varepsilon)=\Lambda^{\varepsilon} b(\mathbf{D}) \widehat{\mathcal{R}}^{0}+\widehat{\mathcal{R}}^{0} b(\mathbf{D})^{*}\left(\Lambda^{\varepsilon}\right)^{*}-\widehat{\mathcal{R}}^{0} b(\mathbf{D})^{*} L(\mathbf{D}) b(\mathbf{D}) \widehat{\mathcal{R}}^{0} .
$$

Observe that, under the assumptions of Theorem 8.2, the operator norm of the difference $K(\varepsilon)-K^{\circ}(\varepsilon)$ is $O(\varepsilon)$.

8.4. Particular cases. First of all, we distinguish the case where the corrector is equal to zero. The following theorem is deduced from Theorem 6.2 by scaling.

Theorem 8.3. Under the assumptions of Theorem 8.1, let $\Lambda(\mathbf{x}) b(\boldsymbol{\theta})=0$ for all $\boldsymbol{\theta} \in \mathbb{S}^{d-1}$, $\mathbf{x} \in \Omega$. Then

$$
\left\|\left(\widehat{\mathcal{A}}_{\varepsilon}+I\right)^{-1}-\left(\widehat{\mathcal{A}}^{0}+I\right)^{-1}\right\|_{\mathfrak{G} \rightarrow \mathfrak{G}} \leq \widehat{\mathcal{C}}_{2} \varepsilon^{2}, \quad \varepsilon>0,
$$

where the constant $\widehat{\mathcal{C}_{2}}$ is defined by (4.30) and depends on $\|g\|_{L_{\infty}},\left\|g^{-1}\right\|_{L_{\infty}}, \alpha_{0}, \alpha_{1}$, and the parameters of the lattice. In particular, (8.15) is true under conditions (3.9) (i.e., if $\left.g^{0}=\bar{g}\right)$.

Thus, under the assumptions of Theorem 8.3, the result of [BSu2] can be revised (only an estimate of order $C \varepsilon$ was obtained in [BSu2]).

Proposition 6.13 implies the following condition, ensuring that the third term of the corrector (8.14) is equal to zero.

Proposition 8.4. Under the assumptions of Theorem 8.1, suppose that either conditions (3.10) are satisfied (i.e., $g^{0}=\underline{g}$ ) or $\widehat{\mathcal{A}}=\mathbf{D}^{*} g(\mathbf{x}) \mathbf{D}$, where the matrix $g(\mathbf{x})$ has real entries. Then

$$
\left(\widehat{\mathcal{A}}_{\varepsilon}+I\right)^{-1}=\widehat{\mathcal{R}}^{0}+\varepsilon \Lambda^{\varepsilon} b(\mathbf{D}) \widehat{\mathcal{R}}^{0}+\varepsilon \widehat{\mathcal{R}}^{0}\left(\Lambda^{\varepsilon} b(\mathbf{D})\right)^{*}+\varepsilon^{2} \widehat{\mathcal{I}}^{\times}(\varepsilon), \quad \varepsilon>0,
$$

where

$$
\left\|\widehat{\mathcal{I}}^{\times}(\varepsilon)\right\|_{\mathfrak{G} \rightarrow \mathfrak{G}} \leq \widehat{\mathcal{C}}_{1}^{\times} .
$$

The constant $\widehat{\mathcal{C}}_{1}^{\times}$is defined by (4.29) and (6.33) and depends on $\|g\|_{L_{\infty}},\left\|g^{-1}\right\|_{L_{\infty}}, \alpha_{0}$, $\alpha_{1}, m, n, d$, and the parameters of the lattice. In particular, (8.16) and (8.17) are true if $m=n$. 
8.5. Discussion. For the operators of the form $\widehat{\mathcal{A}}_{\varepsilon}=b(\mathbf{D})^{*} g^{\varepsilon} b(\mathbf{D})$, Theorems 8.1 and 8.2 are the main results of the paper. Note that in Theorem 8.1 we did not need any smoothness assumptions on the matrix $g$. The same is true for Theorem 8.2 in the case where Condition 6.10 is satisfied. All this is related to the incorporation of two new terms in the corrector, as compared with the traditional corrector of homogenization theory. The traditional corrector corresponds to the first summand of the corrector $K^{\circ}(\varepsilon)$ (see (8.14)). Below we discuss the reasons for this difference and the meaning of it.

We did not study approximation of the solution of equation (8.2) in the norm of $\mathfrak{G}^{1}=H^{1}\left(\mathbb{R}^{d} ; \mathbb{C}^{n}\right)$. However, it can be expected that the following estimate is true:

$$
\left\|(\widehat{\mathcal{A}}+I)^{-1}-\left(\widehat{\mathcal{A}}^{0}+I\right)^{-1}-\varepsilon \mathcal{K}(\varepsilon)\right\|_{\mathfrak{G} \rightarrow \mathfrak{G}^{1}} \leq C \varepsilon,
$$

where $\mathcal{K}(\varepsilon)$ is the corrector (8.11) (under the conditions of Theorem 8.1), or even the corrector (8.14) (under more restrictive conditions). Until now, no estimates of the form (8.18) were known. Only very recently the paper Zh4 by Zhikov appeared. In that paper, by a method different from ours, estimate (8.18) was obtained for the acoustics operator $\widehat{\mathcal{A}}=\mathbf{D}^{*} g \mathbf{D}$; the role of $\mathcal{K}(\varepsilon)$ was played by the operator $K_{1}^{\circ}(\varepsilon)=\Lambda^{\varepsilon} \mathbf{D} \widehat{\mathcal{R}}^{0}$. In general, the "truncated" corrector $\Lambda^{\varepsilon} b(\mathbf{D}) \widehat{\mathcal{R}}^{0}$ is traditional for homogenization theory, where the usual goal is to approximate the solution $\mathbf{u}_{\varepsilon}$ in the $H^{1}$-norm, at least under some smoothness assumptions on $g$ and $\mathbf{F}$. At the same time, in order to obtain the representations (8.9), (8.10) or (8.12), (8.13), we need the full correctors (8.11) or (8.14). This can be explained as follows.

Let $\mathcal{K}_{j}(\varepsilon), j=1,2,3$, denote the corresponding summands of the corrector $\mathcal{K}(\varepsilon)$. The operator $\mathcal{K}_{3}$ in (8.11) and (8.14) is one and the same; it does not depend on $\varepsilon$. Obviously, $\mathcal{K}_{3}$ maps $\mathfrak{G}$ into $\mathfrak{G}^{1}$ continuously. Therefore, if (8.18) is true, then the term $\varepsilon \mathcal{K}_{3}$ can be included in the error term. The situation with the summand $\mathcal{K}_{2}(\varepsilon)$ is different. It is easily seen that, under the conditions of Theorem 8.1, i.e., for the corrector (8.11), we have

$$
\sup _{\varepsilon>0}\left\|\mathcal{K}_{2}(\varepsilon)\right\|_{\mathfrak{G} \rightarrow \mathfrak{G}^{1}}<\infty .
$$

Then the term $\varepsilon \mathcal{K}_{2}(\varepsilon)$ can also be included in the error term. If $\mathcal{K}(\varepsilon)=K^{\circ}(\varepsilon)$, estimate (8.19) is fulfilled as long as $\Lambda \in L_{\infty}(\Omega)$. The latter is true if $g^{0}=\underline{g}$, or if we deal with the operator $\widehat{\mathcal{A}}=\mathbf{D}^{*} g \mathbf{D}$, where the matrix $g$ has real entries, or with the operators $\widehat{\mathcal{A}}$ of general type but under appropriate smoothness assumptions on the matrix $g$. Finally, we recall that, under the conditions of Theorem 8.2, the operator $K_{1}^{\circ}(\varepsilon)$ does not involve the factor $\Pi_{\varepsilon}$.

The above discussion explains why, in order to obtain estimates of the form (8.18), it makes sense to use the "truncated" corrector $K_{1}^{\circ}(\varepsilon)=\Lambda^{\varepsilon} b(\mathbf{D}) \widehat{\mathcal{R}}^{0}$. This was done in [Zh4] for the operator $\widehat{\mathcal{A}}=\mathbf{D}^{*} g \mathbf{D}$. However, if an estimate of the form (8.18) has already been obtained with the truncated corrector, then it remains valid also with the full corrector. The problem of establishing estimate (8.18) is not related to this difference. Below we use the opportunity to vary the form of the corrector (see Proposition 10.4 concerning interpolation).

8.6. About the weak operator limit of the corrector. In this subsection we keep the notation of Subsection 8.5. We calculate the weak operator limit of the corrector $K(\varepsilon)$ as $\varepsilon \rightarrow 0$. The term

$$
K_{3}=-\widehat{\mathcal{R}}^{0} b(\mathbf{D})^{*} L(\mathbf{D}) b(\mathbf{D}) \widehat{\mathcal{R}}^{0}
$$

does not depend on $\varepsilon$ at all. Next, the following statement is true. 
Proposition 8.5. Under the assumptions of Theorem 8.1, the weak $(\mathfrak{G} \rightarrow \mathfrak{G})$-limit of the operators $K_{j}(\varepsilon), j=1,2$, is equal to zero.

Obviously, it suffices to consider the operator

$$
K_{1}(\varepsilon)=\Lambda^{\varepsilon} b(\mathbf{D}) \Pi_{\varepsilon} \widehat{\mathcal{R}}^{0} .
$$

We start with the following lemma, which will be proved later.

Lemma 8.6. Suppose that a $\Gamma$-periodic function $Y$ belongs to the class $L_{2, \operatorname{loc}}\left(\mathbb{R}^{d}\right)$. Let $\Pi_{\varepsilon}$ be the pseudodifferential operator $(8.8)$. Then the weak $(\mathfrak{G} \rightarrow \mathfrak{G})$-limit of the operators $\left[Y^{\varepsilon}\right] \Pi_{\varepsilon}$ is given by the formula

$$
\mathrm{w}-\lim _{\varepsilon \rightarrow 0}\left[Y^{\varepsilon}\right] \Pi_{\varepsilon}=\bar{Y} .
$$

Proof of Proposition 8.5. Now the proof is obvious. The operator $b(\mathbf{D}) \widehat{\mathcal{R}}^{0}$ in $\mathfrak{G}$ is continuous, and

$$
\mathrm{w}-\lim _{\varepsilon \rightarrow 0} \Lambda^{\varepsilon} \Pi_{\varepsilon}=0
$$

by (8.21). Here we have used the fact that $\bar{\Lambda}=0$.

Proposition 8.7. Under the assumptions of Theorem 8.1, we have

$$
\underset{\varepsilon \rightarrow 0}{\mathrm{w}-\lim } K(\varepsilon)=K_{3} .
$$

Under the assumptions of Theorem 8.2, we have

$$
\underset{\varepsilon \rightarrow 0}{\mathrm{w}-\lim _{\varepsilon \rightarrow 0}} K^{\circ}(\varepsilon)=K_{3} .
$$

Proof. Formula (8.22) is obvious now. Formula (8.23) is its consequence, because, as was already mentioned in Subsection 8.3, under the conditions of Theorem 8.2 we have $\left\|K(\varepsilon)-K^{\circ}(\varepsilon)\right\|_{\mathfrak{G} \rightarrow \mathfrak{G}}=O(\varepsilon)$.

Relations (8.22) and (8.23) show that, in a sense, the term $K_{3}$ (if it is nonzero) is the leading term of the corrector. This term is independent of $\varepsilon$, while the other two terms oscillate and their "mean value" is equal to zero.

Relation (8.22) allows us to deduce an interesting consequence from the representation (8.9). As the value of the operator-valued function $\left(\widehat{\mathcal{A}}_{\varepsilon}+I\right)^{-1}$ for $\varepsilon=0$ we take $\left(\widehat{\mathcal{A}}^{0}+I\right)^{-1}$. The resulting function is continuous from the right at $\varepsilon=0$ in the operator $(\mathfrak{G} \rightarrow \mathfrak{G})$ norm. We write (8.9) as

$$
\varepsilon^{-1}\left(\left(\widehat{\mathcal{A}}_{\varepsilon}+I\right)^{-1}-\left(\widehat{\mathcal{A}}^{0}+I\right)^{-1}\right)=K(\varepsilon)+\varepsilon \widehat{\mathcal{I}}^{\prime}(\varepsilon) .
$$

Then, as $\varepsilon \rightarrow 0,(8.10)$ and (8.22) yield the following statement.

Proposition 8.8. Under the assumptions of Theorem 8.1, the operator-valued function $\left(\widehat{\mathcal{A}}_{\varepsilon}+I\right)^{-1}$ admits a weak derivative with respect to $\varepsilon$ at the point $\varepsilon=0$. This derivative is equal to the operator $K_{3}$ defined by (8.20).

We observe that, at the initial (operator-theoretic) level, the term $K_{3}$ in the corrector is generated by the operator $N$ defined by (1.13) and involved in the representation (1.23) for the resolvent $\left(A(t)+\varepsilon^{2} I\right)^{-1}$. On the other hand, by (1.15), the operator $N$ itself is the sum of two terms: $N=N_{0}+N_{*}$, where $N_{0}$ and $N_{*}$ are defined by (1.16) and (1.17), respectively. The operators $N_{0}$ and $N_{*}$ are of different origin and have different properties. We have already distinguished the case (see, e.g., Proposition 4.2 and Corollary 4.3) where the operator $N_{0}$ makes no contribution. On the other hand (see Subsection 1.4), $N_{*}=0$ for $n=1$. In other words, the operator $N_{*}$ can appear only in vector problems, while the operator $N_{0}$ mainly appears in the case of operators with 
complex-valued coefficients. Examples where the first case occurs can be found below in Subsections 13.2 and 14.6, and the second case is illustrated in Subsection 10.4.

It remains to prove Lemma 8.6.

Proof of Lemma 8.6. First of all, we check that

$$
\sup _{\varepsilon>0}\left\|Y^{\varepsilon} \Pi_{\varepsilon}\right\|_{\mathfrak{G} \rightarrow \mathfrak{G}}<\infty .
$$

Since $Y^{\varepsilon} \Pi_{\varepsilon}=T_{\varepsilon}^{*}(Y \Pi) T_{\varepsilon}$ (see (8.6), (8.7)), it suffices to show that the operator $Y \Pi$ is bounded on $\mathfrak{G}$. Since $\Pi$ is smoothing infinitely, we have

$$
\|\Pi \mathbf{u}\|_{H^{s}\left(\mathbb{R}^{d} ; \mathbb{C}^{n}\right)}^{2} \leq c_{s}\|\mathbf{u}\|_{\mathfrak{G}}^{2}, \quad \mathbf{u} \in \mathfrak{G}, \quad \text { for all } s>0 .
$$

Let $s$ be an integer, and let $2 s>d$. For any $\mathbf{a} \in \Gamma$ we have

$$
\int_{\Omega+\mathbf{a}}|Y(\mathbf{x}) \Pi \mathbf{u}|^{2} d \mathbf{x} \leq\|Y\|_{L_{2}(\Omega)}^{2}\|\Pi \mathbf{u}\|_{L_{\infty}(\Omega+\mathbf{a})}^{2} \leq c^{\circ}\|Y\|_{L_{2}(\Omega)}^{2}\|\Pi \mathbf{u}\|_{H^{s}\left(\Omega+\mathbf{a}, \mathbb{C}^{n}\right)}^{2},
$$

where $c^{\circ}=c^{\circ}(s, d, \Gamma)$ is the constant occurring in the theorem on the embedding of $H^{s}(\Omega)$ in $L_{\infty}(\Omega)$. Summing over $\mathbf{a} \in \Gamma$, we obtain

$$
\|Y \Pi \mathbf{u}\|_{\mathfrak{G}}^{2} \leq c^{\circ}\|Y\|_{L_{2}(\Omega)}^{2}\|\Pi \mathbf{u}\|_{H^{s}\left(\mathbb{R}^{d}, \mathbb{C}^{n}\right)}^{2} \leq c^{\circ} c_{s}\|Y\|_{L_{2}(\Omega)}^{2}\|\mathbf{u}\|_{\mathfrak{G}}^{2},
$$

which proves (8.24). Now it suffices to calculate the limit of $\left(Y^{\varepsilon} \prod_{\varepsilon} \mathbf{u}, \mathbf{v}\right)_{\mathfrak{G}}$ as $\varepsilon \rightarrow 0$ at the vector-valued functions $\mathbf{v} \in C_{0}^{\infty}\left(\mathbb{R}^{d} ; \mathbb{C}^{n}\right)$ and for the functions $\mathbf{u}$ such that $\mathcal{F} \mathbf{u} \in$ $C_{0}^{\infty}\left(\mathbb{R}^{d} ; \mathbb{C}^{n}\right)$. But in this case $\Pi_{\varepsilon} \mathbf{u}=\mathbf{u}$ for sufficiently small $\varepsilon>0$, and it remains to refer to the mean value property (see, e.g., [ZhKO]), by which $Y^{\varepsilon}$ converges to $\bar{Y}$ weakly in $L_{2, \text { loc }}\left(\mathbb{R}^{d} ; \mathbb{C}^{n}\right)$.

\section{§9. Approximation for $\left(\widehat{\mathcal{A}}_{\varepsilon}+Q^{\varepsilon}\right)^{-1}$ And $\left(\mathcal{A}_{\varepsilon}+I\right)^{-1}$}

9.1. Now, suppose that, along with the operator (8.1), a $\Gamma$-periodic positive $(n \times n)$ matrix-valued function $Q(\mathbf{x})$ is given. Let $Q, Q^{-1} \in L_{\infty}$. Putting $Q^{\varepsilon}(\mathbf{x})=Q\left(\varepsilon^{-1} \mathbf{x}\right)$, we study the behavior of the generalized resolvent $\left(\widehat{\mathcal{A}}_{\varepsilon}+Q^{\varepsilon}\right)^{-1}$ as $\varepsilon \rightarrow 0$.

This question is closely related to the problem of approximation for the resolvent $\left(\mathcal{A}_{\varepsilon}+I\right)^{-1}$ of the family

$$
\mathcal{A}_{\varepsilon}=\mathcal{A}\left(g^{\varepsilon}, f^{\varepsilon}\right)=\left(f^{\varepsilon}(\mathbf{x})\right)^{*} b(\mathbf{D})^{*} g^{\varepsilon}(\mathbf{x}) b(\mathbf{D}) f^{\varepsilon}(\mathbf{x}) .
$$

We put $Q(\mathbf{x})=\left(f(\mathbf{x}) f(\mathbf{x})^{*}\right)^{-1}$, obtaining the identity

$$
\left(\mathcal{A}_{\varepsilon}+I\right)^{-1}=\left(f^{\varepsilon}\right)^{-1}\left(\widehat{\mathcal{A}}_{\varepsilon}+Q^{\varepsilon}\right)^{-1}\left(\left(f^{\varepsilon}\right)^{*}\right)^{-1} .
$$

The results concerning approximation of the generalized resolvent $\left(\widehat{\mathcal{A}}_{\varepsilon}+Q^{\varepsilon}\right)^{-1}$ follow from the results of $\S 7$ by scaling. By (9.2), they directly imply results on the approximation of the resolvent $\left(\mathcal{A}_{\varepsilon}+I\right)^{-1}$. We combine the corresponding statements "pairwise".

We use identities similar to (8.4) and (8.5):

$$
\begin{aligned}
\left(\widehat{\mathcal{A}}_{\varepsilon}+Q^{\varepsilon}\right)^{-1} & =\varepsilon^{2} T_{\varepsilon}^{*}\left(\widehat{\mathcal{A}}+\varepsilon^{2} Q\right)^{-1} T_{\varepsilon}, \\
\left(\widehat{\mathcal{A}}^{0}+\bar{Q}\right)^{-1} & =\varepsilon^{2} T_{\varepsilon}^{*}\left(\widehat{\mathcal{A}}^{0}+\varepsilon^{2} \bar{Q}\right)^{-1} T_{\varepsilon} .
\end{aligned}
$$

The following result is a consequence of Theorem 7.4 and identities (9.3) and (9.4) (cf. the proof of Theorem 8.1).

Theorem 9.1. 1) Let $\widehat{\mathcal{A}_{\varepsilon}}=\widehat{\mathcal{A}}\left(g^{\varepsilon}\right)$, and let $\widehat{\mathcal{A}}^{0}=\widehat{\mathcal{A}}\left(g^{0}\right)$ be the effective operator. Suppose that $Q(\mathbf{x})$ is a periodic positive $(n \times n)$-matrix-valued function such that $Q, Q^{-1} \in L_{\infty}$. Let $\bar{Q}=|\Omega|^{-1} \int_{\Omega} Q(\mathbf{x}) d \mathbf{x}$. Suppose that $\Lambda(\mathbf{x})$ is the periodic $(n \times m)$-matrix-valued function with the columns $\mathbf{v}_{j}(\mathbf{x}), j=1, \ldots, m$, where $\mathbf{v}_{j}$ is the solution of the problem (3.4) with $\mathbf{C}=\mathbf{e}_{j}$. Let $\Lambda_{Q}(\mathbf{x})$ be the matrix-valued function defined by (5.4). Suppose 
that the matrix-valued functions $L(\mathbf{k})=t L(\boldsymbol{\theta})$ and $L_{Q}(\mathbf{k})=t L_{Q}(\boldsymbol{\theta})$ are defined by (3.5), (4.8) and (5.12). Let $L(\mathbf{D}), L_{Q}(\mathbf{D})$ be the first-order DOs with the symbols $L(\mathbf{k})$ and $L_{Q}(\mathbf{k})$, respectively, and let $\Pi_{\varepsilon}$ be the pseudodifferential operator (8.8). Then for $\varepsilon>0$ we have

$$
\begin{aligned}
\left(\widehat{\mathcal{A}}_{\varepsilon}+Q^{\varepsilon}\right)^{-1}= & \widehat{\mathcal{R}}_{Q}^{0}+\varepsilon K_{Q}(\varepsilon)+\varepsilon^{2} \check{\mathcal{I}}^{\prime}(\varepsilon), \\
K_{Q}(\varepsilon)= & \Lambda_{Q}^{\varepsilon} b(\mathbf{D}) \Pi_{\varepsilon} \widehat{\mathcal{R}}_{Q}^{0}+\widehat{\mathcal{R}}_{Q}^{0}\left(\Lambda_{Q}^{\varepsilon} b(\mathbf{D}) \Pi_{\varepsilon}\right)^{*}-\widehat{\mathcal{R}}_{Q}^{0} b(\mathbf{D})^{*} L_{Q}(\mathbf{D}) b(\mathbf{D}) \widehat{\mathcal{R}}_{Q}^{0}, \\
\left(\widehat{\mathcal{A}}_{\varepsilon}+Q^{\varepsilon}\right)^{-1}= & \widehat{\mathcal{R}}_{Q}^{0}+\varepsilon \widetilde{K}_{Q}(\varepsilon)+\varepsilon^{2} \widetilde{\mathcal{I}}^{\prime}(\varepsilon), \\
\widetilde{K}_{Q}(\varepsilon)= & \Lambda^{\varepsilon} b(\mathbf{D}) \Pi_{\varepsilon} \widehat{\mathcal{R}}_{Q}^{0}+\widehat{\mathcal{R}}_{Q}^{0}\left(\Lambda^{\varepsilon} b(\mathbf{D}) \Pi_{\varepsilon}\right)^{*} \\
& -\widehat{\mathcal{R}}_{Q}^{0}\left(b(\mathbf{D})^{*} L(\mathbf{D}) b(\mathbf{D})+b(\mathbf{D})^{*}(\overline{Q \Lambda})^{*}+(\overline{Q \Lambda}) b(\mathbf{D})\right) \widehat{\mathcal{R}}_{Q}^{0},
\end{aligned}
$$

where $\widehat{\mathcal{R}}_{Q}^{0}=\left(\widehat{\mathcal{A}}^{0}+\bar{Q}\right)^{-1}$, and

$$
\left\|\check{\mathcal{I}}^{\prime}(\varepsilon)\right\|_{\mathfrak{G} \rightarrow \mathfrak{G}} \leq \mathcal{C}_{1}^{\prime}, \quad\left\|\widetilde{\mathcal{I}}^{\prime}(\varepsilon)\right\|_{\mathfrak{G} \rightarrow \mathfrak{G}} \leq \widetilde{\mathcal{C}}_{1}^{\prime},
$$

where the constants $\mathcal{C}_{1}^{\prime}, \widetilde{\mathcal{C}}_{1}^{\prime}$ are defined by (7.16), (7.17) and depend on $\|g\|_{L_{\infty}},\left\|g^{-1}\right\|_{L_{\infty}}$, $\|Q\|_{L_{\infty}},\left\|Q^{-1}\right\|_{L_{\infty}}, \alpha_{0}, \alpha_{1}, m$, and the parameters of the lattice $\Gamma$.

2) Let $\mathcal{A}_{\varepsilon}=\mathcal{A}\left(g^{\varepsilon}, f^{\varepsilon}\right)$ be the operator (9.1), and suppose that the conditions of statement 1 ) are satisfied with $Q(\mathbf{x})=\left(f(\mathbf{x}) f(\mathbf{x})^{*}\right)^{-1}$. Then for $\varepsilon>0$ we have

$$
\begin{aligned}
& \left(\mathcal{A}_{\varepsilon}+I\right)^{-1}=\left(f^{\varepsilon}\right)^{-1}\left(\widehat{\mathcal{R}}_{Q}^{0}+\varepsilon K_{Q}(\varepsilon)\right)\left(\left(f^{\varepsilon}\right)^{*}\right)^{-1}+\varepsilon^{2} \mathcal{Y}^{\prime}(\varepsilon), \\
& \left(\mathcal{A}_{\varepsilon}+I\right)^{-1}=\left(f^{\varepsilon}\right)^{-1}\left(\widehat{\mathcal{R}}_{Q}^{0}+\varepsilon \widetilde{K}_{Q}(\varepsilon)\right)\left(\left(f^{\varepsilon}\right)^{*}\right)^{-1}+\varepsilon^{2} \widetilde{\mathcal{Y}}^{\prime}(\varepsilon),
\end{aligned}
$$

and

$$
\left\|\mathcal{Y}^{\prime}(\varepsilon)\right\|_{\mathfrak{G} \rightarrow \mathfrak{G}} \leq \mathcal{C}_{1}^{\prime}\left\|f^{-1}\right\|_{L_{\infty}}^{2}, \quad\left\|\widetilde{\mathcal{Y}}^{\prime}(\varepsilon)\right\|_{\mathfrak{G} \rightarrow \mathfrak{G}} \leq{\widetilde{\mathcal{C}_{1}^{\prime}}}^{\prime}\left\|f^{-1}\right\|_{L_{\infty}}^{2} .
$$

The structure of the corrector $K_{Q}(\varepsilon)$ in (9.5) is similar to (8.11). If $Q=I$, the correctors (9.5) and (9.6) coincide. In the general case, sometimes it is more convenient to use (9.5), and sometimes (9.6) is preferable.

9.2. The case where the corrector is equal to zero. This case is distinguished on the basis of Theorem 7.2. (It does not matter which corrector is considered.)

Theorem 9.2. Under the assumptions of Theorem 9.1, suppose that $\Lambda(\mathbf{x}) b(\boldsymbol{\theta})=0$ for all $\boldsymbol{\theta} \in \mathbb{S}^{d-1}, \mathrm{x} \in \Omega$. Then

$$
\begin{aligned}
\left\|\left(\widehat{\mathcal{A}}_{\varepsilon}+Q^{\varepsilon}\right)^{-1}-\left(\widehat{\mathcal{A}}^{0}+\bar{Q}\right)^{-1}\right\|_{\mathfrak{G} \rightarrow \mathfrak{G}} \leq \mathcal{C}_{2} \varepsilon^{2}, & \varepsilon>0, \\
\left\|\left(\mathcal{A}_{\varepsilon}+I\right)^{-1}-\left(f^{\varepsilon}\right)^{-1}\left(\widehat{\mathcal{A}}^{0}+\bar{Q}\right)^{-1}\left(\left(f^{\varepsilon}\right)^{*}\right)^{-1}\right\|_{\mathfrak{G} \rightarrow \mathfrak{G}} \leq \mathcal{C}_{3} \varepsilon^{2}, & \varepsilon>0 .
\end{aligned}
$$

Here $\mathcal{C}_{3}=\mathcal{C}_{2}\left\|f^{-1}\right\|_{L_{\infty}}^{2}$, and the constant $\mathcal{C}_{2}$ is defined by (5.25) and depends on $\|g\|_{L_{\infty}}$, $\left\|g^{-1}\right\|_{L_{\infty}},\|f\|_{L_{\infty}},\left\|f^{-1}\right\|_{L_{\infty}}, \alpha_{0}, \alpha_{1}$, and the parameters of the lattice $\Gamma$. In particular, (9.9) and (9.10) are true under conditions (3.9) (i.e., if $g^{0}=\bar{g}$ ).

9.3. "Elimination" of $\Pi_{\varepsilon}$. Now we find conditions under which $\Pi_{\varepsilon}$ may be replaced by $I$ in the first two terms of the corrector. The next statement follows from Theorem 7.5 by scaling. We also use Remark 7.6. 
Theorem 9.3. 1) Under the assumptions of Theorem 9.1(1), suppose that either Condition 6.6 or (the stronger) Condition 6.10 is satisfied. Then for $\varepsilon>0$ we have

$$
\begin{aligned}
\left(\widehat{\mathcal{A}}_{\varepsilon}+Q^{\varepsilon}\right)^{-1}= & \widehat{\mathcal{R}}_{Q}^{0}+\varepsilon K_{Q}^{\circ}(\varepsilon)+\varepsilon^{2} \check{\mathcal{I}}^{\prime \prime}(\varepsilon), \\
K_{Q}^{\circ}(\varepsilon)= & \Lambda_{Q}^{\varepsilon} b(\mathbf{D}) \widehat{\mathcal{R}}_{Q}^{0}+\widehat{\mathcal{R}}_{Q}^{0} b(\mathbf{D})^{*}\left(\Lambda_{Q}^{\varepsilon}\right)^{*}-\widehat{\mathcal{R}}_{Q}^{0} b(\mathbf{D})^{*} L_{Q}(\mathbf{D}) b(\mathbf{D}) \widehat{\mathcal{R}}_{Q}^{0}, \\
\left(\widehat{\mathcal{A}}_{\varepsilon}+Q^{\varepsilon}\right)^{-1}= & \widehat{\mathcal{R}}_{Q}^{0}+\varepsilon \widetilde{K}_{Q}^{\circ}(\varepsilon)+\varepsilon^{2} \widetilde{\mathcal{I}}^{\prime \prime}(\varepsilon), \\
\widetilde{K}_{Q}^{\circ}(\varepsilon)= & \Lambda^{\varepsilon} b(\mathbf{D}) \widehat{\mathcal{R}}_{Q}^{0}+\widehat{\mathcal{R}}_{Q}^{0} b(\mathbf{D})^{*}\left(\Lambda^{\varepsilon}\right)^{*} \\
& -\widehat{\mathcal{R}}_{Q}^{0}\left(b(\mathbf{D})^{*} L(\mathbf{D}) b(\mathbf{D})+b(\mathbf{D})^{*}(\overline{Q \Lambda})^{*}+(\overline{Q \Lambda}) b(\mathbf{D})\right) \widehat{\mathcal{R}}_{Q}^{0},
\end{aligned}
$$

and

$$
\left\|\check{\mathcal{I}}^{\prime \prime}(\varepsilon)\right\|_{\mathfrak{G} \rightarrow \mathfrak{G}} \leq \mathcal{C}_{1}^{\prime \prime}, \quad\left\|\tilde{\mathcal{I}}^{\prime \prime}(\varepsilon)\right\|_{\mathfrak{G} \rightarrow \mathfrak{G}} \leq \widetilde{\mathcal{C}}_{1}^{\prime \prime} .
$$

Here the constants $\mathcal{C}_{1}^{\prime \prime}$ and $\widetilde{\mathcal{C}}_{1}^{\prime \prime}$ are defined by (7.19) and (7.20) and depend on $\|g\|_{L_{\infty}}$, $\left\|g^{-1}\right\|_{L_{\infty}},\|Q\|_{L_{\infty}},\left\|Q^{-1}\right\|_{L_{\infty}}, \alpha_{0}, \alpha_{1}, m$, the parameters of the lattice, and also on the

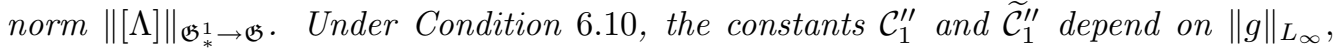
$\left\|g^{-1}\right\|_{L_{\infty}},\|Q\|_{L_{\infty}},\left\|Q^{-1}\right\|_{L_{\infty}}, \alpha_{0}, \alpha_{1}, m, n, d$, and the parameters of the lattice.

2) Under the conditions of Theorem 9.1(2) and Condition 6.6 (or Condition 6.10), for $\varepsilon>0$ we have

$$
\begin{aligned}
& \left(\mathcal{A}_{\varepsilon}+I\right)^{-1}=\left(f^{\varepsilon}\right)^{-1}\left(\widehat{\mathcal{R}}_{Q}^{0}+\varepsilon K_{Q}^{\circ}(\varepsilon)\right)\left(\left(f^{\varepsilon}\right)^{*}\right)^{-1}+\varepsilon^{2} \mathcal{Y}^{\prime \prime}(\varepsilon), \\
& \left(\mathcal{A}_{\varepsilon}+I\right)^{-1}=\left(f^{\varepsilon}\right)^{-1}\left(\widehat{\mathcal{R}}_{Q}^{0}+\varepsilon \widetilde{K}_{Q}^{\circ}(\varepsilon)\right)\left(\left(f^{\varepsilon}\right)^{*}\right)^{-1}+\varepsilon^{2} \widetilde{\mathcal{Y}}^{\prime \prime}(\varepsilon),
\end{aligned}
$$

and

$$
\left\|\mathcal{Y}^{\prime \prime}(\varepsilon)\right\|_{\mathfrak{G} \rightarrow \mathfrak{G}} \leq \mathcal{C}_{1}^{\prime \prime}\left\|f^{-1}\right\|_{L_{\infty}}^{2}, \quad\left\|\widetilde{\mathcal{Y}}^{\prime \prime}(\varepsilon)\right\|_{\mathfrak{G} \rightarrow \mathfrak{G}} \leq \widetilde{\mathcal{C}}_{1}^{\prime \prime}\left\|f^{-1}\right\|_{L_{\infty}}^{2} .
$$

Proposition 7.7 implies the following statement.

Proposition 9.4. Suppose that the conditions of Theorem 9.1 for the operator $\widehat{\mathcal{A}}_{\varepsilon}=$ $\mathbf{D}^{*} g^{\varepsilon} \mathbf{D}$ are satisfied, the matrix $g$ has real entries, and the function $Q$ is real-valued. Then for $\varepsilon>0$ we have

$$
\begin{aligned}
\left(\widehat{\mathcal{A}}_{\varepsilon}+Q^{\varepsilon}\right)^{-1} & =\widehat{\mathcal{R}}_{Q}^{0}+\varepsilon \Lambda^{\varepsilon} \mathbf{D} \widehat{\mathcal{R}}_{Q}^{0}+\varepsilon \widehat{\mathcal{R}}_{Q}^{0} \mathbf{D}^{*}\left(\Lambda^{\varepsilon}\right)^{*}+\varepsilon^{2} \check{\mathcal{I}}^{\times}(\varepsilon), \\
\left(\mathcal{A}_{\varepsilon}+I\right)^{-1} & =\left(f^{\varepsilon}\right)^{-1}\left(\widehat{\mathcal{R}}_{Q}^{0}+\varepsilon \Lambda^{\varepsilon} \mathbf{D} \widehat{\mathcal{R}}_{Q}^{0}+\varepsilon \widehat{\mathcal{R}}_{Q}^{0} \mathbf{D}^{*}\left(\Lambda^{\varepsilon}\right)^{*}\right)\left(\left(f^{\varepsilon}\right)^{*}\right)^{-1}+\varepsilon^{2} \mathcal{Y}^{\times}(\varepsilon),
\end{aligned}
$$

where

$$
\left\|\check{\mathcal{I}}^{\times}(\varepsilon)\right\|_{\mathfrak{G} \rightarrow \mathfrak{G}} \leq \mathcal{C}_{1}^{\times}, \quad\left\|\mathcal{Y}^{\times}(\varepsilon)\right\|_{\mathfrak{G} \rightarrow \mathfrak{G}} \leq \mathcal{C}_{1}^{\times}\left\|f^{-1}\right\|_{L_{\infty}}^{2} .
$$

Here the constant $\mathcal{C}_{1}^{\times}$is defined by (7.21) and depends on $\|g\|_{L_{\infty}},\left\|g^{-1}\right\|_{L_{\infty}},\|Q\|_{L_{\infty}}$, $\left\|Q^{-1}\right\|_{L_{\infty}}, d$, and the parameters of the lattice.

Finally, Proposition 7.8 yields the following.

Proposition 9.5. Under the assumptions of Theorem 9.1, suppose that conditions (3.10) are fulfilled (i.e., $g^{0}=\underline{g}$ ). Then in the representations (9.11) and (9.13) the corrector (9.12) simplifies and takes the form

$$
\widetilde{K}_{Q}^{\circ}(\varepsilon)=\Lambda^{\varepsilon} b(\mathbf{D}) \widehat{\mathcal{R}}_{Q}^{0}+\widehat{\mathcal{R}}_{Q}^{0} b(\mathbf{D})^{*}\left(\Lambda^{\varepsilon}\right)^{*}-\widehat{\mathcal{R}}_{Q}^{0}\left(b(\mathbf{D})^{*}(\overline{Q \Lambda})^{*}+(\overline{Q \Lambda}) b(\mathbf{D})\right) \widehat{\mathcal{R}}_{Q}^{0} .
$$

9.4. Approximation of the generalized resolvent for the family $\mathcal{A}_{\varepsilon}$. Now we consider the problem of approximation of the generalized resolvent for the family (9.1). Let $\mathfrak{Q}(\mathbf{x})$ be a $\Gamma$-periodic positive $(n \times n)$-matrix-valued function such that $\mathfrak{Q}, \mathfrak{Q}^{-1} \in L_{\infty}$. We factorize $\mathfrak{Q}$ :

$$
\mathfrak{Q}(\mathbf{x})=\left(\varphi(\mathbf{x}) \varphi(\mathbf{x})^{*}\right)^{-1},
$$


where the matrix-valued function $\varphi$ is $\Gamma$-periodic. We introduce the notation

$$
\psi(\mathbf{x}):=f(\mathbf{x}) \varphi(\mathbf{x}), \quad Q_{*}(\mathbf{x})=\left(\psi(\mathbf{x}) \psi(\mathbf{x})^{*}\right)^{-1}=\left(f(\mathbf{x})^{*}\right)^{-1} \mathfrak{Q}(\mathbf{x})(f(\mathbf{x}))^{-1} .
$$

By using the identity

$$
\left(\mathcal{A}_{\varepsilon}+\mathfrak{Q}^{\varepsilon}\right)^{-1}=\left(f^{\varepsilon}\right)^{-1}\left(\widehat{\mathcal{A}}_{\varepsilon}+Q_{*}^{\varepsilon}\right)^{-1}\left(\left(f^{\varepsilon}\right)^{*}\right)^{-1},
$$

the results for $\left(\mathcal{A}_{\varepsilon}+\mathfrak{Q}^{\varepsilon}\right)^{-1}$ can be deduced from the corresponding results on the approximation of the generalized resolvent $\left(\widehat{\mathcal{A}}_{\varepsilon}+Q_{*}^{\varepsilon}\right)^{-1}$. The next statement follows from Theorem 9.1.

Theorem 9.6. Let $\widehat{\mathcal{A}}_{\varepsilon}=\widehat{\mathcal{A}}\left(g^{\varepsilon}\right)$, and let $\widehat{\mathcal{A}}^{0}=\widehat{\mathcal{A}}\left(g^{0}\right)$ be the effective operator. Let $\mathcal{A}_{\varepsilon}=\left(f^{\varepsilon}\right)^{*} \widehat{\mathcal{A}}_{\varepsilon} f^{\varepsilon}$ be the operator (9.1). Suppose that $\mathfrak{Q}(\mathbf{x})$ is a periodic positive $(n \times n)$ matrix-valued function such that $\mathfrak{Q}, \mathfrak{Q}^{-1} \in L_{\infty}$ and that relations (9.15) and (9.16) are fulfilled. Let $\Lambda_{Q_{*}}(\mathbf{x})$ and $L_{Q_{*}}(\mathbf{D})$ be defined as in the assumptions of Theorem 9.1 with $Q$ replaced by $Q_{*}$. Let $\Pi_{\varepsilon}$ be the operator defined by (8.8). Then for $\varepsilon>0$ we have

$$
\begin{aligned}
& \left(\mathcal{A}_{\varepsilon}+\mathfrak{Q}^{\varepsilon}\right)^{-1} \\
& =\left(f^{\varepsilon}\right)^{-1}\left(\widehat{\mathcal{R}}_{Q_{*}}^{0}+\varepsilon \Lambda_{Q_{*}}^{\varepsilon} b(\mathbf{D}) \Pi_{\varepsilon} \widehat{\mathcal{R}}_{Q_{*}}^{0}+\varepsilon \widehat{\mathcal{R}}_{Q_{*}}^{0}\left(\Lambda_{Q_{*}}^{\varepsilon} b(\mathbf{D}) \Pi_{\varepsilon}\right)^{*}\right. \\
& \left.-\varepsilon \widehat{\mathcal{R}}_{Q_{*}}^{0} b(\mathbf{D})^{*} L_{Q_{*}}(\mathbf{D}) b(\mathbf{D}) \widehat{\mathcal{R}}_{Q_{*}}^{0}\right)\left(\left(f^{\varepsilon}\right)^{*}\right)^{-1}+\varepsilon^{2} \mathcal{Y}_{*}^{\prime}(\varepsilon), \\
& \left(\mathcal{A}_{\varepsilon}+\mathfrak{Q}^{\varepsilon}\right)^{-1} \\
& =\left(f^{\varepsilon}\right)^{-1}\left(\widehat{\mathcal{R}}_{Q_{*}}^{0}+\varepsilon \Lambda^{\varepsilon} b(\mathbf{D}) \Pi_{\varepsilon} \widehat{\mathcal{R}}_{Q_{*}}^{0}+\varepsilon \widehat{\mathcal{R}}_{Q_{*}}^{0}\left(\Lambda^{\varepsilon} b(\mathbf{D}) \Pi_{\varepsilon}\right)^{*}\right. \\
& \left.-\varepsilon \widehat{\mathcal{R}}_{Q_{*}}^{0}\left(b(\mathbf{D})^{*} L(\mathbf{D}) b(\mathbf{D})+b(\mathbf{D})^{*}\left(\overline{Q_{*} \Lambda}\right)^{*}+\left(\overline{Q_{*} \Lambda}\right) b(\mathbf{D})\right) \widehat{\mathcal{R}}_{Q_{*}}^{0}\right)\left(\left(f^{\varepsilon}\right)^{*}\right)^{-1} \\
& +\varepsilon^{2} \widetilde{\mathcal{Y}}_{*}^{\prime}(\varepsilon)
\end{aligned}
$$

where $\widehat{\mathcal{R}}_{Q_{*}}^{0}=\left(\widehat{\mathcal{A}}^{0}+\overline{Q_{*}}\right)^{-1}$, and

$$
\begin{aligned}
&\left\|\mathcal{Y}_{*}^{\prime}(\varepsilon)\right\|_{\mathfrak{G} \rightarrow \mathfrak{G}} \leq \mathcal{C}_{*}^{\prime}\left\|f^{-1}\right\|_{L_{\infty}}^{2}, \\
&\left\|\widetilde{\mathcal{Y}_{*}^{\prime}}(\varepsilon)\right\|_{\mathfrak{G} \rightarrow \mathfrak{G}} \leq \widetilde{\mathcal{C}}_{*}^{\prime}\left\|f^{-1}\right\|_{L_{\infty}}^{2} .
\end{aligned}
$$

Here the constant $\mathcal{C}_{*}^{\prime}$ is an analog of the constant $\mathcal{C}_{1}^{\prime}(c f .(7.16))$, while $\widetilde{\mathcal{C}}_{*}^{\prime}$ is an analog of $\widetilde{\mathcal{C}_{1}^{\prime}}(c f .(7.17))$, with $Q$ replaced by $Q_{*}$. The right-hand sides of (9.18) and (9.19) are expressed in terms of $\|g\|_{L_{\infty}},\left\|g^{-1}\right\|_{L_{\infty}},\|f\|_{L_{\infty}},\left\|f^{-1}\right\|_{L_{\infty}},\|\mathfrak{Q}\|_{L_{\infty}},\left\|\mathfrak{Q}^{-1}\right\|_{L_{\infty}}, \alpha_{0}, \alpha_{1}$, $m$, and the parameters of the lattice.

Similarly, Theorem 9.2 implies the following statement.

Theorem 9.7. Under the assumptions of Theorem 9.6, suppose that $\Lambda(\mathbf{x}) b(\boldsymbol{\theta})=0$ for all $\boldsymbol{\theta} \in \mathbb{S}^{d-1}, \mathrm{x} \in \Omega$. Then

$$
\left\|\left(\mathcal{A}_{\varepsilon}+\mathfrak{Q}^{\varepsilon}\right)^{-1}-\left(f^{\varepsilon}\right)^{-1}\left(\widehat{\mathcal{A}}^{0}+\overline{Q_{*}}\right)^{-1}\left(\left(f^{\varepsilon}\right)^{*}\right)^{-1}\right\|_{\mathfrak{G} \rightarrow \mathfrak{G}} \leq \mathcal{C}_{3}^{*} \varepsilon^{2}, \quad \varepsilon>0 .
$$

Here $\mathcal{C}_{3}^{*}=\mathcal{C}_{2}^{*}\left\|f^{-1}\right\|_{L_{\infty}}^{2}$, and $\mathcal{C}_{2}^{*}$ is an analog of the constant $\mathcal{C}_{2}$ (see (5.25)) with $Q$ replaced by $Q_{*}$. The constant $\mathcal{C}_{3}^{*}$ depends on $\|g\|_{L_{\infty}},\left\|g^{-1}\right\|_{L_{\infty}},\|f\|_{L_{\infty}},\left\|f^{-1}\right\|_{L_{\infty}},\|\mathfrak{Q}\|_{L_{\infty}}$, $\left\|\mathfrak{Q}^{-1}\right\|_{L_{\infty}}, \alpha_{0}, \alpha_{1}$, and the parameters of the lattice $\Gamma$. In particular, (9.20) is true under conditions (3.9) (i.e., if $g^{0}=\bar{g}$ ).

The next statement is deduced from Theorem 9.3. 
Theorem 9.8. Under the assumptions of Theorem 9.6, suppose that either Condition 6.6 or (the stronger) Condition 6.10 is satisfied. Then for $\varepsilon>0$ we have

$$
\begin{aligned}
& \left(\mathcal{A}_{\varepsilon}+\mathfrak{Q}^{\varepsilon}\right)^{-1} \\
& =\left(f^{\varepsilon}\right)^{-1}\left(\widehat{\mathcal{R}}_{Q_{*}}^{0}+\varepsilon \Lambda_{Q_{*}}^{\varepsilon} b(\mathbf{D}) \widehat{\mathcal{R}}_{Q_{*}}^{0}+\varepsilon \widehat{\mathcal{R}}_{Q_{*}}^{0} b(\mathbf{D})^{*}\left(\Lambda_{Q_{*}}^{\varepsilon}\right)^{*}\right. \\
& \left.-\varepsilon \widehat{\mathcal{R}}_{Q_{*}}^{0} b(\mathbf{D})^{*} L_{Q_{*}}(\mathbf{D}) b(\mathbf{D}) \widehat{\mathcal{R}}_{Q_{*}}^{0}\right)\left(\left(f^{\varepsilon}\right)^{*}\right)^{-1}+\varepsilon^{2} \mathcal{Y}_{*}^{\prime \prime}(\varepsilon), \\
& \left(\mathcal{A}_{\varepsilon}+\mathfrak{Q}^{\varepsilon}\right)^{-1} \\
& =\left(f^{\varepsilon}\right)^{-1}\left(\widehat{\mathcal{R}}_{Q_{*}}^{0}+\varepsilon \Lambda^{\varepsilon} b(\mathbf{D}) \widehat{\mathcal{R}}_{Q_{*}}^{0}+\varepsilon \widehat{\mathcal{R}}_{Q_{*}}^{0} b(\mathbf{D})^{*}\left(\Lambda^{\varepsilon}\right)^{*}\right. \\
& \left.-\varepsilon \widehat{\mathcal{R}}_{Q_{*}}^{0}\left(b(\mathbf{D})^{*} L(\mathbf{D}) b(\mathbf{D})+b(\mathbf{D})^{*}\left(\overline{Q_{*} \Lambda}\right)^{*}+\left(\overline{Q_{*} \Lambda}\right) b(\mathbf{D})\right) \widehat{\mathcal{R}}_{Q_{*}}^{0}\right)\left(\left(f^{\varepsilon}\right)^{*}\right)^{-1} \\
& +\varepsilon^{2} \widetilde{\mathcal{Y}}_{*}^{\prime \prime}(\varepsilon) \\
& \left\|\mathcal{Y}_{*}^{\prime \prime}(\varepsilon)\right\|_{\mathfrak{G} \rightarrow \mathfrak{G}} \leq \mathcal{C}_{*}^{\prime \prime}\left\|f^{-1}\right\|_{L_{\infty}}^{2}, \\
& \left\|\widetilde{\mathcal{Y}}_{*}^{\prime \prime}(\varepsilon)\right\|_{\mathfrak{G} \rightarrow \mathfrak{G}} \leq{\widetilde{\mathcal{C}_{*}^{\prime \prime}}}^{\prime \prime}\left\|f^{-1}\right\|_{L_{\infty}}^{2} \text {. }
\end{aligned}
$$

Here the constant $\mathcal{C}_{*}^{\prime \prime}$ is an analog of the constant $\mathcal{C}_{1}^{\prime \prime}$ (see $\left.(7.19)\right)$, and $\widetilde{\mathcal{C}}_{*}^{\prime \prime}$ is an analog of the constant $\widetilde{\mathcal{C}_{1}^{\prime \prime}}($ see $(7.20))$, with $Q$ replaced by $Q_{*}$. The right-hand sides of $(9.21)$ and (9.22) are expressed in terms of $\|g\|_{L_{\infty}},\left\|g^{-1}\right\|_{L_{\infty}},\|f\|_{L_{\infty}},\left\|f^{-1}\right\|_{L_{\infty}},\|\mathfrak{Q}\|_{L_{\infty}},\left\|\mathfrak{Q}^{-1}\right\|_{L_{\infty}}$,

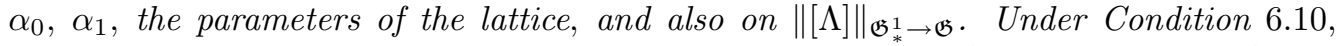
the right-hand sides of (9.21) and (9.22) depend on $\|g\|_{L_{\infty}},\left\|g^{-1}\right\|_{L_{\infty}},\|f\|_{L_{\infty}},\left\|f^{-1}\right\|_{L_{\infty}}$, $\|\mathfrak{Q}\|_{L_{\infty}},\left\|\mathfrak{Q}^{-1}\right\|_{L_{\infty}}, \alpha_{0}, \alpha_{1}, m, n, d$, and the parameters of the lattice.

Proposition 9.4 yields the following statement.

Proposition 9.9. Suppose that the operator $\widehat{\mathcal{A}}_{\varepsilon}=\mathbf{D}^{*} g^{\varepsilon} \mathbf{D}$ satisfies the assumptions of Theorem 9.6, the matrix $g(\mathbf{x})$ has real entries, and the function $\mathfrak{Q}(\mathbf{x})$ is real-valued. Then

$$
\left(\mathcal{A}_{\varepsilon}+\mathfrak{Q}^{\varepsilon}\right)^{-1}=\left(f^{\varepsilon}\right)^{-1}\left(\widehat{\mathcal{R}}_{Q_{*}}^{0}+\varepsilon \Lambda^{\varepsilon} \mathbf{D} \widehat{\mathcal{R}}_{Q_{*}}^{0}+\varepsilon \widehat{\mathcal{R}}_{Q_{*}}^{0} \mathbf{D}^{*}\left(\Lambda^{\varepsilon}\right)^{*}\right)\left(\left(f^{\varepsilon}\right)^{*}\right)^{-1}+\varepsilon^{2} \mathcal{I}_{*}^{\times}(\varepsilon)
$$

and

$$
\left\|\mathcal{I}_{*}^{\times}(\varepsilon)\right\|_{\mathfrak{G} \rightarrow \mathfrak{G}} \leq \mathcal{C}_{*}^{\times}\left\|f^{-1}\right\|_{L_{\infty}}^{2}=: \mathcal{C}^{\times} .
$$

Here the constant $\mathcal{C}_{*}^{\times}$is an analog of the constant $\mathcal{C}_{1}^{\times}$(see (7.21)) with $Q$ replaced by $Q_{*}$. The constant $\mathcal{C}^{\times}$depends on $\|g\|_{L_{\infty}},\left\|g^{-1}\right\|_{L_{\infty}},\|f\|_{L_{\infty}},\left\|f^{-1}\right\|_{L_{\infty}},\|\mathfrak{Q}\|_{L_{\infty}},\left\|\mathfrak{Q}^{-1}\right\|_{L_{\infty}}, d$, and the parameters of the lattice.

Finally, combining Proposition 9.5 with (9.17), we obtain the following.

Proposition 9.10. Under the assumptions of Theorem 9.6, suppose that conditions (3.10) are fulfilled (i.e., $g^{0}=\underline{g}$ ). Then for $\varepsilon>0$ we have

$$
\begin{aligned}
\left(\mathcal{A}_{\varepsilon}+\mathfrak{Q}^{\varepsilon}\right)^{-1}= & \left(f^{\varepsilon}\right)^{-1}\left(\widehat{\mathcal{R}}_{Q_{*}}^{0}+\varepsilon \Lambda^{\varepsilon} b(\mathbf{D}) \widehat{\mathcal{R}}_{Q_{*}}^{0}+\varepsilon \widehat{\mathcal{R}}_{Q_{*}}^{0} b(\mathbf{D})^{*}\left(\Lambda^{\varepsilon}\right)^{*}\right. \\
& \left.\quad-\varepsilon \widehat{\mathcal{R}}_{Q_{*}}^{0}\left(b(\mathbf{D})^{*}\left(\overline{Q_{*} \Lambda}\right)^{*}+\left(\overline{Q_{*} \Lambda}\right) b(\mathbf{D})\right) \widehat{\mathcal{R}}_{Q_{*}}^{0}\right)\left(\left(f^{\varepsilon}\right)^{*}\right)^{-1} \\
& +\varepsilon^{2} \widetilde{\mathcal{Y}}_{*}^{\prime \prime}(\varepsilon)
\end{aligned}
$$

where $\widetilde{\mathcal{Y}}_{*}^{\prime \prime}(\varepsilon)$ satisfies $(9.22)$. Now the constant $\widetilde{\mathcal{C}}_{*}^{\prime \prime}$ depends only on $\|g\|_{L_{\infty}},\left\|g^{-1}\right\|_{L_{\infty}}$, $\|f\|_{L_{\infty}},\left\|f^{-1}\right\|_{L_{\infty}},\|\mathfrak{Q}\|_{L_{\infty}},\left\|\mathfrak{Q}^{-1}\right\|_{L_{\infty}}, m, n, d$, and the parameters of the lattice. 
9.5. The weak limit of the corrector. In conclusion, we briefly dwell on the analogs of the statements of Subsection 8.6, as applied to the generalized resolvent $\left(\widehat{\mathcal{A}}_{\varepsilon}+Q^{\varepsilon}\right)^{-1}$. Here, it is more convenient to use the correctors $\widetilde{K}_{Q}(\varepsilon)$ and $\widetilde{K}_{Q}^{\circ}(\varepsilon)$ (see (9.6) and (9.12)). The following statement is easy to check.

Proposition 9.11. 1) Under the assumptions of Theorem 9.1(1), we have

$$
\mathrm{w}_{\varepsilon \rightarrow 0} \lim _{Q}(\varepsilon)=\widetilde{K}_{Q, 3},
$$

where $\widetilde{K}_{Q, 3}$ is the last (the third) summand of the corrector $\widetilde{K}_{Q}(\varepsilon)$ in (9.6). Moreover, the operator-valued function $\left(\widehat{\mathcal{A}}_{\varepsilon}+Q^{\varepsilon}\right)^{-1}$ admits a weak derivative with respect to $\varepsilon$ at the point $\varepsilon=0$. This derivative is equal to the operator $\widetilde{K}_{Q, 3}$.

2) Under the assumptions of Theorem 9.3(1), we have

$$
\mathrm{w}_{\varepsilon \rightarrow 0} \lim _{Q}^{\circ}(\varepsilon)=\widetilde{K}_{Q, 3} .
$$

Similar statements are true for the resolvents $\left(\mathcal{A}_{\varepsilon}+I\right)^{-1}$ and $\left(\mathcal{A}_{\varepsilon}+\mathfrak{Q}^{\varepsilon}\right)^{-1}$, but the formulations are bulkier, and we shall not dwell on this.

\section{Chapter 4. Applications of the general theory}

We proceed to applications of the general results to particular periodic operators of mathematical physics. Almost all examples that we consider were studied in [BSu2], but without taking the corrector into account. An exception is the periodic magnetic Schrödinger operator (see Subsection 11.3), which has not been discussed before. Taken together, the examples considered below verify the realizability of all cases occurring in the general theorems of Chapter 3. Formally, the exposition in the present chapter does not depend on the paper BSu2]. However, acquaintance with Chapters 5 and 6 of [BSu2] may be useful.

\section{§10. The operator $\widehat{\mathcal{A}}=\mathbf{D}^{*} g \mathbf{D}$}

10.1. The case where the matrix $g(x)$ has real entries. We start with the operator

$$
\widehat{\mathcal{A}}=\mathbf{D}^{*} g(\mathbf{x}) \mathbf{D}=-\operatorname{div} g(\mathbf{x}) \nabla
$$

acting in $\mathfrak{G}=L_{2}\left(\mathbb{R}^{d}\right), d \geq 1(\mathrm{cf}$. BSu2, $\left.\S 5.1]\right)$. Here $g(\mathbf{x})$ is a $\Gamma$-periodic $(d \times d)$-matrixvalued function with real entries and such that

$$
g(\mathbf{x})>0, \quad g, g^{-1} \in L_{\infty} .
$$

The operator (10.1) describes a periodic acoustic medium; it also occurs in diffusion problems, etc. For us, this operator is important also as a basic object in the study of the periodic Schrödinger operator. Now $n=1, m=d, b(\xi)=\xi$. Obviously, conditions (2.1) and (2.2) are satisfied with $\alpha_{0}=\alpha_{1}=1$. The solutions $v_{j} \in \widetilde{H}^{1}(\Omega)$ of the equations of the form (3.4) with $\mathbf{C}=\mathbf{e}_{j}, j=1, \ldots, d$, are pure imaginary. Therefore, it is convenient to introduce the solutions $\Phi_{j} \in \widetilde{H}^{1}(\Omega), j=1, \ldots, d$, of the equations

$$
\operatorname{div} g(\mathbf{x})\left(\nabla \Phi_{j}(\mathbf{x})+\mathbf{e}_{j}\right)=0, \quad \int_{\Omega} \Phi_{j}(\mathbf{x}) d \mathbf{x}=0 .
$$

Then the functions $\Phi_{j}(\mathbf{x})$ are real-valued, $v_{j}(\mathbf{x})=i \Phi_{j}(\mathbf{x}), \Lambda(\mathbf{x})$ is a row-matrix:

$$
\Lambda(\mathbf{x})=i\left(\Phi_{1}(\mathbf{x}), \ldots, \Phi_{d}(\mathbf{x})\right),
$$

$\widetilde{g}(\mathbf{x})$ is a real $(d \times d)$-matrix with the columns $g(\mathbf{x})\left(\nabla \Phi_{j}(\mathbf{x})+\mathbf{e}_{j}\right), j=1, \ldots, d$, and $g^{0}=|\Omega|^{-1} \int_{\Omega} \widetilde{g}(\mathbf{x}) d \mathbf{x}$. Finally, $\Lambda b(\mathbf{D})=\Lambda \mathbf{D}=\sum_{j=1}^{d} \Phi_{j}(\mathbf{x}) \partial_{j}$. 
We consider the operator $\widehat{\mathcal{A}}_{\varepsilon}=\mathbf{D}^{*} g^{\varepsilon}(\mathbf{x}) \mathbf{D}$ with a rapidly oscillating matrix $g^{\varepsilon}(\mathbf{x})$. Proposition 8.4 applies, which yields the following statement.

Theorem 10.1. Let $\widehat{\mathcal{A}}(g)=\mathbf{D}^{*} g(\mathbf{x}) \mathbf{D}$, where a $\Gamma$-periodic matrix $g(\mathbf{x})$ satisfies $(10.2)$. Let $\widehat{\mathcal{A}}^{0}=\widehat{\mathcal{A}}\left(g^{0}\right)$ be the effective operator, let $\widehat{\mathcal{A}}_{\varepsilon}=\widehat{\mathcal{A}}\left(g^{\varepsilon}\right)$, and let $\Phi_{j} \in \widetilde{H}^{1}(\Omega)$ be the solution of problem (10.3), $j=1, \ldots, d$. Then for $\varepsilon>0$ we have

$$
\left(\widehat{\mathcal{A}}_{\varepsilon}+I\right)^{-1}=\widehat{\mathcal{R}}^{0}+\varepsilon \sum_{j=1}^{d} \Phi_{j}^{\varepsilon} \partial_{j} \widehat{\mathcal{R}}^{0}-\varepsilon \widehat{\mathcal{R}}^{0} \sum_{j=1}^{d} \partial_{j} \Phi_{j}^{\varepsilon}+\varepsilon^{2} \widehat{\mathcal{I}}^{\times}(\varepsilon),
$$

where $\widehat{\mathcal{R}}^{0}=\left(\widehat{\mathcal{A}}^{0}+I\right)^{-1}$ and

$$
\left\|\widehat{\mathcal{I}}^{\times}(\varepsilon)\right\|_{L_{2}\left(\mathbb{R}^{d}\right) \rightarrow L_{2}\left(\mathbb{R}^{d}\right)} \leq \widehat{\mathcal{C}}_{1}^{\times} .
$$

The constant $\widehat{\mathcal{C}_{1}^{\times}}$depends only on $\|g\|_{L_{\infty}},\left\|g^{-1}\right\|_{L_{\infty}}, d$, and the parameters of the lattice $\Gamma$.

In fact, the problem of approximation of the generalized resolvent $\left(\widehat{\mathcal{A}}_{\varepsilon}+Q^{\varepsilon}\right)^{-1}$ was solved in Proposition 9.4. Now we rewrite (9.14) in a "decoded" form.

Theorem 10.2. Under the assumptions of Theorem 10.1, let $Q(\mathbf{x})$ be a real-valued periodic function such that $Q(\mathbf{x})>0$ and $Q, Q^{-1} \in L_{\infty}$. Then for $\varepsilon>0$ we have

$$
\left(\widehat{\mathcal{A}}_{\varepsilon}+Q^{\varepsilon}\right)^{-1}=\widehat{\mathcal{R}}_{Q}^{0}+\varepsilon \sum_{j=1}^{d} \Phi_{j}^{\varepsilon} \partial_{j} \widehat{\mathcal{R}}_{Q}^{0}-\varepsilon \widehat{\mathcal{R}}_{Q}^{0} \sum_{j=1}^{d} \partial_{j} \Phi_{j}^{\varepsilon}+\varepsilon^{2} \check{\mathcal{I}}^{\times}(\varepsilon),
$$

where $\widehat{\mathcal{R}}_{Q}^{0}=\left(\widehat{\mathcal{A}}^{0}+\bar{Q}\right)^{-1}$, and

$$
\left\|\check{\mathcal{I}}^{\times}(\varepsilon)\right\|_{L_{2}\left(\mathbb{R}^{d}\right) \rightarrow L_{2}\left(\mathbb{R}^{d}\right)} \leq \mathcal{C}_{1}^{\times} .
$$

The constant $\mathcal{C}_{1}^{\times}$depends only on $\|g\|_{L_{\infty}},\left\|g^{-1}\right\|_{L_{\infty}},\|Q\|_{L_{\infty}},\left\|Q^{-1}\right\|_{L_{\infty}}$, d, and the parameters of the lattice $\Gamma$.

10.2. Interpolation. In the recent paper [Zh4, Zhikov obtained an estimate that can be written in our terms as follows.

Theorem 10.3 [h4]. Under the assumptions of Theorem 10.1, the operator (10.1) satisfies the estimate

$$
\left\|\left(\widehat{\mathcal{A}}_{\varepsilon}+I\right)^{-1}-\widehat{\mathcal{R}}^{0}-\varepsilon K_{1}^{\circ}(\varepsilon)\right\|_{L_{2}\left(\mathbb{R}^{d}\right) \rightarrow H^{1}\left(\mathbb{R}^{d}\right)} \leq c \varepsilon .
$$

Here

$$
K_{1}^{\circ}(\varepsilon)=\sum_{j=1}^{d} \Phi_{j}^{\varepsilon} \partial_{j} \widehat{\mathcal{R}}^{0},
$$

and the constant $c$ depends only on $\|g\|_{L_{\infty}},\left\|g^{-1}\right\|_{L_{\infty}}, d$, and the parameters of the lattice.

In our terms, the operator $K_{1}^{\circ}(\varepsilon)$ is the "truncated" corrector. However (see Subsection 8.5), estimate (10.8) remains valid if the truncated corrector is replaced by the full corrector $K^{\circ}(\varepsilon)$, which now has the form

$$
K^{\circ}(\varepsilon)=K_{1}^{\circ}(\varepsilon)+\left(K_{1}^{\circ}(\varepsilon)\right)^{*} .
$$

Herewith, the constant $c$ will change. Estimate (10.8) with $K_{1}^{\circ}(\varepsilon)$ replaced by the corrector (10.10) can be combined with the representation (10.4), (10.5); this leads to the relation

$$
\left\|\left(\widehat{\mathcal{A}}_{\varepsilon}+I\right)^{-1}-\widehat{\mathcal{R}}^{0}-\varepsilon K^{\circ}(\varepsilon)\right\|_{L_{2}\left(\mathbb{R}^{d}\right) \rightarrow H^{j}\left(\mathbb{R}^{d}\right)} \leq c_{j} \varepsilon^{2-j}, \quad j=0,1 .
$$

Here $H^{0}\left(\mathbb{R}^{d}\right):=L_{2}\left(\mathbb{R}^{d}\right)$.

Now, the simplest interpolation yields the following result. 
Proposition 10.4. Under the assumptions of Theorem 10.1, let $K^{\circ}(\varepsilon)$ be the operator defined by (10.9) and (10.10). Then

$$
\left\|\left(\widehat{\mathcal{A}}_{\varepsilon}+I\right)^{-1}-\widehat{\mathcal{R}}^{0}-\varepsilon K^{\circ}(\varepsilon)\right\|_{L_{2}\left(\mathbb{R}^{d}\right) \rightarrow H^{s}\left(\mathbb{R}^{d}\right)} \leq c_{0}^{1-s} c_{1}^{s} \varepsilon^{2-s}, \quad 0<s<1 .
$$

The constants $c_{0}$ and $c_{1}$ depend only on $\|g\|_{L_{\infty}},\left\|g^{-1}\right\|_{L_{\infty}}, d$, and the parameters of the lattice.

We note that, in general, for any $s$ in (10.11), it is impossible to replace the corrector (10.10) by the truncated corrector (10.9). At the same time, we have seen that for $s=1$ this is possible.

However, there is a particular case, namely, the case where $g^{0}=\bar{g}$, when interpolation simplifies. (Now we need a forward reference because particular cases will be considered later for matrices $g$ with complex entries.) The condition $g^{0}=\bar{g}$ is equivalent to (10.17), and estimate (10.18) is true. If $g^{0}=\bar{g}$, the corrector (10.9) in (10.8) is equal to zero. If $g^{0}=\bar{g}$, then, by interpolation, instead of (10.11) we obtain the estimate

$$
\left\|\left(\widehat{\mathcal{A}}_{\varepsilon}+I\right)^{-1}-\widehat{\mathcal{R}}^{0}\right\|_{L_{2}\left(\mathbb{R}^{d}\right) \rightarrow H^{s}\left(\mathbb{R}^{d}\right)} \leq c_{0}^{1-s} c_{1}^{s} \varepsilon^{2-s}, \quad 0<s<1 .
$$

10.3. The case where the matrix $g(x)$ has complex entries. Now we consider the operator (10.1), assuming that $g(\mathbf{x})$ is a periodic Hermitian matrix with complex entries and satisfying (10.2). In general, in this case the third term of the corrector is nonzero. Now the solutions $\Phi_{j}(\mathbf{x})$ of problem (10.3) are complex-valued. We calculate $L(\mathbf{k})$. By (4.8), $L(\mathbf{k})$ is the $(d \times d)$-matrix with the entries

$$
L_{j l}(\mathbf{k})=i \sum_{s=1}^{d}\left(a_{l j s}^{*}-a_{j l s}\right) k_{s}, \quad j, l=1, \ldots, d,
$$

where

$$
a_{j l s}:=|\Omega|^{-1} \int_{\Omega} \Phi_{j}(\mathbf{x})^{*}\left\langle g(\mathbf{x})\left(\nabla \Phi_{l}(\mathbf{x})+\mathbf{e}_{l}\right), \mathbf{e}_{s}\right\rangle d \mathbf{x}, \quad j, l, s=1, \ldots, d .
$$

Then

$$
\mathbf{D}^{*} L(\mathbf{D}) \mathbf{D}=\sum_{j, l, s=1}^{d}\left(a_{j l s}-a_{j l s}^{*}\right) \partial_{j} \partial_{l} \partial_{s} .
$$

Theorem 8.1 applies. Unlike the case where the matrix $g(\mathbf{x})$ has real entries, now we cannot use the boundedness of the solutions $\Phi_{j}(\mathbf{x})$. Therefore, in the general case we cannot replace $\Pi_{\varepsilon}$ by $I$ in (8.9). Of course, such a replacement is possible if $d \leq 4$ (as well as for general matrix operators), or if $g^{0}=\underline{g}$. Also, the solutions $\Phi_{j}(\mathbf{x})$ are bounded under some additional conditions on $\operatorname{Im} g(\mathbf{x})$. Indeed, write the matrix $g(\mathbf{x})$ as $g(\mathbf{x})=g_{1}(\mathbf{x})+i g_{2}(\mathbf{x})$, where $g_{1}(\mathbf{x})$ is a symmetric matrix with real entries, and $g_{2}(\mathbf{x})$ is an antisymmetric matrix with real entries. The solutions $\Phi_{j}(\mathbf{x})$ of problem (10.3) can be represented as $\Phi_{j}(\mathbf{x})=\Phi_{j}^{(1)}(\mathbf{x})+i \Phi_{j}^{(2)}(\mathbf{x})$, where $\Phi_{j}^{(1)}(\mathbf{x})$ and $\Phi_{j}^{(2)}(\mathbf{x})$ are real-valued functions. Then problem (10.3) can be rewritten as the following system of equations with real coefficients:

$$
\left\{\begin{array}{l}
\operatorname{div} g_{1}(\mathbf{x}) \nabla \Phi_{j}^{(1)}(\mathbf{x})-\sum_{l=1}^{d} \mathfrak{A}_{l}(\mathbf{x}) \partial_{l} \Phi_{j}^{(2)}(\mathbf{x})=-\operatorname{div} g_{1}(\mathbf{x}) \mathbf{e}_{j}, \\
\sum_{l=1}^{d} \mathfrak{A}_{l}(\mathbf{x}) \partial_{l} \Phi_{j}^{(1)}(\mathbf{x})+\operatorname{div} g_{1}(\mathbf{x}) \nabla \Phi_{j}^{(2)}(\mathbf{x})=-\operatorname{div} g_{2}(\mathbf{x}) \mathbf{e}_{j}
\end{array}\right.
$$


under the conditions $\int_{\Omega} \Phi_{j}^{(1)}(\mathbf{x}) d \mathbf{x}=\int_{\Omega} \Phi_{j}^{(2)}(\mathbf{x}) d \mathbf{x}=0$. Here

$$
\mathfrak{A}_{j}(\mathbf{x})=\sum_{l=1}^{d} \partial_{l} g_{2}^{(l j)}(\mathbf{x})=\operatorname{div} \mathbf{g}_{2}^{(j)}(\mathbf{x}), \quad j=1, \ldots, d,
$$

and the $\mathbf{g}_{2}^{(j)}(\mathbf{x})$ are the columns of the matrix $g_{2}(\mathbf{x})$. Suppose that, for some $q>d$,

$$
\operatorname{div} \mathbf{g}_{2}^{(j)} \in L_{q}(\Omega), \quad q>d, j=1, \ldots, d .
$$

System (10.13) is a system "with diagonal leading part" and satisfies the assumptions of Theorem 2.1 in $\left[\mathrm{LaU}\right.$, Chapter VII, §2]. By this theorem, we have $\Phi_{j} \in L_{\infty}$, and the norm $\left\|\Phi_{j}\right\|_{L_{\infty}}$ is estimated by a constant depending on $\left\|g_{1}\right\|_{L_{\infty}},\left\|g_{1}^{-1}\right\|_{L_{\infty}}, d, \Omega$, and the norms $\left\|\mathfrak{A}_{l}\right\|_{L_{q}(\Omega)}, l=1, \ldots, d$. Thus, under conditions $(10.14), \Lambda \in L_{\infty}$. Therefore, Condition 6.6 is satisfied. Then Theorem 8.2 applies.

Combining Theorems 8.1 and 8.2 and applying them to the operator (10.1), we arrive at the following statement.

Theorem 10.5. 1) Let $\widehat{\mathcal{A}}(g)=\mathbf{D}^{*} g(\mathbf{x}) \mathbf{D}$, where $g(\mathbf{x})$ is a $\Gamma$-periodic matrix with complex entries and satisfying (10.2), let $\widehat{\mathcal{A}}^{0}=\widehat{\mathcal{A}}\left(g^{0}\right)$ be the effective operator, and let $\widehat{\mathcal{A}}_{\varepsilon}=$ $\widehat{\mathcal{A}}\left(g^{\varepsilon}\right)$. Let $\Phi_{j} \in \widetilde{H}^{1}(\Omega)$ be the solution of problem $(10.3), j=1, \ldots, d$, and let $a_{j l s}$, $j, l, s=1, \ldots, d$, be the numbers defined by (10.12). Let $\Pi_{\varepsilon}$ be the operator defined by (8.8). Then for $\varepsilon>0$ we have

$$
\begin{aligned}
\left(\widehat{\mathcal{A}}_{\varepsilon}+I\right)^{-1}= & \widehat{\mathcal{R}}^{0}+\varepsilon \sum_{j=1}^{d} \Phi_{j}^{\varepsilon} \partial_{j} \Pi_{\varepsilon} \widehat{\mathcal{R}}^{0}-\varepsilon \widehat{\mathcal{R}}^{0} \Pi_{\varepsilon} \sum_{j=1}^{d} \partial_{j}\left(\Phi_{j}^{\varepsilon}\right)^{*} \\
& -\varepsilon \sum_{j, l, s=1}^{d} \widehat{\mathcal{R}}^{0}\left(a_{j l s}-a_{j l s}^{*}\right) \partial_{j} \partial_{l} \partial_{s} \widehat{\mathcal{R}}^{0}+\varepsilon^{2} \widehat{\mathcal{I}}^{\prime}(\varepsilon),
\end{aligned}
$$

where $\widehat{\mathcal{R}}^{0}=\left(\widehat{\mathcal{A}}^{0}+I\right)^{-1}$, and

$$
\left\|\widehat{\mathcal{I}}^{\prime}(\varepsilon)\right\|_{L_{2}\left(\mathbb{R}^{d}\right) \rightarrow L_{2}\left(\mathbb{R}^{d}\right)} \leq \widehat{\mathcal{C}}_{1}^{\prime}
$$

The constant $\widehat{\mathcal{C}}_{1}^{\prime}$ depends only on $\|g\|_{L_{\infty}},\left\|g^{-1}\right\|_{L_{\infty}}$, and the parameters of the lattice $\Gamma$.

2) Under the same assumptions as in 1), suppose that the operators of multiplication by $\Phi_{j}(\mathbf{x}), j=1, \ldots, d$, are continuous operators from $H^{1}\left(\mathbb{R}^{d}\right)$ to $L_{2}\left(\mathbb{R}^{d}\right)$. (This condition is fulfilled a fortiori if $d \leq 4$, or if $g^{0}=\underline{g}$, or if the columns $\mathbf{g}_{2}^{(j)}(\mathbf{x}), j=1, \ldots, d$, of the matrix $g_{2}(\mathbf{x})=\operatorname{Im} g(\mathbf{x})$ satisfy (10.14).) Then

$$
\begin{aligned}
\left(\widehat{\mathcal{A}}_{\varepsilon}+I\right)^{-1}= & \widehat{\mathcal{R}}^{0}+\varepsilon \sum_{j=1}^{d} \Phi_{j}^{\varepsilon} \partial_{j} \widehat{\mathcal{R}}^{0}-\varepsilon \widehat{\mathcal{R}}^{0} \sum_{j=1}^{d} \partial_{j}\left(\Phi_{j}^{\varepsilon}\right)^{*} \\
& -\varepsilon \sum_{j, l, s=1}^{d} \widehat{\mathcal{R}}^{0}\left(a_{j l s}-a_{j l s}^{*}\right) \partial_{j} \partial_{l} \partial_{s} \widehat{\mathcal{R}}^{0}+\varepsilon^{2} \widehat{\mathcal{I}}^{\prime \prime}(\varepsilon)
\end{aligned}
$$

and

$$
\left\|\widehat{\mathcal{I}}^{\prime \prime}(\varepsilon)\right\|_{L_{2}\left(\mathbb{R}^{d}\right) \rightarrow L_{2}\left(\mathbb{R}^{d}\right)} \leq \widehat{\mathcal{C}}_{1}^{\prime \prime},
$$

where $\widehat{\mathcal{C}}_{1}^{\prime \prime}$ depends only on $\|g\|_{L_{\infty}},\left\|g^{-1}\right\|_{L_{\infty}}, d$, the parameters of the lattice $\Gamma$, and also on the norms $\left\|\left[\Phi_{j}\right]\right\|_{H^{1}\left(\mathbb{R}^{d}\right) \rightarrow L_{2}\left(\mathbb{R}^{d}\right)}, j=1, \ldots, d$.

It should be noted that for $d \leq 4$, or for $g^{0}=\underline{g}$, the norms $\left\|\left[\Phi_{j}\right]\right\|_{H^{1}\left(\mathbb{R}^{d}\right) \rightarrow L_{2}\left(\mathbb{R}^{d}\right)}$ themselves are estimated by $\|g\|_{L_{\infty}},\left\|g^{-1}\right\|_{L_{\infty}}, d$, and the parameters of the lattice. 
10.4. Example. It is easy to give an example of a Hermitian matrix $g(\mathbf{x})$ with complex entries such that the third term of the corrector is nonzero. Let $d=2$, let $\Gamma=(2 \pi \mathbb{Z})^{2}$, and let

$$
g(\mathbf{x})=\left(\begin{array}{cc}
1 & i \beta^{\prime}\left(x_{1}\right) \\
-i \beta^{\prime}\left(x_{1}\right) & 1
\end{array}\right)
$$

where $\beta\left(x_{1}\right)$ is a smooth $(2 \pi)$-periodic real-valued function such that $\int_{0}^{2 \pi} \beta\left(x_{1}\right) d x_{1}=0$ and $1-\left(\beta^{\prime}\left(x_{1}\right)\right)^{2}>0$. Here $\beta^{\prime}\left(x_{1}\right)$ is the derivative of the function $\beta\left(x_{1}\right)$. Calculations show that $\mathbf{D}^{*} L(\mathbf{D}) \mathbf{D}=-\pi^{-1} \alpha D_{2}^{3}$, where $\alpha=\int_{0}^{2 \pi} \beta\left(x_{1}\right)\left(\beta^{\prime}\left(x_{1}\right)\right)^{2} d x_{1}$. For instance, the function $\beta\left(x_{1}\right)=c\left(\sin x_{1}+\cos 2 x_{1}\right)$ with $0<c<1 / 3$ satisfies the above assumptions, and $\alpha=-(3 \pi / 2) c^{3} \neq 0$. The presence of the nontrivial third term in the corrector corresponds to the fact that the coefficient $\widehat{\mu}(\boldsymbol{\theta})$ in the decomposition (see Subsection 3.3) of the analytic eigenvalue $\widehat{\lambda}(t, \boldsymbol{\theta})=\widehat{\gamma}(\boldsymbol{\theta}) t^{2}+\widehat{\mu}(\boldsymbol{\theta}) t^{3}+\cdots$ is not equal to zero. In our example, $\widehat{\mu}(\boldsymbol{\theta})=-\pi^{-1} \alpha \theta_{2}^{3} \neq 0$ for $\theta_{2} \neq 0$.

10.5. Theorems 9.1 and 9.3 can also be realized in the complex case. We formulate the results simultaneously for the generalized resolvent $\left(\widehat{\mathcal{A}}_{\varepsilon}+Q^{\varepsilon}\right)^{-1}$ and for the resolvent of the operator $\mathcal{A}_{\varepsilon}=\left(f^{\varepsilon}\right)^{*} \widehat{\mathcal{A}}_{\varepsilon} f^{\varepsilon}$. The results for the resolvent $\left(\mathcal{A}_{\varepsilon}+I\right)^{-1}$ will be used below in Subsection 11.3, which is devoted to the magnetic Schrödinger operator. We use the second versions of the two possible representations (9.5) and (9.6) for $\left(\widehat{\mathcal{A}}_{\varepsilon}+Q^{\varepsilon}\right)^{-1}$ and (9.7) and (9.8) for $\left(\mathcal{A}_{\varepsilon}+I\right)^{-1}$, i.e., we employ (9.6) and (9.8).

Theorem 10.6. 1) Under the assumptions of Theorem $10.5(1)$, let $Q(\mathbf{x})$ be a positive $\Gamma$-periodic function such that $Q, Q^{-1} \in L_{\infty}$. We write $Q(\mathbf{x})$ as $Q(\mathbf{x})=|f(\mathbf{x})|^{-2}$, where $f(\mathbf{x})$ is a $\Gamma$-periodic function (possibly, complex-valued). Let $\mathcal{A}_{\varepsilon}=\left(f^{\varepsilon}\right)^{*} \widehat{\mathcal{A}}_{\varepsilon} f^{\varepsilon}$, and let $\Pi_{\varepsilon}$ be the operator defined by (8.8). We put $\widehat{\mathcal{R}}_{Q}^{0}=\left(\widehat{\mathcal{A}}^{0}+\bar{Q}\right)^{-1}$. Then for $\varepsilon>0$ we have

$$
\begin{aligned}
\left(\widehat{\mathcal{A}}_{\varepsilon}+Q^{\varepsilon}\right)^{-1}= & \widehat{\mathcal{R}}_{Q}^{0}+\varepsilon \widetilde{K}_{Q}(\varepsilon)+\varepsilon^{2} \widetilde{\mathcal{I}}^{\prime}(\varepsilon), \\
\widetilde{K}_{Q}(\varepsilon)= & \sum_{j=1}^{d} \Phi_{j}^{\varepsilon} \partial_{j} \Pi_{\varepsilon} \widehat{\mathcal{R}}_{Q}^{0}-\widehat{\mathcal{R}}_{Q}^{0} \Pi_{\varepsilon} \sum_{j=1}^{d} \partial_{j}\left(\Phi_{j}^{\varepsilon}\right)^{*} \\
& -\widehat{\mathcal{R}}_{Q}^{0}\left(\sum_{j, l, s=1}^{d}\left(a_{j l s}-a_{j l s}^{*}\right) \partial_{j} \partial_{l} \partial_{s}+2 i \sum_{j=1}^{d}\left(\operatorname{Im} \overline{Q \Phi_{j}}\right) \partial_{j}\right) \widehat{\mathcal{R}}_{Q}^{0}, \\
\left(\mathcal{A}_{\varepsilon}+I\right)^{-1}= & \left(f^{\varepsilon}\right)^{-1}\left(\widehat{\mathcal{R}}_{Q}^{0}+\varepsilon \widetilde{K}_{Q}(\varepsilon)\right)\left(\left(f^{\varepsilon}\right)^{*}\right)^{-1}+\varepsilon^{2} \widetilde{\mathcal{Y}}^{\prime}(\varepsilon),
\end{aligned}
$$

and

$$
\left\|\widetilde{\mathcal{I}}^{\prime}(\varepsilon)\right\|_{L_{2}\left(\mathbb{R}^{d}\right) \rightarrow L_{2}\left(\mathbb{R}^{d}\right)} \leq{\widetilde{\mathcal{C}_{1}^{\prime}}}_{1}^{\prime}, \quad\left\|\widetilde{\mathcal{Y}}^{\prime}(\varepsilon)\right\|_{L_{2}\left(\mathbb{R}^{d}\right) \rightarrow L_{2}\left(\mathbb{R}^{d}\right)} \leq{\widetilde{\mathcal{C}_{1}^{\prime}}}^{\prime}\|Q\|_{L_{\infty}} .
$$

The constant $\widetilde{\mathcal{C}_{1}^{\prime}}$ depends only on $\|g\|_{L_{\infty}},\left\|g^{-1}\right\|_{L_{\infty}},\|Q\|_{L_{\infty}},\left\|Q^{-1}\right\|_{L_{\infty}}$, and the parameters of the lattice $\Gamma$.

2) Suppose that the assumptions of Theorem 10.5(2) are satisfied, and that $Q(\mathbf{x})$ is the same as in statement 1$)$. Then for $\varepsilon>0$ we have

$$
\begin{aligned}
\left(\widehat{\mathcal{A}}_{\varepsilon}+Q^{\varepsilon}\right)^{-1}= & \widehat{\mathcal{R}}_{Q}^{0}+\varepsilon \widetilde{K}_{Q}^{\circ}(\varepsilon)+\varepsilon^{2} \widetilde{\mathcal{I}}^{\prime \prime}(\varepsilon), \\
\widetilde{K}_{Q}^{\circ}(\varepsilon)= & \sum_{j=1}^{d} \Phi_{j}^{\varepsilon} \partial_{j} \widehat{\mathcal{R}}_{Q}^{0}-\widehat{\mathcal{R}}_{Q}^{0} \sum_{j=1}^{d} \partial_{j}\left(\Phi_{j}^{\varepsilon}\right)^{*} \\
& -\widehat{\mathcal{R}}_{Q}^{0}\left(\sum_{j, l, s=1}^{d}\left(a_{j l s}-a_{j l s}^{*}\right) \partial_{j} \partial_{l} \partial_{s}+2 i \sum_{j=1}^{d}\left(\operatorname{Im} \overline{Q \Phi_{j}}\right) \partial_{j}\right) \widehat{\mathcal{R}}_{Q}^{0}, \\
\left(\mathcal{A}_{\varepsilon}+I\right)^{-1}= & \left(f^{\varepsilon}\right)^{-1}\left(\widehat{\mathcal{R}}_{Q}^{0}+\varepsilon \widetilde{K}_{Q}^{\circ}(\varepsilon)\right)\left(\left(f^{\varepsilon}\right)^{*}\right)^{-1}+\varepsilon^{2} \widetilde{\mathcal{Y}}^{\prime \prime}(\varepsilon),
\end{aligned}
$$


and

$$
\left\|\widetilde{\mathcal{I}}^{\prime \prime}(\varepsilon)\right\|_{L_{2}\left(\mathbb{R}^{d}\right) \rightarrow L_{2}\left(\mathbb{R}^{d}\right)} \leq \widetilde{\mathcal{C}}_{1}^{\prime \prime}, \quad\left\|\widetilde{\mathcal{Y}}^{\prime \prime}(\varepsilon)\right\|_{L_{2}\left(\mathbb{R}^{d}\right) \rightarrow L_{2}\left(\mathbb{R}^{d}\right)} \leq \widetilde{\mathcal{C}}_{1}^{\prime \prime}\|Q\|_{L_{\infty}}
$$

The constant $\widetilde{\mathcal{C}_{1}^{\prime \prime}}$ depends on $\|g\|_{L_{\infty}},\left\|g^{-1}\right\|_{L_{\infty}},\|Q\|_{L_{\infty}},\left\|Q^{-1}\right\|_{L_{\infty}}$, d, the parameters of the lattice $\Gamma$, and on the norms $\left\|\left[\Phi_{j}\right]\right\|_{H^{1}\left(\mathbb{R}^{d}\right) \rightarrow L_{2}\left(\mathbb{R}^{d}\right)}, j=1, \ldots, d$.

10.6. Particular cases. The case where the corrector is zero is distinguished by Theorems 8.3 and 9.2. Now conditions (3.9) mean that the columns $\mathbf{g}_{j}(\mathbf{x})$ of the matrix $g(\mathbf{x})$ are divergence free:

$$
\operatorname{div} \mathbf{g}_{j}(\mathbf{x})=0, \quad j=1, \ldots, d,
$$

which is equivalent to the fact that $g^{0}=\bar{g}$. Thus, we obtain the following theorem.

Theorem 10.7. Let $\widehat{\mathcal{A}}(g)=\mathbf{D}^{*} g(\mathbf{x}) \mathbf{D}$, where $g(\mathbf{x})$ is a Hermitian $\Gamma$-periodic matrix with, in general, complex entries. Suppose that conditions (10.2) and (10.17) are satisfied. Let $\widehat{\mathcal{A}}^{0}=\widehat{\mathcal{A}}\left(g^{0}\right)$ be the effective operator, let $\widehat{\mathcal{A}_{\varepsilon}}=\widehat{\mathcal{A}}\left(g^{\varepsilon}\right)$, and let $Q(\mathbf{x})>0$ be a $\Gamma$-periodic function such that $Q, Q^{-1} \in L_{\infty}$. Then

$$
\begin{aligned}
\left\|\left(\widehat{\mathcal{A}}_{\varepsilon}+I\right)^{-1}-\left(\widehat{\mathcal{A}}^{0}+I\right)^{-1}\right\|_{L_{2}\left(\mathbb{R}^{d}\right) \rightarrow L_{2}\left(\mathbb{R}^{d}\right)} \leq \widehat{\mathcal{C}}_{2} \varepsilon^{2}, & \varepsilon>0, \\
\left\|\left(\widehat{\mathcal{A}}_{\varepsilon}+Q^{\varepsilon}\right)^{-1}-\left(\widehat{\mathcal{A}}^{0}+\bar{Q}\right)^{-1}\right\|_{L_{2}\left(\mathbb{R}^{d}\right) \rightarrow L_{2}\left(\mathbb{R}^{d}\right)} \leq \mathcal{C}_{2} \varepsilon^{2}, & \varepsilon>0 .
\end{aligned}
$$

The constant $\widehat{\mathcal{C}_{2}}$ depends on $\|g\|_{L_{\infty}},\left\|g^{-1}\right\|_{L_{\infty}}$, and the parameters of the lattice, while $\mathcal{C}_{2}$ depends on the same parameters and on $\|Q\|_{L_{\infty}}$ and $\left\|Q^{-1}\right\|_{L_{\infty}}$.

The case where the third term in (10.15) is zero is distinguished by Proposition 8.4. Now conditions (3.10) mean that the columns $\mathbf{l}_{k}(\mathbf{x}), k=1, \ldots, d$, of the matrix $g(\mathbf{x})^{-1}$ are potential vectors:

$$
\mathbf{l}_{k}(\mathbf{x})=\mathbf{l}_{k}^{0}+\nabla \phi_{k}, \quad \mathbf{l}_{k}^{0} \in \mathbb{C}^{d}, \quad \phi_{k} \in \widetilde{H}^{1}(\Omega), \quad k=1, \ldots, d .
$$

This is equivalent to the relation $g^{0}=\underline{g}$. In this case expression (10.16) for the corrector $\widetilde{K}_{Q}^{\circ}(\varepsilon)$ simplifies. Propositions 8.4 and 9.5 yield the following.

Proposition 10.8. Let $\widehat{\mathcal{A}}(g)=\mathbf{D}^{*} g(\mathbf{x}) \mathbf{D}$, where $g(\mathbf{x})$ is a Hermitian $\Gamma$-periodic matrixvalued function with, in general, complex entries. Suppose that conditions (10.2) and (10.19) are satisfied. Let $\widehat{\mathcal{A}}^{0}=\widehat{\mathcal{A}}\left(g^{0}\right)$ be the effective operator, let $\widehat{\mathcal{A}_{\varepsilon}}=\widehat{\mathcal{A}}\left(g^{\varepsilon}\right)$, and let $\Phi_{j} \in \widetilde{H}^{1}(\Omega)$ be the solution of problem $(10.3), j=1, \ldots, d$. Suppose that $Q(\mathbf{x})>0$ is a $\Gamma$-periodic function such that $Q, Q^{-1} \in L_{\infty}$. Then for $\varepsilon>0$ we have

$$
\begin{aligned}
\left(\widehat{\mathcal{A}}_{\varepsilon}+I\right)^{-1}= & \widehat{\mathcal{R}}^{0}+\varepsilon \sum_{j=1}^{d} \Phi_{j}^{\varepsilon} \partial_{j} \widehat{\mathcal{R}}^{0}-\varepsilon \widehat{\mathcal{R}}^{0} \sum_{j=1}^{d} \partial_{j}\left(\Phi_{j}^{\varepsilon}\right)^{*}+\varepsilon^{2} \widehat{\mathcal{I}}^{\times}(\varepsilon), \\
\left(\widehat{\mathcal{A}}_{\varepsilon}+Q^{\varepsilon}\right)^{-1}= & \widehat{\mathcal{R}}_{Q}^{0}+\varepsilon \sum_{j=1}^{d} \Phi_{j}^{\varepsilon} \partial_{j} \widehat{\mathcal{R}}_{Q}^{0}-\varepsilon \widehat{\mathcal{R}}_{Q}^{0} \sum_{j=1}^{d} \partial_{j}\left(\Phi_{j}^{\varepsilon}\right)^{*} \\
& -\varepsilon \widehat{\mathcal{R}}_{Q}^{0}\left(2 i \sum_{j=1}^{d}\left(\operatorname{Im} \overline{Q \Phi_{j}}\right) \partial_{j}\right) \widehat{\mathcal{R}}_{Q}^{0}+\varepsilon^{2} \widetilde{\mathcal{I}}^{\prime \prime}(\varepsilon) .
\end{aligned}
$$

Here $\widehat{\mathcal{R}}^{0}=\left(\widehat{\mathcal{A}}^{0}+I\right)^{-1}, \widehat{\mathcal{R}}_{Q}^{0}=\left(\widehat{\mathcal{A}}^{0}+\bar{Q}\right)^{-1}$, and

$$
\left\|\widehat{\mathcal{I}}^{\times}(\varepsilon)\right\|_{L_{2}\left(\mathbb{R}^{d}\right) \rightarrow L_{2}\left(\mathbb{R}^{d}\right)} \leq \widehat{\mathcal{C}}_{1}^{\times}, \quad\left\|\widetilde{\mathcal{I}}^{\prime \prime}(\varepsilon)\right\|_{L_{2}\left(\mathbb{R}^{d}\right) \rightarrow L_{2}\left(\mathbb{R}^{d}\right)} \leq \widetilde{\mathcal{C}}_{1}^{\prime \prime} .
$$

The constant $\widehat{\mathcal{C}_{1}^{\times}}$depends on $\|g\|_{L_{\infty}},\left\|g^{-1}\right\|_{L_{\infty}}, d$, and the parameters of the lattice, while $\widetilde{\mathcal{C}}_{1}^{\prime \prime}$ depends on the same parameters and on $\|Q\|_{L_{\infty}}$ and $\left\|Q^{-1}\right\|_{L_{\infty}}$.

We recall that if the matrix $g$ has real entries, then the representations (10.4) and (10.6) are always true. 


\section{§11. The PERIOdIC Schrödinger Operator}

11.1. Preliminaries. Factorization. (See $[\overline{\mathrm{BSu} 2}, \S 6.1]$.) In the space $L_{2}\left(\mathbb{R}^{d}\right), d \geq 1$, we consider the periodic Schrödinger operator (with metric)

$$
\mathcal{H}=\mathbf{D}^{*} \mathfrak{g}(\mathbf{x}) \mathbf{D}+p(\mathbf{x}), \quad \mathbf{x} \in \mathbb{R}^{d},
$$

where the $(d \times d)$-matrix-valued function $\mathfrak{g}(\mathbf{x})$ with real entries and the real-valued potential $p(\mathbf{x})$ are $\Gamma$-periodic and satisfy

$$
\begin{gathered}
\mathfrak{g}(\mathbf{x})>0, \quad \mathfrak{g}, \mathfrak{g}^{-1} \in L_{\infty} \\
p \in L_{s}(\Omega), \begin{cases}2 s>d & \text { for } d \geq 2, \\
s=1 & \text { for } d=1\end{cases}
\end{gathered}
$$

Adding an appropriate constant to $p(\mathbf{x})$, we may assume that the point $\lambda=0$ is the bottom of the spectrum of $\mathcal{H}$.

Let $\omega \in \widetilde{H}^{1}(\Omega)$ be a (weak) periodic solution of the equation

$$
\mathbf{D}^{*} \mathfrak{g}(\mathbf{x}) \mathbf{D} \omega+p(\mathbf{x}) \omega=0 .
$$

Under conditions (11.2) and (11.3), there exists a (strictly) positive solution $\omega$ such that $\omega \in C^{\alpha}$ for some $\alpha>0$. We fix the choice of $\omega$ by the condition

$$
\int_{\Omega} \omega^{2}(\mathbf{x}) d \mathbf{x}=|\Omega| .
$$

The operator (11.1) reshapes to the form

$$
\mathcal{H}=\omega^{-1} \mathbf{D}^{*} \omega^{2} \mathfrak{g} \mathbf{D} \omega^{-1} .
$$

Thus, the operator $\mathcal{H}$ is reduced to the form (2.6):

$$
\mathcal{H}=\mathcal{A}(g, f), \quad g=\omega^{2} \mathfrak{g}, \quad f=\omega^{-1},
$$

with $n=1, m=d, b(\mathbf{D})=\mathbf{D}$.

Remark 11.1. We can view (11.5) as the definition of the operator $\mathcal{H}$, assuming that $\omega(\mathbf{x})$ is an arbitrary $\Gamma$-periodic function such that

$$
\omega(\mathbf{x})>0, \quad \omega, \omega^{-1} \in L_{\infty} .
$$

We take this definition as the initial one. The form (11.1) can be recovered by putting $p=-\omega^{-1}\left(\mathbf{D}^{*} \mathfrak{g} \mathbf{D} \omega\right)$. The resulting potential $p(\mathbf{x})$ may happen to be strongly singular.

11.2. The homogenization problem for $\mathcal{H}_{\varepsilon}$. Now we consider the operator

$$
\mathcal{H}_{\varepsilon}=\left(\omega^{\varepsilon}\right)^{-1} \mathbf{D}^{*}\left(\omega^{\varepsilon}\right)^{2} \mathfrak{g}^{\varepsilon} \mathbf{D}\left(\omega^{\varepsilon}\right)^{-1}=\left(\omega^{\varepsilon}\right)^{-1} \mathbf{D}^{*} g^{\varepsilon} \mathbf{D}\left(\omega^{\varepsilon}\right)^{-1}
$$

with rapidly oscillating coefficients. In the form (11.1), the operator (11.7) can be written as

$$
\mathcal{H}_{\varepsilon}=\mathbf{D}^{*} \mathfrak{g}^{\varepsilon}(\mathbf{x}) \mathbf{D}+\varepsilon^{-2} p^{\varepsilon}(\mathbf{x}) .
$$

We are interested in the behavior of the resolvent $\left(\mathcal{H}_{\varepsilon}+I\right)^{-1}$ for small $\varepsilon$. Let $\widehat{\mathcal{A}}(g)=$ $\mathbf{D}^{*} \omega^{2} \mathfrak{g} \mathbf{D}=\mathbf{D}^{*} g \mathbf{D}$, let $g^{0}$ be the effective matrix for the operator $\widehat{\mathcal{A}}(g)$, and let $\widehat{\mathcal{A}}^{0}=$ $\widehat{\mathcal{A}}\left(g^{0}\right)$. Proposition 9.4 is applicable. Now $Q(\mathbf{x})=\omega^{2}(\mathbf{x})$, and $\bar{Q}=1$ by (11.4). By the above arguments, Proposition 9.4 implies the following statement (cf. Theorem 10.2).

Theorem 11.2. Let $\mathfrak{g}(\mathbf{x})$ be a $\Gamma$-periodic $(d \times d)$-matrix-valued function with real entries and satisfying (11.2), let $\omega(\mathbf{x})$ be a $\Gamma$-periodic function satisfying (11.6) and (11.4), and let $\mathcal{H}_{\varepsilon}$ be the operator defined by (11.7). Put $g(\mathbf{x}):=\omega^{2}(\mathbf{x}) \mathfrak{g}(\mathbf{x})$, and denote by $g^{0}$ the 
effective matrix for the operator $\mathbf{D}^{*} g \mathbf{D}$. Let $\widehat{\mathcal{A}}^{0}=\mathbf{D}^{*} g^{0} \mathbf{D}$, and let $\Phi_{j} \in \widetilde{H}^{1}(\Omega)$ be the solution of problem (10.3), $j=1, \ldots, d$. Then for $\varepsilon>0$ we have

$$
\left(\mathcal{H}_{\varepsilon}+I\right)^{-1}=\omega^{\varepsilon}\left(\widehat{\mathcal{R}}^{0}+\varepsilon \sum_{j=1}^{d} \Phi_{j}^{\varepsilon} \partial_{j} \widehat{\mathcal{R}}^{0}-\varepsilon \widehat{\mathcal{R}}^{0} \sum_{j=1}^{d} \partial_{j} \Phi_{j}^{\varepsilon}\right) \omega^{\varepsilon}+\varepsilon^{2} \mathcal{Y}^{\times}(\varepsilon),
$$

where $\widehat{\mathcal{R}}^{0}=\left(\widehat{\mathcal{A}}^{0}+I\right)^{-1}$, and

$$
\left\|\mathcal{Y}^{\times}(\varepsilon)\right\|_{L_{2}\left(\mathbb{R}^{d}\right) \rightarrow L_{2}\left(\mathbb{R}^{d}\right)} \leq \mathcal{C}_{1}^{\times}\|\omega\|_{L_{\infty}}^{2} .
$$

Here $\mathcal{C}_{1}^{\times}$is the constant occurring in (10.7) and depending only on $\|\mathfrak{g}\|_{L_{\infty}},\left\|\mathfrak{g}^{-1}\right\|_{L_{\infty}}$, $\|\omega\|_{L_{\infty}},\left\|\omega^{-1}\right\|_{L_{\infty}}, d$, and the parameters of the lattice $\Gamma$.

The case where the corrector in (11.8) is zero is distinguished by Theorem 9.2. We obtain the following theorem.

Theorem 11.3. Under the assumptions of Theorem 11.2, suppose that the columns of the matrix $g(\mathbf{x})=\omega^{2}(\mathbf{x}) \mathfrak{g}(\mathbf{x})$ satisfy (10.17). Then

$$
\left\|\left(\mathcal{H}_{\varepsilon}+I\right)^{-1}-\omega^{\varepsilon}\left(\widehat{\mathcal{A}}^{0}+I\right)^{-1} \omega^{\varepsilon}\right\|_{L_{2}\left(\mathbb{R}^{d}\right) \rightarrow L_{2}\left(\mathbb{R}^{d}\right)} \leq \mathcal{C}_{3} \varepsilon^{2}, \quad \varepsilon>0 .
$$

The constant $\mathcal{C}_{3}$ depends only on $\|\mathfrak{g}\|_{L_{\infty}},\left\|\mathfrak{g}^{-1}\right\|_{L_{\infty}},\|\omega\|_{L_{\infty}},\left\|\omega^{-1}\right\|_{L_{\infty}}$, and the parameters of the lattice $\Gamma$.

Remark 11.4. If we write the operator $\mathcal{H}$ in the form (11.1) under conditions (11.3), then the norms $\|\omega\|_{L_{\infty}}$ and $\left\|\omega^{-1}\right\|_{L_{\infty}}$ are dominated by a constant depending only on $\|\mathfrak{g}\|_{L_{\infty}}$, $\left\|\mathfrak{g}^{-1}\right\|_{L_{\infty}}, d, \Omega$, and $\|p\|_{L_{s}(\Omega)}$. Then the right-hand side of (11.9) and the constant $\mathcal{C}_{3}$ in (11.10) depend on $\|\mathfrak{g}\|_{L_{\infty}},\left\|\mathfrak{g}^{-1}\right\|_{L_{\infty}}, d,\|p\|_{L_{s}(\Omega)}$, and the parameters of the lattice.

Now we consider approximation of the generalized resolvent $\left(\mathcal{H}_{\varepsilon}+\mathfrak{Q}^{\varepsilon}\right)^{-1}$. Here $\mathfrak{Q}(\mathbf{x})$ is a $\Gamma$-periodic positive function such that $\mathfrak{Q}, \mathfrak{Q}^{-1} \in L_{\infty}$. Proposition 9.9 applies.

Theorem 11.5. Under the assumptions of Theorem 11.2 , let $\mathfrak{Q}(\mathbf{x})$ be a $\Gamma$-periodic positive function such that $\mathfrak{Q}, \mathfrak{Q}^{-1} \in L_{\infty}$. Let $Q_{*}(\mathbf{x})=\omega^{2}(\mathbf{x}) \mathfrak{Q}(\mathbf{x})$. Then for $\varepsilon>0$ we have

$$
\left(\mathcal{H}_{\varepsilon}+\mathfrak{Q}^{\varepsilon}\right)^{-1}=\omega^{\varepsilon}\left(\widehat{\mathcal{R}}_{Q_{*}}^{0}+\varepsilon \sum_{j=1}^{d} \Phi_{j}^{\varepsilon} \partial_{j} \widehat{\mathcal{R}}_{Q_{*}}^{0}-\varepsilon \widehat{\mathcal{R}}_{Q_{*}}^{0} \sum_{j=1}^{d} \partial_{j} \Phi_{j}^{\varepsilon}\right) \omega^{\varepsilon}+\varepsilon^{2} \mathcal{I}_{*}^{\times}(\varepsilon),
$$

where $\widehat{\mathcal{R}}_{Q_{*}}^{0}=\left(\widehat{\mathcal{A}}^{0}+\overline{Q_{*}}\right)^{-1}$, and

$$
\left\|\mathcal{I}_{*}^{\times}(\varepsilon)\right\|_{L_{2}\left(\mathbb{R}^{d}\right) \rightarrow L_{2}\left(\mathbb{R}^{d}\right)} \leq \mathcal{C}^{\times} .
$$

Here $\mathcal{C}^{\times}$is a constant depending only on $\|\mathfrak{g}\|_{L_{\infty}},\left\|\mathfrak{g}^{-1}\right\|_{L_{\infty}},\|\omega\|_{L_{\infty}},\left\|\omega^{-1}\right\|_{L_{\infty}},\|\mathfrak{Q}\|_{L_{\infty}}$, $\left\|\mathfrak{Q}^{-1}\right\|_{L_{\infty}}, d$, and the parameters of the lattice $\Gamma$.

Finally, Theorem 9.7 implies the following statement.

Theorem 11.6. Under the assumptions of Theorem 11.5, suppose that the columns of the matrix $g(\mathbf{x})=\omega^{2}(\mathbf{x}) \mathfrak{g}(\mathbf{x})$ satisfy (10.17). Then

$$
\left\|\left(\mathcal{H}_{\varepsilon}+\mathfrak{Q}^{\varepsilon}\right)^{-1}-\omega^{\varepsilon}\left(\widehat{\mathcal{A}}^{0}+\overline{Q_{*}}\right)^{-1} \omega^{\varepsilon}\right\|_{L_{2}\left(\mathbb{R}^{d}\right) \rightarrow L_{2}\left(\mathbb{R}^{d}\right)} \leq \mathcal{C}_{3}^{*} \varepsilon^{2}, \quad \varepsilon>0 .
$$

The constant $\mathcal{C}_{3}^{*}$ depends on $\|\mathfrak{g}\|_{L_{\infty}},\left\|\mathfrak{g}^{-1}\right\|_{L_{\infty}},\|\omega\|_{L_{\infty}},\left\|\omega^{-1}\right\|_{L_{\infty}},\|\mathfrak{Q}\|_{L_{\infty}},\left\|\mathfrak{Q}^{-1}\right\|_{L_{\infty}}$, and the parameters of the lattice $\Gamma$.

Clearly, for $g=\mathbf{1}$, Theorems 11.3 and 11.6 are not informative.

Remark 11.7. In terms of potentials, the operator $\mathcal{H}_{\varepsilon}+\mathfrak{Q}^{\varepsilon}$ takes the form $\mathbf{D}^{*} \mathfrak{g}^{\varepsilon} \mathbf{D}+$ $\varepsilon^{-2} p^{\varepsilon}+\mathfrak{Q}^{\varepsilon}$. If $\omega=1$ (i.e., $p=0$ ), Theorems 11.5 and 11.6 are contained in Theorems 10.6 and 10.7 , respectively. 
11.3. The magnetic Schrödinger operator. In the space $L_{2}\left(\mathbb{R}^{d}\right), d \geq 2$, we consider the periodic magnetic Schrödinger operator $\mathcal{M}$ with metric $\mathfrak{g}(\mathbf{x})$, magnetic potential $\mathbf{A}(\mathbf{x})$, and electric potential $V(\mathbf{x})$ :

$$
\mathcal{M}=(\mathbf{D}-\mathbf{A}(\mathbf{x}))^{*} \mathfrak{g}(\mathbf{x})(\mathbf{D}-\mathbf{A}(\mathbf{x}))+V(\mathbf{x}) .
$$

Here $\mathfrak{g}(\mathbf{x})$ is a $\Gamma$-periodic $(d \times d)$-matrix-valued function with real entries and satisfying conditions (11.2). Next, if $d \geq 3$, we assume that

$$
\mathfrak{g} \in C^{\alpha}, \quad 0<\alpha<1, \quad d \geq 3,
$$

for some $\alpha \in(0,1)$. The vector-valued potential $\mathbf{A}(\mathbf{x})$ and the scalar-valued potential $V(\mathbf{x})$ are real and $\Gamma$-periodic. Suppose that

$$
\mathbf{A} \in L_{2 s}(\Omega), \quad V \in L_{s}(\Omega), \quad 2 s>d .
$$

Adding an appropriate constant to $V(\mathbf{x})$, we may assume that the bottom of the spectrum of $\mathcal{M}$ is the point $\lambda=0$.

In the recent paper [Sh2], Shterenberg showed that, under the above assumptions and for sufficiently small (in the $L_{2 s}(\Omega)$-norm) magnetic potential $\mathbf{A}$, the operator $\mathcal{M}$ admits a factorization appropriate for our goals. We describe this factorization. Let $\mathcal{M}(\mathbf{k})$ be the operators in $L_{2}(\Omega)$ that arise in the direct integral decomposition for $\mathcal{M}$. The condition $\inf \operatorname{spec} \mathcal{M}=0$ means that, for some $\mathbf{k}_{0} \in \widetilde{\Omega}$, the point $\lambda=0$ is an eigenvalue of the operator $\mathcal{M}\left(\mathbf{k}_{0}\right)$. If the magnetic potential is sufficiently small, then such a point $\mathbf{k}_{0} \in \widetilde{\Omega}$ is unique and the eigenvalue $\lambda=0$ is simple. Let $\phi(\mathbf{x})$ be the corresponding eigenfunction normalized by the condition

$$
\int_{\Omega}|\phi(\mathbf{x})|^{2} d \mathbf{x}=|\Omega|
$$

(the phase factor does not matter). Then $\phi \in \widetilde{H}^{1}(\Omega), \mathcal{M}\left(\mathbf{k}_{0}\right) \phi=0$, and

$$
\phi, \phi^{-1} \in L_{\infty} \text {. }
$$

Moreover, as was mentioned in [Sh2],

$$
\phi \in \widetilde{W}_{2 s}^{1}(\Omega), \quad 2 s>d,
$$

with the same exponent $s$ as in (11.13).

We introduce the notation

$$
\widetilde{\mathcal{M}}=\left[e^{-i\left\langle\mathbf{k}_{0}, \cdot\right\rangle}\right] \mathcal{M}\left[e^{i\left\langle\mathbf{k}_{0}, \cdot\right\rangle}\right],
$$

where $\left[e^{ \pm i\left\langle\mathbf{k}_{0}, \cdot\right\rangle}\right]$ is the operator of multiplication by the function $e^{ \pm i\left\langle\mathbf{k}_{0}, \mathbf{x}\right\rangle}$ in $L_{2}\left(\mathbb{R}^{d}\right)$. By [Sh2, Theorems 2.7, 2.8], if the norm $\|\mathbf{A}\|_{L_{2 s}(\Omega)}$ is sufficiently small, then the periodic operator $\widetilde{\mathcal{M}}$ admits the following factorization:

$$
\widetilde{\mathcal{M}}=\left(\phi(\mathbf{x})^{*}\right)^{-1} \mathbf{D}^{*} g(\mathbf{x}) \mathbf{D}(\phi(\mathbf{x}))^{-1} .
$$

Relation (11.18) is similar to the factorization (11.5) for the Schrödinger operator $\mathcal{H}$, but now the Hermitian matrix $g(\mathbf{x})$ has complex entries and the function $\phi(\mathbf{x})$ is complexvalued. Here, $g(\mathbf{x})$ is $\Gamma$-periodic and such that

$$
g(\mathbf{x})>0, \quad g, g^{-1} \in L_{\infty} .
$$

This matrix has the form

$$
g(\mathbf{x})=\mathfrak{g}(\mathbf{x})|\phi(\mathbf{x})|^{2}+i g_{2}(\mathbf{x}),
$$

where the $\Gamma$-periodic antisymmetric matrix $g_{2}(\mathbf{x})$ with real entries satisfies the equation

$$
\left(\operatorname{div} g_{2}(\mathbf{x})\right)^{t}=-2|\phi(\mathbf{x})|^{2} \mathfrak{g}(\mathbf{x})\left(\mathbf{A}(\mathbf{x})-\mathbf{k}_{0}\right)+2 \operatorname{Im}\left(\phi(\mathbf{x})^{*} \mathfrak{g}(\mathbf{x}) \nabla \phi(\mathbf{x})\right) .
$$


Now we consider the homogenization problem for the operator $\mathcal{M}$. We put

$$
\widetilde{\mathcal{M}}_{\varepsilon}=\left(\left(\phi^{\varepsilon}\right)^{*}\right)^{-1} \mathbf{D}^{*} g^{\varepsilon} \mathbf{D}\left(\phi^{\varepsilon}\right)^{-1}, \quad \mathcal{M}_{\varepsilon}=\left[e^{i \varepsilon^{-1}\left\langle\mathbf{k}_{0}, \cdot\right\rangle}\right] \widetilde{\mathcal{M}}_{\varepsilon}\left[e^{-i \varepsilon^{-1}\left\langle\mathbf{k}_{0}, \cdot\right\rangle}\right]
$$

In the initial terms, we have

$$
\mathcal{M}_{\varepsilon}=\left(\mathbf{D}-\varepsilon^{-1} \mathbf{A}^{\varepsilon}\right)^{*} \mathfrak{g}^{\varepsilon}\left(\mathbf{D}-\varepsilon^{-1} \mathbf{A}^{\varepsilon}\right)+\varepsilon^{-2} V^{\varepsilon} .
$$

The behavior of the resolvent $\left(\widetilde{\mathcal{M}}_{\varepsilon}+I\right)^{-1}$ is controlled in Theorem 10.6(2). Indeed, now $f(\mathbf{x})=\phi(\mathbf{x})^{-1}, Q(\mathbf{x})=|\phi(\mathbf{x})|^{2}$, and $\bar{Q}=1$ by (11.14). Let $g^{0}$ be the effective matrix for the operator $\widehat{\mathcal{A}}=\mathbf{D}^{*} g \mathbf{D}$, and let $\widehat{\mathcal{A}}^{0}=\mathbf{D}^{*} g^{0} \mathbf{D}$. Then $\widehat{\mathcal{R}}_{Q}^{0}=\left(\widehat{\mathcal{A}}^{0}+\bar{Q}\right)^{-1}=$ $\left(\widehat{\mathcal{A}}^{0}+I\right)^{-1}=\widehat{\mathcal{R}}^{0}$. Next, the matrix $g_{2}(\mathbf{x})=\operatorname{Im} g(\mathbf{x})$ satisfies (10.14) with $q=2 s>d$, because the function on the right-hand side of (11.20) belongs to $L_{2 s}(\Omega)$, by conditions (11.2), (11.13), (11.15), and (11.16). Thus, the assumptions of Theorem 10.6(2) are satisfied. Using the identity

$$
\left(\mathcal{M}_{\varepsilon}+I\right)^{-1}=\left[e^{i \varepsilon^{-1}\left\langle\mathbf{k}_{0}, \cdot\right\rangle}\right]\left(\widetilde{\mathcal{M}}_{\varepsilon}+I\right)^{-1}\left[e^{-i \varepsilon^{-1}\left\langle\mathbf{k}_{0}, \cdot\right\rangle}\right],
$$

we obtain the following result.

Theorem 11.8. Let $\mathcal{M}$ be the operator (11.11) with $\Gamma$-periodic real coefficients satisfying conditions (11.2), (11.12), and (11.13). Suppose that the norm $\|\mathbf{A}\|_{L_{2 s}(\Omega)}$ is so small that the factorization (11.18) for the operator (11.17) is valid, where the $\Gamma$-periodic matrix $g(\mathbf{x})$ and the function $\phi(\mathbf{x})$ satisfy conditions (11.19) and (11.14), (11.15). Let $\mathcal{M}_{\varepsilon}$ be the operator defined by (11.21), let $g^{0}$ be the effective matrix for the operator $\mathbf{D}^{*} g(\mathbf{x}) \mathbf{D}$, and let $\widehat{\mathcal{A}}^{0}=\mathbf{D}^{*} g^{0} \mathbf{D}$. Let $\Phi_{j} \in \widetilde{H}^{1}(\Omega)$ be the solution of problem $(10.3), j=1, \ldots, d$, let $Q(\mathbf{x})=|\phi(\mathbf{x})|^{2}$, and let $a_{j l s}$ be the coefficients defined by formulas (10.12), $j, l, s=$ $1, \ldots, d$. Then for $\varepsilon>0$ we have

$$
\begin{aligned}
\left(\mathcal{M}_{\varepsilon}+I\right)^{-1}= & {\left[e^{i \varepsilon^{-1}\left\langle\mathbf{k}_{0}, \cdot\right\rangle}\right] \phi^{\varepsilon}\left(\widehat{\mathcal{R}}^{0}+\varepsilon \sum_{j=1}^{d} \Phi_{j}^{\varepsilon} \partial_{j} \widehat{\mathcal{R}}^{0}-\varepsilon \widehat{\mathcal{R}}^{0} \sum_{j=1}^{d} \partial_{j}\left(\Phi_{j}^{\varepsilon}\right)^{*}\right.} \\
& \left.-\varepsilon \widehat{\mathcal{R}}^{0}\left(\sum_{j, l, s=1}^{d}\left(a_{j l s}-a_{j l s}^{*}\right) \partial_{j} \partial_{l} \partial_{s}+2 i \sum_{j=1}^{d} \operatorname{Im}\left(\overline{Q \Phi_{j}}\right) \partial_{j}\right) \widehat{\mathcal{R}}^{0}\right) \\
& \times\left(\phi^{\varepsilon}\right)^{*}\left[e^{-i \varepsilon^{-1}\left\langle\mathbf{k}_{0}, \cdot\right\rangle}\right]+\varepsilon^{2} \mathcal{Y}(\varepsilon),
\end{aligned}
$$

where $\widehat{\mathcal{R}}^{0}=\left(\widehat{\mathcal{A}}^{0}+I\right)^{-1}$. The norm $\|\mathcal{Y}(\varepsilon)\|_{L_{2}\left(\mathbb{R}^{d}\right) \rightarrow L_{2}\left(\mathbb{R}^{d}\right)}$ is uniformly bounded.

The case where the corrector in (11.22) is zero is distinguished by Theorem 10.7. Applying that theorem, we arrive at the following result.

Theorem 11.9. Under the assumptions of Theorem 11.8, suppose that the columns of the matrix $g(\mathbf{x})$ are divergence free (i.e., (10.17) is valid). Then

$$
\left\|\left(\mathcal{M}_{\varepsilon}+I\right)^{-1}-\left[e^{i \varepsilon^{-1}\left\langle\mathbf{k}_{0}, \cdot\right\rangle}\right]\left(\phi^{\varepsilon}\right)\left(\widehat{\mathcal{A}}^{0}+I\right)^{-1}\left(\phi^{\varepsilon}\right)^{*}\left[e^{-i \varepsilon^{-1}\left\langle\mathbf{k}_{0}, \cdot\right\rangle}\right]\right\|_{L_{2}\left(\mathbb{R}^{d}\right) \rightarrow L_{2}\left(\mathbb{R}^{d}\right)} \leq C \varepsilon^{2} .
$$

We note that, under the conditions of Theorems 11.8 and 11.9, it is difficult to control the constants in the estimates explicitly.

Remark 11.10. If the columns of the matrix $g^{-1}$ are potential vectors (i.e., (10.19) is satisfied), then Proposition 9.5 is applicable. By this proposition, the term in (11.22) that involves a triple sum vanishes. 
Remark 11.11. In [BSu2, the magnetic Schrödinger operator was not considered. After the publication of the paper [Sh2, it became possible to treat this operator. Under the conditions of Theorem 11.8, the general results of [BSu2] directly yield the estimate

$$
\left\|\left(\mathcal{M}_{\varepsilon}+I\right)^{-1}-\left[e^{i \varepsilon^{-1}\left\langle\mathbf{k}_{0}, \cdot\right\rangle}\right]\left(\phi^{\varepsilon}\right)\left(\widehat{\mathcal{A}}^{0}+I\right)^{-1}\left(\phi^{\varepsilon}\right)^{*}\left[e^{-i \varepsilon^{-1}\left\langle\mathbf{k}_{0}, \cdot\right\rangle}\right]\right\|_{L_{2}\left(\mathbb{R}^{d}\right) \rightarrow L_{2}\left(\mathbb{R}^{d}\right)} \leq C \varepsilon .
$$

The more precise estimate (11.23) is a consequence of the divergence free conditions (10.17).

Remark 11.12. In [Sh1] it was shown that, in general, without the smallness condition on the potential A, the required factorization for the magnetic Schrödinger operator fails.

\section{§12. The two-dimensional periodic Pauli operator}

12.1. The operators $\widehat{\mathcal{B}}_{ \pm}$. The examples to be considered in Subsections 12.1 and 12.2 (cf. [BSu2, Subsection 5.1.3]) are of a preliminary nature. They will be useful in the discussion of the two-dimensional Pauli operator in Subsections 12.3-12.5. However, these examples are of interest in themselves.

Within Subsection 12.1, all formulas and statements should be read independently for the upper and the lower indices.

Suppose $d=2$ and $m=n=1$. Let $\omega_{ \pm}(\mathbf{x})$ be a $\Gamma$-periodic function such that

$$
\omega_{ \pm}(\mathbf{x})>0, \quad \omega_{ \pm}, \omega_{ \pm}^{-1} \in L_{\infty} .
$$

We consider the operator

$$
\widehat{\mathcal{B}}_{ \pm}=\widehat{\mathcal{B}}_{ \pm}\left(\omega_{ \pm}^{2}\right)=\partial_{ \pm} \omega_{ \pm}^{2} \partial_{\mp},
$$

where $\partial_{ \pm}=D_{1} \pm i D_{2}$. (The operators $\widehat{\mathcal{B}}_{+}$and $\widehat{\mathcal{B}}_{-}$are of the same type, and it is convenient to consider them in parallel.) The operator $\widehat{\mathcal{B}}_{ \pm}\left(\omega_{ \pm}^{2}\right)$ is an operator of the form $\widehat{\mathcal{A}}(g)$ with $g=g_{ \pm}=\omega_{ \pm}^{2}, b(\mathbf{D})=D_{1} \mp i D_{2}$. Obviously, now we have $\alpha_{0}=\alpha_{1}=1$. Since $m=n=1$, the effective constant $g_{ \pm}^{0}$ for the operator $\widehat{\mathcal{B}}_{ \pm}$is equal to $\underline{g_{ \pm}}$, i.e.,

$$
g_{ \pm}^{0}=\underline{\left(\omega_{ \pm}^{2}\right)}=\left(|\Omega|^{-1} \int_{\Omega} \omega_{ \pm}(\mathbf{x})^{-2} d \mathbf{x}\right)^{-1} .
$$

Then the effective operator $\widehat{\mathcal{B}}_{ \pm}^{0}$ for the operator $\widehat{\mathcal{B}}_{ \pm}$coincides with $-g_{ \pm}^{0} \Delta$ :

$$
\widehat{\mathcal{B}}_{ \pm}^{0}=-g_{ \pm}^{0} \Delta \text {. }
$$

We consider the solution $v_{ \pm} \in \widetilde{H}^{1}(\Omega)$ of the equation

$$
\partial_{ \pm} \omega_{ \pm}^{2}(\mathbf{x})\left(\partial_{\mp} v_{ \pm}+1\right)=0, \quad \int_{\Omega} v_{ \pm}(\mathbf{x}) d \mathbf{x}=0
$$

By Remark 3.5, the function $\omega_{ \pm}^{2}\left(\partial_{\mp} v_{ \pm}+1\right)$ is a constant equal to $g_{ \pm}^{0}=\underline{\left(\omega_{ \pm}^{2}\right)}$. Therefore, $v_{ \pm}$is the solution of the equation

$$
\partial_{\mp} v_{ \pm}=g_{ \pm}^{0} \omega_{ \pm}(\mathbf{x})^{-2}-1, \quad \int_{\Omega} v_{ \pm}(\mathbf{x}) d \mathbf{x}=0 .
$$

The role of the "matrix" $\Lambda(\mathbf{x})$ for the operator $\widehat{\mathcal{B}}_{ \pm}$is played by the function $v_{ \pm}(\mathbf{x})$.

Now, we consider the operator

$$
\widehat{\mathcal{B}}_{ \pm, \varepsilon}=\widehat{\mathcal{B}}_{ \pm}\left(\left(\omega_{ \pm}^{\varepsilon}\right)^{2}\right) .
$$

Since $g_{ \pm}^{0}=\underline{g_{ \pm}}$, we can apply Proposition 8.4; this yields the following statement. 
Proposition 12.1 $( \pm)$. Let $\omega_{ \pm}(\mathbf{x})$ be a $\Gamma$-periodic function in $\mathbb{R}^{2}$ satisfying conditions $(12.1 \pm)$. Let $\widehat{\mathcal{B}}_{ \pm, \varepsilon}$ be the operator defined by $(12.2 \pm)$ and $(12.6 \pm)$; next, let $g_{ \pm}^{0}$ be the constant defined by $(12.3 \pm)$, and let $v_{ \pm} \in \widetilde{H}^{1}(\Omega)$ be the solution of problem (12.5 \pm ). Then for $\varepsilon>0$ we have

$$
\left(\widehat{\mathcal{B}}_{ \pm, \varepsilon}+I\right)^{-1}=\widehat{\mathcal{R}}_{ \pm}^{0}+\varepsilon v_{ \pm}^{\varepsilon} \partial_{\mp} \widehat{\mathcal{R}}_{ \pm}^{0}+\varepsilon \widehat{\mathcal{R}}_{ \pm}^{0} \partial_{ \pm}\left(v_{ \pm}^{\varepsilon}\right)^{*}+\varepsilon^{2} \widehat{\mathcal{I}}_{ \pm}(\varepsilon)
$$

where $\widehat{\mathcal{R}}_{ \pm}^{0}=\left(-g_{ \pm}^{0} \Delta+I\right)^{-1}$, and

$$
\left\|\widehat{\mathcal{I}}_{ \pm}(\varepsilon)\right\|_{L_{2}\left(\mathbb{R}^{2}\right) \rightarrow L_{2}\left(\mathbb{R}^{2}\right)} \leq \widehat{\mathcal{C}}_{ \pm}
$$

The constant $\widehat{\mathcal{C}_{ \pm}}$depends only on $\left\|\omega_{ \pm}\right\|_{L_{\infty}},\left\|\omega_{ \pm}^{-1}\right\|_{L_{\infty}}$, and on the lattice $\Gamma$.

We pass to the generalized resolvent $\left(\widehat{\mathcal{B}}_{ \pm, \varepsilon}+Q_{ \pm}^{\varepsilon}\right)^{-1}$, where $Q_{ \pm}(\mathbf{x})$ is a $\Gamma$-periodic function such that

$$
Q_{ \pm}(\mathbf{x})>0, \quad Q_{ \pm}, Q_{ \pm}^{-1} \in L_{\infty} .
$$

Applying Proposition 9.5, we obtain the following.

Proposition 12.2( \pm ). Under the assumptions of Proposition $12.1( \pm)$, let $Q_{ \pm}(\mathbf{x})$ be a $\Gamma$-periodic function satisfying (12.7 \pm ). Then for $\varepsilon>0$ we have

$$
\begin{aligned}
\left(\widehat{\mathcal{B}}_{ \pm, \varepsilon}+Q_{ \pm}^{\varepsilon}\right)^{-1}= & \widehat{\mathcal{R}}_{ \pm, Q_{ \pm}}^{0}+\varepsilon v_{ \pm}^{\varepsilon} \partial_{\mp} \widehat{\mathcal{R}}_{ \pm, Q_{ \pm}}^{0}+\varepsilon \widehat{\mathcal{R}}_{ \pm, Q_{ \pm}}^{0} \partial_{ \pm}\left(v_{ \pm}^{\varepsilon}\right)^{*} \\
& -\varepsilon \widehat{\mathcal{R}}_{ \pm, Q_{ \pm}}^{0}\left(2 D_{1}\left(\operatorname{Re} \overline{Q_{ \pm} v_{ \pm}}\right) \pm 2 D_{2}\left(\operatorname{Im} \overline{Q_{ \pm} v_{ \pm}}\right)\right) \widehat{\mathcal{R}}_{ \pm, Q_{ \pm}}^{0}+\varepsilon^{2} \widetilde{\mathcal{I}}_{ \pm}(\varepsilon),
\end{aligned}
$$

where $\widehat{\mathcal{R}}_{ \pm, Q_{ \pm}}^{0}=\left(-g_{ \pm}^{0} \Delta+\overline{Q_{ \pm}}\right)^{-1}$, and

$$
\left\|\widetilde{\mathcal{I}}_{ \pm}(\varepsilon)\right\|_{L_{2}\left(\mathbb{R}^{2}\right) \rightarrow L_{2}\left(\mathbb{R}^{2}\right)} \leq \widetilde{\mathcal{C}}_{ \pm}^{\prime \prime}
$$

The constant $\widetilde{\mathcal{C}}_{ \pm}^{\prime \prime}$ depends only on $\left\|\omega_{ \pm}\right\|_{L_{\infty}},\left\|\omega_{ \pm}^{-1}\right\|_{L_{\infty}},\left\|Q_{ \pm}\right\|_{L_{\infty}},\left\|Q_{ \pm}^{-1}\right\|_{L_{\infty}}$, and the parameters of the lattice $\Gamma$.

We see that the third term of the corrector appeared in (12.8 \pm ).

12.2. The operator $\widehat{\mathcal{B}}_{\times}$. Now we consider the matrix operator composed of the blocks $\widehat{\mathcal{B}}_{-}\left(\omega_{-}^{2}\right)$ and $\widehat{\mathcal{B}}_{+}\left(\omega_{+}^{2}\right)$. Let $d=2$, let $m=n=2$, and let $\omega_{ \pm}(\mathbf{x})$ be two $\Gamma$-periodic functions subject to conditions (12.1 \pm ). We put

$$
h_{\times}=\operatorname{diag}\left\{\omega_{+}, \omega_{-}\right\}, \quad g_{\times}=h_{\times}^{2}=\operatorname{diag}\left\{\omega_{+}^{2}, \omega_{-}^{2}\right\} .
$$

In $\mathfrak{G}=L_{2}\left(\mathbb{R}^{2} ; \mathbb{C}^{2}\right)$, we consider the operators

$$
b_{\times}(\mathbf{D})=\left(\begin{array}{cc}
0 & \partial_{-} \\
\partial_{+} & 0
\end{array}\right), \quad \widehat{\mathcal{B}}_{\times}=b_{\times}(\mathbf{D}) g_{\times} b_{\times}(\mathbf{D}) .
$$

Then

$$
\widehat{\mathcal{B}}_{\times}=\operatorname{diag}\left\{\partial_{-} \omega_{-}^{2} \partial_{+}, \partial_{+} \omega_{+}^{2} \partial_{-}\right\}=\operatorname{diag}\left\{\widehat{\mathcal{B}}_{-}\left(\omega_{-}^{2}\right), \widehat{\mathcal{B}}_{+}\left(\omega_{+}^{2}\right)\right\} .
$$

The effective matrix for the operator (12.11) has the form

$$
g_{\times}^{0}=\underline{g_{\times}}=\operatorname{diag}\left\{g_{+}^{0}, g_{-}^{0}\right\}, \quad g_{ \pm}^{0}=\underline{\left(\omega_{ \pm}^{2}\right)},
$$

and the effective operator is given by

$$
\widehat{\mathcal{B}}_{\times}^{0}=b_{\times}(\mathbf{D}) g_{\times}^{0} b_{\times}(\mathbf{D})=\operatorname{diag}\left\{\widehat{\mathcal{B}}_{-}^{0}, \widehat{\mathcal{B}}_{+}^{0}\right\}, \quad \widehat{\mathcal{B}}_{ \pm}^{0}=g_{ \pm}^{0}(-\Delta) .
$$

Let $\mathbf{v}_{j} \in \widetilde{H}^{1}\left(\Omega ; \mathbb{C}^{2}\right), j=1,2$, be the solution of the equation

$$
b_{\times}(\mathbf{D}) g_{\times}\left(b_{\times}(\mathbf{D}) \mathbf{v}_{j}+\mathbf{e}_{j}\right)=0, \quad \int_{\Omega} \mathbf{v}_{j}(\mathbf{x}) d \mathbf{x}=0 .
$$


Then

$$
\mathbf{v}_{1}=\left(\begin{array}{c}
0 \\
v_{+}
\end{array}\right), \quad \mathbf{v}_{2}=\left(\begin{array}{c}
v_{-} \\
0
\end{array}\right)
$$

where $v_{ \pm}$is the solution of problem $(12.4 \pm)$. The role of the matrix $\Lambda(\mathbf{x})$ for the operator $\widehat{\mathcal{B}}_{\times}$is played by the matrix

$$
\Lambda_{\times}(\mathbf{x})=\left(\begin{array}{cc}
0 & v_{-}(\mathbf{x}) \\
v_{+}(\mathbf{x}) & 0
\end{array}\right)
$$

Consider the operator

$$
\widehat{\mathcal{B}}_{\times, \varepsilon}=b_{\times}(\mathbf{D}) g_{\times}^{\varepsilon} b_{\times}(\mathbf{D})=\operatorname{diag}\left\{\widehat{\mathcal{B}}_{-, \varepsilon}, \widehat{\mathcal{B}}_{+, \varepsilon}\right\} .
$$

Proposition 12.3. Let $\omega_{ \pm}$be two $\Gamma$-periodic functions satisfying $(12.1 \pm)$. Let $\widehat{\mathcal{B}}_{\times, \varepsilon}$ be the operator defined by (12.9), (12.10), and (12.15), and let $\widehat{\mathcal{B}}_{\times}^{0}$ be the effective operator defined by (12.12) and (12.13). Let $v_{ \pm} \in \widetilde{H}^{1}(\Omega)$ be the solution of problem $(12.5 \pm)$, and let $\Lambda_{\times}(\mathbf{x})$ be the matrix (12.14). Then for $\varepsilon>0$ we have

$$
\left(\widehat{\mathcal{B}}_{\times, \varepsilon}+I\right)^{-1}=\widehat{\mathcal{R}}_{\times}^{0}+\varepsilon \Lambda_{\times}^{\varepsilon} b_{\times}(\mathbf{D}) \widehat{\mathcal{R}}_{\times}^{0}+\varepsilon \widehat{\mathcal{R}}_{\times}^{0} b_{\times}(\mathbf{D})\left(\Lambda_{\times}^{\varepsilon}\right)^{*}+\varepsilon^{2} \widehat{\mathcal{I}}_{\times}(\varepsilon),
$$

where $\widehat{\mathcal{R}}_{\times}^{0}=\left(\widehat{\mathcal{B}}_{\times}^{0}+I\right)^{-1}=\operatorname{diag}\left\{\widehat{\mathcal{R}}_{-}^{0}, \widehat{\mathcal{R}}_{+}^{0}\right\}, \widehat{\mathcal{R}}_{ \pm}^{0}=\left(-g_{ \pm}^{0} \Delta+I\right)^{-1}$, and

$$
\left\|\widehat{\mathcal{I}}_{\times}(\varepsilon)\right\|_{\mathfrak{G} \rightarrow \mathfrak{G}} \leq \widehat{\mathcal{C}}_{\times} .
$$

The constant $\widehat{\mathcal{C}}_{\times}$depends only on $\left\|\omega_{ \pm}\right\|_{L_{\infty}},\left\|\omega_{ \pm}^{-1}\right\|_{L_{\infty}}$, and the parameters of the lattice $\Gamma$.

For the proof, it suffices to refer to Proposition 8.4, but the claim also follows from Proposition 12.1. Indeed, since all operators in (12.16) are diagonal, (12.16) can be written as

$$
\begin{aligned}
&\left(\widehat{\mathcal{B}}_{\times, \varepsilon}+I\right)^{-1}= \operatorname{diag}\left\{\left(\widehat{\mathcal{B}}_{-, \varepsilon}+I\right)^{-1},\left(\widehat{\mathcal{B}}_{+, \varepsilon}+I\right)^{-1}\right\} \\
&=\operatorname{diag}\left\{\widehat{\mathcal{R}}_{-}^{0}+\varepsilon v_{-}^{\varepsilon} \partial_{+} \widehat{\mathcal{R}}_{-}^{0}+\varepsilon \widehat{\mathcal{R}}_{-}^{0} \partial_{-}\left(v_{-}^{\varepsilon}\right)^{*}+\varepsilon^{2} \widehat{\mathcal{I}}_{-}(\varepsilon),\right. \\
&\left.\widehat{\mathcal{R}}_{+}^{0}+\varepsilon v_{+}^{\varepsilon} \partial_{-} \widehat{\mathcal{R}}_{+}^{0}+\varepsilon \widehat{\mathcal{R}}_{+}^{0} \partial_{+}\left(v_{+}^{\varepsilon}\right)^{*}+\varepsilon^{2} \widehat{\mathcal{I}}_{+}(\varepsilon)\right\} .
\end{aligned}
$$

Now, let $Q_{\times}(\mathbf{x})$ be a $\Gamma$-periodic $(2 \times 2)$-matrix (in general, with complex entries) such that

$$
Q_{\times}(\mathbf{x})>0, \quad Q_{\times}, Q_{\times}^{-1} \in L_{\infty} .
$$

We consider the approximation of the generalized resolvent $\left(\widehat{\mathcal{B}}_{\times, \varepsilon}+Q_{\times}^{\varepsilon}\right)^{-1}$. Now it is impossible to use splitting into blocks, unless the matrix $Q_{\times}$is diagonal. However, Proposition 9.5 implies the following statement.

Proposition 12.4. Under the assumptions of Proposition 12.3 , let $Q_{\times}(\mathbf{x})$ be a $\Gamma$-periodic $(2 \times 2)$-matrix-valued function satisfying (12.17). Then for $\varepsilon>0$ we have

$$
\begin{aligned}
\left(\widehat{\mathcal{B}}_{\times, \varepsilon}+Q_{\times}^{\varepsilon}\right)^{-1}= & \widehat{\mathcal{R}}_{\times, Q_{\times}}^{0}+\varepsilon \Lambda_{\times}^{\varepsilon} b_{\times}(\mathbf{D}) \widehat{\mathcal{R}}_{\times, Q_{\times}}^{0}+\varepsilon \widehat{\mathcal{R}}_{\times, Q_{\times}}^{0} b_{\times}(\mathbf{D})\left(\Lambda_{\times}^{\varepsilon}\right)^{*} \\
& -\varepsilon \widehat{\mathcal{R}}_{\times, Q_{\times}}^{0}\left(b_{\times}(\mathbf{D})\left(\overline{Q_{\times} \Lambda_{\times}}\right)^{*}+\left(\overline{Q_{\times} \Lambda_{\times}}\right) b_{\times}(\mathbf{D})\right) \widehat{\mathcal{R}}_{\times, Q_{\times}}^{0}+\varepsilon^{2} \widetilde{\mathcal{I}}_{\times}(\varepsilon),
\end{aligned}
$$

where $\widehat{\mathcal{R}}_{\times, Q_{\times}}^{0}=\left(\widehat{\mathcal{B}}_{\times}^{0}+\overline{Q_{\times}}\right)^{-1}$, and

$$
\left\|\widetilde{\mathcal{I}}_{\times}(\varepsilon)\right\|_{\mathfrak{G} \rightarrow \mathfrak{G}} \leq \widetilde{\mathcal{C}}_{\times}
$$

The constant $\widetilde{\mathcal{C}}_{\times}$depends only on $\left\|\omega_{ \pm}\right\|_{L_{\infty}},\left\|\omega_{ \pm}^{-1}\right\|_{L_{\infty}},\left\|Q_{\times}\right\|_{L_{\infty}},\left\|Q_{\times}^{-1}\right\|_{L_{\infty}}$, and the parameters of the lattice $\Gamma$. 
12.3. Definition and factorization of the Pauli operator. Let the magnetic potential $\mathbf{A}=\left\{A_{1}, A_{2}\right\}$ be a $\Gamma$-periodic real vector-valued function in $\mathbb{R}^{2}$ such that

$$
\mathbf{A} \in L_{p}\left(\Omega ; \mathbb{C}^{2}\right), \quad p>2 .
$$

We recall the standard notation for the Pauli matrices:

$$
\sigma_{1}=\left(\begin{array}{ll}
0 & 1 \\
1 & 0
\end{array}\right), \quad \sigma_{2}=\left(\begin{array}{cc}
0 & -i \\
i & 0
\end{array}\right), \quad \sigma_{3}=\left(\begin{array}{cc}
1 & 0 \\
0 & -1
\end{array}\right) .
$$

In the space $\mathfrak{G}=L_{2}\left(\mathbb{R}^{2} ; \mathbb{C}^{2}\right)$, we consider the operator

$$
\mathcal{D}=\left(D_{1}-A_{1}\right) \sigma_{1}+\left(D_{2}-A_{2}\right) \sigma_{2}, \quad \operatorname{Dom} \mathcal{D}=\mathfrak{G}^{1}=H^{1}\left(\mathbb{R}^{2} ; \mathbb{C}^{2}\right) .
$$

By definition, the Pauli operator $\mathcal{P}$ is the square of the operator $\mathcal{D}$ :

$$
\mathcal{P}=\mathcal{D}^{2}=\left(\begin{array}{cc}
P_{-} & 0 \\
0 & P_{+}
\end{array}\right) \text {. }
$$

The precise definition of the operator $\mathcal{P}$ is given in terms of the quadratic form $\|\mathcal{D} \mathbf{u}\|_{\mathfrak{G}}^{2}$, $\mathbf{u} \in \operatorname{Dom} \mathcal{D}$, which is closed in $\mathfrak{G}$. If the potential $\mathbf{A}$ is sufficiently smooth, then the blocks $P_{ \pm}$of the operator (12.20) are of the form

$$
P_{ \pm}=(\mathbf{D}-\mathbf{A})^{2} \pm B, \quad B=\partial_{1} A_{2}-\partial_{2} A_{1} .
$$

The expression $B$ corresponds to the strength of the magnetic field.

We use the known factorization for the Pauli operator (see, e.g., [BSu1, BSu2]). By a gauge transformation, we can ensure the conditions

$$
\operatorname{div} \mathbf{A}=0, \quad \int_{\Omega} \mathbf{A}(\mathbf{x}) d \mathbf{x}=0,
$$

without violating (12.19). Under conditions (12.19) and (12.22), there exists a (unique) $\Gamma$-periodic real-valued function $\varphi$ such that

$$
\nabla \varphi=\left\{A_{2},-A_{1}\right\}, \quad \int_{\Omega} \varphi(\mathbf{x}) d \mathbf{x}=0 .
$$

It turns out that $\varphi \in \widetilde{W}_{p}^{1}(\Omega) \subset C^{\alpha}, \alpha=1-2 p^{-1}$.

We introduce the notation

$$
\omega_{ \pm}(\mathbf{x})=e^{ \pm \varphi(\mathbf{x})} .
$$

Then $\omega_{ \pm} \in \widetilde{W}_{p}^{1}(\Omega)$ and

$$
\omega_{+}(\mathbf{x}) \omega_{-}(\mathbf{x})=1, \quad \mathbf{x} \in \mathbb{R}^{2} .
$$

Consider the matrices $h_{\times}$and $g_{\times}$defined by (12.9). The operators $\mathcal{D}$ and $\mathcal{P}$ admit the following factorization:

$$
\begin{aligned}
& \mathcal{D}=h_{\times} b_{\times}(\mathbf{D}) h_{\times}, \\
& \mathcal{P}=h_{\times} b_{\times}(\mathbf{D}) g_{\times} b_{\times}(\mathbf{D}) h_{\times} .
\end{aligned}
$$

For the blocks $P_{ \pm}$of $\mathcal{P}$ we have

$$
P_{+}=\omega_{-} \partial_{+} \omega_{+}^{2} \partial_{-} \omega_{-}, \quad P_{-}=\omega_{+} \partial_{-} \omega_{-}^{2} \partial_{+} \omega_{+} .
$$

It is convenient to view expressions (12.24)-(12.26) as the definitions of the operators $\mathcal{D}$, $\mathcal{P}$, and $P_{ \pm}$, assuming that the $\omega_{ \pm}$are arbitrary $\Gamma$-periodic functions satisfying $(12.1 \pm)$ and (12.23). More precisely, the operator $\mathcal{D}$ is given by (12.24) on the domain

$$
\operatorname{Dom} \mathcal{D}=\left\{\mathbf{u} \in \mathfrak{G}: h_{\times} \mathbf{u} \in \mathfrak{G}^{1}\right\} .
$$

The operator $\mathcal{P}$ is defined in terms of the quadratic form $\|\mathcal{D} \mathbf{u}\|_{\mathfrak{G}}^{2}, \mathbf{u} \in \operatorname{Dom} \mathcal{D}$. The blocks $P_{ \pm}$are defined via the quadratic forms

$$
\left\|\omega_{ \pm} \partial_{\mp} \omega_{\mp} u\right\|_{L_{2}\left(\mathbb{R}^{2}\right)}^{2}, \quad \omega_{\mp} u \in H^{1}\left(\mathbb{R}^{2}\right) .
$$


Observe that the operators $P_{+}$and $P_{-}$are unitarily equivalent. Moreover, for any quasimomentum $\mathbf{k}$, the operators $P_{+}(\mathbf{k})$ and $P_{-}(\mathbf{k})$ occurring in the direct integral decomposition for $P_{ \pm}$are unitarily equivalent.

12.4. The operators $P_{ \pm}$. The operators $P_{ \pm}$fall into the general framework. Now $d=2$ and $m=n=1$. From (12.26) it is clear that the operator $P_{ \pm}$has the form $\mathcal{A}(g, f)$ with $b(\mathbf{D})=\partial_{\mp}, g=\omega_{ \pm}^{2}, f=\omega_{\mp}$. The role of the corresponding operator $\widehat{\mathcal{A}}(g)$ is played by the operator $\widehat{\mathcal{B}}_{ \pm}($see $(12.2))$. Let $\lambda_{ \pm}(t, \boldsymbol{\theta})$ be the analytic (with respect to $t=|\mathbf{k}|$ ) eigenvalue of the operator $P_{ \pm}(\mathbf{k})$ (see Subsection 3.4), and let

$$
\lambda_{ \pm}(t, \boldsymbol{\theta})=\gamma_{ \pm}(\boldsymbol{\theta}) t^{2}+\mu_{ \pm}(\boldsymbol{\theta}) t^{3}+\cdots
$$

be the corresponding expansion. Since $P_{+}(\mathbf{k})$ and $P_{-}(\mathbf{k})$ are unitarily equivalent, we have $\lambda_{+}(t, \boldsymbol{\theta})=\lambda_{-}(t, \boldsymbol{\theta})$, whence

$$
\gamma_{+}(\boldsymbol{\theta})=\gamma_{-}(\boldsymbol{\theta}), \quad \mu_{+}(\boldsymbol{\theta})=\mu_{-}(\boldsymbol{\theta}) .
$$

Moreover, in [BSu1, BSu2] it was shown that, in fact, the numbers $\gamma_{ \pm}(\boldsymbol{\theta})$ do not depend on $\theta$ and are given by

$$
\gamma_{+}=\gamma_{-}=\left(\overline{\omega_{+}^{2}}\right)^{-1}\left(\overline{\omega_{-}^{2}}\right)^{-1}=: \gamma .
$$

By (12.23), we have $\underline{\omega_{+}^{2}}=\left(\overline{\omega_{-}^{2}}\right)^{-1}$ and $\underline{\omega_{-}^{2}}=\left(\overline{\omega_{+}^{2}}\right)^{-1}$.

We consider the operators

$$
P_{ \pm, \varepsilon}=\omega_{\mp}^{\varepsilon} \partial_{ \pm}\left(\omega_{ \pm}^{\varepsilon}\right)^{2} \partial_{\mp} \omega_{\mp}^{\varepsilon} .
$$

If the magnetic potential is sufficiently smooth, so that (12.21) makes sense, then

$$
P_{ \pm, \varepsilon}=\left(\mathbf{D}-\varepsilon^{-1} \mathbf{A}^{\varepsilon}\right)^{2} \pm \varepsilon^{-2} B^{\varepsilon} .
$$

By Proposition 9.5, approximation for $\left(P_{ \pm, \varepsilon}+I\right)^{-1}$ can be deduced directly from Proposition 12.2. We have $Q_{ \pm}=\omega_{ \pm}^{2}$, and, in the notation of Proposition 12.2,

$$
\widehat{\mathcal{R}}_{ \pm, Q_{ \pm}}^{0}=\left(-\underline{\omega_{ \pm}^{2}} \Delta+\overline{\omega_{ \pm}^{2}}\right)^{-1}=\left(\overline{\omega_{ \pm}^{2}}\right)^{-1}(-\gamma \Delta+I)^{-1}=: \widehat{\Re}_{ \pm},
$$

where $\gamma$ is the constant (12.28). Now $v_{ \pm} \in \widetilde{H}^{1}(\Omega)$ is the solution of the problem

$$
\partial_{\mp} v_{ \pm}=\underline{\omega_{ \pm}^{2}} \omega_{\mp}^{2}-1, \quad \int_{\Omega} v_{ \pm}(\mathbf{x}) d \mathbf{x}=0 .
$$

Multiplying (12.8土) by $\omega_{ \pm}^{\varepsilon}$ from both sides, we obtain the following statement.

Theorem 12.5( \pm ). Let $\omega_{+}$and $\omega_{-}$be two real-valued $\Gamma$-periodic functions on $\mathbb{R}^{2}$ such that $\omega_{+}, \omega_{-} \in L_{\infty}$ and $\omega_{+} \omega_{-}=1$. Suppose that the operator $P_{ \pm, \varepsilon}$ is defined by $(12.29 \pm)$, and the operator $\widehat{\Re}_{ \pm}$is defined by $(12.30 \pm)$. Let $v_{ \pm} \in \widetilde{H}^{1}(\Omega)$ be the solution of problem $(12.31 \pm)$. Then for $\varepsilon>0$ we have

$$
\left(P_{ \pm, \varepsilon}+I\right)^{-1}=\omega_{ \pm}^{\varepsilon}\left(\widehat{\Re}_{ \pm}+\varepsilon K_{ \pm}(\varepsilon)\right) \omega_{ \pm}^{\varepsilon}+\varepsilon^{2} \mathcal{Y}_{ \pm}(\varepsilon),
$$

$$
K_{ \pm}(\varepsilon)=v_{ \pm}^{\varepsilon} \partial_{\mp} \widehat{\mathfrak{R}}_{ \pm}+\widehat{\mathfrak{R}}_{ \pm} \partial_{ \pm}\left(v_{ \pm}^{\varepsilon}\right)^{*}-\widehat{\mathfrak{R}}_{ \pm}\left(2 D_{1}\left(\operatorname{Re} \overline{\omega_{ \pm}^{2} v_{ \pm}}\right) \pm 2 D_{2}\left(\operatorname{Im} \overline{\omega_{ \pm}^{2} v_{ \pm}}\right)\right) \widehat{\mathfrak{R}}_{ \pm}
$$

and

$$
\left\|\mathcal{Y}_{ \pm}(\varepsilon)\right\|_{L_{2}\left(\mathbb{R}^{2}\right) \rightarrow L_{2}\left(\mathbb{R}^{2}\right)} \leq \mathcal{C}_{ \pm}
$$

The constant $\mathcal{C}_{ \pm}$depends only on $\left\|\omega_{+}\right\|_{L_{\infty}},\left\|\omega_{-}\right\|_{L_{\infty}}$, and the parameters of the lattice $\Gamma$. 
The presence of the third term of the corrector in (12.32 \pm ) is related to the nonzero coefficient $\mu(\boldsymbol{\theta})=\mu_{+}(\boldsymbol{\theta})=\mu_{-}(\boldsymbol{\theta})$ in the expansion (12.27). From an abstract point of view, this corresponds to the influence of the summand $N_{0}(\boldsymbol{\theta})$ (see (1.15)-(1.17)) in the operator $N(\boldsymbol{\theta})$ (for $n=1$ the summand $N_{*}(\boldsymbol{\theta})$ is always zero).

Using (5.4), (5.12), and the fact that $L^{( \pm)}(\boldsymbol{\theta})=0$ (see Proposition $4.5(2)$ ), we find the symbols $L_{Q_{+}}^{(+)}(\boldsymbol{\theta})$ and $L_{Q_{-}}^{(-)}(\boldsymbol{\theta})$ (which play the role of $L_{Q}(\boldsymbol{\theta})$ ) for the operators $P_{ \pm}$:

$$
L_{Q_{ \pm}}^{( \pm)}(\boldsymbol{\theta})=-2 \gamma\left(\theta_{1} \operatorname{Re}\left(\overline{\omega_{ \pm}^{2} v_{ \pm}}\right) \pm \theta_{2} \operatorname{Im}\left(\overline{\omega_{ \pm}^{2} v_{ \pm}}\right)\right)
$$

Then, in accordance with (5.13) and (1.47),

$$
\mu_{ \pm}(\boldsymbol{\theta})=-2 \gamma\left(\overline{\bar{\omega}_{ \pm}^{2}}\right)^{-1}\left(\theta_{1} \operatorname{Re}\left(\overline{\omega_{ \pm}^{2} v_{ \pm}}\right) \pm \theta_{2} \operatorname{Im}\left(\overline{\omega_{ \pm}^{2} v_{ \pm}}\right)\right)
$$

Though we already know that $\mu_{+}(\boldsymbol{\theta})=\mu_{-}(\boldsymbol{\theta})$, the deduction of this identity from $(12.33 \pm)$ is nontrivial. It is easy to give examples of operators $P_{ \pm}$for which $\mu(\boldsymbol{\theta})$ is not equal to zero.

12.5. The operator $\mathcal{P}$. The operator $\mathcal{P}$ has the form $\mathcal{A}(g, f)$ with $b(\mathbf{D})=b_{\times}(\mathbf{D})$, $g=g_{\times}, f=h_{\times}$. Here $m=n=2$. The role of the corresponding operator $\widehat{\mathcal{A}}(g)$ is played by the operator $\widehat{\mathcal{B}}_{\times}$defined by $(12.10)$. We consider the operator

$$
\mathcal{P}_{\varepsilon}=h_{\times}^{\varepsilon} b_{\times}(\mathbf{D}) g_{\times}^{\varepsilon} b_{\times}(\mathbf{D}) h_{\times}^{\varepsilon} .
$$

By Proposition 9.5, an approximation for $\left(\mathcal{P}_{\varepsilon}+I\right)^{-1}$ can be obtained directly by multiplying (12.18) by $\left(h_{\times}^{\varepsilon}\right)^{-1}$ from both sides; now $Q_{\times}=\operatorname{diag}\left\{\omega_{-}^{2}, \omega_{+}^{2}\right\}$. Thus, we arrive at the following theorem.

Theorem 12.6. Suppose $\omega_{ \pm}$are two $\Gamma$-periodic functions satisfying $(12.1 \pm)$ and (12.23). Let $\mathcal{P}_{\varepsilon}$ be the operator defined by (12.9), (12.10), and $(12.34)$, let $\widehat{\mathfrak{R}}_{\times}=\operatorname{diag}\left\{\widehat{\mathfrak{R}}_{-}, \widehat{\mathfrak{R}}_{+}\right\}$, where the operators $\widehat{\mathfrak{R}}_{ \pm}$are defined by $(12.30 \pm)$, let $v_{ \pm} \in \widetilde{H}^{1}(\Omega)$ be the solution of the problem $(12.31 \pm)$, and let $\Lambda_{\times}$be defined by (12.14). We put $Q_{\times}=\operatorname{diag}\left\{\omega_{-}^{2}, \omega_{+}^{2}\right\}$. Then for $\varepsilon>0$ we have

$$
\begin{aligned}
\left(\mathcal{P}_{\varepsilon}+I\right)^{-1}= & \left(h_{\times}^{\varepsilon}\right)^{-1}\left(\widehat{\mathfrak{R}}_{\times}+\varepsilon \Lambda_{\times}^{\varepsilon} b_{\times}(\mathbf{D}) \widehat{\Re}_{\times}+\varepsilon \widehat{\Re}_{\times} b_{\times}(\mathbf{D})\left(\Lambda_{\times}^{\varepsilon}\right)^{*}\right. \\
& \left.\quad-\varepsilon \widehat{\Re}_{\times}\left(b_{\times}(\mathbf{D})\left(\overline{Q_{\times} \Lambda_{\times}}\right)^{*}+\left(\overline{Q_{\times} \Lambda_{\times}}\right) b_{\times}(\mathbf{D})\right) \widehat{\Re}_{\times}\right)\left(h_{\times}^{\varepsilon}\right)^{-1} \\
+ & \varepsilon^{2} \mathcal{Y}_{\times}(\varepsilon),
\end{aligned}
$$

and

$$
\left\|\mathcal{Y}_{\times}(\varepsilon)\right\|_{\mathfrak{G} \rightarrow \mathfrak{G}} \leq \mathcal{C}_{\times}
$$

The constant $\mathcal{C}_{\times}$depends only on $\left\|\omega_{+}\right\|_{L_{\infty}},\left\|\omega_{-}\right\|_{L_{\infty}}$, and the parameters of the lattice $\Gamma$.

Note that all operators in (12.35) are diagonal:

$$
\begin{aligned}
\left(\mathcal{P}_{\varepsilon}+I\right)^{-1} & =\operatorname{diag}\left\{\left(P_{-, \varepsilon}+I\right)^{-1},\left(P_{+, \varepsilon}+I\right)^{-1}\right\} \\
& =\operatorname{diag}\left\{\omega_{-}^{\varepsilon}\left(\widehat{\mathfrak{R}}_{-}+\varepsilon K_{-}(\varepsilon)\right) \omega_{-}^{\varepsilon}+\varepsilon^{2} \mathcal{Y}_{-}(\varepsilon), \omega_{+}^{\varepsilon}\left(\widehat{\Re}_{+}+\varepsilon K_{+}(\varepsilon)\right) \omega_{+}^{\varepsilon}+\varepsilon^{2} \mathcal{Y}_{+}(\varepsilon)\right\} .
\end{aligned}
$$

Therefore, Theorem 12.6 can be deduced directly from Theorem $12.5( \pm)$.

In the remainder of this section, we consider the approximation of the generalized resolvent of the operator $\mathcal{P}$. We use Proposition 9.10.

Theorem 12.7. Under the assumptions of Theorem 12.6 , let $\mathfrak{Q}_{\times}(\mathbf{x})$ be a $\Gamma$-periodic $(2 \times 2)$-matrix-valued function satisfying $(12.17)$. We put

$$
\mathfrak{Q}_{*}^{\times}=h_{\times}^{-1} \mathfrak{Q}_{\times} h_{\times}^{-1}=\left(\begin{array}{cc}
\omega_{-}^{2} \mathfrak{Q}_{\times}^{(11)} & \mathfrak{Q}_{\times}^{(12)} \\
\mathfrak{Q}_{\times}^{(21)} & \omega_{+}^{2} \mathfrak{Q}_{\times}^{(22)}
\end{array}\right) .
$$


Let $\widehat{\mathfrak{R}}_{*}^{\times}=\left(\widehat{\mathcal{B}}_{\times}^{0}+\overline{\mathfrak{Q}_{*}^{\times}}\right)^{-1}$, where $\widehat{\mathcal{B}}_{\times}^{0}$ is the operator defined by (12.12) and (12.13). Then for $\varepsilon>0$ we have

$$
\begin{aligned}
\left(\mathcal{P}_{\varepsilon}+\mathfrak{Q}_{\times}^{\varepsilon}\right)^{-1}= & \left(h_{\times}^{\varepsilon}\right)^{-1}\left(\widehat{\mathfrak{R}}_{*}^{\times}+\varepsilon \Lambda_{\times}^{\varepsilon} b_{\times}(\mathbf{D}) \widehat{\mathfrak{R}}_{*}^{\times}+\varepsilon \widehat{\mathfrak{R}}_{*}^{\times} b_{\times}(\mathbf{D})\left(\Lambda_{\times}^{\varepsilon}\right)^{*}\right. \\
& \left.\quad-\varepsilon \widehat{\mathfrak{R}}_{*}^{\times}\left(b_{\times}(\mathbf{D})\left(\overline{Q_{*}^{\times} \Lambda_{\times}}\right)^{*}+\left(\overline{Q_{*}^{\times} \Lambda_{\times}}\right) b_{\times}(\mathbf{D})\right) \widehat{\mathfrak{R}}_{*}^{\times}\right)\left(h_{\times}^{\varepsilon}\right)^{-1} \\
+ & +\varepsilon^{2} \mathcal{Y}_{*}^{\times}(\varepsilon),
\end{aligned}
$$

where

$$
\left\|\mathcal{Y}_{*}^{\times}(\varepsilon)\right\|_{\mathfrak{G} \rightarrow \mathfrak{G}} \leq \mathcal{C}_{*}^{\times} .
$$

The constant $\mathcal{C}_{*}^{\times}$depends only on $\left\|\omega_{+}\right\|_{L_{\infty}},\left\|\omega_{-}\right\|_{L_{\infty}},\left\|\mathfrak{Q}_{\times}\right\|_{L_{\infty}},\left\|\mathfrak{Q}_{\times}^{-1}\right\|_{L_{\infty}}$, and the parameters of the lattice $\Gamma$.

In [BSu2], along with $\mathcal{P}$, the "Pauli operator with metric" $\mathcal{P}_{\mathfrak{g}}=\mathcal{D} \mathfrak{g} \mathcal{D}$ was considered. Propositions 9.5 and 9.10 are applicable to that operator. We shall not write the corresponding formulations here.

\section{$\S 13$. THE OPERATOR OF ELASTICITY THEORY}

13.1. Definition of the operator. In this section, we assume that $d \geq 2$. We write the operator of elasticity theory as in BSu2, $\S 5.2$ ]. Let $\zeta$ be an orthogonal second rank tensor in $\mathbb{R}^{d}$; in the standard orthonormal basis in $\mathbb{R}^{d}$, it can be represented by a matrix $\zeta=\left\{\zeta_{j l}\right\}_{j, l=1}^{d}$. We shall consider symmetric tensors $\zeta$, which will be identified with vectors $\zeta_{*} \in \mathbb{C}^{m}, 2 m=d(d+1)$ by the following rule: $\zeta_{*}$ is formed by all elements $\zeta_{j l}$ with $j \leq l$, and the pairs $(j, l)$ are ordered in some fixed way.

For a displacement vector $\mathbf{u} \in \mathfrak{G}^{1}=H^{1}\left(\mathbb{R}^{d} ; \mathbb{C}^{d}\right)$, we introduce the deformation tensor

$$
e(\mathbf{u})=\frac{1}{2}\left\{\frac{\partial u_{j}}{\partial x_{l}}+\frac{\partial u_{l}}{\partial x_{j}}\right\} .
$$

Let $e_{*}(\mathbf{u})$ be the vector that corresponds to the tensor $e(\mathbf{u})$ in accordance with the rule described above. The relation

$$
b(\mathbf{D}) \mathbf{u}=-i e_{*}(\mathbf{u})
$$

gives rise to a unique $(m \times d)$-matrix homogeneous differential operator $b(\mathbf{D})$. Its symbol $b(\xi)$ has real entries. For instance, with an appropriate ordering we have

$$
b(\xi)=\left(\begin{array}{cc}
\xi^{1} & 0 \\
\frac{1}{2} \xi^{2} & \frac{1}{2} \xi^{1} \\
0 & \xi^{2}
\end{array}\right), \quad d=2 .
$$

Let $\sigma(\mathbf{u})$ be the stress tensor, and let $\sigma_{*}(\mathbf{u})$ be the corresponding vector. The Hooke law about proportionality of stress and deformation is expressed by the relation

$$
\sigma_{*}(\mathbf{u}):=g(\mathbf{x}) e_{*}(\mathbf{u}),
$$

where $g(\mathbf{x})$ is an $(m \times m)$-matrix (which gives an "economical" description of the Hooke tensor). The matrix $g(\mathbf{x})$ characterizes the parameters of an (in general, anisotropic) elastic medium. We assume that the matrix-valued function $g(\mathbf{x})$ is $\Gamma$-periodic and that $g(\mathbf{x})>0$ and $g, g^{-1} \in L_{\infty}$.

The quadratic form

$$
w[\mathbf{u}, \mathbf{u}]=\frac{1}{2} \int_{\mathbb{R}^{d}}\left\langle\sigma_{*}(\mathbf{u}), e_{*}(\mathbf{u})\right\rangle_{\mathbb{C}^{m}} d \mathbf{x}=\frac{1}{2} \int_{\mathbb{R}^{d}}\langle g(\mathbf{x}) b(\mathbf{D}) \mathbf{u}, b(\mathbf{D}) \mathbf{u}\rangle_{\mathbb{C}^{m}} d \mathbf{x}, \quad \mathbf{u} \in \mathfrak{G}^{1},
$$


gives the energy of elastic deformations. The operator $\mathcal{W}$ of elasticity theory corresponds to this form. Thus,

$$
2 \mathcal{W}=b(\mathbf{D})^{*} g b(\mathbf{D})=\widehat{\mathcal{A}}(g) .
$$

Now $n=d$ and $m=d(d+1) / 2$.

In the case of an isotropic medium, the matrix $g(\mathbf{x})$ depends only on two functional Lamé parameters $\lambda(\mathbf{x})$ and $\mu(\mathbf{x})$. The parameter $\mu$ is the shear modulus. Often, another parameter $K(\mathbf{x})$ is introduced instead of $\lambda(\mathbf{x}) ; K(\mathbf{x})$ is called the modulus of volume compression. We shall also use yet another modulus $\beta(\mathbf{x})$. Here are the relations:

$$
K(\mathbf{x})=\lambda(\mathbf{x})+\frac{2 \mu(\mathbf{x})}{d}, \quad \beta(\mathbf{x})=\mu(\mathbf{x})+\frac{\lambda(\mathbf{x})}{2} .
$$

The modulus $\lambda(\mathbf{x})$ may be negative. In the isotropic case, the conditions that ensure the positive definiteness of the matrix $g(\mathbf{x})$ are $\mu(\mathbf{x}) \geq \mu_{0}>0, K(\mathbf{x}) \geq K_{0}>0$. For instance, in the isotropic case, the matrix $g$ for $d=2$ has the form

$$
g_{\mu, K}(\mathbf{x})=\left(\begin{array}{ccc}
K+\mu & 0 & K-\mu \\
0 & 4 \mu & 0 \\
K-\mu & 0 & K+\mu
\end{array}\right), \quad d=2 .
$$

All our general results for operators of the form $\widehat{\mathcal{A}}(g)$ can be applied to the operator $\mathcal{W}$. In particular, Theorems 8.1 and 9.1(1) are applicable, and for $d \leq 4$, Theorems 8.2 and 9.3(1) are applicable. There are no simplifications in the general formulations (even for the isotropic medium with variable coefficients $\lambda$ and $\mu$ ).

13.2. Example. It is easy to give an example of the operator $\widehat{\mathcal{A}}=2 \mathcal{W}$ such that the third term $K_{3}$ of the corrector for $\left(\widehat{\mathcal{A}}_{\varepsilon}+I\right)^{-1}$ is not equal to zero. Let $d=2$, and let $\Gamma=(2 \pi \mathbb{Z})^{2}$. We consider the isotropic case. Suppose that the modulus $K$ is constant and that $\mu$ depends only on $x_{1}$. We calculate the effective matrix $g^{0}$ and the germ $\widehat{S}(\boldsymbol{\theta})=b(\boldsymbol{\theta})^{*} g^{0} b(\boldsymbol{\theta})$ :

$$
\begin{aligned}
& g^{0}=\left(\begin{array}{ccc}
(\underline{K+\mu}) & 0 & 2 K-\underline{(\underline{K+\mu})} \\
0 & 4 \underline{\mu} & 0 \\
2 K-(\underline{K+\mu}) & 0 & \underline{(K+\mu)}
\end{array}\right), \\
& \widehat{S}(\boldsymbol{\theta})=\left(\begin{array}{cc}
(\underline{K+\mu}) \theta_{1}^{2}+\underline{\mu} \theta_{2}^{2} & (2 K-(\underline{K+\mu})+\underline{\mu}) \theta_{1} \theta_{2} \\
(2 K-(\underline{K+\mu})+\underline{\mu}) \theta_{1} \theta_{2} & \underline{\mu} \theta_{1}^{2} \underline{+(\underline{K}+\mu)} \theta_{2}^{2}
\end{array}\right) .
\end{aligned}
$$

It is easily seen that the eigenvalues $\widehat{\gamma}_{1}(\boldsymbol{\theta})$ and $\widehat{\gamma}_{2}(\boldsymbol{\theta})$ of the germ $\widehat{S}(\boldsymbol{\theta})$ are different for all $\boldsymbol{\theta} \in \mathbb{S}^{1}$. Hence, by Corollary 4.3, relations (4.12) and (4.13) are fulfilled. Next,

$$
b(\boldsymbol{\theta})^{*} L(\boldsymbol{\theta}) b(\boldsymbol{\theta})=K(\underline{K+\mu}) \alpha \theta_{2}\left(\begin{array}{cc}
0 & i \\
-i & 0
\end{array}\right) .
$$

Here $\alpha=\overline{(K+\mu)^{-1} \Phi}$, and $\Phi\left(x_{1}\right)$ is the $(2 \pi)$-periodic solution of the problem

$$
\frac{1}{2} \frac{d \Phi}{d x_{1}}=\underline{\mu}\left(\mu\left(x_{1}\right)\right)^{-1}-1, \quad \int_{0}^{2 \pi} \Phi\left(x_{1}\right) d x_{1}=0 .
$$

Thus, the operator $\widehat{N}(\boldsymbol{\theta})=\widehat{N}_{*}(\boldsymbol{\theta})=b(\boldsymbol{\theta})^{*} L(\boldsymbol{\theta}) b(\boldsymbol{\theta}) \widehat{P}$ (corresponding to the third term of the corrector) is nonzero (for all $\theta$ with $\theta_{2} \neq 0$ ), unless $\alpha=0$. Now, the third term $K_{3}$ of the corrector for $\left(\widehat{\mathcal{A}}_{\varepsilon}+I\right)^{-1}$ is given by

$$
K_{3}=-K(\underline{K+\mu}) \alpha\left(\widehat{\mathcal{A}}^{0}+I\right)^{-1}(-\Delta)\left(\begin{array}{cc}
0 & \partial_{2} \\
-\partial_{2} & 0
\end{array}\right)\left(\widehat{\mathcal{A}}^{0}+I\right)^{-1} .
$$

It is easy to give an example of the coefficient $\mu\left(x_{1}\right)$ such that $\alpha \neq 0$. In particular, we can take $\mu=\left(3+\cos x_{1}+\sin 2 x_{1}\right)^{-1}$. 
Thus, the nonzero third term of the corrector may appear in a natural way in the problem with real-valued coefficients due to its vector character.

13.3. The possibility of interpolation for $d=2$. In the paper $[\mathrm{PaS}$, approximation of the resolvent was obtained for the two-dimensional operator of elasticity theory in the $\left(L_{2}\left(\mathbb{R}^{2} ; \mathbb{C}^{2}\right) \rightarrow H^{1}\left(\mathbb{R}^{2} ; \mathbb{C}^{2}\right)\right)$-norm. In our notation, the corresponding estimate can be written as

$$
\left\|\left(\widehat{\mathcal{A}}_{\varepsilon}+I\right)^{-1}-\left(\widehat{\mathcal{A}}^{0}+I\right)^{-1}-\varepsilon K_{1}^{\circ}(\varepsilon)\right\|_{L_{2}\left(\mathbb{R}^{2} ; \mathbb{C}^{2}\right) \rightarrow H^{1}\left(\mathbb{R}^{2} ; \mathbb{C}^{2}\right)} \leq c \varepsilon,
$$

where $\widehat{\mathcal{A}}_{\varepsilon}=\widehat{\mathcal{A}}\left(g^{\varepsilon}\right)$ corresponds to the operator $(13.2), \widehat{\mathcal{A}}^{0}=\widehat{\mathcal{A}}\left(g^{0}\right)$ is the effective operator, and $K_{1}^{\circ}(\varepsilon)=\Lambda^{\varepsilon} b(\mathbf{D})\left(\widehat{\mathcal{A}}^{0}+I\right)^{-1}$ corresponds to the first term of the corrector (8.14).

On the other hand, applying Theorem 8.2 to the operator (13.2), we obtain

$$
\left\|\left(\widehat{\mathcal{A}}_{\varepsilon}+I\right)^{-1}-\left(\widehat{\mathcal{A}}^{0}+I\right)^{-1}-\varepsilon K^{\circ}(\varepsilon)\right\|_{L_{2}\left(\mathbb{R}^{2} ; \mathbb{C}^{2}\right) \rightarrow L_{2}\left(\mathbb{R}^{2} ; \mathbb{C}^{2}\right)} \leq C \varepsilon^{2},
$$

where $K^{\circ}(\varepsilon)$ is the corrector (8.14). The same arguments as in Subsection 10.2 lead to the following estimate:

$$
\left\|\left(\widehat{\mathcal{A}}_{\varepsilon}+I\right)^{-1}-\left(\widehat{\mathcal{A}}^{0}+I\right)^{-1}-\varepsilon K^{\circ}(\varepsilon)\right\|_{L_{2}\left(\mathbb{R}^{2} ; \mathbb{C}^{2}\right) \rightarrow H^{s}\left(\mathbb{R}^{2} ; \mathbb{C}^{2}\right)} \leq c_{s} \varepsilon^{2-s}, \quad 0<s<1 .
$$

Unlike (10.10), now the corrector $K^{\circ}(\varepsilon)$ contains all three terms. The fact that the third term must be included is confirmed by the example in Subsection 13.2.

13.4. The Hill body. In mechanics (see, e.g., [ZhKO]), the elastic isotropic medium with constant shear modulus $\mu(\mathbf{x})=\mu_{0}=$ const is called the Hill body. In this case, a simpler factorization for the operator $\mathcal{W}$ is possible (see [BSu2, Subsection 5.2.3]). Now the energy form (13.1) can be represented as

$$
w[\mathbf{u}, \mathbf{u}]=\mu_{0} \int_{\mathbb{R}^{d}}|r(\mathbf{u})|^{2} d \mathbf{x}+\int_{\mathbb{R}^{d}} \beta(\mathbf{x})|\operatorname{div} \mathbf{u}|^{2} d \mathbf{x}, \quad \mathbf{u} \in \mathfrak{G}^{1},
$$

where

$$
r(\mathbf{u})=\frac{1}{2}\left\{\frac{\partial u_{j}}{\partial x_{l}}-\frac{\partial u_{l}}{\partial x_{j}}\right\} .
$$

The form (13.3) admits a more economical description than in the general case:

$$
w[\mathbf{u}, \mathbf{u}]=\int_{\mathbb{R}^{d}}\left\langle g_{\wedge} b_{\wedge}(\mathbf{D}) \mathbf{u}, b_{\wedge}(\mathbf{D}) \mathbf{u}\right\rangle_{\mathbb{C}^{m}} d \mathbf{x}
$$

(cf. [BSu2, §5.2]). Here $m_{\wedge}=1+d(d-1) / 2$. The $\left(m_{\wedge} \times d\right)$-matrix $b_{\wedge}(\xi)$ can be described as follows. The first row of $b_{\wedge}(\xi)$ coincides with $\left(\xi_{1}, \xi_{2}, \ldots, \xi_{d}\right)$. The other rows correspond to (different) pairs of indices $(j, l), 1 \leq j<l \leq d$. The element standing in the $(j, l)$ th row and the $j$ th column is $\xi_{l}$, and the element in the $(j, l)$ th row and the $l$ th column is $\left(-\xi_{j}\right)$; all other elements of the $(j, l)$ th row are equal to zero. The order of the rows is irrelevant. Finally,

$$
g_{\wedge}(\mathbf{x})=\operatorname{diag}\left\{\beta(\mathbf{x}), \mu_{0} / 2, \mu_{0} / 2, \ldots, \mu_{0} / 2\right\} .
$$

Thus,

$$
\mathcal{W}=b_{\wedge}(\mathbf{D})^{*} g_{\wedge}(\mathbf{x}) b_{\wedge}(\mathbf{D}) .
$$

As was shown in [BSu2, Subsection 5.2.3], the effective matrix $g_{\wedge}^{0}$ coincides with $\underline{g_{\wedge}}$ :

$$
g_{\wedge}^{0}=\underline{g_{\wedge}}=\operatorname{diag}\left\{\underline{\beta}, \mu_{0} / 2, \mu_{0} / 2, \ldots, \mu_{0} / 2\right\} .
$$

We construct the solutions $\mathbf{v}_{j} \in \widetilde{H}^{1}\left(\Omega ; \mathbb{C}^{d}\right), j=1, \ldots, m_{\wedge}$, of the problem

$$
b_{\wedge}(\mathbf{D})^{*} g_{\wedge}(\mathbf{x})\left(b_{\wedge}(\mathbf{D}) \mathbf{v}_{j}+\mathbf{e}_{j}\right)=0, \quad \int_{\Omega} \mathbf{v}_{j}(\mathbf{x}) d \mathbf{x}=0 .
$$


It is easily seen that $\mathbf{v}_{j}=0$ for $j=2, \ldots, m_{\wedge}$, and $\mathbf{v}_{1}(\mathbf{x})$ is the periodic solution of the problem

$$
-i \operatorname{div} \mathbf{v}_{1}=\underline{\beta}(\beta(\mathbf{x}))^{-1}-1, \quad r\left(\mathbf{v}_{1}\right)=0, \quad \int_{\Omega} \mathbf{v}_{1}(\mathbf{x}) d \mathbf{x}=0 .
$$

Then $\mathbf{v}_{1}=i \nabla \varphi$, where $\varphi$ is the periodic solution of the equation $\Delta \varphi=\underline{\beta}(\beta(\mathbf{x}))^{-1}-1$. Now the role of the matrix $\Lambda(\mathbf{x})$ is played by the $\left(d \times m_{\wedge}\right)$-matrix $\Lambda_{\wedge}(\mathbf{x})$ such that its first column is $\mathbf{v}_{1}=i \nabla \varphi(\mathbf{x})$ and the other columns are zero. Then $\Lambda_{\wedge}(\mathbf{x}) b(\mathbf{D})=(\nabla \varphi(\mathbf{x}))$ div. So, the conditions of Proposition 8.4 are satisfied. As a result, we obtain the following statement.

Theorem 13.1. Let $\mu=\mu_{0}=$ const, let $\beta(\mathbf{x})$ be a positive $\Gamma$-periodic function in $\mathbb{R}^{d}$ such that $\beta, \beta^{-1} \in L_{\infty}$, and let $g_{\wedge}(\mathbf{x})$ be the matrix defined by (13.4). We put

$$
\mathcal{W}_{\varepsilon}=b_{\wedge}(\mathbf{D})^{*} g_{\wedge}^{\varepsilon} b_{\wedge}(\mathbf{D})
$$

and $\mathcal{W}^{0}=b_{\wedge}(\mathbf{D})^{*} g_{\wedge} b_{\wedge}(\mathbf{D})$. Let $\varphi \in \widetilde{H}^{1}(\Omega)$ be the solution of the equation $\Delta \varphi=$ $\underline{\beta}(\beta(\mathbf{x}))^{-1}-1$, and let $\mathbf{p}=\nabla \varphi$. Then for $\varepsilon>0$ we have

(13.6) $\left(\mathcal{W}_{\varepsilon}+I\right)^{-1}=\left(\mathcal{W}^{0}+I\right)^{-1}+\varepsilon \mathbf{p}^{\varepsilon} \operatorname{div}\left(\mathcal{W}^{0}+I\right)^{-1}-\varepsilon\left(\mathcal{W}^{0}+I\right)^{-1} \nabla\left(\mathbf{p}^{\varepsilon}\right)^{t}+\varepsilon^{2} \mathcal{I}_{\wedge}(\varepsilon)$

and

$$
\left\|\mathcal{I}_{\wedge}(\varepsilon)\right\|_{\mathfrak{G} \rightarrow \mathfrak{G}} \leq \mathcal{C}_{\wedge} .
$$

The constant $\mathcal{C}_{\wedge}$ depends on $\|\beta\|_{L_{\infty}},\left\|\beta^{-1}\right\|_{L_{\infty}}$, d, and the parameters of the lattice $\Gamma$.

As always, if $g^{0}=\underline{g}$, then the third term of the corrector is equal to zero.

In conclusion, we consider the generalized resolvent $\left(\mathcal{W}_{\varepsilon}+Q^{\varepsilon}\right)^{-1}$. We use Proposition 9.5 .

Theorem 13.2. Under the assumptions of Theorem 13.1, suppose that $Q(\mathbf{x})$ is a $\Gamma$ periodic $(d \times d)$-matrix-valued function such that $Q(\mathbf{x})>0$ and $Q, Q^{-1} \in L_{\infty}$. Then for $\varepsilon>0$ we have

$$
\begin{aligned}
\left(\mathcal{W}_{\varepsilon}+Q^{\varepsilon}\right)^{-1}= & \left(\mathcal{W}^{0}+\bar{Q}\right)^{-1}+\varepsilon \mathbf{p}^{\varepsilon} \operatorname{div}\left(\mathcal{W}^{0}+\bar{Q}\right)^{-1}-\varepsilon\left(\mathcal{W}^{0}+\bar{Q}\right)^{-1} \nabla\left(\mathbf{p}^{\varepsilon}\right)^{t} \\
& -\varepsilon\left(\mathcal{W}^{0}+\bar{Q}\right)^{-1}\left((\overline{Q \mathbf{p}}) \operatorname{div}-\nabla(\overline{Q \mathbf{p}})^{t}\right)\left(\mathcal{W}^{0}+\bar{Q}\right)^{-1}+\varepsilon^{2} \widetilde{\mathcal{I}}_{\wedge}(\varepsilon)
\end{aligned}
$$

and

$$
\left\|\widetilde{\mathcal{I}}_{\wedge}(\varepsilon)\right\|_{\mathfrak{G} \rightarrow \mathfrak{G}} \leq \widetilde{\mathcal{C}}_{\wedge} .
$$

The constant $\widetilde{\mathcal{C}}_{\wedge}$ depends on $\|\beta\|_{L_{\infty}},\left\|\beta^{-1}\right\|_{L_{\infty}},\|Q\|_{L_{\infty}},\left\|Q^{-1}\right\|_{L_{\infty}}$, d, and the parameters of the lattice $\Gamma$.

\section{§14. The Model operator of electrodynamics}

14.1. Definition of the operator. In [BSu2, Chapter 7], when studying the homogenization problem for the stationary periodic Maxwell system, we introduced an auxiliary second-order operator $\mathcal{L}$. This operator acts in the space $\mathfrak{G}=L_{2}\left(\mathbb{R}^{3} ; \mathbb{C}^{3}\right)$ and is given by the expression

$$
\mathcal{L}=\mathcal{L}(\eta, \nu)=\operatorname{curl}(\eta(\mathbf{x}))^{-1} \operatorname{curl}-\nabla \nu(\mathbf{x}) \operatorname{div} .
$$

Here the $(3 \times 3)$-matrix-valued function $\eta(\mathbf{x})$ with real entries and a real-valued function $\nu(\mathbf{x})$ are $\Gamma$-periodic and satisfy

$$
\begin{aligned}
& \eta(\mathbf{x})>0, \quad \eta, \eta^{-1} \in L_{\infty}, \\
& \nu(\mathbf{x})>0, \quad \nu, \nu^{-1} \in L_{\infty} .
\end{aligned}
$$

The precise definition of the operator $\mathcal{L}$ is given in terms of the closed quadratic form

$$
\int_{\mathbb{R}^{3}}\left(\left\langle\eta(\mathbf{x})^{-1} \operatorname{curl} \mathbf{u}, \operatorname{curl} \mathbf{u}\right\rangle+\nu(\mathbf{x})|\operatorname{div} \mathbf{u}|^{2}\right) d \mathbf{x}, \quad \mathbf{u} \in \mathfrak{G}^{1}=H^{1}\left(\mathbb{R}^{3} ; \mathbb{C}^{3}\right) .
$$


The operator $\mathcal{L}$ can be written as $\widehat{\mathcal{A}}(g)=b(\mathbf{D})^{*} g(\mathbf{x}) b(\mathbf{D})$ with $n=3, m=4$, and

$$
b(\mathbf{D})=\left(\begin{array}{l}
-i \text { curl } \\
-i \operatorname{div}
\end{array}\right), \quad g(\mathbf{x})=\left(\begin{array}{cc}
(\eta(\mathbf{x}))^{-1} & 0 \\
0 & \nu(\mathbf{x})
\end{array}\right) .
$$

The symbol $b(\xi)$ looks like this:

$$
b(\xi)=\left(\begin{array}{ccc}
0 & -\xi_{3} & \xi_{2} \\
\xi_{3} & 0 & -\xi_{1} \\
-\xi_{2} & \xi_{1} & 0 \\
\xi_{1} & \xi_{2} & \xi_{3}
\end{array}\right) .
$$

14.2. Commentary. In [BSu2, Chapter 7], the homogenization problem for the stationary periodic Maxwell system was studied in the case where one of the coefficients (for instance, the dielectric permittivity) is given by a periodic matrix $\eta$, and the other coefficient is equal to $\mathbf{1}$. This problem was reduced (partially) to the study of the operator $\mathcal{L}$. In essence, the scalar-valued function $\nu$ in (14.1) has no influence on the result, and for applications to the Maxwell system it suffices to take $\nu=1$. However, we shall not "return" to the Maxwell system. The reason is the following. When both coefficients are periodic, the study of the Maxwell system cannot be reduced to the study of an operator of the form $\mathcal{A}=f^{*} b(\mathbf{D})^{*} g b(\mathbf{D}) f$. At the same time, the abstract method of BSu1, BSu2, BSu5] is applicable in this case, though specific constructions become much more complicated. In the general case, the periodic Maxwell system was studied in the paper [Su2] (see also [Su1]), where the "corrector of order zero" was introduced even for the approximation of the resolvent with the remainder term of order $\varepsilon$. However, such approximations were not found for all physically interesting fields. The authors are going to devote a separate paper to the homogenization problem for the periodic Maxwell system.

Still, the operator $\mathcal{L}$ of the form (14.1) is of interest in itself, and it exemplifies some specific features of the application of our general method. Therefore, here we consider the homogenization problem with corrector term for this operator.

14.3. Approximation of the resolvent. The effective matrix $g^{0}$ has the form (see [BSu2, §7.2])

$$
g^{0}=\left(\begin{array}{cc}
\left(\eta^{0}\right)^{-1} & 0 \\
0 & \underline{\nu}
\end{array}\right)
$$

where $\eta^{0}$ is the effective matrix for the scalar elliptic operator $-\operatorname{div} \eta \nabla=\mathbf{D}^{*} \eta \mathbf{D}$. The effective operator $\mathcal{L}^{0}$ is given by

$$
\mathcal{L}^{0}=\mathcal{L}\left(\eta^{0}, \underline{\nu}\right)=\operatorname{curl}\left(\eta^{0}\right)^{-1} \operatorname{curl}-\nabla \underline{\nu} \operatorname{div} .
$$

We construct the solutions $\mathbf{v}_{j} \in \widetilde{H}^{1}\left(\Omega ; \mathbb{C}^{3}\right), j=1,2,3,4$, of the problem

$$
b(\mathbf{D})^{*} g(\mathbf{x})\left(b(\mathbf{D}) \mathbf{v}_{j}+\mathbf{e}_{j}\right)=0, \quad \int_{\Omega} \mathbf{v}_{j}(\mathbf{x}) d \mathbf{x}=0 .
$$

Here the $\mathbf{e}_{j}, j=1,2,3,4$, are the standard unit vectors in $\mathbb{C}^{4}$. Let $\widetilde{\mathbf{e}}_{j}, j=1,2,3$, be the standard unit vectors in $\mathbb{C}^{3}$. We put

$$
\mathbf{c}_{j}=\left(\eta^{0}\right)^{-1} \widetilde{\mathbf{e}}_{j}, \quad j=1,2,3 .
$$

Let $\widetilde{\Phi}_{j}(\mathbf{x}), j=1,2,3$, be the $\Gamma$-periodic solution of the problem

$$
\operatorname{div} \eta(\mathbf{x})\left(\nabla \widetilde{\Phi}_{j}(\mathbf{x})+\mathbf{c}_{j}\right)=0, \quad \int_{\Omega} \widetilde{\Phi}_{j}(\mathbf{x}) d \mathbf{x}=0 .
$$


As was shown in [BSu2, $\S 7.2]$, for $j=1,2,3$ the vector-valued function $\mathbf{v}_{j}$ is the solution of the problem

$$
\operatorname{curl} \mathbf{v}_{j}=i\left(\eta\left(\nabla \widetilde{\Phi}_{j}+\mathbf{c}_{j}\right)-\widetilde{\mathbf{e}}_{j}\right), \quad \operatorname{div} \mathbf{v}_{j}=0, \quad \int_{\Omega} \mathbf{v}_{j} d \mathbf{x}=0 .
$$

Then

$$
\mathbf{v}_{j}=i \operatorname{curl} \mathbf{q}_{j}, \quad j=1,2,3,
$$

where $\mathbf{q}_{j}$ is the periodic solution of the problem

$$
\Delta \mathbf{q}_{j}=\eta\left(\nabla \widetilde{\Phi}_{j}+\mathbf{c}_{j}\right)-\widetilde{\mathbf{e}}_{j}, \quad \int_{\Omega} \mathbf{q}_{j} d \mathbf{x}=0
$$

Next,

$$
\mathbf{v}_{4}=i \nabla \varphi
$$

where $\varphi$ is the periodic solution of the problem

$$
\Delta \varphi=\underline{\nu}(\nu(\mathbf{x}))^{-1}-1, \quad \int_{\Omega} \varphi d \mathbf{x}=0 .
$$

We note that the functions $\widetilde{\Phi}_{j}(\mathbf{x}), \varphi(\mathbf{x}), \mathbf{q}_{j}(\mathbf{x})$ are real-valued, and the $\mathbf{v}_{j}, j=1,2,3,4$, are pure imaginary. The matrix $\Lambda(\mathbf{x})$ is the $(3 \times 4)$-matrix with the columns $i$ curl $\mathbf{q}_{1}$, $i \operatorname{curl} \mathbf{q}_{2}, i \operatorname{curl} \mathbf{q}_{3}, i \nabla \varphi$. We denote by $\Psi(\mathbf{x})$ the $(3 \times 3)$-matrix with the columns curl $\mathbf{q}_{1}$, $\operatorname{curl} \mathbf{q}_{2}, \operatorname{curl} \mathbf{q}_{3}$. This matrix has real entries. We put $\mathbf{w}=\nabla \varphi$. Then

$$
\Lambda(\mathbf{x}) b(\mathbf{D})=\Psi(\mathbf{x}) \operatorname{curl}+\mathbf{w}(\mathbf{x}) \operatorname{div}, \quad b(\mathbf{D})^{*}(\Lambda(\mathbf{x}))^{*}=\operatorname{curl}(\Psi(\mathbf{x}))^{t}-\nabla(\mathbf{w}(\mathbf{x}))^{t} .
$$

Next, now the matrix $\widetilde{g}(\mathbf{x})$ (see $(3.5)$ ) with real entries has a block-diagonal structure: the upper left $(3 \times 3)$-block is the matrix with the columns $\nabla \widetilde{\Phi}_{j}(\mathbf{x})+\mathbf{c}_{j}, j=1,2,3$; the lower right element is equal to $\underline{\nu}$, and the other elements are equal to zero. Calculating $L(\boldsymbol{\theta})$ by $(4.8)$ and using (14.8), we see that

$$
\begin{aligned}
L_{j k}(\boldsymbol{\theta}) & =i\left(\rho_{j k}(\boldsymbol{\theta})-\rho_{k j}(\boldsymbol{\theta})\right), & j, k & =1,2,3, \\
L_{4 j}(\boldsymbol{\theta}) & =L_{j 4}(\boldsymbol{\theta})=0, & j & =1,2,3,4 .
\end{aligned}
$$

Here

$$
\rho_{j k}(\boldsymbol{\theta})=|\Omega|^{-1} \int_{\Omega} \widetilde{\Phi}_{j}(\mathbf{x})\left\langle\eta(\mathbf{x})\left(\nabla \widetilde{\Phi}_{k}(\mathbf{x})+\mathbf{c}_{k}\right), \boldsymbol{\theta}\right\rangle d \mathbf{x}
$$

Obviously,

$$
\begin{aligned}
\rho_{j k}(\boldsymbol{\theta}) & =\sum_{s=1}^{3} \alpha_{j k s} \theta_{s}, \\
\alpha_{j k s} & =|\Omega|^{-1} \int_{\Omega} \widetilde{\Phi}_{j}(\mathbf{x})\left\langle\eta(\mathbf{x})\left(\nabla \widetilde{\Phi}_{k}(\mathbf{x})+\mathbf{c}_{k}\right), \mathbf{e}_{s}\right\rangle d \mathbf{x} .
\end{aligned}
$$

Calculating the symbol $b(\boldsymbol{\theta})^{*} L(\boldsymbol{\theta}) b(\boldsymbol{\theta})$, we obtain

$$
b(\boldsymbol{\theta})^{*} L(\boldsymbol{\theta}) b(\boldsymbol{\theta})=\left(L_{12}(\boldsymbol{\theta}) \theta_{3}+L_{31}(\boldsymbol{\theta}) \theta_{2}+L_{23}(\boldsymbol{\theta}) \theta_{1}\right)\left(\begin{array}{ccc}
0 & \theta_{3} & -\theta_{2} \\
-\theta_{3} & 0 & \theta_{1} \\
\theta_{2} & -\theta_{1} & 0
\end{array}\right) .
$$

This means that

$$
b(\mathbf{D})^{*} L(\mathbf{D}) b(\mathbf{D})=\mathcal{E}(\mathbf{D}) \operatorname{curl},
$$

where $\mathcal{E}(\mathbf{D})$ is a scalar homogeneous second-order DO:

$$
\begin{aligned}
\mathcal{E}(\mathbf{D}) & =i\left(L_{12}(\mathbf{D}) D_{3}+L_{31}(\mathbf{D}) D_{2}+L_{23}(\mathbf{D}) D_{1}\right) \\
& =\sum_{s=1}^{3}\left(\left(\alpha_{12 s}-\alpha_{21 s}\right) \partial_{3} \partial_{s}+\left(\alpha_{31 s}-\alpha_{13 s}\right) \partial_{2} \partial_{s}+\left(\alpha_{23 s}-\alpha_{32 s}\right) \partial_{1} \partial_{s}\right) .
\end{aligned}
$$


Since $d=3$, Condition 6.10 is satisfied. Applying Theorem 8.2, we obtain the following statement.

Theorem 14.1. Suppose that the matrix-valued function $\eta(\mathbf{x})$ with real entries and the real-valued function $\nu(\mathbf{x})$ are $\Gamma$-periodic and satisfy (14.2) and (14.3). Let $\mathcal{L}(\eta, \nu)$ be the operator (14.1), let

$$
\mathcal{L}_{\varepsilon}=\mathcal{L}\left(\eta^{\varepsilon}, \nu^{\varepsilon}\right),
$$

and let $\mathcal{L}^{0}=\mathcal{L}\left(\eta^{0}, \underline{\nu}\right)$, where $\eta^{0}$ is the effective matrix for the operator $\mathbf{D}^{*} \eta \mathbf{D}$. We put $\widehat{\mathcal{R}}^{0}=\left(\mathcal{L}^{0}+I\right)^{-1}$. We denote by $\widetilde{\Phi}_{j} \in \widetilde{H}^{1}(\Omega), j=1,2,3$, the solution of problem (14.6), (14.7), by $\mathbf{q}_{j}, j=1,2,3$, the $\Gamma$-periodic solution of problem (14.8), and by $\varphi(\mathbf{x})$ the $\Gamma$ periodic solution of problem (14.9). Let $\Psi(\mathbf{x})$ be the $(3 \times 3)$-matrix with the columns curl $\mathbf{q}_{j}, j=1,2,3$, let $\mathbf{w}=\nabla \varphi$, and let $\mathcal{E}(\mathbf{D})$ be the operator defined by (14.12), (14.14). Then for $\varepsilon>0$ we have

$$
\begin{aligned}
\left(\mathcal{L}_{\varepsilon}+I\right)^{-1}= & \widehat{\mathcal{R}}^{0}+\varepsilon\left(\Psi^{\varepsilon} \operatorname{curl}+\mathbf{w}^{\varepsilon} \operatorname{div}\right) \widehat{\mathcal{R}}^{0}+\varepsilon \widehat{\mathcal{R}}^{0}\left(\operatorname{curl}\left(\Psi^{\varepsilon}\right)^{t}-\nabla\left(\mathbf{w}^{\varepsilon}\right)^{t}\right) \\
& -\varepsilon \widehat{\mathcal{R}}^{0} \mathcal{E}(\mathbf{D}) \operatorname{curl} \widehat{\mathcal{R}}^{0}+\varepsilon^{2} \widehat{\mathcal{I}}^{\prime \prime}(\varepsilon)
\end{aligned}
$$

and

$$
\left\|\widehat{\mathcal{I}}^{\prime \prime}(\varepsilon)\right\|_{\mathfrak{G} \rightarrow \mathfrak{G}} \leq \widehat{\mathcal{C}}_{1}^{\prime \prime}
$$

The constant $\widehat{\mathcal{C}}_{1}^{\prime \prime}$ depends only on $\|\eta\|_{L_{\infty}},\left\|\eta^{-1}\right\|_{L_{\infty}},\|\nu\|_{L_{\infty}},\left\|\nu^{-1}\right\|_{L_{\infty}}$, and the parameters of the lattice.

14.4. Approximation of the generalized resolvent. Now, suppose that, along with the operator (14.1), a $\Gamma$-periodic $(3 \times 3)$-matrix $Q(\mathbf{x})$ with real entries is given, and that

$$
Q(\mathbf{x})>0, \quad Q, Q^{-1} \in L_{\infty} .
$$

The behavior of the generalized resolvent $\left(\mathcal{L}_{\varepsilon}+Q^{\varepsilon}\right)^{-1}$ is described in Theorem 9.3(1). Using the representation (9.11), (9.12), we obtain the following statement.

Theorem 14.2. Under the assumptions of Theorem 14.1 , let $Q(\mathbf{x})$ be a $\Gamma$-periodic $(3 \times 3)$ matrix-valued function with real entries and satisfying (14.17). Then for $\varepsilon>0$ we have

$$
\begin{aligned}
\left(\mathcal{L}_{\varepsilon}+Q^{\varepsilon}\right)^{-1}= & \widehat{\mathcal{R}}_{Q}^{0}+\varepsilon\left(\Psi^{\varepsilon} \operatorname{curl}+\mathbf{w}^{\varepsilon} \operatorname{div}\right) \widehat{\mathcal{R}}_{Q}^{0}+\varepsilon \widehat{\mathcal{R}}_{Q}^{0}\left(\operatorname{curl}\left(\Psi^{\varepsilon}\right)^{t}-\nabla\left(\mathbf{w}^{\varepsilon}\right)^{t}\right) \\
& -\varepsilon \widehat{\mathcal{R}}_{Q}^{0}\left(\mathcal{E}(\mathbf{D}) \operatorname{curl}+(\overline{Q \Psi}) \operatorname{curl}+\operatorname{curl}(\overline{Q \Psi})^{t}+(\overline{Q \mathbf{w}}) \operatorname{div}-\nabla(\overline{Q \mathbf{w}})^{t}\right) \widehat{\mathcal{R}}_{Q}^{0} \\
& +\varepsilon^{2} \widetilde{\mathcal{I}}^{\prime \prime}(\varepsilon)
\end{aligned}
$$

Here $\widehat{\mathcal{R}}_{Q}^{0}=\left(\mathcal{L}^{0}+\bar{Q}\right)^{-1}$, and

$$
\left\|\widetilde{\mathcal{I}}^{\prime \prime}(\varepsilon)\right\|_{\mathfrak{G} \rightarrow \mathfrak{G}} \leq \widetilde{\mathcal{C}}_{1}^{\prime \prime} .
$$

The constant $\widetilde{\mathcal{C}_{1}^{\prime \prime}}$ depends only on $\|\eta\|_{L_{\infty}},\left\|\eta^{-1}\right\|_{L_{\infty}},\|\nu\|_{L_{\infty}},\left\|\nu^{-1}\right\|_{L_{\infty}},\|Q\|_{L_{\infty}},\left\|Q^{-1}\right\|_{L_{\infty}}$, and the parameters of the lattice.

We have seen that, in the case of the operator (14.1), the calculation of the objects necessary for the final representation is more laborious than in $\S \S 10-13$.

14.5. Particular cases. The case where the correctors in (14.15) and (14.18) are zero is distinguished by Theorems 8.3 and 9.2. Now, the condition $g^{0}=\bar{g}$ means that

$$
\nu=\mathrm{const}, \quad \eta^{0}=\underline{\eta}
$$

(see (14.4), (14.5)). 
Theorem 14.3. Suppose that the operator $\mathcal{L}(\eta, \nu)$ satisfies the conditions of Theorem 14.1 , and that $\nu=$ Const. Suppose that the effective matrix $\eta^{0}$ for the operator $\mathbf{D}^{*} \eta \mathbf{D}$ coincides with $\underline{\eta}$, i.e., the columns of the matrix $\eta^{-1}$ admit the representations (10.19). Let $\mathcal{L}_{\varepsilon}=\mathcal{L}\left(\eta^{\varepsilon}, \nu\right)$, let $\mathcal{L}^{0}=\mathcal{L}\left(\eta^{0}, \nu\right)$, and let $Q(\mathbf{x})$ be the same matrix as in Theorem 14.2. Then for $\varepsilon>0$ we have

$$
\begin{aligned}
\left\|\left(\mathcal{L}_{\varepsilon}+I\right)^{-1}-\left(\mathcal{L}^{0}+I\right)^{-1}\right\|_{\mathfrak{G} \rightarrow \mathfrak{G}} & \leq \widehat{\mathcal{C}}_{2} \varepsilon^{2}, \\
\left\|\left(\mathcal{L}_{\varepsilon}+Q^{\varepsilon}\right)^{-1}-\left(\mathcal{L}^{0}+\bar{Q}\right)^{-1}\right\|_{\mathfrak{G} \rightarrow \mathfrak{G}} & \leq \mathcal{C}_{2} \varepsilon^{2} .
\end{aligned}
$$

The constant $\widehat{\mathcal{C}_{2}}$ depends on $\|\eta\|_{L_{\infty}},\left\|\eta^{-1}\right\|_{L_{\infty}}, \nu$, and the parameters of the lattice, and $\mathcal{C}_{2}$ depends on the same parameters and also on $\|Q\|_{L_{\infty}},\left\|Q^{-1}\right\|_{L_{\infty}}$.

The case where the third term of the corrector in (14.15) is zero was described in Proposition 8.4. Now, the condition $g^{0}=\underline{g}$ means that $\eta^{0}=\bar{\eta}$ (see (14.4) and (14.5)), and the coefficient $\nu(\mathbf{x})$ is arbitrary. In this case, the expression for the corrector in (14.18) simplifies (see Proposition 9.5). As a result, we obtain the following statement.

Proposition 14.4. Under the assumptions of Theorem 14.1, suppose that $\eta^{0}=\bar{\eta}$, i.e., the columns of the matrix $\eta$ satisfy conditions of the form (10.17). Let $Q(\mathbf{x})$ be the same matrix as in Theorem 14.2 .

1) Then for $\varepsilon>0$ we have

$$
\left(\mathcal{L}_{\varepsilon}+I\right)^{-1}=\widehat{\mathcal{R}}^{0}+\varepsilon\left(\Psi^{\varepsilon} \operatorname{curl}+\mathbf{w}^{\varepsilon} \operatorname{div}\right) \widehat{\mathcal{R}}^{0}+\varepsilon \widehat{\mathcal{R}}^{0}\left(\operatorname{curl}\left(\Psi^{\varepsilon}\right)^{t}-\nabla\left(\mathbf{w}^{\varepsilon}\right)^{t}\right)+\varepsilon^{2} \widehat{\mathcal{I}}^{\times}(\varepsilon)
$$

and

$$
\left\|\widehat{\mathcal{I}}^{\times}(\varepsilon)\right\|_{\mathfrak{G} \rightarrow \mathfrak{G}} \leq \widehat{\mathcal{C}}_{1}^{\times} .
$$

The constant $\widehat{\mathcal{C}_{1}^{\times}}$depends only on $\|\eta\|_{L_{\infty}},\left\|\eta^{-1}\right\|_{L_{\infty}},\|\nu\|_{L_{\infty}},\left\|\nu^{-1}\right\|_{L_{\infty}}$, and the parameters of the lattice.

2) For the generalized resolvent $\left(\mathcal{L}_{\varepsilon}+Q^{\varepsilon}\right)^{-1}$, the representation (14.18) is valid with the term $\widehat{\mathcal{R}}_{Q}^{0} \mathcal{E}(\mathbf{D})$ curl $\widehat{\mathcal{R}}_{Q}^{0}$ equal to zero.

Note that, in the case where the shear modulus is constant (see (13.5)) and $d=3$, the operator (14.1) will coincide with the operator $\mathcal{W}$ of elasticity theory if we put $\eta^{-1}=\left(\mu_{0} / 2\right) \mathbf{1}$ and $\nu(\mathbf{x})=\beta(\mathbf{x})$. Accordingly, the representation (14.19) turns into (13.6), and (14.18) turns into (13.7). (We recall that $\Psi=0$ and $\mathcal{E}(\mathbf{D})=0$ if $\eta=$ const.)

14.6. Example. It is easy to give an example of an operator $\mathcal{L}$ such that the third term $K_{3}$ of the corrector for $\left(\mathcal{L}_{\varepsilon}+I\right)^{-1}$ is nonzero. Let $\Gamma=(2 \pi \mathbb{Z})^{3}$. Suppose that the matrix $\eta(\mathbf{x})$ is diagonal and depends only on $x_{3}$ :

$$
\eta(\mathbf{x})=\operatorname{diag}\left\{\eta_{1}\left(x_{3}\right), \eta_{2}\left(x_{3}\right), \eta_{3}\left(x_{3}\right)\right\} .
$$

Here the $\eta_{j}\left(x_{3}\right), j=1,2,3$, are $(2 \pi)$-periodic positive functions. It is easily seen that in this case we have

$$
\eta^{0}=\operatorname{diag}\left\{\bar{\eta}_{1}, \bar{\eta}_{2}, \underline{\eta}_{3}\right\}
$$

and the solutions $\widetilde{\Phi}_{j}, j=1,2,3$, of problem (14.7) satisfy $\widetilde{\Phi}_{1}=\widetilde{\Phi}_{2}=0$, and $\widetilde{\Phi}_{3}(\mathbf{x})=$ $\Phi\left(x_{3}\right)$ solves the equation

$$
\Phi^{\prime}\left(x_{3}\right)=\left(\eta_{3}\left(x_{3}\right)\right)^{-1}-\left(\underline{\eta}_{3}\right)^{-1}, \quad \int_{0}^{2 \pi} \Phi\left(x_{3}\right) d x_{3}=0 .
$$


We calculate the symbols (14.11):

$$
\begin{aligned}
& \rho_{1 k}(\boldsymbol{\theta})=\rho_{2 k}(\boldsymbol{\theta})=0, \quad k=1,2,3, \\
& \rho_{31}(\boldsymbol{\theta})=\left(\overline{\eta_{1}}\right)^{-1}\left(\overline{\eta_{1} \Phi}\right) \theta_{1}, \\
& \rho_{32}(\boldsymbol{\theta})=\left(\overline{\eta_{2}}\right)^{-1}\left(\overline{\eta_{2} \Phi}\right) \theta_{2}, \\
& \rho_{33}(\boldsymbol{\theta})=0 .
\end{aligned}
$$

Hence, by (14.10) and (14.13), we have

$$
b(\boldsymbol{\theta})^{*} L(\boldsymbol{\theta}) b(\boldsymbol{\theta})=i \varkappa \theta_{1} \theta_{2}\left(\begin{array}{ccc}
0 & \theta_{3} & -\theta_{2} \\
-\theta_{3} & 0 & \theta_{1} \\
\theta_{2} & -\theta_{1} & 0
\end{array}\right),
$$

where $\varkappa=\left(\overline{\eta_{1}}\right)^{-1}\left(\overline{\eta_{1} \Phi}\right)-\left(\overline{\eta_{2}}\right)^{-1}\left(\overline{\eta_{2} \Phi}\right)$. Then, $\mathcal{E}(\mathbf{D})=\varkappa \partial_{1} \partial_{2}$ by (14.14). Now the third term $K_{3}$ of the corrector in (14.15) takes the form

$$
K_{3}=-\varkappa \widehat{\mathcal{R}}^{0} \partial_{1} \partial_{2} \operatorname{curl} \widehat{\mathcal{R}}^{0} .
$$

It is easy to find an example in which $\varkappa \neq 0$. In particular, we can put

$$
\eta_{1}\left(x_{3}\right)=2+\sin x_{3}, \quad \eta_{2}\left(x_{3}\right)=2, \quad \eta_{3}\left(x_{3}\right)=\left(3+\cos x_{3}+\sin 2 x_{3}\right)^{-1} .
$$

Then $\varkappa=1 / 4$. Moreover, if we put $\nu=1$, then the eigenvalues $\widehat{\gamma}_{j}(\boldsymbol{\theta}), j=1,2,3$, of the germ $\widehat{S}(\boldsymbol{\theta})=b(\boldsymbol{\theta})^{*} g^{0} b(\boldsymbol{\theta})$ can be calculated easily:

$$
\widehat{\gamma}_{1}(\boldsymbol{\theta})=1, \quad \widehat{\gamma}_{2}(\boldsymbol{\theta})=2, \quad \widehat{\gamma}_{3}(\boldsymbol{\theta})=\frac{1}{3}\left(\theta_{1}^{2}+\theta_{2}^{2}\right)+2 \theta_{3}^{2} .
$$

The spectrum of the germ is simple for all $\boldsymbol{\theta} \in \mathbb{S}^{2}$ except for two circles $\theta_{3}= \pm(2 / 5)^{1 / 2}$ and two points $\theta_{3}= \pm 1$. Therefore, by Corollary 4.3, relations (4.12) and (4.13) are valid. In this case, the operator $\widehat{N}(\boldsymbol{\theta})=b(\boldsymbol{\theta})^{*} L(\boldsymbol{\theta}) b(\boldsymbol{\theta}) \widehat{P}$ (that generates the third term of the corrector) coincides with $\widehat{N}_{*}(\boldsymbol{\theta})$ (see $(1.15)-(1.17)$ ).

Thus, we have one more example in which for a problem with real-valued coefficients the nonzero third term of the corrector is related to the vector nature of the problem.

14.7. Splitting of the operator $\mathcal{L}$. Since the operator $\mathcal{L}$ is related to the Maxwell system, it is of interest to study the representation (14.15) restricted to the divergence free subspace

$$
J=\{\mathbf{u} \in \mathfrak{G}: \operatorname{div} \mathbf{u}=0\} .
$$

We consider the orthogonal Weyl decomposition

$$
\mathfrak{G}=L_{2}\left(\mathbb{R}^{3} ; \mathbb{C}^{3}\right)=J \oplus G,
$$

where

$$
G=\left\{\mathbf{u}=\nabla \phi: \phi \in H_{\mathrm{loc}}^{1}\left(\mathbb{R}^{3}\right), \nabla \phi \in \mathfrak{G}\right\} .
$$

Obviously, the decomposition (14.21) reduces the operator (14.1):

$$
\mathcal{L}=\mathcal{L}_{J} \oplus \mathcal{L}_{G} .
$$

The operator $\mathcal{L}_{J}$ acts in the subspace $J$ and corresponds to the differential expression curl $\eta^{-1}$ curl, and the operator $\mathcal{L}_{G}$ acts in $G$ and is given by the expression $-\nabla \nu$ div. The operators $\mathcal{L}_{\varepsilon}$ and $\mathcal{L}^{0}$ are also reduced by the decomposition (14.21). We are interested in the operators $\mathcal{L}_{J}, \mathcal{L}_{J, \varepsilon}$, and $\mathcal{L}_{J}^{0}$. Since they are independent of the coefficient $\nu$, it suffices to consider the case where $\nu=1$.

Let $\mathcal{P}$ be the orthogonal projection of $\mathfrak{G}$ onto $J$. We restrict the representation (14.15) to $J$ and then multiply all terms by $\mathcal{P}$ from the left, obtaining

$(14.22)\left(\mathcal{L}_{J, \varepsilon}+I_{J}\right)^{-1}=\widehat{\mathcal{R}}_{J}^{0}+\varepsilon \mathcal{P} \Psi^{\varepsilon} \operatorname{curl} \widehat{\mathcal{R}}_{J}^{0}+\varepsilon \widehat{\mathcal{R}}_{J}^{0} \operatorname{curl}\left(\Psi^{\varepsilon}\right)^{t}-\varepsilon \widehat{\mathcal{R}}_{J}^{0} \mathcal{E}(\mathbf{D}) \operatorname{curl} \widehat{\mathcal{R}}_{J}^{0}+\varepsilon^{2} \mathcal{I}_{J}(\varepsilon)$, 
where $\widehat{\mathcal{R}}_{J}^{0}=\left(\mathcal{L}_{J}^{0}+I_{J}\right)^{-1}$ and all operators act in $J$. Here $\mathcal{I}_{J}(\varepsilon)=\left.\mathcal{P} \widehat{\mathcal{I}}^{\prime \prime}(\varepsilon)\right|_{J}$ and

$$
\left\|\mathcal{I}_{J}(\varepsilon)\right\|_{J \rightarrow J} \leq\left\|\mathcal{P} \widehat{\mathcal{I}}^{\prime \prime}(\varepsilon) \mathcal{P}\right\|_{\mathfrak{G} \rightarrow \mathfrak{G}} \leq \mathcal{C}_{J} .
$$

Thus, the following theorem is true.

Theorem 14.5. Suppose that the matrix-valued function $\eta(\mathbf{x})$ with real entries is $\Gamma$ periodic and satisfies conditions (14.2). Let $J$ be the subspace (14.20), and let $\mathcal{P}$ be the orthogonal projection of $\mathfrak{G}$ onto $J$. Let $\mathcal{L}_{J, \varepsilon}$ be the operator acting in $J$ and given by the expression $\operatorname{curl}\left(\eta^{\varepsilon}\right)^{-1}$ curl. Let $\eta^{0}$ be the effective matrix for the operator $\mathbf{D}^{*} \eta \mathbf{D}$, and let $\mathcal{L}_{J}^{0}$ be the operator acting in $J$ and given by the expression $\operatorname{curl}\left(\eta^{0}\right)^{-1}$ curl. We put $\widehat{\mathcal{R}}_{J}^{0}=\left(\mathcal{L}_{J}^{0}+I_{J}\right)^{-1}$. Let the matrix-valued function $\Psi(\mathbf{x})$ and the operator $\mathcal{E}(\mathbf{D})$ be the same as in the assumptions of Theorem 14.1. Then for $\varepsilon>0$ the representation (14.22) and estimate (14.23) are valid. The constant $\mathcal{C}_{J}$ depends on $\|\eta\|_{L_{\infty}},\left\|\eta^{-1}\right\|_{L_{\infty}}$, and the parameters of the lattice.

Similarly, Theorem 14.3 and Proposition 14.4 imply the following results.

Theorem 14.6. Under the assumptions of Theorem 14.5, suppose that $\eta^{0}=\eta$, i.e., the columns of the matrix $\eta(\mathbf{x})^{-1}$ admit representations of the form (10.19). Then

$$
\left\|\left(\mathcal{L}_{J, \varepsilon}+I_{J}\right)^{-1}-\left(\mathcal{L}_{J}^{0}+I_{J}\right)^{-1}\right\|_{\mathfrak{G} \rightarrow \mathfrak{G}} \leq \mathcal{C}_{J}^{\prime} \varepsilon^{2} .
$$

The constant $\mathcal{C}_{J}^{\prime}$ depends only on $\|\eta\|_{L_{\infty}},\left\|\eta^{-1}\right\|_{L_{\infty}}$, and the parameters of the lattice.

Proposition 14.7. Under the assumptions of Theorem 14.5, suppose that $\eta^{0}=\bar{\eta}$, i.e., the columns of the matrix $\eta(\mathbf{x})$ are divergence free. Then for $\varepsilon>0$ we have

$$
\left(\mathcal{L}_{J, \varepsilon}+I_{J}\right)^{-1}=\widehat{\mathcal{R}}_{J}^{0}+\varepsilon \mathcal{P} \Psi^{\varepsilon} \operatorname{curl} \widehat{\mathcal{R}}_{J}^{0}+\varepsilon \widehat{\mathcal{R}}_{J}^{0} \operatorname{curl}\left(\Psi^{\varepsilon}\right)^{t}+\varepsilon^{2} \widehat{\mathcal{I}}_{J}^{\times}(\varepsilon),
$$

where all operators act in $J$, and

$$
\left\|\widehat{\mathcal{I}}_{J}^{\times}(\varepsilon)\right\|_{\mathfrak{G} \rightarrow \mathfrak{G}} \leq \mathcal{C}_{J}^{\times} .
$$

The constant $\mathcal{C}_{J}^{\times}$depends only on $\|\eta\|_{L_{\infty}},\left\|\eta^{-1}\right\|_{L_{\infty}}$, and the parameters of the lattice.

Note that in (14.22) and (14.24) only one term involves the projection $\mathcal{P}$. We can ignore this projection without changing the remainder estimate. However, then the corrector will include a term lying off the divergence free subspace.

\section{§15. COMments AND ReFEREnCES}

In this section, we collect fairly diverse comments and references. Some comments can also be found in Subsections 8.5, 8.6, 9.5, and 14.2. When citing the results of other authors, we try to preserve the notation of the present paper. In references, we usually restrict ourselves to the statements where the remainder terms are estimated in some operator norm, and it is assumed that the corresponding matrix-valued function $g$ in $(0.1),(0.2)$ is subject to no restrictions besides the positive definiteness and the condition $g+g^{-1} \in L_{\infty}$. Also, we do not touch on estimates in perforated domains, etc.

15.1. In a bounded domain $\mathcal{O} \subset \mathbb{R}^{d}$, we consider an equation of the form

$$
b(\mathbf{D})^{*} g^{\varepsilon}(\mathbf{x}) b(\mathbf{D}) \mathbf{u}_{\varepsilon}=\mathbf{F} \in L_{2}(\mathcal{O})
$$

under some classical boundary condition on $\partial \mathcal{O}$. In order to describe a "correct" Ansatz for approximation of the solution $\mathbf{u}_{\varepsilon}$ with remainder estimate of a given order in $\varepsilon$, Bakhvalov [Ba1, Ba2, $\mathrm{BaPa}$ ] (see also [BeLP]) suggested a method of (formal) two-scale decompositions. This method is of heuristic value. Under some smoothness assumptions on $g, \mathbf{F}$, and $\partial \mathcal{O}$, it leads to estimates for the remainder terms of the corresponding approximations of $\mathbf{u}_{\varepsilon}$. Such estimates have been obtained for the scalar operator $\mathbf{D}^{*} g^{\varepsilon} \mathbf{D}$, 
for the operator of elasticity theory, etc. This method also applies to equations of the form (0.6) in $\mathbb{R}^{d}$. The corresponding approximations are not asymptotic in the precise sense: the coefficients of the powers of $\varepsilon$ themselves depend on $\varepsilon$, so that they are not determined uniquely.

Our constructions are based on the precise expansions (1.7) and (1.8) in powers of $t$. However, approximation of the resolvent (see (1.23) and (1.24)) already involves two parameters $t$ and $\varepsilon$. Accordingly, by this method the expression for the corrector $K^{\circ}(\varepsilon)$ in (8.14) is also not determined uniquely. But this does not concern the term $K_{3}$ (see (8.20)), which is independent of $\varepsilon$. For the details, see Subsections 8.5 and 8.6. The expansions (1.7) and (1.8) automatically lead to the expression for $K_{3}$, already in the analysis of the first order approximation. S. A. Nazarov explained to the authors that the expression (8.20) for $K_{3}$ can also be found by Bakhvalov's method. However, for this it is necessary to construct approximations of higher order. Though it seems strange, apparently, Bakhvalov's method does not distinguish the term $K_{2}^{\circ}(\varepsilon)$ in $(8.14)$.

15.2. As has already been mentioned, for the first time estimates of the type (0.10) were obtained in BSu1. In a more systematic and more general form, which includes the operators (0.4), the corresponding material is contained in $\mathrm{BSu} 2$. Later, estimates (0.10) for the scalar operator (with matrix $g$ with real entries) and for the operator of elasticity theory were obtained in the paper [Zh2, where another (nonspectral) method was applied. That method was based on using the first order (rather than only the zeroth order) approximation and on introducing an additional parameter (the shift by $\boldsymbol{\omega} \in \Omega$ ) in the equation. Then estimates of the form (0.10) were obtained by integration over $\boldsymbol{\omega}$. In this manner, it is difficult to control the constant $c$ in (0.10) explicitly.

15.3. A similar method was used in $\mathrm{Zh} 4, \mathrm{Pas}$ (see also $\mathrm{ZhPas}$ ) to obtain the estimates of order $\varepsilon$ in the $H^{1}$-norm. More precisely, estimates of the form (8.18) were considered, but with a truncated and smoothed corrector. (The "smoothing operators" are discussed below in Subsection 15.5.) The operator $\mathbf{D}^{*} g \mathbf{D}$ and the operator of elasticity theory were considered. Afterwards, for the operator $\mathbf{D}^{*} g \mathbf{D}$ and also for the operator of elasticity theory with $d=2$, the smoothing operators were eliminated.

In the same papers, equations in a bounded domain $\mathcal{O}$ with sufficiently smooth boundary under the Dirichlet or Neumann boundary conditions were treated. For the operator $\mathbf{D}^{*} g \mathbf{D}$, the estimates

$$
\begin{aligned}
\left\|u_{\varepsilon}-u_{0}\right\|_{L_{2}(\mathcal{O})} & \leq c \varepsilon^{1 / 2}\|F\|_{L_{2}(\mathcal{O})}, \\
\left\|u_{\varepsilon}-v_{\varepsilon}\right\|_{H^{1}(\mathcal{O})} & \leq c \varepsilon^{1 / 2}\|F\|_{L_{2}(\mathcal{O})}
\end{aligned}
$$

were obtained, where $v_{\varepsilon}$ is the first approximation corresponding to the truncated corrector. The constants in the estimates depend on $g$ and $\mathcal{O}$. Under the Dirichlet condition, in (15.1), $\varepsilon^{1 / 2}$ can be replaced by $\varepsilon^{d / 2(d-1)}$ for $d \geq 3$ and by $\varepsilon \log \left(\varepsilon^{-1}\right)$ for $d=2$.

For the operator of elasticity theory (under the Dirichlet or Neumann condition), analogs of estimates (15.1) and (15.2) are valid, with the first approximation $v_{\varepsilon}$ in (15.2) replaced by the smoothed first approximation $\widetilde{v}_{\varepsilon}$.

15.4. Estimates in the $H^{1}$-norm in bounded domains were studied also in [G1, G2]. The operator $\mathbf{D}^{*} g \mathbf{D}$ under the Dirichlet or Neumann condition was considered. The nonspectral "unfolding method" was used, partially similar to the method of Zh4, Pas, ZhPas. In particular, in G1, estimate (15.1) and an estimate of the form (15.2) (but with smoothing operators) were obtained. In G2, for the Dirichlet problem, the estimate

$$
\left\|u_{\varepsilon}-\widetilde{v}_{\varepsilon}\right\|_{H^{1}\left(\mathcal{O}^{\prime}\right)} \leq C \varepsilon\|F\|_{L_{2}(\mathcal{O})}
$$


was announced. Here $\widetilde{v}_{\varepsilon}$ is the smoothed first approximation, $\mathcal{O}^{\prime}$ is a strictly interior subdomain of $\mathcal{O}$, and $C=C\left(g, \mathcal{O}, \mathcal{O}^{\prime}\right)$.

15.5. Smoothing operators. In the present paper, the operator $\Pi_{\varepsilon}$ defined by (8.8) has been used systematically as a smoothing operator. For the first time, this operator appeared in the paper Su2] devoted to the Maxwell operator. In that paper, it was possible to eliminate the operator $\Pi_{\varepsilon}$. In general, this operator is natural for the spectral approach. In particular, it appeared also in [Zh3].

In the papers mentioned in Subsection 15.3, a "dual" smoothing operator arose. This operator is the Steklov averaging in the " $\mathrm{x}$ "-representation. In [Zh4], for the operator $\mathbf{D}^{*} g \mathbf{D}$ this smoothing operator was eliminated on the basis of Proposition 8.2 in [Su2], where the multiplier properties of solutions of the problem on the cell were treated. In G1, G2, the smoothing operator was close to the Steklov averaging, but was bulkier. The author of [G1, G2] has not succeeded in eliminating this smoothing operator even in the case of the operator $\mathbf{D}^{*} g \mathbf{D}$.

15.6. The periodic operator $\widehat{\mathcal{A}}_{\varepsilon}=\mathbf{D}^{*} g^{\varepsilon} \mathbf{D}$ in $L_{2}\left(\mathbb{R}^{d}\right)$ was also studied in the paper COrV]. First of all, an equation of the form (0.6) was dealt with, but after that the families $\left\{u_{\varepsilon}\right\}$ for which $\widehat{\mathcal{A}}_{\varepsilon} u_{\varepsilon}=F$ and $u_{\varepsilon}$ converges to $u^{0} \in H^{1}\left(\mathbb{R}^{d}\right)$ weakly in $H^{1}\left(\mathbb{R}^{d}\right)$ and strongly in $L_{2}\left(\mathbb{R}^{d}\right)$ were discussed. For such families the main Theorems 1.8 and 1.11 of $\mathrm{COrV}$ ] imply the estimates

$$
\begin{aligned}
\left\|u_{\varepsilon}-u^{0}\right\|_{L_{2}\left(\mathbb{R}^{d}\right)} & \leq c \varepsilon\|F\|_{L_{2}\left(\mathbb{R}^{d}\right)}, \\
\left\|u_{\varepsilon}-u^{0}-\varepsilon \sum_{j=1}^{d} \Phi_{j}^{\varepsilon} \partial_{j} u^{0}\right\|_{H^{1}\left(\mathbb{R}^{d}\right)} & \leq c \varepsilon\|F\|_{L_{2}\left(\mathbb{R}^{d}\right)},
\end{aligned}
$$

where $\widehat{\mathcal{A}}^{0} u^{0}=F$, and (in our notation) the $\Phi_{j}$ are the solutions of equations (10.3). Estimate (15.4) was obtained under the additional condition $\nabla \Phi_{j} \in L_{\infty}(\Omega)$. However, for us it remains unclear what is implied by estimates (15.3) and (15.4) for the solution of equation (0.6).

15.7. Applications of the spectral approach (the Bloch approach) to homogenization problems date back to the papers [Se, Zh1] (see also [ZhKO]). In those papers the scalar operator $\mathbf{D}^{*} g \mathbf{D}$ was studied, and in [Zh1] the corresponding parabolic equation was considered. The problem concerning remainder estimates in the operator norm was not touched on in these papers. This investigation was based substantially upon smoothness assumptions.

In the case of the scalar operator $\mathbf{D}^{*} g \mathbf{D}$, the use of the spectral approach is strongly simplified by the fact that the lowest eigenvalue and the corresponding eigenfunction for $\widehat{\mathcal{A}}(\mathbf{k})$ are analytic in $\mathbf{k}$ (and not only in $t=|\mathbf{k}|$ ). Moreover, the corresponding theorem about expansion with respect to the eigenfunctions of the continuous spectrum is sufficiently transparent. On this basis, in $\mathrm{BSu} 3$, (and before in $\mathrm{B}$ for $d=1$ ), an analog of the homogenization procedure for the operator $\mathbf{D}^{*} g \mathbf{D}$, related to the edge of some gap in the continuous spectrum, was considered, and remainder estimates of order $\varepsilon$ in the operator $L_{2}\left(\mathbb{R}^{d}\right)$-norm were obtained. Herewith, the usual homogenization procedure, which corresponds to the bottom of the spectrum, was not excluded. This is a simpler and more direct way to the proof of estimate $(0.10)$ for the operator $\mathbf{D}^{*} g \mathbf{D}$. However, using this method, it is more difficult to explicitly control the constant in the estimate. Similarly, estimate (0.10) for the operator $\mathbf{D}^{*} g \mathbf{D}$ was obtained in the Introduction to the paper [Zh3]. 
15.8. For the first time, a remainder estimate of the form $(0.13)$ (of order $\varepsilon^{2}$ ) for the operator (0.5) was pointed out in two particular cases in BSu4. We mean the scalar operator $\mathbf{D}^{*} g \mathbf{D}$, where the matrix $g$ has real entries, and the operator $(0.5)$ with $m=n$. In both cases the corrector $K(\varepsilon)$ does not involve the third term $K_{3}$. In [BSu4], it was also pointed out that in the general case the corrector $K(\varepsilon)$ in $(0.13)$ must involve the term $K_{3}$. Recently, an estimate of the form (0.13) for the operator $\mathbf{D}^{*} g \mathbf{D}$ was also obtained by the spectral method in the paper [Zh3], where the operator $\mathbf{D}^{*} g \mathbf{D}$ was considered in the spaces $L_{2}\left(\mathbb{R}^{d}, \mu_{\varepsilon}\right)$; here $\mu_{\varepsilon}(\cdot)=\varepsilon^{d} \mu\left(\varepsilon^{-1} \cdot\right)$ and $\mu$ is a periodic measure. Here we do not discuss such problems.

Estimate (0.13) for the general operators (0.5) is given in the present paper for the first time. The same concerns the remainder estimates with corrector term for operators of the form (0.4); see statement 2) in Theorem 9.1.

\section{REFERENCES}

[Ba1] N. S. Bakhvalov, The homogenized characteristics of the medium with periodic structure, Dokl. Akad. Nauk SSSR 218 (1974), no. 5, 1046-1048; English transl., Soviet Phys. Dokl. 19 (1974), no. 10, 650-651 (1975). MR0395435 (52:16232)

[Ba2] Homogenization of partial differential equations with rapidly oscillating coefficients, Dokl. Akad. Nauk SSSR 221 (1975), no. 3, 516-519; English transl., Soviet Math. Dokl. 16 (1975), no. 2, 351-355. MR0380075 (52:976)

[BaPa] N. S. Bakhvalov and G. P. Panasenko, Homogenization: Averaging of processes in periodic media, Mathematical Problems in the Mechanics of Composite Materials, "Nauka", Moscow, 1984, 352 pp.; English transl., Math. Appl. (Soviet Ser.), vol. 36, Kluwer Acad. Publishers Group, Dordrecht, 1989. MR.0797571 (86m:73049), MR.1112788 (92d:73002)

[BeLP] A. Bensoussan, J.-L. Lions, and G. Papanicolaou, Asymptotic analysis for periodic structures, Stud. Math. Appl., vol. 5, North-Holland Publishing Co., Amsterdam-New York, 1978, 700 pp. MR:0503330 (82h:35001)

[B] M. Sh. Birman, On homogenization procedure for periodic operators near the edge of an internal gap, Algebra i Analiz 15 (2003), no. 4, 61-71; English transl., St. Petersburg Math. J. 15 (2004), no. 4, 507-513. MR2068979

[BSu1] M. Birman and T. Suslina, Threshold effects near the lower edge of the spectrum for periodic differential operators of mathematical physics, Systems, Approximation, Singular Integral Operators, and Related Topics (Bordeaux, 2000), Oper. Theory Adv. Appl., vol. 129, Birkhäuser, Basel, 2001, pp. 71-107. MR1882692 (2003f:35220)

[BSu2] _ Second-order periodic differential operators. Threshold properties and homogenization, Algebra i Analiz 15 (2003), no. 5, 1-108; English transl., St. Petersburg Math. J. 15 (2004), no. 5, 639-714. MR2068790 (2005k:47097)

[BSu3] _ Homogenization of a multidimensional periodic elliptic operator near the edge of an internal gap, Boundary Value Problems of Mathematical Physics and Related Topics of Function Theory. 36, Zap. Nauchn. Sem. S.-Peterburg. Otdel. Mat. Inst. Steklov. (POMI) 318 (2004), 6074. (Russian) MR2120232(2005j:35006)

[BSu4] M. Sh. Birman (joint work with T. A. Suslina), Homogenization of periodic differential operators in $\mathbb{R}^{d}$ as a spectral threshold effect, Mathematisches Forschungsinstitut Oberwolfach, Report No. 53/2004, Spectral Analysis of Partial Differential Equations, November 28th - December 4th, 2004, pp. 2847-2849.

[BSu5] M. Sh. Birman and T. A. Suslina, Threshold approximations with corrector term for the resolvent of a factorized selfadjoint operator family, Algebra i Analiz 17 (2005), no. 5, 69-90; English transl. in St. Petersburg Math. J. 17 (2006), no. 5.

[COrV] C. Conca, R. Orive, and M. Vanninathan, Bloch approximation in homogenization and applications, SIAM J. Math. Anal. 33 (2002), no. 5, 1166-1198. MR1897707 (2003d:35015)

[G1] G. Griso, Error estimate and unfolding for periodic homogenization, Asymptot. Anal. 40 (2004), 269-286. MR2107633 (2006a:35015)

[G2] Interior error estimate for periodic homogenization, C. R. Math. Acad. Sci. Paris 340 (2005), 251-254. MR2123038(2005k:49040)

[Zh1] V. V. Zhikov, Spectral approach to asymptotic diffusion problems, Differentsial'nye Uravneniya 25 (1989), no. 1, 44-50; English transl., Differential Equations 25 (1989), no. 1, 33-39. MR.0986395 (90a:35107) 
[Zh2] - On operator estimates in homogenization theory, Dokl. Akad. Nauk 403 (2005), no. 3, 305-308. (Russian) MR2164541

[Zh3] - On the spectral method in homogenization theory, Trudy Mat. Inst. Steklova 250 (2005), 95-104; English transl., Proc. Steklov Inst. Math. 2005, no. 3 (250), 85-94. MR2200910

[Zh4] On some estimates of the homogenization theory, Dokl. Akad. Nauk (to appear).

[ZhKO] V. V. Zhikov, S. M. Kozlov, and O. A. Oleŭnik, Homogenization of differential operators, "Nauka", Moscow, 1993; English transl., Homogenization of differential operators and integral functionals, Springer-Verlag, Berlin, 1994. MR.1318242 (96h:35003a) MR1329546(96h:35003b)

[ZhPas] V. V. Zhikov and S. E. Pastukhova, On operator estimates for some problems in homogenization theory, Russ. J. Math. Phys. 12 (2005), no. 4, 515-524. (English) MR2201316

[Ka] T. Kato, Perturbation theory for linear operators, 2nd ed., Grundlehren Math. Wiss., vol. 132, Springer-Verlag, Berlin-New York, 1976. MR0407617 (53:11389)

[LaU] O. A. Ladyzhenskaya and N. N. Ural'tseva, Linear and quasilinear equations of elliptic type, 2nd ed., "Nauka", Moscow, 1973; English transl. of 1st ed., Acad. Press, New York-London, 1968. MR0509265 (58:23009) MR0244627 (39:5941)

[Ma] J. Marcinkiewicz, Sur les multiplicateurs des series de Fourier, Studia Math. 8 (1939), $78-91$.

[Mi] S. G. Mikhlin, Multidimensional singular integrals and integral equations, Fizmatgiz, Moscow, 1962; English transl., Pergamon Press, Oxford, 1965. MR0155165 (27:5105) MR0185399 $(32: 2866)$

[Pas] S. E. Pastukhova, On some estimates for homogenization problems of elasticity theory, Dokl. Akad. Nauk (to appear).

[Se] E. V. Sevost'yanova, Asymptotic expansion of the solution of a second-order elliptic equation with periodic rapidly oscillating coefficients, Mat. Sb. (N.S.) 115 (1981), no. 2, 204-222; English transl., Math. USSR-Sb. 43 (1982), no. 2, 181-198. MR0622145 (83d:35038)

[Su1] T. A. Suslina, On the homogenization of the periodic Maxwell system, Funktsional. Anal. i Prilozhen. 38 (2004), no. 3, 90-94; English transl., Funct. Anal. Appl. 38 (2004), no. 3, 234237. MR2095137 (2005g:35017)

[Su2] Homogenization of a stationary periodic Maxwell system, Algebra i Analiz 16 (2004), no. 5, 162-244; English transl., St. Petersburg Math. J. 16 (2005), no. 5, 863-922. MR2106671 (2005h:35019)

[Su3] , Homogenization of periodic parabolic systems, POMI Preprint no. 15/2004. (Russian)

[Su4] Homogenization of periodic parabolic systems, Funktsional. Anal. i Prilozhen. 38 (2004), no. 4, 86-90; English transl., Funct. Anal. Appl. 38 (2004), no. 4, 309-312. MR2117512 (2005j:35008)

[Sh1] R. G. Shterenberg, An example of a periodic magnetic Schrödinger operator with degenerate lower edge of the spectrum, Algebra i Analiz 16 (2004), no. 2, 177-185; English transl., St. Petersburg Math. J. 16 (2005), no. 2, 417-422. MR2068347 (2005d:35220)

[Sh2] - On the structure of the lower edge of the spectrum of the periodic magnetic Schrödinger operator with small magnetic potential, Algebra i Analiz 17 (2005), no. 5, 232-243; English transl. in St. Petersburg Math. J. 17 (2006), no. 5.

Department of Physics, St. Petersburg State University, Ulyanovskaya 1, Petrodvorets, St. Petersburg 198504, Russia

E-mail address: mbirman@list.ru

Department of Physics, St. Petersburg State University, Ulyanovskaya 1, Petrodvorets, St. Petersburg 198504, Russia

E-mail address: suslina@list.ru

Received 17/OCT/2005

Translated by T. A. SUSLINA 